\title{
V. Transformation und monarchische Prärogative: Der Niedergang des amerikanischen Reformismus, 1962-1963
}

\author{
V. 1. Das Scheitern des «Amini-Experiments» und die Re-Etablierung der Schah- \\ Autokratie
}

Noch im Oktober 1961 konnte man den Eindruck gewinnen, dass die amerikanische Entschlossenheit, das „Amini-Experiment“ in Iran zu einem erfolgreichen Ende zu führen, ungebrochen ist. Im Anschluss an die anglo-amerikanische Intervention zugunsten der Reformregierung schien tatsächlich ein neues, einigen Optimismus rechtfertigendes Verhältnis zwischen Ministerpräsident Amini und Mohammad Rezā entstanden zu sein. ${ }^{1}$ Chester Bowles stattete in seiner neuen Rolle als Sonderbeauftragter des Präsidenten für die Dritte Welt im Februar 1962 Teheran einen Besuch ab, der in erster Linie das fortgesetzte Interesse der Administration an Iran dokumentieren sollte. Bowles schätzte im Anschluss die Situation als „kontrollierbar“ ein. ${ }^{2}$ Tatsächlich war diese Stabilität nur von kurzer Dauer. Im März berichtete die Botschaft erneut von einer niedergeschlagenen Stimmungslage des Monarchen, der sich bitter über die Undankbarkeit und Vernachlässigung seitens der USA beschwerte. Angesichts des fehlenden Verständnisses für seine exponierte und gefährdete Position als Folge seiner pro-westlichen Haltung und der ausbleibenden Zusagen über größere Militärhilfe sei er womöglich gezwungen, als Oberkommandierender der Streitkräfte abzudanken. ${ }^{3}$ Bezeichnend für die sich wandelnde Stimmungslage in Washington war, dass diesen im Grunde altbekannten Drohungen nun erhöhte Aufmerksamkeit zuteil wurde. Auf Vorschlag des State Department wurde der Besuch des Schahs daher auf den April vorverlegt mit der Begründung, dass nur so eine Abdankung verhindert werden könne, die zwangsläufig in politischem Chaos resultieren würde. $^{4}$ Wenigstens konnte Bob Komer vorerst vereiteln, dass das geplante MAP-Programm

\footnotetext{
${ }^{1}$ Amini wehrte sich erfolgreich gegen die vom Schah geforderten Kabinettsänderungen, unterstützt vom SAWAKDirektor Pākrawān. Holmes kommentierte: „Amini is in new relationship with Shah and there is hope that with latter's backing [...] reform program in this country can move ahead.“; Tehran \#A-123, 6.11.61, ,Iran General 10/28/61-11/9/61“; Tehran \#369, \#370, \#378, \#403, 10-22.11.61, ,Iran General 11/10/61-12/10/61', NSF, Countries, Box 116, JFKL.

${ }^{2}$ Bowles' Kurzbesuch ersetzte die zuvor geplante Visite Robert Kennedys. Vgl. Brubeck Memo, 30.1.62, CF 6063, 788.11/1-3062; vgl. Cairo \#1208, 15.2.62, 788.5/2-1562; Tehran \#630, 19.2.62, 788.5/2-1862, CF 60-63; Tehran \#A-3, 13.2.62, ,Iran General 1/19/62-2/28/62', NSF, Countries, Box 116; Komer Memo, 5.2.62, ,Staff Memoranda, Robert Komer 2/62‘, NSF, M\&M, Box 322; Cairo \#A-274, 17.2.62, encl. to Komer Memo, 24.3.62, ,Iran General 3/1/62-3/26/62‘, NSF, Countries, Box 116.

${ }_{3}^{3}$ Ein deprimierter Mohammad Rezā hatte seine Abdankungsabsichten während eines Skiausflugs mit Yatsevitch und dem britischen Botschafter geäußert. Vgl. Tehran \#693, 7.3.62, FRUS 61-63, XVII, S. 508-11; ebd., S. 508 $n 1$; Hughes Memo, 6.3.62, ,Iran, 1961-1962 [Folder 2 of 2]', NSF, RWK, Box 424, JFKL; Harrisons Bericht nach London in ähnlichem Tenor, allerdings mit dem Hinweis: „Of course there is a certain element of indirect blackmail of the Americans.“; Harrison to FO, No. 221, 5.3.62, PREM 11/3845, TNA:PRO.

${ }^{4}$ Botschafter Holmes empfahl die Vorverlegung des Besuchs. Die Bemerkungen des Schahs über eine mögliche Abdankung wurden dieses Mal so ernst genommen, dass GTI sogar zur Abfassung eines Memorandums über die möglichen Folgen angewiesen wurde. Offenbar wurden Berichte über den Gemütszustand Mohammad Rezās sogar Kennedy vorgelegt. Vgl. Holmes Letter, 4.3.62, 788.11/3-462, CF 60-63; Miner Memo, 12.3.62, ,I. The
}

R. Popp, Vor der Revolution, Globale Gesellschaft und internationale

Beziehungen, https://doi.org/10.1007/978-3-658-33888-6_5 
- AID, das State Department und das Pentagon hatten sich in der Zwischenzeit auf eine Größenordnung von \$330 Millionen geeinigt - dem Schah bereits vor dem Besuch mitgeteilt wurde, was ohne Zweifel eine neue iranische Kampagne gerichtet auf Erhöhung der in Aussicht gestellten Hilfe ausgelöst hätte. ${ }^{5}$

Kaum waren die Vorbereitungen des Staatsbesuchs angelaufen, zeigte sich jedoch, wie sehr sich die Haltung der Kennedy-Administration inzwischen der Schah-zentrierten Politik der Vorgänger-Administration angenähert hatte. Selbst Komer gab als primäre Devise für den Besuch aus, die Sorgen des Monarchen zu zerstreuen:

Last May the President approved a new policy aimed at supporting a reformist government in its effort to achieve a 'controlled revolution' rather than an uncontrolled one. This remains our basic aim, but an indispensable element is to keep the Shah committed to or at least tolerant of our efforts to this end. Once before he threatened to dump Amini, he might do so again. If he himself left the throne, it would be equally disastrous. Hence we regard his continued presence and support of the Amini (or like) government as indispensable to Iranian stability over the next several years. There is no feasible alternative which would serve US interests as well. ${ }^{6}$

Die amerikanische Iranpolitik hatte sich von der Idee einer konsequenten Unterstützung einer Reformregierung in Iran - notfalls gegen die Interessen des Schahs - mit dem Ziel, eine alternative politische Synthese alternativ zur höfischen Autokratie zu schaffen, verabschiedet. Im Frühjahr 1962 ging es dagegen nur noch darum, eine gemäßigte, vornehmlich ökonomische Reformanstrengung mit der nun kaum noch in Frage gestellten monarchischen Prärogative in Einklang zu bringen. ${ }^{7}$ Komer wollte den Schah überzeugen, dass ,we vigorously support him“ sowie versuchen, durch verschiedene Präsentationen über die militärischen Kraftverhältnisse in globaler und regionaler Hinsicht und eine Bekräftigung der bestehenden Hilfszusagen der USA

Shah \& Court (and Govt) 1962‘, Box 4; Talbot Letter, 9.3.62, ,I-C. Corresp[...] 1962‘, NEA, GTI: RIAD, 195863, Box 5; ähnlich Harrison to FO, No. 220 und 221, beide 5.3.62, PREM 11/3845, TNA:PRO zur Vorverlegung des Staatsbesuchs vgl. Battle Memo, 8.3.62, FRUS 61-63, XVII, S. 516ff.; siehe ebd., S. 511 n3, 516 Anm. o.N.

${ }^{5}$ Vgl. ebd., S. 518 n7; die Antwort auf NSC Action 2447 war Anfang März erfolgt und plädierte wie der Steering Group Report für eine Reduzierung der Sollstärke der iranischen Streitkräfte um $25 \%$ und ein auf fünf Jahre angelegtes MAP-Program in Höhe von maximal \$330 Millionen - dies waren zwar \$30 Millionen mehr als die Steering Group vorgesehen hatte, blieb aber dennoch weit unter Holmes‘ Forderungen, die in der Zwischenzeit sogar auf eine Größenordnung von \$421 Millionen angewachsen waren. Vgl. Hamilton Memo, o.D. [8. bzw. 9.3.62], ebd., S. 512-6; State \#624, 16.3.62, ebd., S. 532f., und ebd., S. $533 n 1$, $n 2$; Holmes Letter, 5.2.62, ,I-C. Corresp[...] 1962‘, Box 5; Miner Memo, 6.2.62, ,Office Memoranda, 1962‘, NEA, GTI: RIAD, 1958-63, Box 4; die NEA-Entwürfe in ,3-A/1 [...] (MAP), Jan. 1- June', Box 6, ebd.; Komer hatte Kennedy empfohlen, vorerst keine endgültige Entscheidung über die Größe der zukünftigen Militärhilfe zu leisten, nicht zuletzt da mit zusätzlicher notwendiger Wirtschaftshilfe für den 3. Siebenjahresplan in Höhe von etwa \$400-600 Millionen zu rechnen war. Vgl. Komer Memo, 9.3.62, ,Iran Subjects, Shah Visit 1/31/62-3/20/62‘, NSF, Countries, Box 117, JFKL; Komer hatte bereits zuvor angemerkt, ein \$300 Millionen-Programm entspreche ungefähr \$50 Millionen per Jahr. Der jährliche Beitrag zwischen 1956 und 1961 betrug \$62 Millionen, und somit beinhalte das neue Programm im Grunde nur eine geringfügige Kürzung der MAP-Hilfe. Vgl. Komer Memo, 19.1.62, ,RWK Chron, Jan.-June 1962, Box 2‘, DSDUF, LBJL; vgl. Kitchen Memo, 2.2.62, ,MAP, Steering Group Report [Folder 2 of 2]', NSF, RWK, Box 432, JFKL.

${ }^{6}$ Komer Notes, 20.3.62, ,Staff Memoranda, Robert Komer 3/62‘, NSF, M\&M, Box 322, JFKL; Assistant Secretary Talbot vertrat in einem Treffen der NSC Standing Group eine ähnliche Meinung: ,we very much need the Shah in the next few years."; NSC Standing Group Meeting, 23.3.62, FRUS 61-63, XVII, S. 539f.

${ }^{7}$ William Polk vom Policy Planning Council brachte diesen Ansatz auf den Punkt: „, The most striking fact about Iranian development is that it depends upon a mere handful of people. [...] If we undermine the Shah's confidence in them - he already distrusts them as potential enemies - we may do terrible damage to our program."; Polk Memo, 29.3.62, ,Iran', Lot 69 D 121, S/P Records: 1962, Box 216. 
die diffusen externen Bedrohungsvorstellungen Mohammad Rezās zu revidieren. Der Schah sollte von der eigentlichen Hauptbedrohung durch indirekte Aggression überzeugt werden, deren Grundvoraussetzung, wirtschaftliche und soziale Instabilität, durch extensive Aufrüstung noch verstärkt werden würde. ${ }^{8}$ Während für Komer der Schahbesuch ,largely an exercise in psychotherapy“ war, sah man gerade auf Seiten des State Department eine Gelegenheit, den reformistischen Interventionismus der Administration weiter zurückzudrängen. Die Diskussionen über die tatsächliche strategische Lage Irans hatten zu der Einsicht geführt, dass es im Grunde keine geeigneten Maßnahmen gab, um den amerikanischen Beistandsverpflichtungen eine größere Glaubwürdigkeit zu verleihen. ${ }^{9}$ Damit wurde nicht nur die Grundidee in Frage gestellt, derzufolge man durch Demonstration der eigenen militärischen Kapazitäten und der eigenen Entschlossenheit, Iran im Ernstfall zur Hilfe zu kommen, wiederum den Schah überzeugen könne, auf eine weitere Aufrüstung der iranischen Streitkräfte zu verzichten. Zugleich wurden dadurch auch die wiederholten Mahnungen seitens NEAs und der Botschaft glaubwürdiger, dass Militärhilfe an Iran allein in ihrer politischen Funktion als Rückversicherung für den Schah dennoch im strategischen Interesse der USA sei. NEA hatte bereits im Vorfeld versucht, eine endgültige Festlegung der MAP-Obergrenze auf $\$ 330$ Millionen zu verhindern. Holmes' Botschaft plädierte weiterhin für ein weitaus größeres Programm, das inzwischen auf \$425 Millionen angewachsen war, sowie zusätzlich für weitere Zusagen bezüglich zukünftiger Wirtschaftshilfe. ${ }^{10}$ Komer warnte den Präsidenten, dass die seitens des Botschafters geforderte Erweiterung des MAP-Programms aus militärischer Perspektive unsinnig wäre und das Pentagon kaum in der Lage sei, auch nur \$300 Millionen zu begründen. Jede Steigerung der Militärhilfe würde ohnehin nur neue Forderungen des Monarchen generieren - zudem sei bereits jetzt ein Wirtschaftshilfeprogramm für Iran von \$400-600 Millionen absehbar: „We'll probably end up spending over a billion dollars in Iran during the Sixties; we ought (in the Shah's interest as well as ours) to spend it more effectively

\footnotetext{
${ }^{8}$ Komer Notes, siehe oben Anm. 6; Komer dachte an eine detaillierte Darstellung der strategischen Superiorität der USA gegenüber der Sowjetunion. Zur Bekräftigung der US-Beistandsverpflichtungen schlug Komer wieder einmal eine Aufwertung der CENTO bzw. alternativ eine Bekräftigung der Truman-Doktrin bzw. des bilateralen Abkommens von 1959, Zusagen im Falle konventioneller bzw. nuklearer Bedrohung und die Präpositionierung von militärischer Ausrüstung in Iran oder der Golfregion vor. Vgl. Komer Memo, 24.3.62, ,Iran General 3/1/623/26/62“; Talbot Memo, 27.3.62, ,Iran Subjects, Shah Visit [...]”, NSF, Countries, Box 117, JFKL; Talbot Memo, 28.3.62, ,Office Memoranda, 1962‘, NEA, GTI: RIAD, 1958-63, Box 4; Rostow regte eine Demonstration des Strategic Army Corps für den Schah an. Vgl. NSC Standing Group Meeting, 23.3.62, FRUS 61-63, XVII, S. 539f. ${ }^{9}$ Komer Memo, 21.3.62, ,Iran Subjects, Shah Visit [...]', NSF, Countries, Box 117, JFKL; gemeinsame militärische Planungen mit Iran würden ohne Zweifel Forderungen nach erheblich größerer Militärhilfe auslösen, die Präpositionierung von Ausrüstung konfligiere womöglich mit dem iranisch-sowjetischen Vertrag von 1921 und ohnehin seien die beiden verfügbaren Divisionen des Strategic Army Corps nicht für den Einsatz in Iran vorgesehen. Selbst McGeorge Bundy gestand nun ein ,that he was becoming quite sympathetic with the Shah. Everyone agreed that the Shah had no real defense against Soviet attack."; NSC Standing Group Meeting, 23.3.62, FRUS 61-63, XVII, S. 541ff.

${ }^{10}$ Vgl. Miner Memo, 7.3.62, ,3-A/1 [...] (MAP), Jan. 1- June‘, NEA, GTI: RIAD, 1958-63, Box 6; im Anschluss an den Bowles-Besuch hatte die Botschaft verschiedene dabei angerissene Themen aufgegriffen und weitere Argumente für das ausgedehntere MAP-Programm konstruiert, darunter den Ausbau des Hafens von Bandar 'Abbās. Hintergrund dieses Bowles-Vorschlags war die Öffnung alternativer Handelsrouten für Afghanistan, um einer Satellitisierung des Landes entgegenzuwirken. Daher drängte Bowles auf explizite Transit-Garantien Irans. Das vorläufige MAP-Programm sah tatsächlich einen Ausbau des Hafens vor. Vgl. Hamilton Memo, o.D. [8. bzw. 9.3.62], FRUS 61-63, XVII, S. 512-6.
} 
than the billion or so already invested since World War II.“11

Komers Warnungen, bei aller Konzentration auf Mohammad Rezās Prioritäten die über das rein Militärische hinausgehenden Teile des Reformpakets nicht aus den Augen zu verlieren, wurden jedoch nur bedingt berücksichtigt. Die Vorbereitungen des Besuchs konzentrierten sich letztlich auf eine erneute Bekräftigung der amerikanisch-iranischen Allianz, eine persönliche Rückversicherung des Schahs und verschiedene Strategien, zu erwartenden iranischen Forderungen nach größerer Militärhilfe entgegenzutreten. ${ }^{12}$ Allein Kenneth Hansen versuchte vergeblich, auf den Widerspruch zwischen der Konzentration auf die Befindlichkeiten des iranischen Monarchen und der offiziellen US-Iranpolitik nach Vorgabe des Iran Task Force Report hinzuweisen: „The briefing papers concentrate on the necessity for 'unselling' the Shah from a bigger and more glamorous military package. I believe there should be far greater stress on 'selling' the Shah on the need for moving faster on the economic and reform front." 13

Letztlich stellte diese Neuorientierung auch eine Rückwirkung der bereits seit Längerem geführten Debatten über die Gesamtstrategie gegenüber der Peripherie dar. Grundsätzlich bestand die Schwerpunktsetzung auf die Förderung reformerischer Maßnahmen in den Gesellschaften der Dritten Welt, in erster Linie intendiert als Prävention gegen kommunistische Subversion, weiterhin fort. Bereits während der Debatten über die Reform der US-Militärhilfe im Sommer 1961 aber war Iran als eines der sogenannten ,double threat countries“ identifiziert worden, was Staaten kennzeichnete, die sowohl aufgrund rapiden sozialen Wandels und Modernisierungsdrucks in ihrer inneren Stabilität gefährdet, zugleich aber aufgrund ihrer geografischen Lage entlang des sino-sowjetischen Kerngebiets direkter (militärischer) Bedrohung ausgesetzt waren. Diese Doppelfunktion schränkte somit die Anwendbarkeit der neuen strategischen Ansätze in der Peripherie insofern ein, da die unmittelbare Nähe militärischen Machtpotentials der kommunistischen Führungsmächte eine ausschließliche Konzentration auf sozio-ökonomische Reformen in diesen Staaten bzw. eine begleitende

\footnotetext{
${ }^{11}$ Komer Memo, 6.4.62, ,Iran Subjects, Shah Visit [...]', NSF, Countries, Box 117; Komer warnte dagegen vor der Gefahr, dass ein noch vages MAP-Programm zwangsläufig zu erhöhten Erwartungen seitens des Schahs führen würden. Ohnehin sei nicht endgültig festzulegen, wieviel „,military baksheesh“ überhaupt vonnöten sei, um dessen Wünsche zu befriedigen: ,even Holmes admits that no one can tell whether $\$ 300, \$ 330, \$ 424$, or $\$ 500$ million will do the job.“; vgl. Komer Memo, 20.3.62; Komer Memo, 22.3.62; Komer Memo, 30.3.62, ,Staff Memoranda, Robert Komer 3/62', NSF, M\&M, Box 322; Komer Memo, 2.4.62, ,Iran, 1961-1962 [White House Memoranda]', NSF, RWK, Box 424, JFKL; Komer Memo, 28.3.61, FRUS 61-63, XVII, S. 548f.

${ }^{12}$ Komer betonte, die Kritik seitens Irans, man erhalte anders als die Türkei oder Pakistan nur veraltetes Material, sei unberechtigt. Tatsächlich würden durch den Plan sämtliche, noch aus dem Weltkrieg stammenden Fahrzeuge ersetzt. Neben 26 leichten Düsenjägern sah es zudem die Einführung von 112 gepanzerten Mannschaftstransportwagen vom Typ $M$-113, vier $C$-130-Transportflugzeugen und 20 Helikoptern vor sowie die Verdoppelung der Munitionsbestände. William Bundy hatte vorgeschlagen, Änderungen am Programm zuzulassen, insbesondere hinsichtlich der zu erwartenden Wünsche nach Bau eines weiteren Flugfelds im Nordosten Irans bzw. nach der Aufstockung auf vier Staffeln von Jagdflugzeugen des Typs F-5 und nach einem Radarfrühwarnsystem. Entgegenkommen in dieser Frage sollte dann durch Streichung der bislang vorgesehenen Fregatten und Minenräumboote aus dem MAP-Programm kompensiert werden. Vgl. Komer Memo, 4.4.62; Komer Memo, 6.4.62, ,Staff Memoranda, Robert Komer 4/62‘, NSF, M\&M, Box 322; Komer Memo, 9.4.62, ,Iran Subjects, Shah Visit [...]', NSF, Countries, Box 117, JFKL; Note, 8.2.62; Miner Memo, 9.4.62, ,Office Memoranda, 1962', NEA, GTI: RIAD, 1958-63, Box 4; Taylor Memo, 14.3.62; Memos for the Record [MemCon], 2.4./9.4.62, FRUS 61-63, XVII, S. 531f., 570, 582f.

${ }^{13}$ Hansen Memo, 7.4.62, ebd., S. $580 \mathrm{ff}$.
} 
vorwiegende Ausrichtung der jeweiligen Streitkräfte auf Zivilhilfe als zu riskant erscheinen ließ. Stärker noch als in eindeutiger peripheren lateinamerikanischen oder afrikanischen Staaten war die Selbsteinschätzung der eigenen militärischen Stärke durch die Führungen dieser Staaten ein wichtiger Parameter für die Bestimmung der eigenen außenpolitischen Positionierung, unabhängig von der eigentlichen Frage, wie es um die tatsächliche Verteidigungsfähigkeit im Falle eines sowjetischen bzw. chinesischen Angriffs bestellt war. Mitte 1961 noch hatte eine Majoritätsmeinung die Auffassung vertreten, Iran stelle unter den „doppelt bedrohten“ Staaten der Peripherie so etwas wie einen Sonderfall dar. Die Konzentration auf die internen Probleme im Kontext der Iran Task Force hatten eine Verkleinerung der iranischen Streitkräfte wünschenswert erscheinen lassen, sowohl in innenpolitischer Hinsicht wie auch mit Rücksicht auf die enormen Belastungen, die der aufgeblähte Militärapparat der iranischen Volkswirtschaft aufbürdete.

Bereits damals hatten Zweifler wie der Assistant Secretary of Defense for International Security Affairs Paul Nitze der Vorstellung eines iranischen „Sonderfalls“ widersprochen. Zentrales Ziel sei es, so Nitze, die Sowjets von einem Vorgehen gegen Iran abzuschrecken. Eine Verkleinerung der iranischen Streitmacht würde somit automatisch den Druck erhöhen, den sowjetischen Forderungen entgegenzukommen:

\begin{abstract}
Mr. Nitze challenged the view that Iran was essentially different from other of the doubly threatened areas. In the first place, he pointed out that it was important to attempt to deter the Russians, and in this connection the existence of Iranian forces obviously played a role. The alternative was to have small forces which would clearly increase the pressures on Iran to accommodate to the Soviets. But if the deterrent failed, he doubted that we would decide that Iran was sufficiently important to permit a policy decision to drop nuclear bombs on Moscow. Instead, he felt that we would want to be in a position to support Iranian troops, with nuclear weapons or otherwise, and thus this represented another reason for the existence of such forces. As to the internal problem raised of $\{b y$ ?; R.P. $\}$ the existence of larger forces in Iran, this essentially was a political problem which would have to be solved as best we could but which did not invalidate the other arguments for Iranian forces. ${ }^{14}$
\end{abstract}

Nitze fasste im Anschluss ein eigenes Papier zur Verteidigung Irans ab, in dem er seine Gedankengänge weiterführte. Angesichts der bürokratischen Widerstände gegen eine Verlegung strategischer US-Reserven in den Iran im Ernstfall und angesichts der Glaubwürdigkeitsdefizite hinsichtlich der bestehenden Kriegspläne mit dem Einsatz taktischer Atomwaffen durch US-Verbände und mit Blick auf die generelle konventionelle Unterlegenheit plädierte Nitze für eine Ausdehnung der nuklearen Teilhabe auf Iran. Er skizzierte ein unabhängiges Verteidigungskonzept für Iran, basierend auf der Präpositionierung taktischer

\footnotetext{
${ }^{14}$ MemCon, 26.5.61, FRUS 61-63, IX, S. 253; geschwärzte Teile freigegeben per FOIA-Antrag des Verfassers (NW 36529). Rusk und andere brachten während dieser Diskussion wieder die Idee einer Präpositionierung von US-Truppen in symbolischer Stärke, nach dem Vorbild Südkoreas, ins Spiel. Der ehemalige Außenminister Acheson sprach sich gegen die Idee aus: ,[...] there was a vast difference between the Iranian and European situation, primarily because Europe was vital to the U.S., and Iran, however important, was not."; Rusk warnte sogleich, ganz im Geiste der Dominotheorie, vor einer möglichen Kettenreaktion im Falle eines Verlustes Irans, während Nitze die Meinung vertrat: „Iran could be the key to the Middle East and the South.“
} 
Atomwaffen für die iranischen Streitkräfte, unter Obhut von technischem Personal aus den USA. Allerdings müsse Iran - hier wich der Plan von der westeuropäischen Praxis ab - die ausschließliche Entscheidungsgewalt über den Einsatz der Waffen haben. Gleichzeitig müssten die USA eventuelle taktische Gegenschläge Moskaus durch Drohungen mit einer Eskalation auf der strategischen Ebene unterbinden - dies würde Iran eine autonome, erfolgreiche Verteidigung im Falle eines sowjetischen Angriffs ermöglichen. Somit seien sowohl das Glaubwürdigkeitsproblem bezüglich der amerikanischen Garantie sowie das verwandte Problem der politischen Konsequenzen iranischer militärischer Schwäche potentiell gelöst. ${ }^{15}$ Wenig überraschend war, dass eine derart weitreichende Lösung wie die von Nitze vorgeschlagene auf wenig Gegenliebe stieß. McGhee antwortete vom Policy Planning Council, dass eine solche Maßnahme ebenso provokativ und angesichts des ,Nth Country“-Problems der Proliferation zudem gefährlich sei:

Providing such nuclear weapons to Iran would lead to very insistent demands for similar weapons all along the periphery. Pakistan would want them for defense against India, etc. Perhaps this would serve our long run interests, but we doubt it. Placing such weapons in Iran opens a whole range of possible proliferation not implied by stationing tactical nuclear weapons in Western Europe. Finally, putting such a command decision in Iranian hands gives them more command control over our actions than that which appears to be possessed even by our NATO allies. We doubt whether this is a prudent proposal. ${ }^{16}$

Während die Vorschläge Nitzes zur Nuklearisierung der iranischen Verteidigungsfähigkeit rasch verworfen wurden, blieben seine Feststellungen über die Bedeutung der Selbstwahrnehmung eigener militärischer Stärke für das strategische Kalkül der Frontstaaten an der Peripherie dennoch wirksam. Mit der Bestätigung der Position des Schahs als dominierendes inneriranisches Machtzentrum im Frühjahr 1962 und angesichts dessen nahezu obsessiver Konzentration auf alles Militärische wirkten Nitzes Argumente ohne Frage noch stichhaltiger als zuvor. ${ }^{17}$

In diesem Kontext ist auch die schrittweise Priorisierung der militärischen Aspekte der Counterinsurgency-Konzeption gegenüber den Ideen extern erzwungener, durchgreifender sozio-ökonomischer Reformen zu sehen. Die Rückbesinnung auf die tradierten Bündnispartner entlang der Peripherie seit Ende 1961 ließ eine Ende bzw. wenigstens einer Verlangsamung des

\footnotetext{
${ }^{15}$ Study ,An Additional Alternative for the Military Defense of Iran Against Overt Soviet Union Attack', o.D., encl. to Nitze Memo, 7.7.61; diese bislang unbekannte Studie wurde deklassifiziert infolge eines FOIA-Antrags des Verfassers (NW 21608). Nitze selbst nannte sie eine ,imaginative suggestion about the recurring problem of creating a credible strategy for defending non-Communist countries contiguous to the bloc against overt attack." \{Zitat aus Memo an McGhee\}; McQuade Memo, 12.4.61, ,Iran, Revised Military Strategy, 1961', Box 121, Nitze Papers, LOC.

${ }^{16}$ McGhee Letter, 2.8.61, ,Iran 1958-1961', Lot 67 D 548, S/P Records: 1957-1961, Box 141.

17 Die Debatten über die militärstrategische Position Irans hielten auch im Anschluss an. Im Laufe von 1962 wurden drei unterschiedliche „War Games“" zu Iran durchgeführt, am MIT in Boston, in RAND und im Pentagon. Rostow koordinierte vom Policy Planning Council aus die Nachfolgetreffen. Vgl. DOD, Final Report of PoliticoMilitary Game-Olympiad I-62: Mid-East, 3.12.62, DDRS: CK2349262361; vgl. Rostow Memo u.a., 29.6.62, ,Iran 1962 000.1---092‘, OASD/ISA Files: General and Country, 1962, Box 89, RG 330; Vgl. Rostow Memo, 29.6.62, ,Office Memoranda, 1962‘, NEA, GTI: RIAD, 1958-63, Box 4; Bowling Draft Memo, 13.7.62, , 3-A/1. IRAN War Game‘, Box 6, ebd.
} 
Reformeifers erwarten, was wiederum das erneute Ausbrechen sozialer Unruhe nach dem Muster der Ereignisse in Teheran vom vergangenen Frühling wahrscheinlich werden ließ. Die primäre Antwort auf solche Unruhe war aber nun nicht mehr das Durchsetzen eines reformistischen Programms in diesen Gesellschaften nach dem Muster der Durchsetzung Aminis bzw. die damals auch angedachte politische Partizipation der sich widersetzenden städtischen Mittelschichten, sondern die Unterstützung der staatlichen Sicherheitskräfte bei der gewaltsamen Unterdrückung bzw. Unterminierung der jeweiligen Opposition. Während der internen Debatten über die geeignetste Umsetzung der verschiedenen CounterinsurgencyAnsätze hatte sich bereits eine Konzentration auf die Polizeikräfte bzw. die Geheimdienste in den politischen Entitäten der Dritten Welt durchgesetzt, eine Tendenz, die durch die Vorgehensweise der im Januar 1962 eingesetzten Special Group (CI) noch verschärft wurde. Im Laufe des Jahres 1961 waren fünf Spezialisten der Public Safety Division im Rahmen des von der Vorgängeradministration ererbten OISP-Programmes beratend für die iranische Polizei tätig, wobei deren Tätigkeit zuvorderst auf eine Professionalisierung der Polizeiarbeit gerichtet war. Der für den Anlass des Schahbesuchs ebenfalls nach Washington zurückgekehrte Holmes drängte auf eine Ausdehnung dieser Kooperation. Der Special Group (CI) gegenüber forderte er die Lieferung von umfangreicher Ausrüstung für Straßenkämpfe, um die iranischen Sicherheitskräfte in die Lage zu versetzen, die auch für die Zukunft zu erwartenden Demonstrationen unter Kontrolle $\mathrm{zu}$ halten. Allerdings sollten auf diesen Ausrüstungsgegenständen sämtliche Hinweise auf amerikanische Herkunft beseitigt werden eine Reaktion auf die Ereignisse vom Mai 1961, als die Polizei in Teheran Material mit AIDEmblemen zur Niederschlagung der Unruhen benutzt hatte. ${ }^{18}$

In Reaktion auf das Drängen Präsident Kennedys waren zudem die Bemühungen seitens der US-Armee zugunsten einer stärkeren Ausrichtung auf Counterinsurgency intensiviert worden. Die Teheraner Botschaft regte im April eine Intensivierung der Zusammenarbeit mit der iranischen Armee in diesem Feld und die Entsendung von amerikanischen Ausbildungsexperten an. Diese sollten u.a. den voranschreitenden Aufbau eines eigenen iranischen Special Warfare Battalion unterstützen. Für die Zukunft, so der Rat der Botschaft, sei auch eine direkte US-Unterstützung für die effektive Organisation und Ausbildung der „Nationalen Widerstandsorganisation“ denkbar, der vom Schah in der Mosaddeg-Zeit gegründeten, ultranationalistischen Milizorganisation. ${ }^{19}$ Angesichts der Bereitschaft einiger

\footnotetext{
${ }^{18}$ Unmittelbar nach Ausbruch der Unruhen wurde das Country Team informiert, dass ,,[...] the Clasped Hands insignia on all of the riot control equipment provided to Iran had been removed."; vgl. Summary Notes, 4.5.61, 16.11.61, ,5. Country Team Meetings 1961‘, NEA, GTI: RIAD, 1958-63, Box 3; vgl. Minutes Special Group Meeting, 26.4.62, ,SPECIAL GROUP (CI) 1/1/62 Thru 7/31/62', Lot 68 D 451, SG (CI) Records, Box 1; ein internes Memorandum der Special Group (CI) bezeichnete die seit 1954 in Iran tätige Beratermission der Public Safety Division als ,outstanding success“. In den acht vorausgegangenen Jahren habe man die gesamte Führungsstruktur der 24.000 Mann starken städtischen Polizei reorganisiert, moderne Ermittlungsmethoden und Kriminaltechniken vermittelt und zudem bereits eine Einheit zur Kontrolle von Massenaufläufen- und Demonstrationen ausgebildet und diese mit Wasserwerfern und Transportwagen ausgerüstet. Vgl. Memo for Taylor, 17.4.62, ebd.

${ }^{19}$ In Reaktion auf Kennedys Beschwerde vom Januar 1962 hatte die U.S. Army unter anderem den Aufbau von vier, auf verschiedene Weltregionen zugeschnittenen Cold War Augmentation Detachments (die für den Einsatz
} 
amerikanischer Stellen, selbst mit den bedenklichsten Organen des Schah-Regimes zu kooperieren, konnte die weiterhin fortbestehende enge Kooperation auf nachrichtendienstlicher Ebene eigentlich nicht überraschen. SAWAK-Direktor Pākrawān war einer der wichtigsten Informanten über die Entwicklung der iranischen Innenpolitik und die von der EisenhowerAdministration vererbte Kooperation mit der als effizienteste unter den Sicherheitsorganen in Iran eingeschätzten Institution wurde fortgesetzt. Allerdings entschloss man sich, eine gewisse Distanz einzunehmen. Offenbar in dieser Zeit wurde die seit 1955 in Iran tätige und im SAWAK-Hauptquartier stationierte CIA-Trainingsmission abgezogen - ein CIA liaison team aber blieb vor Ort. Zudem wurden viele, bislang von der CIA ausgeführte Aufgaben von einem israelischen Team übernommen. CIA Station Chief Yatsevitch zufolge installierte der Mossad im Rahmen der israelisch-türkisch-iranischen Kollaboration auf dem Geheimdienstsektor (TRIDENT) eine der modernsten Anlagen zur Telefonüberwachung für ganz Iran. ${ }^{20}$ In der Special Group (CI) analysierte man, dass die einzige wirkliche Gefahr für das Schah-Regime in städtischen Aufständen bestehe, eine Gefahr, die durch weitere US-Unterstützung für die iranischen Sicherheitsorgane in Schach gehalten werden könne. Die städtische Opposition war somit in wenigen Monaten von dem zentralen, nur im Rahmen einer politischen Gesamtstrategie lösbaren Problem zu einem beherrschbaren, fast marginalen Betätigungsfeld für Counterinsurgency-Ansätze geworden. ${ }^{21}$

Eigentlicher Auslöser für Holmes`Initiative zur Verbesserung der Polizeispezialkräfte waren

im der Nahostregion vorgesehene Gruppe war in der Westdeutschland stationiert) vorangetrieben, bestehend jeweils aus einer ca. 1.300 Mann starken Gruppe von Spezialkräften und dazugehörigen logistischen und nachrichtendienstlichen Zusatzeinheiten. Sie sollten vonseiten der MAAGs in den verschiedenen Staaten angefordert werden können u.a. mit dem Ziel des, ,denial of civilian support to dissident elements and rallying this support to the government being assisted.“; vgl. Kennedy Memo, 11.1.62, FRUS 61-63, VIII, S. 235f.; Saunders Memo, 16.4.62; U.S. Army Special Warfare Center Headquarters (Fort Bragg), Training Memorandum No. 33, 22.12.61, ,Counterinsurgency, Engineers, 1962-1963‘, NSF, RWK, Box 412, JFKL; vgl. die Reaktion der USBotschaft in Teheran auf die sog. CA-2108-Anfrage in Tehran \#A-268, ,Anti-Guerrilla Training and Civic Action (CA-2108), 19.4.62, 788.5/4-1962, CF 60-63; vgl. KREPINEVICH, JR., Army and Vietnam, S. 103-7; die Nationale Widerstandsorganisation diente als pro-monarchische Miliz im Falle von Aufständen, zur Bekämpfung von Guerillas und als Nukleus einer Stay Behind-Armee zur Ausführung von eigenen Guerillaaktivitäten im Falle eines sowjetischen Einmarsches. Einem Papier aus dem Umfeld der Special Group (CI) zufolge erhielt die Organisation zu dieser Zeit keine ,,direkte US-Unterstützung“; vgl. Memo for Taylor, 17.4.62, ,SPECIAL [...] 7/31/62 ‘, Lot 68 D 451, SG (CI) Records, Box 1; der Zusammenhang des Aufbaus der Organisation mit ähnlichen Aktionen in Westeuropa im Anschluss an NSC 10/5 vom Oktober 1951 ist naheliegend. Vgl. auch oben, S. 93, Anm. 218 und S. 96, Anm. 232.

${ }^{20}$ Vgl. Memo for Taylor, 17.4 .62 (vorherig. Anm.); der in dieser Version enthaltene Satz „It $\{S A W A K$; R.P. $\}$ has received technical assistance from CIA in the past.", wurde in der für die vorliegende Arbeit 2004 freigegebenen Version des Memos geschwärzt. Siehe die Version in ,Iran, 1961-1962 [Folder 2 of 2]', NSF, RWK, Box 424, JFKL; Gratian Yatsevitch OHI, 5.11.88, OHIC; S. I-116f.; zu TRIDENT vgl. CIA, ,Israel: Foreign Intelligence and Security Services‘, März 1979, Asnād 11, S. 24; zum Abzug des Training Team vgl. GASIOROWSKI, Client State, S. 117f., 124; ders., Security Relations, S. 155; Kermit Roosevelt kommentierte später die ,excellent though informal“ israelisch-iranischen Beziehungen und behauptete, dass ,certain Israeli friends discreetly joined the CIA in helping to organize and give guidance to a new Iranian security service.“; vgl. RoOSEVELT, Countercoup, S. 9; Ben-Gurion unternahm einen einwöchigen Geheimbesuch in Teheran im Dezember 1961. Vgl. Yatsevitch Memo, 14.12.61, encl. to Tehran \#293, 20.12.61, 788.11/12-2061, CF 60-63; Pollack, Persian Puzzle, S. 139, stützt seine apologetische Darstellung u.a. auf die Behauptung, dass die USA, ,never even sold Iran tear gas or riotcontrol equipment until late in the revolution" und insgesamt ,was not culpable in the crimes committed by the shah's regime against the Iranian people."; zumindest was den Aufbau von Spezialpolizeikräften für den Straßenkampf und die Bereitstellung umfangreichen Ausrüstungsmaterials für diese Zwecke angeht, kann die vorliegende Untersuchung diese Behauptung vollständig widerlegen.

${ }^{21}$ Vgl. Memo for Taylor, 17.4.62, oben Anm. 19; vgl. auch Rockwell Letter, 5.6.62, ,3-A/1. Military Contingency Planning', NEA, GTI: RIAD, 1958-63, Box 6. 
die Ereignisse im vorangegangenen Januar an der Teheraner Universität gewesen. Ein kurzer Solidaritätsstreik der Teheraner Studenten für einige von der Universität verwiesene Kommilitonen hatte sich zu einer Großdemonstration auf dem Campus ausgeweitet. Iranische Sicherheitskräfte stürmten daraufhin das Universitätsgelände und gingen dabei mit großer Brutalität vor. Mindestens zwei Studenten kamen ums Leben. Offenbar war selbst die Nationale Front - die die anfänglichen Demonstrationen noch unterstützt hatte - vom Ausmaß der Zusammenstöße überrascht. ${ }^{22}$ Einige Indizien sprachen für eine bewusste Eskalation seitens einiger rechtskonservativen Kreise und insbesondere des ehemaligen SAWAK-Chefs Tajmur Baxtijār, offenbar mit dem Ziel, die Regierung Amini zu stürzen und den Schah zur Berufung eines rechtsgerichteten bzw. autoritären Nachfolgers zu bewegen. Amini nutzte die Gelegenheit, sowohl die verschiedenen Führungsfiguren der Nationalen Front als auch gleichzeitig zahlreiche Protagonisten der rechten Opposition wie z.B. Asadollah Rašidijān, festsetzen zu lassen. Zudem setzte er beim Schah die sofortige Exilierung Baxtijārs durch, der das Land innerhalb von 48 Stunden verlassen musste. ${ }^{23}$ Bezeichnend für die geänderte Stimmungslage in Washington war, wie wenig diese Ereignisse - sie waren im Grunde gleichbedeutend mit einem endgültigen Bruch zwischen Amini und der städtischen Opposition - überhaupt Resonanz fanden. Aus Perspektive der Zeit der Abfassung des Task Force Report vom Mai 1961 wäre diese Entfremdung zwischen Opposition und der im Grunde mit dem Ziel der Kooptierung der Mittelschichten eingesetzten Reformregierung sicherlich als die am wenigsten wünschenswerte Entwicklung eingeschätzt worden. Nun aber pries man die Entschlossenheit und Kaltblütigkeit Aminis, der durch sein Auftreten die volle Unterstützung des Schahs gewonnen habe. Die Nationale Front dagegen habe trotz Einsatz aller ihr zur Verfügung stehenden Mittel eine empfindliche Niederlage empfangen und ihre Bedeutung als

\footnotetext{
${ }^{22}$ Vgl. Tehran \#537, 21.1.62, ,Iran General 1/19/62-2/28/62‘, NSF, Countries, Box 116, JFKL; innerhalb GTIs hielt man die Pläne für unnötig: ,the riot control capability of the Tehran police has already been significantly strengthened by assistance which we have provided."; Rockwell Memo, 7.5.62, ,3-A. Civil (National) Police", NEA, GTI: RIAD, 1958-63, Box 6; man hoffte aber, die CIA werde die Finanzierung übernehmen. ARMISHMAAG berichtete, die großen Zerstörungen innerhalb der Universität seien auf die Spezialeinheiten der Fallschirmjäger zurückzuführen, die iranische wie auch ausländische Studenten wahllos zusammengeschlagen und zugleich das Eigentum der Studenten geplündert hätten. Vgl. USARMA Tehran \#197231, 7.1.62, 788.00 (W)/12762, CF 60-63.

${ }_{23}$ Amini zeigte sich in Gesprächen mit Holmes von einer gemeinsamen Verschwörung der Nationalen Front mit den rechten Kreisen überzeugt und sprach von eindeutigen Beweisen für Baxtijārs Beteiligung. Yatsevitch zufolge war Baxtijār über die erzwungene Ausreise enorm verbittert und diese Demütigung einer der Hauptursachen für seine spätere Kollaboration mit Schah-feindlichen Kräften im Ausland. Amini behauptete öffentlich eine Beteiligung der sowjetischen Botschaft. Offenbar versuchte Amini, die zu dieser Zeit wieder angelaufenen iranisch-sowjetischen Verhandlungen über die bereits erwähnte unilaterale Deklaration Irans bezüglich der Nichtstationierung von Raketen auf dem eigenem Territorium zu sabotieren. Vgl. Tehran \#A-174, 3.1.62, 788.00/1-362; Tehran \#573, 30.1.62, 788.00/1-3062; USARMA Tehran \#199407, 3.2.62, 788.00(W)/2-362, CF 60-63; vgl. Gratian Yatsevitch OHI, 5.11.88, OHIC; S. I-69f.; über die Januar-Zusammenstöße waren bald alle denkbaren Verschwörungstheorien im Umlauf. Innerhalb der nationalen Front gab es Vorwürfe an einige der bekanntesten Führer, diese hätten insgeheim mit Baxtijār kooperiert, ein Vorwurf, der wohl in erster Linie an Sāpur Baxtijār, den Cousin des Ex-SAWAK-Chefs, gerichtet war. Vgl. ebd., S. I-88f.; Marshall Memo, 7.6.62, Asnād 23, S. 126ff.; KATOUZIAN, Struggle for Power, S. 233ff., glaubt offenbar an eine Zusammenarbeit von Teilen der Nationalen Front mit Baxtijār. Dementiert bei LADJEVARDI, Xāterāt-e Šāpur Baxtijār, S. 55-59; wahrscheinlicher ist ein Versuch Baxtijārs, die entstandene Unruhe für seine politischen Ziele auszunutzen. So fand während der Vorgänge in der Universität eine „spontane“ Kundgebung offenbar dafür bezahlter Demonstranten vor Baxtijārs Haus und am darauffolgenden Tag eine weitere im Basar statt, die eine Rückkehr des Generals in die Politik forderten. Vgl. Tehran \#358, 3.2.62, 788.00/2-362; das Country Team bewertete eine Machtübernahme Baxtijārs aus Sicht der US-Interessen als ,, a last resort“" und empfahl Widerstand gegen derlei Absichten. Vgl. Rockwell Letter, 5.6.62, ,3-A/1. Military Contingency Planning', NEA, GTI: RIAD, 1958-63, Box 6.
} 
politischer Faktor habe in der Folge abgenommen. ${ }^{24}$ Eine Einschätzung der verbliebene Stärke der nationalistischen Opposition durch die Botschaft vom Dezember 1961 kam zu einer ernüchternden Feststellung: „In terms therefore of objective power, that is, the power to bring down the government, or to change substantially its policies, the nationalists are for the moment largely ineffective. “25 Von den ambitionierten Zielen der Task Force war kaum noch etwas übrig geblieben: Eine Policy Directive des State Department vom März 1962 gab dann auch als primäres Ziel der US-Politik gegenüber Iran aus, eine pro-westliche Regierung zu sichern ,[...] with the will and ability to resist Soviet pressures with a broader political base and developed channels of articulation outside existing elites and would-be elites." Dieser Halbsatz war der letzte Bezug auf die früheren Ideen über den Aufbau einer „new political synthesis“ in Iran. Die konkret in der Direktive genannten Maßnahmen aber hätten ebenso aus der EisenhowerÄra stammen können. ${ }^{26}$

Diese geänderte Gesamthaltung der Administration zur Frage der Partizipation breiterer Bevölkerungsschichten an der politischen Willensbildung in Iran spiegelte sich auch in den Briefing Books für den Präsidenten wieder, die zum Anlass des Schah-Besuchs erstellt wurden. Das zentrale Gesprächspapier für Kennedy begann mit einer umfangreichen Erklärung über den Gemütszustand des iranischen Monarchen und die Notwendigkeit, den Schah bezüglich der amerikanischen Unterstützung rückzuversichern. Der Schwerpunkt lag dabei auf der Zusicherung militärischen Schutzes seitens der USA und auf Zusagen hinsichtlich des bereits intern vereinbarten MAP-Programms. Das Reformprogramm nahm zwar ebenfalls einen bedeutenden Platz ein. Allerdings warnten State Department wie Botschaft davor, den Ministerpräsidenten besonders zu erwähnen - stattdessen sollten die bislang erreichten Reformschritte ganz dem Engagement des Schahs zugeschrieben werden. ${ }^{27}$ Dagegen warnten

\footnotetext{
${ }^{24}$ Vgl. Tehran Despatch \#358 (vorherig. Anm.); ein (offiziell noch nicht deklassifizierter) Bericht der Botschaft über die Nationale Front vom Dezember 1961 hatte ein vernichtendes Urteil über die nationalistische Opposition gefällt. Insbesondere die Führungspersönlichkeiten der Front seien letztlich charakterlich für höhere Aufgaben nicht geeignet, einziger Zusammenhalt der Gruppe sei die gemeinsame Ausrichtung auf unrealistische öffentliche Positionen. Aminis Reformprogramm habe ihre politische Plattform unterminiert und sie hätten keinerlei Machtmittel, um die Regierung in Bedrängnis zu bringen. Die Nationale Front sei daher eine Partei ,, [...] in search of a demagogue": ,These internal weaknesses, added to the turmoil which would certainly surround their rise to power, make it most unlikely that the NF \{National Front; R.P.\} or any of the fringe nationalist groups could insure the stability and independence of Iran."; Marcy Memo, 5.1.62, ,14. Opposition Movements, 1962', NEA, GTI: RIAD, 1958-63, Box 7; Marcys Memo bezieht sich auf Tehran \#283, 11.12.61, bereits vor der Freigabe infolge eines FOIA-Antrags des Autors dieser Studie zu finden in den erbeuteten Botschaftsdokumenten. Siehe Asnād 20, 1, S. 60-70 (deklassifiziert im Juli 2016 nach FOIA-Antrag NW21608); Bowling bezeichnete die Front einen Monat später willens $\mathrm{zu},[\ldots]$ cooperate with communists and reactionaries to bring down a moderate reformist government and so obtain power."; im selben Memo bezeichnet Bowling Mosaddeg als Diktator; Bowling Memo, 23.1.62, 788.00/1-2362, CF 60-63; offenbar spielte die Amini-Regierung mit Überlegungen, die meisten Führer der Nationalen Front für einige Jahre zu inhaftieren. Ein Telegrammentwurf aus GTI empfahl aber die baldige Freilassung der moderaten wie unfähigen Führer, da andernfalls mit dem Aufstieg radikalerer Kräfte zu rechnen sei. Vgl. Draft Telegram, o.D. [Januar 1962], ,14. National Front, 1962‘, NEA, GTI: RIAD, 1958-63, Box 7.

${ }^{25}$ Tehran \#283, 1.12.61, Asnād 20, 1, S. 69.

${ }^{26}$ Von den Vorstellungen der „Aktivisten“ im Weißen Haus war kaum noch etwas in der Direktive enthalten. Vgl. State Policy Directive, ,PD/NEA-12.1: Strengthening of the Political Unity, Economic and Social Modernization, and National Integrity of Iran', 16.3.62, ,Iran, 1961-1962 (Folder 2 of 2)', NSF, RWK, Box 424, JFKL.

${ }^{27}$ Vgl. State Briefing Paper, 3.4.62, ,Shah [...] 4/14/62 Index-Tab III‘, NSF, Countries, Box 117, JFKL; das Paper warnte: „We must keep the Shah on our side. There are no feasible alternatives.“; die Botschaft hatte vor dem Besuch ausdrücklich geraten den Eindruck zu erwecken, that the United States not only has a great interest in Iran but also in Iran's monarch."; Amini sei am besten nicht zu erwähnen. Vgl. Tehran \#742, 20.3.62, ,CF 2082 [...]
} 
insbesondere Kenneth Hansen, aber auch Bob Komer, davor, bei aller notwendigen Rückversicherung für den Schah die zentrale Bedeutung der Amini-Regierung für die Reformanstrengungen zu unterschlagen. ${ }^{28}$ Was die inneriranische Opposition anging, so wurde sie im Vorfeld des Besuchs eigentlich nur noch als potentieller Störfaktor wahrgenommen. Man vereinbarte frühzeitig, Demonstrationen seitens der in Amerika ansässigen, gegen das Regime arbeitenden Studenten zu verhindern. ${ }^{29}$ Zudem wurde eine Anregung der Botschaft aufgegriffen, dieses Mal das öffentliche Erscheinungsbild des Schahs während des Staatsbesuchs etwas bewusster zu steuern. Holmes empfahl das Image des ,reformerischen Monarchen" als Leitbild - angesichts der Rolle des Hofs als eines der Haupthindernisse bei der Umsetzung der Reformen im Grunde ein Hohn. ${ }^{30}$ Das State Department bat daher, die iranische Delegation solle keine Uniformen tragen - auch auf die sonst üblichen ausgiebigen Einkaufstouren und das großzügige Verteilen von Geschenken solle dieses Mal verzichtet werden. Gleichzeitig bemühten sich die offiziellen Stellen wie auch das informelle PahlavistsNetzwerk um eine positive Berichterstattung während des Besuchs - offenbar mit zufriedenstellendem Erfolg. ${ }^{31}$

Die Gespräche während des Schah-Besuchs vom 10. bis 18. April 1962 konzentrierten sich erwartungsgemäß auf militärische Belange. Der Schah versuchte unter Hinweis auf die ihm zufolge zunehmende „Satellitisierung“ Iraks und Afghanistans ein größeres MAP-Programm zu begründen. Präsident Kennedy und die anderen Gesprächspartner Mohammad Rezās

April 1962, MISC‘, Lot 66 D 110, Conference Files, Box 284; Komer beschwerte sich allerdings später, die Briefing Books des State Department seien generell „crappy“ gewesen und man habe sie häufig gar nicht an den Präsidenten weitergegeben. Vgl. Robert W. Komer OHI, 11.8.87, OHIC, S. I-38, 44.

${ }^{28}$ Hansen beschwerte sich, dass die Briefing Papers die Rolle Aminis praktisch nicht erwähnten. Vgl. Hansen Memo, 7.4.62, FRUS 61-63, XVII, S. 580ff.; Komer unterstützte Hansens Position und warnte, der Besuch müsse mehr als nur die Rückversicherung des Schahs in militärischer Hinsicht erreichen. Der zweite Schwerpunkt müsse auf ,development and reform, together with improved counter-insurgency capabilities“ liegen; Komer Memo, 6.4.62, ,Iran Subjects, [...]4/9/62', NSF, Countries, Box 117, JFKL; auch Bowles, Justice Douglas und der ehemalige US-Botschafter John Wiley sekundierten in diese Richtung. Vgl. Wiley Letter, 23.3.62, ebd.; Bowles Memo, 10.4.62, ,CF 2082 [...] 1962, MISC', Lot 66 D 110, Conference Files, Box 284; Talbot Letter, 6.3.62, ,IC. Corresp[...] 1962‘, NEA, GTI: RIAD, 1958-63, Box 5.

${ }^{29}$ Vgl. NSC Standing Group Meeting, 23.3.62, FRUS 61-63, XVII, S. 541; Komer Memo, 21.3.62, , Iran Subjects [...] 3/24/62،, NSF, Countries, Box 117, JFKL; Beschwerden des Schahs über die Aktivitäten sollten aber mit Verweis auf die Meinungsfreiheit in den USA begegnet werden. Vgl. State Briefing Paper, 2.4.62, ,Iran Subjects: [...] Tab VI (8) ${ }^{6}$, NSF, Countries, Box 117, JFKL; bereits im Januar hatte es Verstimmungen wegen des Verhaltens der Polizei bei einer friedlichen Botschaftsbesetzung durch Studenten gegeben. Vgl. Sanjuan Memo, 25.1.62, 788.11/1-2562, CF 60-63; zu den iranischen Studenten in den USA und der oppositionellen Übernahme der dortigen Studentenorganisation im Jahr 1960 und der anschließenden Radikalisierung vgl. MATIN-ASGARI, Iranian Student Opposition, S. 36-42, 47ff.

${ }^{30}$ Vgl. Tehran \#742, vorherig. Seite, Anm. 27.

${ }^{31}$ Vgl. State \#642, 21.3.62, 788.11/3-1962; State \#645, 21.3.62, 788.11/3-2162, CF 60-63; Komer glaubte, dass man ,ought to be able to inspire a few more good articles [...]."; Komer Memo, 21.3.62, ,Iran [...] 3/24/62‘, NSF, Countries, Box 117, JFKL; die New York Times enthielt im März mehrere Artikel von Dana Adams Schmidt mit einer Schah-freundlichen Berichterstattung im Sinne des ,reformerischen Monarchen“. Siehe z.B. Schmidt, ,Shah Bolsters Regime in Iran: Support for Premier Amini Paves Way for Reform‘, 19.3.62, New York Times, S. 3; die Botschaft in Teheran zeigte sich ob des PR-Erfolges begeistert. Vgl. Rockwell Letter, 17.4.62; Holmes Letter, 26.4.62, ,I-C. Corresp[...] 1962‘, NEA, GTI: RIAD, 1958-63, Box 5; das Pahlavists-Netzwerk war wohl mitverantwortlich für die Organisation der Schah-freundlichen Berichterstattung. Der seitens des Regimes mit den PR-Maßnahmen beauftragte Osgood Nichols und auch Kermit Roosevelt wandten sich direkt an das Weiße Haus, um den Besuch zu einem Erfolg werden zu lassen. Vgl. Schlesinger Memo, 7.4.62, , Iran, Shah's [...] 4/14/62 [Folder 1 of 2]', NSF, RWK, Box 425, JFKL; offenbar war die PR-Firma Kaston, Hilton, Chesley, Clifford \& Atherton beauftragt worden. Richard Cottam kommentierte: ,the excellent press the Shah has received in this country suggests these funds are well spent.“; COTTAM, Competitive Interference, S. 60. 
während des Staatsbesuchs waren allerdings auf diese Argumentation im Detail vorbereitet tatsächlich hatten die Amerikaner offenbar aus geheimdienstlichen Quellen ein entsprechendes Briefing Book vonseiten der iranischen Luftwaffe erhalten und waren somit im Vorhinein über die iranische Position informiert. ${ }^{32}$ Im Kern kam die Gesprächstaktik des Schahs den Amerikanern sogar entgegen, da eine militärische Abwehr eines möglichen Angriffs der Nachbarstaaten eher kleinere und mobilere iranische Streitkräfte notwendig machte, so wie in den MAP-Plänen vorgesehen. ${ }^{33}$ Präsident Kennedy und Secretary of Defense McNamara drängten erfolgreich auf eine Einwilligung des Schahs zu einer Verringerung der Sollstärke der iranischen Streitkräfte im Gegenzug zu einem Modernisierungsprogramm. Wie zu erwarten, sträubte sich Mohammad Rezā aber gegen einzelne Details des vorgesehenen MAPProgramms, insbesondere gegen die aus seiner Sicht unzureichenden Stückzahlen an modernem Kriegsgerät. Auf amerikanischer Seite war man allerdings vorbereitet und akzeptierte tatsächlich waren die meisten Konzessionen bereits im Vorfeld beschlossen worden - einige der Änderungswünsche des Monarchen. ${ }^{34}$ Trotz der taktisch geschickten Handhabung des Schahbesuchs in Fragen der Militärhilfe hatte man sich letzten Endes zu einem MAP-Programm bekannt, das in den wichtigsten Details den zuvor noch als exorbitant empfundenen Vorschlägen von Botschafter Holmes vom September 1961 weitgehend entsprach. Holmes notierte hoffnungsvoll: „I feel sure the total cost is going to exceed our original recommendation made last September.“35

Letztlich war es John F. Kennedy selbst, der gegenüber dem Schah am nachdrücklichsten das

\footnotetext{
${ }^{32}$ Vgl. ,Consideration of Points Listed in Shah's Briefing Paper', encl. to Komer Memo, 11.4.62, ,Iran Subjects [...] 4/15/62‘, NSF, Countries, Box 117, JFKL; nicht völlig geklärt ist die Frage iranischer Interventionen in der Kurdenfrage Iraks. Offenbar gab es anfangs auch Überlegungen, eine Anti-Bārzāni-Koalition unter den kurdischen Stämmen zu fördern. Vgl. CIA Telegram, 10.7.62, ,Iran [...] 7/31/62‘, NSF, Countries, Box 116; das Talking Paper stellte fest, weder Afghanistan noch Irak, ,is even a semi-satellite“; State Briefing Paper, President's Talking Paper, 3.4.62, ,Shah Briefing [...]-Tab III‘, NSF, Countries, Box 117, JFKL; vgl. aber State Briefing Paper, ,The Motivations and Extent of Soviet Bloc Military Aid to Iraq and Afghanistan', 3.4.62, ,Iran', Lot 69 D 121, S/P Records: 1962, Box 216.

${ }^{33}$ Vgl. Talking Papers, encl. to JCS Memo, 29.3.62, FRUS 61-63, XVII, S. 556-63; Nitze Memo, 21.3.62, ,Iran 1962 111---333،, OASD/ISA Files: General and Country, 1962, Box 89, RG 330, NACP; den erwarteten Beschwerden des Schahs über die Benachteiligung Irans im Vergleich zu Pakistan und der Türkei begegnete man dagegen sehr offensiv, unter anderem auch mit mehreren Hinweisen auf die Tatsache, dass die Iraner sich bislang nicht in der Lage gezeigt hatten, das gelieferte Material entsprechend instand zu halten. Vgl. MemCon, 12.4.62, FRUS 61-63, XVII, S. 598-604; State Briefing Paper, ,Comparison of MAP to Iran, Turkey and Pakistan', 3.4.62, ,Iran Subjects [...] VI (A)‘, NSF, Countries, Box 117, JFKL; vgl. auch JCS History Iran, S. 69; beispielsweise waren nur die Hälfte der iranischen F-86-Kampfflugzeuge aufgrund von Instandhaltungsproblemen einsatzfähig. Vgl. Wheeler Memo, 8.5.62; Blaisdell Memo, 17.12.62, ,Iran 1962 000.1---092‘, OASD/ISA Files: General and Country, 1962, Box 89, RG 330, NACP.

${ }^{34}$ Wie von Bill Bundy vorgeschlagen gewährte man nun vier statt nur zwei Staffeln F-5s, strich dafür im Gegenzug die Fregatten für die iranische Marine. Allerdings konnte der Schah 12 zusätzliche, allerdings veraltete C-47Transportflugzeuge durchsetzen. Vgl. MemCon, 12.4.62; MemCon, 12.4.62; MemCon, 13.4.62, FRUS 61-63, XVII, S. 590-8, 598-604, 605f.; Komer Memo, 12.4.62, ,Iran, Security 1961-1963‘, POF, Countries, Box 119, JFKL; vgl. auch JCS History Iran, S. 69f.; William Bundy reinterpretierte später die Rüstungsauflagen als Teil des vonseiten Kennedys erzwungenen Reformprogramms, Ausdruck der bald einsetzenden Selbstwahrnehmung der erfolgreichen reformistischen Intervention. Vgl. W. BUNDY, Tangled Web, S. 134f.; zur angeblichen Demütigung des Schahs in Gesprächen mit McNamara vgl. ebd., S. 560 n123; die Quellen stützen diese Behauptung nicht.

${ }^{35}$ Holmes Letter, 26.4.62, ,I-C. Correspondence with the Ambassador, 1962‘, NEA, GTI: RIAD, 1958-63, Box 5, vgl. oben S. 280 und 295f.; tatsächlich hatte der Anteil der noch unter Eisenhower eingeleiteten Maßnahmen zum Aufbau der Infrastruktur am MAP-Programm aufgrund der Fertigstellung der meisten Arbeiten inzwischen stark abgenommen. Somit war der Anteil militärischer Ausrüstung prozentual angestiegen. Vgl. State Briefing Paper, 3.4.62, ,Iran [...] Tab VI (8)‘, NSF, Countries, Box 117, JFKL.
} 
amerikanische Interesse an einer Fortsetzung der Reformpolitik betonte. Entgegen des Rates von Julius Holmes beglückwünschte er den Monarchen zu seinem ,exzellenten Premier“ und widersprach ihm zudem scharf, als dieser die Existenz einer starken Armee als Voraussetzung für die Umwandlung Irans in einen ,showcase“ für die Sache des Westens bezeichnete. Die Protokolle der Treffen zwischen Kennedy und dem iranischen Monarchen lassen durchaus eine kaum verhohlene Animosität zwischen beiden durchblicken. ${ }^{36}$ Im institutionellen Gedächtnis des State Department erinnerte man sich noch 1971: „There was never much love lost between he ruling Pahlavi dynasty and the two late Kennedys in those days." Der Schah selbst bezeichnete später die Jahre 1961 und 1962 als die „,schlimmste Phase“ in den amerikanischiranischen Beziehungen und beschwerte sich verbittert über die Versuche der ,great American liberals“, anderen ihre Vorstellungen von Demokratie aufzuzwingen. ${ }^{37}$

Trotz der persönlichen Missstimmungen war der Besuch aus Sicht Mohammad Rezās zweifellos ein großer Erfolg. So hatte Präsident Kennedy den Schah als „Schlüsselstein“ für den zukünftigen Erfolg in Iran bezeichnet. Außenminister 'Abbās Ārām resümierte im Anschluss an den Besuch erleichtert, das bestehende Regime werde nicht in Frage gestellt. ${ }^{38}$ Bob Komer glaubte ebenfalls, dass man die beiden zentralen US-Zielsetzungen - ,we achieved our objectives of reassuring the Shah and encouraging him to move forward on development and reforms“ - erreicht habe. ${ }^{39}$ Tatsächlich aber war man weit über das Ziel hinaus geschossen. Mohammad Rezā sabotierte auch im Anschluss an seinen USA-Besuch die Reformpolitik Aminis. Ermuntert vom „Erfolg“ seiner Gespräche in Washington, widersetzte er sich notwendigen Einsparungen am Militäretat und verweigerte dem Premier gleichzeitig jedwede Unterstützung dabei, andere Ressorts zum Maßhalten zu bewegen. ${ }^{40}$ Während die offenkundig vom Schah inspirierte Korruptionsuntersuchung gegen Mitarbeiter der Planorganisation die Wirtschaftsreformen der Regierung paralysierten, bekämpften die monarchischen Kräfte zugleich erfolgreich die Anti-Korruptionskampagne Aminis. ${ }^{41}$ Trotz der Verhaftungen vom

\footnotetext{
${ }^{36} \mathrm{Vgl}$. MemCon, 13.4.62, FRUS 61-63, XVII, S. 606-10.

${ }^{37}$ State \#115232, 25.6.71, POL Iran-US, CF 70-73, RG 59, NACP; die Beschwerde des Schahs aus dem Jahr 1969 zu finden in BILL, Eagle and Lion, S. 137; auch Bob Komer erinnerte sich an Kennedys Vorbehalte. „I don't think Kennedy thought much of the guy. Went around in these fancy uniforms. I don't think he was much impressed with the correspondence."; Robert W. Komer OHI, 11.8.87, OHIC, S. I-19; lange ging auch das Gerücht um, Kennedy habe den Schah in seiner Senatorenzeit öffentlich einen ,tin pot Dictator“ genannt. Vgl. Jack C. Miklos OHI, 28.7.86, ebd., S. I-52.

${ }^{38}$ Der Schah hatte gegenüber Kennedy betont, dass er „,not by nature a dictator“ sei, aber die Rückständigkeit Irans des Öfteren auch extralegale Methoden unumgänglich mache. Kennedy pflichtete insofern bei, als er betonte, dass in jedem Land die spezifisschen Umstände zu berücksichtigen seien. Vgl. MemCon, 13.4.62, FRUS 61-63, XVII, S. 609; der Schah äußerte sich im Anschluss an den Besuch zufrieden mit dessen Verlauf. Vgl. CIA Information Report, 13.5.62, ,Iran General 3/27/62-5/21/62‘, NSF, Countries, Box 116, JFKL.

${ }^{39}$ Komer Memo, 18.4.62, ,Iran Subjects, [...] 5/14/62', NSF, Countries, Box 117, JFKL.

${ }^{40}$ Gegenüber dem britischen Botschafter Harrison machte der Schah seine Position unmissverständlich klar. Nachdem der Leiter von ARMISH-MAAG ihn informiert hatte, dass seine Vorstellungen über eine $60 \%$-ige Budgetsteigerung des Militäretats unrealistisch seien, beschwerte er sich bei Harrison über die Haltung der Amerikaner. Vgl. Harrison to FO, No. 220, 5.3.62, PREM 11/3845, TNA:PRO; Cottam zufolge hatte der Schah die USA in erster Linie in der Absicht besucht, die Reaktion der Kennedy-Administration auf eine Ablösung Aminis zu sondieren. Er deutet dabei ein Nachgeben der US-Administration an, die trotz aller Bewunderung für Aminis Leistungen die monarchische Prärogative in der Frage des Regierungschefs akzeptierten. Vgl. COTTAM, Iran and the United States, S. $126 \mathrm{f}$.

${ }^{41}$ Ende Mai traten der Leiter der Planorganisation, Sāfi Asfijā, und sein Stellvertreter, Xodādād Farmānfarmāe ijān, zurück - die meisten anderen Mitarbeiter kündigten ebenfalls ihren baldigen Abschied an. Mit Ausnahme
} 
Januar 1962 blieb zudem die konservative Opposition sichtbar und einige Anzeichen deuteten auf eine versteckte Unterstützung seitens des Hofs hin. ${ }^{42}$ Nur verspätet reagierte man in Washington auf die aufziehende Krise in Iran, die das Überleben der Amini-Regierung in Frage stellte. Hauptaugenmerk lag zunächst auf der Umsetzung der während des Schah-Besuchs vereinbarten MAP-Beschlüsse. Wie nach allen vorherigen USA-Besuchen, versuchte Mohammad Rezā die seitens der US-Administration zugesagte materielle Unterstützung im Nachhinein nach oben zu revidieren. ${ }^{43}$ Iran Desk Officer Bowling warnte, dass sich die AminiRegierung angesichts des absehbaren gewaltigen Haushaltsdefizits - bereits im Vorfeld hatte die Kennedy-Administration die Fortsetzung direkter Budgethilfe grundsätzlich ausgeschlossen - zwischen einigen ihrer Projekte entscheiden müsse. Da die vereinbarte Absenkung der Sollstärke der Armee erst in der Zukunft für Einsparungen sorgen würde, müssten kurzfristig entweder der Dritte Plan, die bereits beschlossenen Lohnerhöhungen für Staatsdiener oder die Umsetzung der Landreform Arsanğānis ausgesetzt werden. Keiner dieser Schritte, so Bowling, würde für die Amini-Regierung folgenlos bleiben. Gleichzeitig warnte er, die Möglichkeiten der US-Administration in der nun entstandenen Lage seien nur begrenzt. ${ }^{44}$

Farmānfarmāèjāns konnte Amini die Planer überzeugen, ihre Arbeit fortzusetzen. Dennoch interpretierte die Botschaft die Vorgänge als ,expressive of the growing disillusionment of the more modern element in the government and society with Amini.“; Tehran \#548, 2.6.62, CF 60-63, 788.13/6-262; vgl. Xodādād Farmānfarmāeijān OHI, 17.12.82, IOHP; vgl. Eintrag vom 14.9.62, in: LILIENTHAL, Journals: V, S. 392ff.; angesichts seiner öffentlichen Selbstverpflichtung auf den Kampf gegen die Korruption fiel es Amini schwer, die offensichtlich politisch motivierte Kampagne gegen die Planorganisation und Ebtehāğ zu unterbinden. Zwischen April und Juni wurden die wichtigsten der zuvor angeklagten Offiziere auf freien Fuß gesetzt, bald darauf wurde schließlich auch Ebtehāğ entlassen, was ein quid pro quo nahe legt. Vgl. Tehran \#326, 13.1.62, 788.00/1-1362; Tehran \#A-297, 4.6.62, 788.00/6-462, CF 60-63; Battle Memo, 9.4.62, ,Iran General 3/27/62-5/21/62‘; Tehran \#983, 31.5.62, ,Iran General 5/22/62-6/30/62‘, NSF, Countries, Box 116, JFKL; MemCon, 31.5.62; Walsh Letter, 26.5.62, ,6. Plan Organization, 1962‘, NEA, GTI: RIAD, 1958-63, Box 6; bereits im Februar hatte der britische Botschafter über den Prestigeverlust Aminis berichtet. Er werde weithin als ein „, an American nominee“ betrachtet und das Ansehen der USA habe infolgedessen merklich gelitten: „A by-product of recent development is thus a significant increase in unpopularity for the Americans, who are held indirectly responsible by all those whose vested interests and privileges are under attack."; Harrison to FO, No. 9, 21.2.62, PREM 11/3846, TNA:PRO.

${ }^{42}$ Ein Vertrauter Aminis hatte bereits zuvor die Teheraner Botschaft informiert, Asadollah Rašidijān besuche nahezu täglich den Hof. Der Schah selbst gab gegenüber US-Offiziellen vor, er lehne die Aktivitäten dieser Gruppe streng ab. Vgl. Tehran \#368, 8.2.62, 788.00/2-862; Tehran \#293, 20.12.61, 788.11/12-2061, CF 60-63; CIA Information Report, 18.7.62, ,Iran General 7/18/62-7/23/62‘, NSF, Countries, Box 116, JFKL.

${ }_{43}$ Bundy berichtete dem Präsidenten, der Schah habe sein Verlangen nach Ausrüstung, ,that make no sense whatever in military terms“, nicht aufgegeben. Bundy Memo, 21.5.62, ,Military Assistance Programs, Section II“, NSF, Subjects, Box 298, JFKL; der letztlich vereinbarte Umfang des MAP-Fünfjahresplanes in ,Summary of Planned Deliveries of Military Equipment to Iran', encl. to Rusk Aide-Mémoire to Shah, 13.4.62, FRUS 61-63, XVII, S. 623ff.; zur genauen Ausrichtung der zukünftigen iranischen Streitkräfte wurde die Entsendung eines militärischen Planungsteams der JCS nach Iran vereinbart. Vgl. ebd., S. 659 Anm. o.N.; Mohammad Rezā berief sich nach einer Rückkehr nach Teheran auf angebliche persönliche Zusagen Kennedys, beim Aufbau einer 2.000 Mann starken „Kommandobrigade“ für Straßenschlachten behilflich zu sein. Zudem habe der Präsident die Lieferung eines $500 \mathrm{~kW}$ starken Senders zugesagt. Kennedy konnte sich an keine der angeblichen Zusagen erinnern. Komer informierte Anfang Juni, dass der Schah ,, is making noises about much bigger MAP package than we offered"; Komer Memo, 1.6.62, ebd., S. 700; Holmes unterstützte die Forderung nach einer Spezialeinheit, die in Zukunft den Einsatz der Fallschirmjäger vermeiden helfen und Unruhen unterhalb der ,kritischen“ Schwelle halten könne. Vgl. Brubeck Memo, 2.6.62; Komer Memo, 4.6.62, ,Iran General 8/12/62-8/31/62‘; Tehran \#1050, 26.6.62, ,Iran General 5/22/62-6/30/62‘, NSF, Countries, Box 116, JFKL.

${ }_{44}$ Bowling warnte: ,[...] we could not with impunity go too far in acting as a general comptroller of Iranian government expenditures. There is a little thing called national sovereignty."; Bowling Memo, 5.6.62, , Office Memoranda, 1962‘, Box 4; wahrscheinlich war es auch Bowling, der eine frühere Stellungnahme der Botschaft zu den Rücktritten in der Planorganisation inklusive Empfehlungen,,to keep the planners and the populace quiet" mit dem ironischen handschriftlichen Kommentar versehen hatte: „Are these one and the same?"; Walsh Letter, 31.5.62, ,6. Plan Organization, 1962', NEA, GTI: RIAD, 1958-63, Box 6; der Schah hatte während seines Besuchs einen letzten vergeblichen Versuch unternommen, weitere Budgethilfe durchzusetzen. Vgl. MemCon, 12.4.62, FRUS 61-63, XVII, S. 590-8. 
Im Juni 1962 berichtete die US-Botschaft in Teheran besorgt über die immer weiter eskalierende Haushaltskrise in Iran. Trotz absehbarer geringerer Einnahmen - neben der reduzierten US-Hilfe hatte das wirtschaftliche Stabilisierungsprogramm eine Rezession ausgelöst, mit direkten negativen Auswirkungen auf die Einnahmesituation - waren die geplanten Ausgaben rasant angewachsen. Angesichts der konstatierten Führungsschwäche Aminis in finanziellen Fragen unterstellte man seitens der Kennedy-Administration - wohl nicht ganz zu Unrecht -, dass der Ministerpräsident im Falle einer Krise auf die erneute Gewährung amerikanischer Soforthilfe spekuliere. ${ }^{45}$ Kaum war eine erneute Krisensituation in Teheran wahrnehmbar, zerfiel die seit Jahresbeginn 1962 erreichte Einigkeit in der Iranpolitik zwischen Weißen Haus und State Department wieder. Nachdem der Iran-Veteran Edward Mason mit einer äußerst pessimistischen Einschätzung von einem Trip nach Teheran zurückgekehrt war, empörte sich Bob Komer über die passive Reaktion seitens des State Department und AID. Aus Sicht des NSC-Stabs war in erster Linie der Schah durch seine Weigerung, den Militäretat zu kürzen, für die Krise verantwortlich. Botschaft und GTI allerdings widersprachen - der Minister-Counselor der Botschaft verstieg sich sogar auf die Behauptung, dass es , ,[...] probably be too much to expect Shah to bail Amini out of a situation essentially created by Amini himself". ${ }^{46}$ Das Weiße Haus reagierte verärgerte über diese Einstellung der Teheraner Botschaft und forderte stattdessen Holmes auf, umgehend beim Schah zu intervenieren, um dessen Unterstützung für Aminis Bemühungen zur Lösung der Finanzkrise einzufordern. ${ }^{47}$ Der Schah erweckte zwar in den darauffolgenden Wochen tatsächlich den Eindruck, bei der Lösung der Budgetkrise mitzuwirken - dennoch hatte die Amini-Regierung im Juni 1962 große Schwierigkeiten, die finanzielle Lage unter Kontrolle zu bekommen. Gleichzeitig ersuchte Amini vergeblich - unterstützt von einer dringlichen Anfrage seitens des pro-amerikanischen SAWAK-Direktors Pākrawān - eine Zusage der USAdministration für die erneute Gewährung einer direkten Budgethilfe zu erlangen. Sowohl die Botschaft in Teheran wie auch State Department und Weißes Haus waren jedoch in dieser Frage unerbittlich. „All of us here believe Iran should be forced to dig itself out of this one instead of our bailing it out again“, kommentierte Komer. ${ }^{48}$

\footnotetext{
${ }^{45}$ Vgl. Tehran \#1008, 12.6.62, FRUS 61-63, XVII, S. 720-3; in diesem Telegramm veranschlagte die Botschaft das derzeit absehbare Defizit auf die erstaunliche Summe von \$250 Millionen. Im Juni war bereits von einem „horrendous deficit“" die Rede. Walsh Letter, 5.6.62, ,7. Iran Country Budget, 1962‘, NEA, GTI: RIAD, 1958-63, Box 7; das Country Team sollte feststellen, ob Amini ,changed objective of fiscal responsibility to one of building case for massive U.S. budgetary assistance?"; State \#894, 1.6.62, ,Iran General 5/22/62-6/30/62“, NSF, Countries, Box 116, JFKL.

${ }^{46}$ Das Zitat des Minister-Counselor Stuart Rockwell in Tehran Telegram \#1008 to State, 12.6.62, FRUS 61-63, XVII, 720-3, hier 722; Edward Mason kehrte aus Iran zurück mit einem ,gloomy report“: Amini sei mit seinen Kräften am Ende und habe sein Kabinett nicht mehr unter Kontrolle. Trotz drohenden Defizits von ca. \$170 Millionen weigere sich der Schah Unterstützung zu leisten. Vgl. Komer Memo, 15.6.62, ,Staff Memoranda, Robert Komer 6/62', NSF, M\&M, Box 322; Tehran \#953, 23.5.62; State \#922, 16.6.62, ,Iran General 5/22/62-6/30/62‘, NSF, Countries, Box 116, JFKL.

47 Vgl. State \#921, 15.6.62, FRUS 61-63, XVII, S. 726f.; Komer versuchte vergeblich, eine direkte Nachricht Kennedys an den Schah bezüglich der Notwendigkeit einer schnellen Lösung der Krise (,,a bit of top level preventive medicine“) durchzusetzen. Vgl. State \#928, 19.6.62; Komer Memo, 20.6.62, , Iran General 5/22/626/30/62', NSF, Countries, Box 116; Draft Personal Message, o.D., encl. to Komer Memo, 18.6.62, ,Iran, 1961 [...] Memoranda', NSF, RWK, Box 424, JFKL.

${ }^{48}$ Komer Memo, 16.7.62, FRUS 61-63, XVIII, S. 9f.; Vgl. Tehran \#1041, 24.6.62, ebd., FRUS 61-63, XVII, S. 748-51; Pākrawān hatte einen CIA-Mitarbeiter direkt informiert, dass Amini „, needed $\$ 80$ million for civilian
} 
'Ali Amini gelang es dennoch nicht, gegenüber seinem Kabinett und den verschiedenen politischen Machtzentren in Iran die notwendigen Kürzungen durchzusetzen. Vor Ort war Holmes über die Schwierigkeiten des Ministerpräsidenten gut informiert, gab sich gegenüber Washington dennoch zuversichtlich, dass es außer Amini niemanden gäbe, der die Krise meistern könne. Zugleich weigerte sich Holmes, den Schah zu direkten Entscheidungen über Budgetkürzungen $\mathrm{zu}$ bewegen, dabei etwas fragwürdig argumentierend, die bisherige USPolitik habe darauf abgezielt, den Schah aus den alltäglichen Regierungsentscheidungen herauszuhalten. Würde man nun, so Holmes, bewusst eine Intervention Mohammad Rezās in konkreten Haushaltsentscheidungen auslösen, wäre das gesamte Amini-Experiment in Frage gestellt - der Schah würde zwangsläufig wieder die direkte Führung der Regierungsgeschäfte übernehmen und Amini würde ein zweiter „Eg̈bāl““ ${ }^{49}$ Hier zeigte sich, wie stark die vorherigen Kontoversen über die Task Force-Politik auch weiterhin auf die US-Politik einwirkten. Ohne Aussicht auf amerikanische Unterstützung und unfähig, die notwendigen Kürzungen durchzusetzen, trat Amini schließlich am 17. Juli 1962 zurück. Im Nachhinein entstand eine Kontroverse über den Ablauf der Ereignisse, die zur Resignation Aminis geführt hatten. Bob Komer stellte später eine detaillierte Liste der verschiedenen Berichte der Botschaft und der Instruktionen seitens des Weißen Hauses und des State Department während der Krise zusammen. Kennedy konnte sich aber offenbar nicht für eine Reaktion entscheiden, mit Bezug auf ,what is obviously failure of Holmes to carry out his instructions [...]. “50 In GTI bestritt man allerdings die Vorwürfe gegen Holmes und betonte dagegen, es sei ,inescapable that we could have saved Amini with budgetary support, the earlier the better." Die Grundsatzentscheidung der Kennedy-Administration gegen weitere Budgetzuschüsse habe letzten Endes das Schicksal der Amini-Regierung besiegelt. Es sei ohnehin nicht absehbar gewesen, dass der Ministerpräsident so unvermittelt seinen Rücktritt einreichen würde, so die spätere Einschätzung. In der Tat glaubten nur die wenigsten Beobachter vor Ort, dass Amini von alleine sein Amt aufgeben könnte, wenn auch einige seiner Gesprächspartner die physische und psychische Erschöpfung des Ministerpräsidenten registriert hatten. ${ }^{51}$ Andererseits war die Empörung der Komer-Hansen-Gruppe im Weißen Haus durchaus nachvollziehbar. Zweifellos hatte der US-Botschafter vor Ort durch seine Weigerung, eine Lösung der Haushaltskrise durch direktes Eingreifen des Schahs zu erreichen, den Niedergang der Reformregierung

budget and $\$ 20$ million for military budget in order to survive."; vgl. State \#33, 13.7.62, 888.10/7-1162; Tehran \#76, 18.7.62, 888.10/7-1862, CF 60-63.

${ }_{49}$ Vgl. State \#921, 15.6.62, FRUS 61-63, XVII, S. 726f.; indem Holmes Druck auf den Schah mit dem Ziel, Budgetkürzungen durchzusetzen, mit einer Aufgabe des Amini-Experiments gleichsetzte, entzog er sich den Forderungen aus Washington nach weiterreichenden Maßnahmen. Vgl. Tehran \#15, 4.7.62, , Iran General 7/1/627/17/62،, NSF, Countries, Box 116, JFKL.

${ }^{50}$ Kaysen Memo, 5.10.62, ,Staff Memoranda, Carl Kaysen, 8/62-12/62‘, NSF, M\&M, Box 320; das Country Team hatte das Szenario einer Amini-Entlassung bereits in einem Contingency Paper durchgespielt. Vgl. Rockwell Letter, 5.6.62, ,3-A/1. Military Contingency Planning‘, NEA, GTI: RIAD, 1958-63, Box 6.

${ }^{51}$ Draft Background Paper, o.D. [Juli 1962], ,I-A. History and Background, General', NEA, GTI: RIAD, 195863, Box 5; dieser wahrscheinlich von Bowling verfasste Entwurf war in erster Linie eine Verteidigung des Vorgehens der Botschaft während der Krise. Der Verfasser insistierte trotz Aminis Rückzug, dass ,[...] Ambassador's feeling that nothing would served by going to the Shah and dumping the problem in his lap still seems essentially sound." 
unumgänglich gemacht. ${ }^{52}$ Während man seitens NEA die Ansicht vertrat, der Schah habe alles in seiner Macht stehende unternommen, die Regierung zu unterstützen, kamen die Analysten der CIA zu einem anderen Ergebnis. Offenbar habe der Schah die Bemühungen Aminis nur begrenzt unterstützt und sei ,probably not unhappy to see him resign. “53

Nach dem Rücktritt des Amini-Kabinetts stand die im Kontext des Schah-Besuchs konzipierte US-Politik - Fortsetzung des Reformprogramms bei gleichzeitiger Rückversicherung des Schahs - vor einem Scherbenhaufen. Tatsächlich hatte sich die grundsätzliche Kritik der Komer-Hansen-Gruppe vom Herbst 1961 am eingeschlagenen Kurs als berechtigt erwiesen. Ohne politische Basis und angesichts der eingeleiteten Reformmaßnahmen mit zahlreichen innenpolitischen Gegnern konfrontiert, besaß Amini im Grunde nur geringe Chancen auf langfristiges politisches Überleben, zumal er letzten Endes auf die Hilfe des ihn nur vordergründig unterstützenden Schahs bzw. der amerikanischen Schutzmacht angewiesen war. Letztere war in der Zwischenzeit - ein von der jeder Intervention in die Innenpolitik gegenüber skeptisch eingestellten US-Botschaft vor Ort zusätzlich verstärkter Trend - weitaus weniger bereit als noch Mitte 1961, den notwendigen Druck auszuüben. Tatsächlich gab Amini in einer Pressekonferenz unmittelbar nach seinem Rücktritt der mangelnden Unterstützung durch die USA die Schuld an seinem Scheitern, wenngleich er diese Behauptung nach einigen Tagen wieder zurücknahm. ${ }^{54}$ Bob Komer drängte dennoch intern auf entschlossenes Handeln (,,an allout effort“) seitens der Administration mit dem Ziel, die Amini-Regierung zu retten: „If we return to more of the same in Iran, we'll face chaos there all too soon. “55 Die Administration konnte sich jedoch zu einem solchen Schritt nicht durchringen.

In Teheran hatte inzwischen Schah Mohammad Rezā eine Nachfolgeregierung bestellt. Aminis Nachfolger wurde Asadollah 'Alam, bislang Direktor der Pahlavi Foundation, zudem Jugendfreund und enger Vertrauter des Schahs. Botschafter Holmes schätzte ihn als ,devoted servant“ des Monarchen ein - seine Regierungsübernahme repräsentiere „the closest thing to

\footnotetext{
${ }^{52}$ Vgl. State \#47, 18.7.62, 888.10/7-1762, CF 60-63; Amini hatte allerdings gegenüber US-Offiziellen mehrfach die Höhe des Militärbudgets als einen der Hauptgründe für die Haushaltskrise genannt. Vgl. Tehran \#62, 16.7.62, $888.10 / 7-1662$, ebd.

53 CIA Memo Nr. 2412/62, 18.7.62, FRUS 61-63, XVII-XVIII-XX-XXI Suppl., Dok. 94; Holmes dagegen berichtete von entschlossener Unterstützung seitens des Schahs für die Anstrengungen Aminis, eine Einschätzung, die ex post facto auch von NEA unterstützt wurde. Vgl. Tehran \#45, 11.7.62, 888.10/7-1162, CF 60-63; Draft Background Paper, Anm. 51; Tehran \#A-47, 25.7.62, ,Iran, 1961-1962 (Folder 1 of 2)', NSF, RWK, Box 424, JFKL.

${ }^{54}$ Vgl. Draft Paper for Senate Foreign Relations Subcommittee, o.D., ,I-A. History and Background, General', NEA, GTI: RIAD, 1958-63, Box 5; zu Aminis öffentlichen Verlautbarungen und der US-Reaktion vgl. Tehran \#91, 19.7.62, ,Iran General 7/18/62-7/23/62‘, NSF, Countries, Box 116, JFKL; die Amini-Regierung war mit ca. \$87 Millionen unterstützt worden, wohingegen die durchschnittliche finanzielle Hilfe in den vier vorausgegangenen Jahren nur etwa \$59 Millionen betragen hatte. Vgl. FRUS 61-63, XVIII, S. 11, Anm. o.N.; S. 16, $n 1$; falsche Angaben bei BARRETT, Greater Middle East, S. 239.

${ }_{55}$ Komer Memo, 18.7.62, FRUS 61-63, XVIII, S. 11; in NEA kritisierte man die „,basic misapprehension here in Washington“, derzufolge ,if we just tell these client countries what they have to do, they will dutifully do it. These same great minds refuse to admit that if we have a stranglehold on a little country like Iran, it also has one on us."; Draft Background Paper, o.D. [Juli 1962], ,I-A. History and Background, General', NEA, GTI: RIAD, 1958-63, Box 5 .
} 
direct rule by the Shah." Der Schah selbst sei nun sein eigener Premier, sekundierte die CIA. ${ }^{56}$ Noch ein Jahr zuvor hatte eine NEA-Studie eine solche Konstellation als potentiell „desaströs“ bezeichnet. Nun arrangierte man sich notgedrungen mit der neuen Situation, blieb aber weiterhin ablehnend gegenüber einem Budgetzuschuss eingestellt. Tatsächlich versprachen sowohl der Schah wie auch 'Alam eine Fortsetzung des Reformprogramms. ${ }^{57}$ Es gelang dem neuen Finanzminister Behnijā im August 1962, das Budget zu stabilisieren, indem er die ambitionierten Programme des dritten Siebenjahresplanes auf das allernötigste reduzierte und weitere Ausgabenbeschränkungen durchsetzte - ein Erfolg, der offenbar in erster Linie der nun vorhandenen Unterstützung Mohammad Rezās zuzuschreiben war. Somit gelang es der 'AlamRegierung, das Defizit nochmals um \$28 Millionen zu reduzieren, womit die unmittelbare Haushaltskrise vorerst gelöst schien. ${ }^{58}$ Dennoch herrschte innerhalb der Administration eher Skepsis, was die mittelfristigen Aussichten für die 'Alam-Regierung anging. Eine Einschätzung der CIA gestand zwar ein, die eigenen Prognosen der letzten Jahre seien vielleicht zu pessimistisch gewesenen. Dennoch bedeute Aminis Demission letztlich die Rückkehr zu einer Situation ,very like that which existed before he came to office.“ Erneut sei Mohammad Rezā in einer Position, in der er persönlich für die politische wie wirtschaftliche Entwicklung des Landes verantwortlich sei. Damit sei nicht auszuschließen, dass sich die zerstrittene Opposition wie im Mai 1961 erneut gegen den monarchischen Absolutismus zusammenfinden könne, eine Möglichkeit, die durch die vornehmliche Beschäftigung des Schahs mit militärischen Angelegenheiten und das Desinteresse an finanziellen und administrativen Fragen noch wahrscheinlicher gemacht werde. Trotz der Zerstrittenheit der Opposition und der gewachsenen Effizienz des Sicherheitsapparates hielt man seitens der CIA daher erneut „Sturmwarnungen“ für angebracht. ${ }^{59}$

\footnotetext{
${ }^{56}$ Tehran \#86, 19.7.62, ,Iran General 7/8/62-7/23/62‘; CIA Memo, 26.7.62, ,Iran General 7/24/62-7/231/62‘, NSF, Countries, Box 116; der CIA zufolge hatte die Berufung sogar auf Vorschlag Aminis stattgefunden. Vgl. CIA Memo, 20.7.62, ,Iran 1961-1962 (Folder 1 of 2), NSF, RWK, Box 424, JFKL; ein US-Diplomat beschrieb 'Alam als ein "good example of an Iranian feudal landlord" wenngleich "more enlightened" als die meisten seiner Klassenangehörigen. Meshed \#49, 29.5.61, 788.00/5-2961, CF 60-63; ein Special National Intelligence Estimate vom September 1962 konstatierte nüchtern, die Berufung 'Alams bedeute, dass der Schah selbst ,intended to reassume direct responsibility for governing Iran."; CIA, SNIE 34-62, 7.9.62, FRUS 61-63, XVIII, S. 85; zu 'Alam vgl. MiLANI, Eminent Persians: I, S. 44-55; vgl. auch LADJEVARDI, Xāterāt-e Forugi i, S. 255-8; „,The Shah's right hand, sometimes his brain, often his backbone, never his conscience", so die treffliche Beschreibung eines Diplomaten. PRECHT, A Diplomat's Progress, S. 87.

${ }_{57}$ Vgl. oben S. 277, Anm. 167; vgl. Tehran \#90, 19.7.62; State \#58, 19.7.62, ,Iran General 7/18/62-7/23/62', NSF, Countries, Box 116, JFKL; wenig überraschend versuchte auch die neue Regierung, die USA von der Notwendigkeit sofortiger Finanzhilfen zu überzeugen, dieses Mal allerdings mit starker Unterstützung des Schahs. Vgl. Tehran \#110, 24.7.62, ebd.; Komer im Weißen Haus zeigte größere Bereitschaft für eine neue Finanzspritze in einer Größenordnung von etwa \$20-30 Millionen und appellierte gegen den ,wait and see“-Ansatz von Holmes, allerdings unter der Voraussetzung eines iranischen Verzichts auf Erhöhung des Militärbudgets. Vgl. Komer Memo, 19.7.62, FRUS 61-63, XVIII, S. 16; allerdings gab es auch innerhalb NEAs bald Überlegungen, der 'AlamRegierung mit Krediten in Höhe von ca. \$40 Millionen beizustehen im Falle einer „Lösung“ der Finanzkrise durch erhöhte Schuldenaufnahme und Kooperation mit Weltbank und Internationalem Währungsfonds. Vgl. Report, o.D., encl. to Miner Memo, 3.8.62; Talbot Letter, 7.8.62, ,I. The Prime Minister', NEA, GTI: RIAD, 1958-63, Box 5 .

${ }_{58}^{5} \mathrm{Vgl}$. Tehran \#222, 18.8.62; Tehran \#A-156, 20.8.62, ,Iran General 8/12/62-8/31/62‘, NSF, Countries, Box 116, JFKL; allerdings konnte auch 'Alam den Anstieg des Militäretats um 10,5\% nicht verhindern, wenn auch der Anstieg nur halb so hoch ausfiel wie noch im letzten (gescheiterten) Entwurf der Amini-Regierung.

${ }^{59}$ CIA Board of National Estimates Memo, 16.8.62, encl. to Komer Memo, 18.8.62, ,Iran, 1961-1962 (Folder 1 of 2)', NSF, RWK, Box 424, JFKL.
} 
Zumindest was die Priorisierung militärischer Belange durch den Schah anging, war der CIA kaum zu widersprechen. Während sich in Teheran die Haushaltskrise zugespitzt hatte und die Amini-Regierung verzweifelt um Etatkürzungen kämpfte, hatte sich der Schah in erster Linie mit den Details des während seines Besuchs in Washington festgelegten MAP-Programmes beschäftigt. Bald nach seiner Rückkehr gab es erste Anzeichen dafür, dass Mohammad Rezā größere Änderungen am Programm durchzusetzen gedachte. Wie in Washington vereinbart, traf im Mai 1962 ein Planning Team der JCS in Teheran ein, um die Details des MAPProgrammes, die Truppenreduktion und die den iranischen Streitkräften zugedachte Rolle in den globalen Planungen zu diskutieren. Wie zu erwarten, versuchte der Schah umgehend, grundsätzliche Revisionen am Programm durchzusetzen. Er verlangte nach mehr und leistungsfähigeren Kampfflugzeugen, 3.000 weiteren Transportfahrzeugen, einem modernen Radarsystem, einem zusätzlichen Fallschirmjägerregiment, der Restitution der während seines Staatsbesuchs aus dem MAP-Programm gestrichenen Fregatten und er versuchte zudem die Senkung der Sollstärke um 50.000 Mann zu verhindern. Sein Ziel sei es, informierte er die verblüfften US-Planer, die „beste Armee in diesem Teil der Welt aufzubauen.“60 Sollte noch irgendjemand Zweifel daran gehabt haben, dass die vor dem Staatsbesuch vereinbarte amerikanische Strategie gescheitert war, wurde er Anfang Juli durch einen Brief des Schahs an Präsident Kennedy eines Besseren belehrt. In seinem Schreiben betonte er die exponierte strategische Lage Irans und leitete daraus weitere militärische Notwendigkeiten ab, unter anderem die Umrüstung der iranischen Luftwaffe auf brandneue McDonnell F-4 PhantomJagdflugzeuge, mit deren Auslieferung an die US-Luftwaffe gerade erst begonnen worden war, was angesichts der strategischen Gesamtlage und des eigenen Ausbildungsstands als eine doch recht überambitionierte Intention zu werten ist. Zudem hatte er als „Alternative“ für die zwei Fregatten die Überlassung von zwei alten Zerstörern vorgeschlagen, vorgeblich um das durch sowjetische Lieferungen an Irak gestörte maritime Gleichgewicht im Persischen Golf wiederherzustellen. $^{61}$ Gegen die Empfehlung Bob Komers, der die Gelegenheit zur Bekräftigung des reformistischen Interventionismus des Vorjahres zu nutzen gedachte, blieb die Antwort des Präsidenten unbestimmt. Indirekt akzeptierte Kennedy die Rückkehr des Schahs in eine herausgehobene politische Position in Iran, wenn auch seine konkreten militärischen Forderungen dilatorisch behandelt wurden. ${ }^{62}$

\footnotetext{
60 Tehran \#944, 19.5.62, 788.5/5-1962, CF 60-63; der Schah präferierte F-104 Starfighter gegenüber den angebotenen $F-5$ und verlangte zudem sechs Staffeln statt der im Programm vorgesehenen vier, obwohl die USSeite gerade erst während seines Besuchs einer Verdopplung auf vier Staffeln zugestimmt hatte. Zudem verlangte er zwei zusätzliche Lockheed C-140-Transporter neben den zugesagten vier C-130. Vgl. State \#846, 15.5.62, 788.5/5-1562; Tehran \#16, 4.7.61, 788.00/7-461, ebd.; vgl. MemCon, 12.4.62, FRUS 61-63, XVII, S. 598-504, hier 601; ,Summary of Planned Deliveries', encl. to Rusk Aide-Mémoire, 13.4.62, ebd., S. 623ff.; vgl. auch JCS History Iran, S. 70f.

${ }_{61}$ Vgl. Shah Letter, 9.7.62, ,Iran General 7/24/61-7/31/62‘, NSF, Countries, Box 116, JFKL; Anfang Juli hatte sich der Schah gegenüber Holmes zuvor auf ein Minimum von 170.000 Mann festgelegt. Vgl. Tehran \#5, 1.7.62, 788.11/7-162, CF 60-63; Holmes Letter, 10.7.62, ,I-C. Corresp[...] 1962‘, NEA, GTI: RIAD, 1958-63, Box 5; in Washington hatte Mohammad Rezā die gestiegene Bedrohung durch den wachsenden Einflusses Nassers im Persischen Golf betont.

${ }^{62}$ Vgl. Kennedy Letter, 1.8.62, FRUS 61-63, XVIII, S. 21ff.; Komer schätzte die Mehrkosten im Falle einer Erfüllung der militärischen Wünsche auf \$100-135 Millionen zusätzlich. Vgl. Komer Memos, 12./27.7.62; ,Iran, 1961-1962, White House Memoranda', NSF, RWK, Box 424; Komer Memo, 18.7.62, ,Staff Memoranda, Robert Komer $7 / 62^{\circ}$, NSF, M\&M, Box 322, JFKL.
} 
Zwar hatte auch Kennedy selbst intern die Notwendigkeit betont, den „Napoleonic complex“ Mohammad Rezās zu überwinden und diesen endlich von der primären Notwendigkeit innerer Reformen $\mathrm{zu}$ überzeugen. ${ }^{63}$ Angesichts des Verlustes der pro-amerikanischen AminiRegierung und dem Mangel an politischen Alternativen im Sommer 1962 gab es allerdings nur wenige Befürworter einer Wiederaufnahme der politischen Interventionen. Im State Department sah man es sogar als zentrales Ziel an, eine Neuauflage der Iran Task Force zu verhindern. ${ }^{64}$ Statt eine neue Grundsatzdiskussion über die Ausrichtung der eigenen Iranpolitik zu führen, entschloss sich der Präsident, einen bereits im Vorfeld geplanten Besuch des Vizepräsidenten Lyndon B. Johnson in Iran im August 1962 zu einer weiteren Rückversicherung des Schahs zu benutzen. Johnson, vor der Wahl aus vorwiegend taktischen Gründen ins Ticket aufgenommen, hatte bis dato nur eine äußerst limitierte Rolle bei der Ausformulierung der US-Politik gespielt, eine Ausgrenzung, die in erster Linie für die persönlichen Animositäten zwischen ihm und den „Kennedy-Leuten“ und insbesondere für sein zerrüttetes persönliches Verhältnis mit dem Präsidentenbruder Robert Kennedy verantwortlich gewesen war. Zwar wurde Johnson noch vor dem Antritt seiner Reise, die ihn neben Iran auch noch in die Türkei und eine Reihe anderer Anrainerstaaten des Mittelmeers führte, instruiert, den Schah auch mit Nachdruck auf die Fortsetzung der inneren Reformen hinzuweisen. ${ }^{65}$ Tatsächlich bewerteten die meisten Beobachter den Besuch des Vizepräsidenten im Anschluss als Erfolg. Lyndon Johnson hielt sich in seinen Gesprächen mit dem Schah und Ministerpräsident 'Alam eng an seine Instruktionen, innere Reformen anmahnend und Diskussionen über das MAP-Paket ausweichend begegnend. Zugleich hatte er sich durch sein Auftreten als hemdsärmeliger und offener Texaner durchaus Sympathien in der iranischen Öffentlichkeit erworben, während die iranischen Eliten, so berichtete Holmes im Anschluss aus Teheran, Johnsons Kontaktversuche zur Bevölkerung eher mit Erstaunen und Verwunderung begegneten. ${ }^{66}$ Nach seiner Rückkehr berichtete Johnson dem Präsidenten, dass man den Schah,

\footnotetext{
${ }^{63}$ Komer erwähnte diese Bemerkung des Präsidenten in einem Memorandum an den Chief der Near East and South Asia Division der CIA, James H. Critchfield. Man solle die Aufmerksamkeit des Schahs endlich lenken „onto the things that will make or break the house of Pahlavi.“; Anlass war ein Bericht des Veteranen der politischen Kriegführung aus der Eisenhower-Zeit, C.D. Jackson, über einen Besuch in Teheran, in dem dieser von verbitterten Beschwerden über die nicht ausreichende Militärhilfe seitens der USA berichtet hatte. Vgl. Jackson Report, 7.8.62; Komer Memo to Critchfield, 21.8.62, ,Iran, 1961-1962 [White House Memoranda]', NSF, RWK, Box 424, JFKL.

${ }^{64}$ Vgl. Minor Memo to Talbot, 10.7.62, I. The Shah \& Court (and Govt) 1962‘, NEA, GTI: RIAD, 1958-63, Box 4, RG 59, NACP.

${ }^{65}$ Zum belasteten Verhältnis zu Robert Kennedy vgl. SHeSOL, Mutual Contempt; Komer hatte den Präsidenten im Vorfeld ermahnt, Johnson befürchte wieder einmal auf eine politisch zweitrangige Goodwill-Tour entsandt zu werden und empfahl die Betonung der Wichtigkeit der Reise, u.a. eine Bekräftigung der unveränderten USUnterstützung für Iran. Zu Johnsons elf Auslandsreisen in 33 Länder als Vizepräsident vgl. ebd., S. 88ff.; LERNER, Vice Presidential Travels; zur Iranreise vgl. Komer Memo, 20.8.62, ,V.P. Trip [...] 5/62-8/62‘, NSF, Trips and Conferences, Box 243; Komer Memo, ,Staff Memoranda: Robert Komer, 8/62, NSF, M\&M, Box 322, JFKL.

${ }^{66}$ Vgl. Tehran \#284 und \#285, 26.8.62, FRUS 61-63, XVII-XVIII-XX-XXI Suppl., Dok. 97 u. 98 ; vgl. z.B. Johnsons Rede vor der Iran-America Society in Incoming Ankara \#261, 27.8.62, ,VP [...] 1962, [2of3]', Vice Presidential Papers, Vice Presidential Security File, Box 2, LBJL; ehemalige Botschaftsangehörige erinnerten sich später an den Johnson-Besuch als ,,spektakuläres“ Ereignis. Seine Auftritte prägten später den Ausdruck, „JohnsonStil" für besonders publikumsnahe Wahlkampfauftritte. Johnson reiste immer mit einer großen Entourage inklusive Masseur und neigte neben spontanen Kontakten zur Bevölkerung zudem zu exzentrischen Aktionen. So bestand er z.B. für seine Übernachtungen in einem historischen Palast auf den sofortigen Einbau einer modernen Klimaanlage trotz der einhergehenden Beschädigungen der Bausubstanz. Vgl. William Green Miller OHI,
} 
trotz seiner Defizite, als „valuable asset“ akzeptieren müsse, ,since we have no acceptable alternative. “67 Selbst die CIA konstatierte etwas resigniert, die Ansicht des Schahs, Iran sei noch nicht bereit für eine ,true parliamentary government“, sei nicht ganz von der Hand zu weisen. Offenbar sah man auch hier keine Alternativen zum Schah, bewertete aber die politischen Strukturen in Iran weiterhin als ,,zerbrechlich“. 68

Die Auseinandersetzung zwischen Befürwortern und Gegnern reformistischer Intervention war auch während des Johnson-Besuchs in Iran fortgeführt worden. Offenbar als Ausgleich für die fast widerstandslose Aufgabe Aminis durfte Bob Komer den Vizepräsidenten auf seiner Reise begleiten. Obwohl Komer während seines Aufenthalts in Iran durchaus einige kritische Stimmen sammelte und insgesamt auf stärkere Betonung des Reformgedankens drängte, wurden die Hoffnungen seiner Unterstützer in Washington nicht erfüllt. Holmes und Yatsevitch schränkten Komers Handlungsspielraum in Teheran erfolgreich ein - zudem schien Komer selbst für die vielen Anreize der kaiserlichen Gesellschaft durchaus anfällig. ${ }^{69}$ Die entschlossensten Gegner des Schahs in Washington aber verstärkten nun den Druck auf die Administration, um endlich eine politische Kehrtwende in Teheran einzuleiten, allen voran der eng mit der Kennedy-Familie verbundene Supreme Court-Richter William O. Douglas. In seinen Memoiren behauptete Douglas später, seine fortwährende Lobbyarbeit gegen den Schah habe nach dem Washington-Besuch endlich Früchte getragen. Präsident Kennedy habe schließlich seine Überzeugung geteilt, dass Mohammad Rezā korrupt und auch unglaubwürdig sei - tatsächlich habe Kennedy sogar Pläne zur erzwungenen Abdankung des Schahs und die Einsetzung einer eher mit repräsentativen Funktionen ausgestatteten Regentschaft gebilligt, einhergehend mit einer durch amerikanische Intervention oktroyierten Parlamentarisierung Irans. Wenig überraschend ist, dass Douglas“ Behauptungen recht unkritisch von der Forschung aufgenommen wurden. ${ }^{70}$ Tatsächlich hatte Douglas während des Besuchs des Vizepräsidenten in Teheran angerufen und Bob Komer über seine Regentschaftspläne

10.2.2003; Maurice Williams OHI, 15.5.96, FAOH; für eine generelle Einschätzung der Reisediplomatie Johnsons als erfolgreiche ,cultural diplomacy“ vgl. LERNER, Vice Presidential Travels; der Iranbesuch von 1962, wenn auch mit zahlreichen Fehlinformationen, in ebd., S. 364-70.

${ }^{67}$ Zit. aus ed. note in FRUS 1961-63, XVIII, S. 72; ,Points for Oral Report to President', o.D., ,Vice President's Trip, 8/22/62-9/8/62', Robert W. Komer Papers', NSF, RWK, Box 431; Ankara \#258, 27.8.62, , V.P. Trip [...] 9/6/62', NSF, Trips and Conferences, Box 243, JFKL; eine handschriftliche Version von Johnsons Bericht enthält ebenfalls das wiedergegebene Zitat, zugleich aber auch eine amateurhafte und holzschnittartige Betrachtung über die geopolitische Gesamtlage der Nahostregion. Vgl. Draft Report, o.D., ,VP Johnson's [...] 1962, [1 of 3]", Vice Presidential Papers, Vice Presidential Security File, Box 2, LBJL; Johnson begann in der Folgezeit eine persönliche Korrespondenz mit dem Monarchen, die späteren engen persönlichen Bindungen vorwegnehmend. Vgl. Shah Letter, 10.11.62., ,Iran, 1961-1962 [Folder 1 of 2]', NSF, RWK, Box 424, JFKL; vgl. auch Scoresheet, ,Vice-Presidential Promises‘, 13.12.62, ,Office Memoranda, 1962‘, NEA, GTI: RIAD, 1958-63, Box 4; zum Johnson-Besuch vgl. auch BILL, Eagle and Lion, S. 140f.

${ }^{68}$ Die Nachrichtendienste rechneten zwar mit sich bald wieder erhöhendem öffentlichem Druck auf den Schah, gaben sich andererseits überzeugt, Mohammad Rezā würde nicht mehr bereit sein, seine Prärogative aufzugeben. Insgesamt war die Prognose optimistischer als frühere Analysen, auch wenn man an der Unabänderlichkeit von ,profound political and social change“ festhielt; CIA, SNIE 34-62, 7.9.62, FRUS 61-63, XVIII, S. 84-8.

${ }_{69}$ Vgl. MemCon, 25.8.62, , Vice [...] -9/8/62', Robert W. Komer Papers', NSF, RWK, Box 431; Komer Memo for the Record, 11.10.62, ,Staff [...]-10/62‘, NSF, M\&M, Box 322, JFKL; Yatsevitch hatte von Holmes den Auftrag erhalten, Komer unmittelbar nach dessen Ankunft mit Ausflügen in die Provinzen zu unterhalten, um diesen möglichst weit vom Hof fernzuhalten. Vgl. Gratian Yatsevitch OHI, 5.11.88, OHIC; S. I-98; Robert W. Komer OHI, 11.8.87, OHIC, S. I-27ff.

${ }^{70}$ Vgl. Douglas, Court Years, S. 303f.; zur Wirkung auf die Forschung vgl. z.B. BiLl, Eagle and Lion, S. 137. 
informiert. Dabei beschwerte er sich, wie Komer anschließend zu Protokoll gab, die USA könnten es sich nicht leisten, in der heutigen Zeit absolute Monarchien zu unterstützen. Mohammad Rezās Reform-Rhetorik sei nur geschickte „Public Relations“, seine Landreform ein Täuschungsmanöver - zudem habe er \$400 Millionen auf Bankkonten in der Schweiz und Israel beiseite geschafft. Zugleich beschwerte Douglas sich mit Nachdruck über die Unterwürfigkeit der amerikanischen Vertreter vor Ort und die angeblich dominierende Rolle der CIA in dem Land. Douglas konstatierte: „The only solution to the Persian situation is to get rid of the Shah." Mit dieser weitgehenden Forderung ging er allerdings sogar dem ansonsten durchaus Schah-kritisch eingestellten Bob Komer viel zu weit. Douglas stünde unter dem Einfluss verbitterter iranischer Regimegegner, die zudem kaum in der Lage seien, Iran einen bessere Führung zu geben als das gegenwärtige Regime. ${ }^{71}$ Radikale Vorschläge à la Douglas fanden tatsächlich kaum noch Beachtung. Selbst die alte Komer-Hansen-Gruppe war nun uneinig und infolgedessen noch weniger in der Lage, die eingeschlagene Richtung der Iranpolitik nachhaltig zu beeinflussen. Ken Hansen schlug eine neue Task Force unter Komers Leitung, die Ersetzung von Holmes und Yatsevitch durch pro-reformistische US-Offizielle und die Wiederaufnahme der „massiven Intervention“ vor - der Reformeifer war allerdings in der Administration schon lange verflogen. ${ }^{72}$

Die Befürworter einer solchen Politik - bezeichnenderweise in einem von Komers Memoranden als „Douglasites“ tituliert - hatten letztlich nur einen minimalen Erfolg vorzuweisen. Wenigstens gewannen sie die Unterstützung des Attorney General zugunsten zweier gegen das Schah-Regime opponierenden, iranischen Studentenführern in den USA, deren umgehende Auslieferung Teheran gefordert hatte. ${ }^{73}$ Das State Department hatte sich

\footnotetext{
${ }^{71}$ Komer Memo, 22.8.62, FRUS 61-63, XVII-XVIII-XX-XXI Suppl., Dok. 96; Douglas ging so weit zu behaupten: „Persia is really under the control of the CIA.“; ein Beispiel für die erratische Deklassifizierungspolitik ist, dass dieser Passus in einer für die vorliegende Arbeit im Jahr 2004 freigegebenen Version dieses Memorandums geschwärzt wurde, obwohl das Dokument bereits 1996 vollständig im FRUS Supplement publiziert worden war. Im Dezember brachte Douglas den ihm persönlich befreundeten Stammesführer Nāser Xān Ġašgā $\mathrm{i}$ zu einem Gespräch mit Komer. Gašğā' i gab sich als Führer der gesamten liberalen wie konservativen Opposition in Iran aus und kündigte eine baldige Revolution unter seiner Führung an. Vgl. Komer Memo, 15.12.62, ,Iran, 1961-1962 [Folder 1 of 2]', NSF, RWK, Box 424, JFKL.

${ }_{72}$ Vgl. Hansen Memo, 7.11.62, ebd.; Komer schlug als Nachfolger Holmes‘ einen liberalen wie einflussreichen, den Reformideen gegenüber aufgeschlossenen Offiziellen vor, z.B. Bowles oder Schlesinger Jr. Zudem forderte er ein „,crack team“ für die Botschaft, z.B. Bill Polk vom Policy Planning Council, mit dem Ziel, die Umsetzung eines weit reichenden Reformprogramms zu erzwingen: „Let's do a really hard-hitting job of political 'meddling' - fingering as many people as possible who have some potential, engineering them into a position of some prominence (Minister of Interior, Governor of Central Bank, Plan Organization, etc), provide backstopping by staffing with young technocrats, and coach them on how to build strategic political strength (perhaps by reaching beyond the befuddled 'leaders' of the National Front and attempting to capture the broader base of middle level people who would rather be classed as National Front as an expression of protest but who in reality are looking for leaders and a program.)“; Hansen zufolge benötigte die Umsetzung eines solchen Programms einen ,damn good CIA operator."

${ }_{73}$ Vgl. FRUS 61-63, XVIII, S. 189 Anm. o.N.; nachdem sich die amerikanische Einwanderungsbehörde für eine Duldung der beiden Studentenführer ausgesprochen hatte, instruierte das State Department im Mai die Botschaft, Zusagen der iranischen Regierung zu erbeten, auf eine Bestrafung der Studenten nach einer Rückkehr nach Iran zu verzichten. Im August wandte sich der Under Secretary Ball direkt an Robert Kennedy mit dem Hinweis ,"the continued presence of the two Iranian students in the United States is, in the view of the Department of State, detrimental to our relations with Iran."; Vgl. State \#843, 15.5.62, ,Iran [...] 5/2/62“; Tehran \#951, 22.5.62, ,Iran [...] 6/30/62‘, NSF, Countries, Box 116, JFKL; Miner Memos, 4./14.5.62; , Office Memoranda, 1962‘, NEA, GTI: RIAD, 1958-63, Box 4; Christenson Memo, 13.8.62; Draft Letter Ball, 28.8.62, ,13-A. [...] Qotbzadeh, 1962‘, ebd., Box 7; Komer erinnerte sich später, dass ,a couple of real feudal barons from Iran, the Kashgai brothers,
} 
zuvor für eine Ausweisung der beiden Studentenführer ausgesprochen, neben anderen Argumenten auch darauf hinweisend, dass eine, wie auch immer unwahrscheinliche, zukünftige Regierung der Nationalen Front in Iran aufgrund ihrer Führungsschwäche und Zerrissenheit nicht lange in der Lage sein würde , \{to; R.P. $\}$ long postpone a movement toward neutralism, anti-Westernism, and an expansion of communist influence, as well as hostility to Western oil interests. “74 Robert Kennedy ließ sich von solchen Argumenten allerdings nicht überzeugen. Nachdem eine von ihm angeregte FBI-Untersuchung die gegen beide Studenten vorgebrachten pro-kommunistischen Verbindungen widerlegen konnte, informierte er sogleich Dean Rusk ,to go chase himself.“75 Tatsächlich gab das State Department auch in der Folgezeit nicht seine Bemühungen um die Ausweisung der oppositionellen Iraner auf - symptomatisch für die langfristigen Folgen für die US-Politik war dabei, dass es sich bei einem der beiden Studentenführer um Sādeg Ġotbzādeh handelte, den späteren Außenminister (1979-80) des revolutionären Iran. ${ }^{76}$

Selbst wenn die Administration wenigstens in dieser Detailfrage den eigenen liberalen Idealen (vorerst) treu blieb, so war dennoch nicht zu übersehen, dass der Haupttrend der amerikanischen Iranpolitik in eine gänzlich andere Richtung wies. Ein unmissverständliches Signal an die verbliebenen Reformisten war schließlich der Ausgang der Debatten über das MAP-Programm.

began giving Justice Douglas (who is very naive) a lot of bull about how they were great liberals and the Shah of Iran was a big reactionary."; Robert W. Komer OHI, 22.12.69, JFKL, S. 42f.; vgl. auch MATIN-ASGARI, Iranian Student Opposition, S. 48f.; Richter Douglas hatte in einem Brief an den Präsidenten vom Februar gewarnt, die iranische Regierung wolle die Deportation kritischer Studenten durchsetzen, um diese dann vermutlich hinrichten zu lassen. Zudem plane der Schah, Amini durch Baxtijār zu ersetzen, was in eine Terrorherrschaft münden würde. Vgl. Douglas Letter, 22.2.62, encl. to Dungan Memo, 2.3.62, ,Iran, Security 1961-1963‘, POF, Countries, Box 119 , JFKL.

${ }^{74}$ State Report, 20.8.62, encl. to Grant Memo, 20.8.62, ,13-A. Students [...] 1962‘, NEA, GTI: RIAD, 1958-63, Box 7; offenbar hatte man seitens des State Department auch eine Untersuchung der Aufenthaltsberechtigung 'Ali Šājegāns, eines anderen prominenten Führers der Nationalen Front in den USA durch das Justizministeriums angeregt, um dessen Ausweisung als „Subversiver“ zu erreichen. Vgl. Justice Department, Immigration and Naturalization Service, Report of Investigation: Ali Shayegan, 15.3.62; Tehran Operations Memo \#OMV-8, 12.7.62, DNSA: IR00445; Bowling hatte sich bereits im Januar vehement für die Abschiebung der Studenten eingesetzt. Zudem sei Iran eines der „,freiesten“ Länder in der nicht-westlichen Welt. Kritik an der Regierung werde toleriert und es gebe so gut wie keine staatliche Willkür oder Brutalität der Polizei. Vgl. Bowling Memo, 23.1.62, 788.00/1-2362, CF 60-63.

${ }^{75}$ Zit. aus William O. Douglas OHI, 13.11.69, JFKL; vgl. auch ders., Court Years, S. 308; BILL, Eagle and Lion, S. 133; zu ähnlichen Vorgängen in der Bundesrepublik Deutschland vgl. Abteilung 6/Referat 604 an Herrn D5, ,Politische Demonstrationen ausländischer Studenten in der Bundesrepublik', 20.2.61, B 12/992, PAAA.

${ }_{76}$ Zur Deportationsproblematik vgl. ausführlich SHANNON, Losing Hearts and Minds, S. 100-10; offensichtlich hatte Robert Kennedy das Department of Justice angewiesen, dem State Department in Zukunft Auskünfte über den Status der Studenten zu verweigern und ,to treat them in the same way as Cuban refugees are treated“; das State Department ging so weit zu behaupten, die weitere Duldung der oppositionellen Studenten könnte dem Schah anzeigen, ,that there are powerful elements in the U.S. Government who are seeking his overthrow"; Talbot Memo, 8.10.62, ,13-A. [...] 1962‘, NEA, GTI: RIAD, 1958-63, Box 7 RG 59, NACP; zu Gotbzādeh vgl. auch BILL, Eagle and Lion, S. 266; das CBS-Nachrichtenmagazin 60 Minutes sendete 1977 einen Bericht, der Pläne der SAWAK zur Ermordung Gotbzādehs nahelegten, damals auch vom ehemaligen CIA-Offiziellen Richard Cottam bestätigt. Vgl. Department of State, Public Affairs Staff, ,60 Minutes Transcript‘, 6.3.77, DNSA: IR01153; zur späteren angeblichen Beteiligung G̈otbzādehs an einer Verschwörung gegen Khomeini und seiner Hinrichtung vgl. Abrahamian, Tortured Confessions, S. 155-9; sowjetische Quellen behaupten eine sowjetische Desinformationskampagne in diesem Zusammenhang. Vgl. ANDREW/MITROKHIN, Mitrokhin Archive II, S. 186; KUZICHKIN, Inside the KGB, S. 303f; im Gegensatz zu den der Nationalen Front nahestehenden Studentengruppen zeigte man sich gegenüber den Mozaffar Bagāē $i$ zugehörigen Organisationen sehr kulant, die immer wieder als mögliche ausführende Organe für eigene Interessen Erwähnung fanden. Siehe z.B. das Gespräch mit dem Studentenführer Mansur Rafi zādeh in Crawford Memo, 5.6.62, ,13-A. Student [...] 1962‘, NEA, GTI: RIAD, 1958-63, Box 7; Rafi' zādeh war später der SAWAK-Leiter in den USA und stand offenbar auch im Sold der CIA. Vgl. GASIOROWSKI, Client State, S. 119 n65; vgl. RAFIZADEH, Witness, S. 99-112. 
Das Planning Team der JCS unter Leitung General Twitchell hatte im Anschluss an die Beratungen vor Ort in Teheran eine Empfehlungsliste für das zukünftige Programm zusammengestellt. Den iranischen Bedenken hinsichtlich einer zu weit gehenden Reduktion der Sollstärke war man etwas entgegen gekommen: Statt 150.000 Mann gab man sich auch mit einem Rückgang auf 162.000 Mann innerhalb von 2-3 Jahren zufrieden. Darüber hinaus versprach man nun auch die Lieferung eines vom Schah gewünschten modernen Radarsystems in Kooperation mit Großbritannien. Allerdings wurde auf die extravaganten Wünsche des Schahs nach Phantom-Jägern und Ähnlichem nicht eingegangen. Tatsächlich gelang es durch einige Einsparungen, das Gesamtprogramm innerhalb der vereinbarten Obergrenze von $\$ 300$ Millionen zu halten. Holmes wurde zugleich instruiert, die Iraner zu warnen: Sollte das Modernisierungsprogramm unverhältnismäßige Steigerungen im Militäretat Irans auslösen, würde man von US-Seite mit einer Verlangsamung des Programms reagieren. Zudem sollte Holmes erneut die Priorität innerer Reformen betonen. ${ }^{77}$ Wie zu erwarten, weigerte sich Holmes, die ihm nicht genehmen Instruktionen umzusetzen. Stattdessen plädierte er noch vor der Präsentation des MAP-Programms beim Schah für eine Revision. Der Schah erwarte, so der Botschafter, aufgrund der während seines Washington-Besuchs aufgebauten besonderen persönlichen Beziehung zu Präsident Kennedy Zugeständnisse. Ein völliges Ignorieren seines Briefes vom Juli würde sicherlich als Affront verstanden. Daher forderte Holmes eine „Restitution“ der beiden zuvor gestrichenen Fregatten als positive Geste. Die MAPVerhandlungen würden die zukünftigen iranisch-amerikanischen Beziehungen entscheidend vorherbestimmen. Ein Entgegenkommen sei daher unbedingt geboten, da die Fregattenfrage „\{concerns; R.P. $\}$ our relations with the Shah, which are in large measure our relations with this country“, so die entlarvende Feststellung des Botschafters. ${ }^{78}$ Komer wandte sich intern energisch gegen Holmes` Forderung. Ein Nachgeben in dieser Grundsatzfrage würde dem USPrestige schaden - zudem wäre eine Zusage der Fregatten ein weiteres Signal an den Schah, die amerikanischen Beteuerungen über die Priorität innerer Reformen nicht weiter ernst zu nehmen. Vergebens forderte er von Kennedy ein „Hell no“. Das Weiße Haus gab dennoch nach, nicht zuletzt da Außenminister Rusk bereits im Vorfeld McNamara von der (politischen) Notwendigkeit der Revision überzeugt hatte. ${ }^{79}$ Holmes ignorierte zugleich parallele Instruktionen, den Schah unmissverständlich von weiteren Forderungen abzubringen und,

\footnotetext{
77 Vgl. Fuqua Jr. Memo, 26.4.62, ,Iran 1962 000.1---092‘, OASD/ISA Files: General and Country, 1962, Box 89, RG 330; vgl. Gilpatric Letter, 31.8.62; enclosed ,Instructions to U.S. Ambassador to Iran', 788.5/8-3162; Joint State-Defense \#277, 8.9.62, 788.5/9-862, CF 60-63; tatsächlich sah die Weisung auch eine Umorganisation der iranischen Streitkräfte in mobile Einheiten mit höherer Feuerkraft vor ,to provide a tailored force for the defense of the northern mountainous border areas."; vgl. Talbot Letter, 13.9.62, FRUS 61-63, XVIII, S. 90f.; ebd., S. 91 $n 1$; vgl. W. Bundy Memo, 16.10.62, ,Iran 1962 000.1---092‘, OASD/ISA Files: General and Country, 1962, Box 89, RG 330, NACP.

${ }_{78}$ Tehran \#404, 14.9.62, 788.5/9-1462, CF 60-63; im Vorfeld der eigentlichen MAP-Verhandlungen mit den Iranern gebärdete sich Holmes zunehmend als Anwalt des Schahs. Vgl. Joint State-Defense \#243, 31.8.62, 788.5/8-2762; Tehran \#317, 1.9.62, 788.5/9-162, CF 60-63.

${ }^{79}$ Vgl. Komer Memo, 15.9.62; Draft Bundy Memo, 17.9.62, ,Iran, 1961-[...] Memoranda', NSF, RWK, Box 424, JFKL; State \#310, 18.9.62, FRUS 61-63, XVIII, S. 99; für die Hintergründe der Entscheidung siehe ebd., Anm. o.N.; tatsächlich war die Streichung der beiden Fregatten der vielleicht umstrittenste Punkt im MAP-Programm. Vgl. Jack C. Miklos OHI, 28.7.86, OHIC, S. I-68; W. Bundy Memo, 16.10.62, ,Iran 1962 000.1 ---092‘, OASD/ISA Files: General and Country, 1962, Box 89, RG 330, NACP.
} 
wieder einmal, auf das Primat innerer Reformen mit Nachdruck zu verweisen. Zwar akzeptierte der Schah das revidierte MAP-Programm. Komer sagte dennoch konsterniert voraus, man könne ,[...] expect more pleas from Pahlavi all too soon. “80

Im Anschluss an diese symbolische Niederlage unternahm Komer in seiner Rolle als wichtigster Repräsentant der reformistischen Interventionisten im Oktober 1962 einen letzten Versuch, den immer sichtbarer werdenden Trend in der US-Iranpolitik umzukehren. In einem die bisherige Politik zusammenfassenden langen Memorandum an Präsident Kennedy warnte Komer mit Nachdruck vor allzu großer Gelassenheit angesichts der Lage in Iran. Die scheinbare Ruhe sei, so zeige die Erfahrung, nur eine Ankündigung des nächsten Sturms. Chruschtschows Vorhersage eines internen Zusammenbruchs könne durchaus noch eintreffen - Iran sei weiterhin ,one of the most vulnerable soft spots round the Bloc periphery.“ Doch die USPolitik sei zuvorderst reaktiv und abwartend:

For fifteen years we've pursued an essentially reactive policy. We've rushed into the breach whenever a clear threat reminded us of Iran's crucial position (as in the 1946-47 Azerbaijan crisis, the Mossadegh period, the Shah's 1958 flirtation with the Soviets, or the May 1961 crisis). But as each was over, we relapsed into an essentially passive acceptance of things Iranian as they are. ${ }^{81}$

Kaum sei in Iran nach der akuten Krise vom Frühjahr 1961 Ruhe eingekehrt, habe man sich sogleich zurückgezogen und die systematische Demontage Aminis durch den Schah widerstandslos registriert - stattdessen habe man sich vorwiegend mit dem MAP-Programm beschäftigt. Das eigentliche Ziel, eine ,controlled revolution“ von oben durchzusetzen, um eine gewalttätige ,uncontrolled one“ zu vermeiden, sei weiterhin gefährdet. Die radikalen Vorschläge à la Douglas lehnte Komer aber ab. Eine durch die USA erzwungene Abdankung bzw. Unterstützung für den gewaltsamen Sturz des reformunwilligen Mohammad Rezā sei keine Option. Angesichts der Schwäche und Uneinigkeit der Opposition - gemeint war die Nationale Front - sei in diesem Fall mit schwachen und instabilen Regierungen bzw. unter Umständen mit der Errichtung einer diktatorischen Militärregierung zu rechnen. Eine schwächliche neutralistische Regierung aber würde zwangsläufig zu einem Einflussgewinn der Sowjetunion führen. ${ }^{82}$ Zudem sei anzunehmen, dass ,[...] in the absence of even the modest

\footnotetext{
${ }^{80}$ Komer Memo, 24.9.62, ,Iran General 9/24/62-9/30/62‘, NSF, Countries, Box 116, JFKL; der Schah äußerte neue Wünsche z.B. nach höherer Sollstärke, drei weiteren Panzer-Bataillonen und zusätzlichen Radarstationen und er versuchte erneut, Alternativen zur F-5 zu erreichen. Zudem verlangte er ein schriftliches Zugeständnis, dass bei einer Veränderung der Sicherheitslage das Programm noch vor Ablauf der Fünfjahresfrist revidiert werden könne. Offenkundig erwähnte Holmes innere Reformen mit keinem Wort. Vgl. MemCon, 19.9.62, FRUS 61-63, XVIII, S. 100-5; Holmes Memo, 19.9.62, ebd., S. 105-9; ebd., S. 105 Anm. o.N.; Tehran \#430, 22.9.62, 788.5/92262, CF 60-63; vgl. JCS History Iran, S. 71f.

${ }^{81}$ Komer Paper, ,Our Policy in Iran', 20.10.62, FRUS 61-63, XVIII, S. 189-95, hier 190; Komer verteilte seine Grundsatzkritik an McGeorge Bundy und einige andere Offizielle. Er reichte das Papier zudem an Bobby Kennedy weiter ,to counter flak he's getting from Douglasites“; ebd., S. 189, Anm. o.N.

${ }^{82}$ Komer sah die USA durchaus in der Lage ,, $\{$ to; R.P. $\}$ bring down the Shah, simply by withdrawing aid and letting nature take its course. If this weren't enough, US could press for elections and a new Majlis, disengage from its present close relations with the court, invite National Front leaders to US, etc. The Shah in desperation might flirt with the Soviets or try open dictatorship, but would more likely flee.“; ebd., S. 191.
} 
source of stability the monarchy and army now provide, we'd have less leverage with whatever regime eventually emerged." ${ }^{83}$ Somit blieb aus Sicht Komers als einzige gehbare Alternative für die amerikanische Politik die Fortsetzung von Aminis Reformprogramm mit Unterstützung des Schahs, aber angeregt und wesentlich beschleunigt durch Hilfestellung und Druck seitens der Führungsmacht. Anders als in Griechenland, Pakistan oder der Türkei gebe es in Iran keine „strongminded and reasonably competent leadership.“:

Therefore, an essential corollary to supporting the present regime is that the US must pursue a policy deliberately calculated to maximize its chances of its survivability and popular acceptability. If we're going to support it we must simultaneously make every effort to enhance its effectiveness by actively pushing, prodding, and cajoling it in directions we favor, rather than bailing it out only when its own mismanagement gets it in a box. We must attempt to reshape the regime to give it greater life expectancy than the 3-5 years it otherwise has. ${ }^{84}$

Letztlich forderte Komer eine Rückkehr zur Task Force-Politik, ein aktivistisches und interventionistisches Programm gerichtet auf eine möglichst ganzheitliche Umsetzung der diversen Reformideen mittels seitens der USA erzwungener Reformkabinette, eine „Serie von Aminis“, oder am besten sogar durch eine Rückkehr Aminis selbst. ${ }^{85}$ Man dürfe keine Angst vor Intervention und „Einmischung“ haben. Tatsächlich sei man so eng mit dem bestehenden Regime verbunden, dass es von den meisten Iranern ohnehin mit dem ,unsichtbaren“ Einfluss der USA gleichgesetzt werde. ${ }^{86}$

Komers Hoffnungen, mit diesem Memorandum an Kennedy eine neue Iran exercise ins Laufen zu bringen, blieben unerfüllt. Der Präsident griff Komers Vorschläge vom Oktober nicht auf und verzichtete in einem persönlichen Treffen mit Botschafter Holmes offenbar darauf, weiteren Druck auf den Schah hinsichtlich Reformen anzukündigen. ${ }^{87}$ Symptomatisch für die fehlende Bereitschaft der Administration, ein neues Reformexperiment in Iran zu wagen, war die nahezu kritiklose Akzeptanz eines NEA-Entwurfs für Guidelines for Policy and Operations

\footnotetext{
${ }^{83}$ Ebd., S. 192.

${ }^{84}$ Ebd., S. $192 \mathrm{f}$.

${ }^{85}$ Aminis Amtszeit sei, so Komer, tatsächlich alles andere als ein „Flop“ gewesen. In 15 Monaten habe er mehr erreicht als alle seine Vorgänger in 15 Jahren. Tatsächlich unterschätzten die USA ihre Einflussmöglichkeiten gegenüber dem Schah: ,[...] he needs us and knows it (and he's never had a strong lead.)“ (S. 193).

${ }_{86}$ Ebd.; für die Umsetzung dieser Politik müssten allerdings personelle Wechsel in Betracht gezogen werden. Holmes z.B. sei zu sehr ,Traditionalist“ für diese Art der Politik. Komer sprach sich gegen eine neue Task Force oder ein weiteres NSAM aus und präferierte eine direkte Weisung Kennedys an Rusk und ein direktes Mandat für Komer selbst. Komer informierte den Attorney General über die dem Memorandum zugrunde liegende Idee: ,We all agree that the US must do more than it's doing, but are leery of such radical solutions as pressing for a Majlis or backing the National Front, with the Shah relegated to figurehead status or replaced by a regency. We stick with realpolitik." \{Hervorheb. i. Orig.\}; Komer Memo, 20.10.62, ,Iran, 1961-1962, White House Memoranda“, NSF, RWK, Box 424, JFKL.

87 In einem weiteren Memorandum an den Präsidenten hatte Komer die Einschätzungen Holmes` über Iran grundsätzlich in Frage gestellt und ihn ein weiteres Mal auf das Ignorieren präsidentieller Instruktionen durch den Botschafter hingewiesen. Vgl. Komer Memo, 13.11.62, ,Iran General 11/62‘, NSF, Countries, Box 116A; Komer war im Anschluss enttäuscht über das Ausbleiben einer Reaktion Kennedys und dessen Nichterwähnen des Gesprächsinhalts mit Holmes. Eine Neuauflage des Amini-Experiments sollte ohne den störrischen Botschafter durchgeführt werden. Komer dachte an George McGhee als einen möglichen Kandidaten für die Nachfolge von Holmes. Er schrieb resignierend an Bundy, , there's not much I can do till you and JFK decide whether my recipe worth trying."; Komer Memo, 21.11.62, ,Iran, 1961-1962, White House Memoranda', NSF, RWK, Box 424; Komer Memo, 27.11.62, ,Staff Memoranda, Robert Komer 11/62-12/62‘, NSF, M\&M, Box 322, JFKL.
} 
für Iran vom September 1962, eine Ergänzung des Task Force Report sowie der aktuellen Antwort auf NSAM N ${ }^{\circ} 67$ vom Januar $1962 .{ }^{88}$ Die Guidelines revidierten nun auch formal die meisten Grundsätze der vorherigen Task Force-Politik. Nicht mehr ,vigorous action“ seitens der USA und das Erzwingen rascher und umfassender Reformen wurde angestrebt. Stattdessen empfahl man zwar eine Fortsetzung der Reformpolitik, vorzugsweise wieder unter einem reformistisch gesinnten Regierungschef, nun allerdings in vorsichtigem Tempo unter konservativer Steuerung und unter Verzicht auf sozialrevolutionäre Zielsetzungen. Nirgends war mehr von „kontrollierter Revolution“ die Rede. ${ }^{89}$ Rudimentär waren auch noch die Ideen einer neuen politischen Synthese für Iran vorhanden. Gemäßigte Nationalisten und Teile der konservativen Opposition sollten mittelfristig von der Notwendigkeit der Kooperation mit dem bestehenden Regime überzeugt werden - amerikanische Einflussmittel sollten weiterhin zugunsten der Transformation gerade der ,urban middle class“ in eine ,constructive social and political force“ eingesetzt werden. ${ }^{90}$ Die gegenwärtige nationalistische Opposition, deren Integration in die Regierungsgeschäfte noch ein Kerngedanke des reformistischen US-Ansatzes gewesen war, war allerdings nach Auffassung der Autoren der Guidelines politisch vollkommen inakzeptabel, in erster Linie geprägt von anti-westlicher Demagogie nach dem Muster Mosaddeġs. Eine Regierung dominiert durch die Nationalisten ,[...] poses unacceptable risks of chaos and the growth of Communist influence to the point of domination."91 Die Politikempfehlungen der Guidelines waren zudem gleichbedeutend mit der Aufgabe des Grundgedankens hinter dem Task Force Report. Statt die fast ausschließliche Konzentration auf den Schah zu reduzieren, empfahl das Papier vielmehr an erster Stelle zu ,maintain the Shah in a position of power" und seine pro-westliche und anti-kommunistische Orientierung zu bestärken. Angesichts dieser weitreichenden Identifikation der US-Politik mit dem iranischen Monarchen mussten die anderen Empfehlungen zweitrangig erscheinen, wie z.B. den Schah zur Fortsetzung des Reformprogramms und zur Einnahme einer weniger exponierten Stellung zu

\footnotetext{
${ }^{88}$ Die Guidelines waren eine Antwort auf die Auflösung des OCB und die Einstellung der regionalen bzw. Länderbezogenen NSC-Direktiven aus der Eisenhower-Zeit. Vgl. Bowles Memo, 31.10.61, FRUS 61-63, XXV, S. 36f.; vgl. auch FRUS 61-63, XVII, S. $360 n 2$; vgl. auch Talbot Memo, 30.3.62, ,I.A Guidelines'; Talbot Memo, 8.10.62, ,Memos Thru S/S for Clearance, 1962‘, NEA, GTI: RIAD, 1958-63, Box 4; und Battle Memo to All Assistant Secretaries, ,Guideline Papers‘, 9.11.61, ,Policy Statement Guidelines', Lot 67 D 496, G/PM, Combined Policy Office, Subject Files 1961-66, Box 2; die hier verwendete Version der Guidelines ist eine gebundene Version vom September 1962 aus den Akten des Policy Planning Council. Sie ist identisch mit einem final draft, in den GTI files. Siehe folgende Anm.; in den Akten findet sich keine Information über eine formale Annahme der Guidelines seitens des NSC oder des Präsidenten als Grundlage für die US-Politik.

${ }^{89}$ Department of State, ,Guidelines for Policy and Operations: Iran', September 1962, ,Iran', Lot 69 D 121, S/P Records: 1962, Box 216; der Bericht empfahl einen „moderate and reformist Prime Minister as a catalytic agent“, allerdings unter Führung des in seiner Machtstellung nun nicht mehr in Frage gestellten Schahs: ,The Shah must support a program of social and economic reform to be carried out by the Cabinet, while he continues to hold the reins of foreign and military policy, and protects the defenseless Prime Minister against the assaults of the 'Nationalists' and the conservatives." $\{$ Hervorheb. v. Verf.\}; ebd., S. 8.

${ }^{90}$ Vgl. ebd., S. 20f.

${ }^{91}$ Ebd., S.8; die Guidelines schlossen allerdings im Anschluss an eine geglückte Transformation der urbanen Mittelklassen und den langfristigen Aufbau einer moderaten politischen Basis unter Beteiligung der bislang politisch apathischen Bevölkerungsmehrheit eine schrittweise Zurückstufung der Rollen des Schahs und der Militärführung auf eine „,more nearly constitutional role“ nicht aus. In einem Memorandum vom Januar hatte Bowling noch seine Sichtweise auf die Akteure drastischer ausgedrückt: ,With his hold over the peasantry and the security forces, the Shah is the sine qua non for genuine reform in Iran. [...] Without Shah, moderation and sensible reform will disappear from the Persian scene within minutes. Results would be bad for Iranian people and bad for U.S.“; Bowling Memo, 23.1.62, 788.00/1-2362, CF 60-63.
} 
bewegen. ${ }^{92}$

Die Guidelines - keine formale Grundsatzentscheidung, aber dennoch aufgrund des ausbleibenden Widerstandes des Weißen Hauses de facto die Richtlinien zukünftiger USPolitik festsetzend ${ }^{93}$ - waren in der letzten Konsequenz eine Rückkehr zur Politik der späten Eisenhower-Administration. Angesichts des Verhaltens Mohammad Rezās während der Ministerpräsidentenschaft Aminis und seiner fortwährenden und nahezu ausschließlichen Konzentration auf militärische Belange waren ernsthafte Zweifel über die Zukunft der Reformanstrengungen, gerade bei Ausbleiben des bislang entscheidenden Drucks seitens der USA, angebracht. Kenneth Hansen warnte im Oktober 1962, dass die ,technokratische Ressource“, gemeint waren die jungen, pro-westlichen und vorwiegend in den USA ausgebildeten Ökonomen und Ingenieure in der iranischen Planorganisation, von Zerfall bedroht sei - damit ginge nicht zuletzt ein erheblicher Einflussverlust der USA einher, da gerade die politischen und wirtschaftlichen Zielsetzungen dieser Gruppe den amerikanischen Vorstellungen in vielem entsprachen. Allerdings war mit dem nun absehbaren Zurückfahren der Wirtschaftshilfe der US-Einfluss auf die iranische Entwicklungspolitik zunehmend eingeschränkt - angesichts der gescheiterten reformistischen Intervention war ohnehin der Wille zur fortgesetzten Unterstützung des pro-amerikanischen Netzwerkes in Iran eher limitiert. Bowling in GTI widersprach einer Fortsetzung des Amini-Experiments vehement. Die Technokraten wären unter Amini praktisch die „Finanzdiktatoren“ Irans gewesen und hätten nunmehr ihr gesamtes Prestige aufgebraucht: „The one almost universal reaction among Iranians of all classes toward the end of the Amini period was deep contempt and dislike for 'the bow-tie set', whom they regarded as American agents. “94

\footnotetext{
${ }^{92}$ Vgl. Guidelines, S. 18.; der Schah wurde als ,brilliant but sometimes emotional and erratic combination of European intellectual and Oriental intriguer“ beschrieben. Ebd., S. 3; tatsächlich war der Auftrag zum Machterhalt Mohammad Rezās bereits in einem früheren, nahezu identischen Entwurf der Guidelines vom März 1962 enthalten. Vgl. den von Bowling verfassten Entwurf vom 27.3.62 in ,I.A Guidelines', NEA, GTI: RIAD, 1958-63, Box 5, S. 11 .

${ }_{93} \mathrm{Im}$ Office of Politico-Military Affairs interpretierte man die Bedeutung der Länder- und Regionen-Guidelines als eher fragwürdig und von ungewisser Bedeutung, hielt sie aber auf keinen Fall für einen gleichwertigen Ersatz für die früheren NSC-Studien der Vorgängeradministration. Die Frage der Bedeutung der (insgesamt offenbar 113) Guidelines Papers wurde offenbar nie geklärt - dennoch und trotz der relativ niedrig angesiedelten Beschlussebene der Assistant Secretaries gaben die Guidelines die generellen Richtlinien als ,,concise formulation of basic US policy toward each area and country“ vor. Zit. aus Baxter Memo, 24.10.61, ,Policy Statement Guidelines', Lot 67 D 496, G/PM, Combined Policy Office, Subject Files 1961-66, Box 2; vgl. auch Rostow Memo, 5.8.61; Magathan Memo, 21.12.61; Weiss Memo, 22.12.61; ebd.; bemerkenswert ist, dass man zumindest im Defense Department die Guidelines als autoritative Richtlinien interpretierte. Vgl. JCS History Iran, S. 72; eine Kopie der Guidelines vom September 1962 findet sich auch in den Akten des Office of the Assistant Secretary of Defense for International Security Affairs (ISA). Siehe die Kopie angehängt an Brubeck to W. Bundy, 4.12.62, ,Iran 1962 000.1---092‘, OASD/ISA Files: General and Country, 1962, Box 89, RG 330, NACP; ein weiteres Problem war das ungeklärte Verhältnis zwischen den Guidelines des State Department und den Aktivitäten anderer Behörden wie z.B. den von Kennedy angeforderten Studien der JCS im Rahmen der „Cold War Activities“; vgl. Bundy Letter, 3.7.63; Kitchen Memo, 11.4.63, ,State-JCS Meeting, April 12, 1963", Lot 70 D 328, State-JCS Meetings: 1959-63, Box 3 .

${ }_{94}$ Bowling Memo, 31.10.62, ,Office Memoranda, 1962‘, NEA, GTI: RIAD, 1958-63, Box 4; Papanek Memo to Mason/Vernon, 11.10.62, encl. to Hansen Memo, 26.10.62, ,Iran, 1961-1962 (Folder 1 of 2)‘, NSF, RWK, Box 424, JFKL; Bowling lehnte dagegen jedwede direkte US-Unterstützung für eine ihm zufolge unpopuläre „Schattenregierung“" grundsätzlich ab. Eine ähnliche Einschätzung bezüglich der pro-amerikanischen Technokraten und Aminis gab die britische Botschaft vor Ort. Es gebe nun in Iran ,, [...] widespread resentment at American interference in all sectors of Iranian life.“; Harrison to FO, No. 9, 21.2.62, PREM 11/3846, TNA:PRO.
} 
Letzten Endes setzte sich die Sichtweise Bowlings durch: Eine Bereitschaft der KennedyAdministration, aktivistisch auf eine Umsetzung weitreichender Reformen zu drängen, war kaum noch vorhanden. Erstaunlich angesichts der Vorgeschichte US-amerikanischer Einwirkung auf innere Entwicklungen Irans ist die fast widerstandslose Preisgabe wichtiger Bestandteile des pro-amerikanischen Netzwerkes, offenbar der Einsicht geschuldet, dass die Möglichkeit externer Mächte, entscheidend Planungs- und Umsetzungsprozesse in anderen Staaten zu beeinflussen, letztlich allzu begrenzt war. ${ }^{95}$ Der Defätismus - eine zutreffende Bezeichnung zumindest was den verbliebenen Willen zur reformistischen Intervention angeht - im Herbst 1962 war in vielerlei Hinsicht nur die konsequente Fortsetzung des bereits oben beschriebnen Erlahmens dieses Willens zur Jahreswende 1961/1962. Die „Politics of Massive Intervention" in peripheren Gesellschaften der sogenannten Dritten Welt - die Durchsetzung von „Revolutionen von Oben“ mittels des Einsatzes sämtlicher offener wie verdeckter zur Verfügung stehender Mittel mit dem hauptsächlichen Ziel von „rapid and sustained development" - war im Kern, wenn auch nie formal, bereits Anfang 1962 aufgegeben worden. ${ }^{96}$ Zwar hatte die Amini-Regierung einige wichtige Schritte eingeleitet und auch sein Nachfolger 'Alam setzte die Reformen fort, wenn auch in Abhängigkeit und gelenkt vom Hof. Von einer erfolgreichen Umsetzung der Reformziele konnte jedoch zu diesem Zeitpunkt keine Rede sein. Im Gegenteil, die CIA hatte bereits nach dem Rücktritt Aminis gewarnt, der Schah werde nun versuchen , $[\ldots]$ to press ahead by himself, sincerely trying to implement the anticorruption and land reform programs begun by Amini, but without either the will or the ability to deny special treatment to those whom he trusts or to whom he is bound by personal ties." Ebenso zweifelhaft wie die Zukunft der Reformen war eine mögliche Rückkehr zu einer eher verfassungskonformen Regierungsform. Die CIA rechnete nicht mit der baldigen Abhaltung von Wahlen. In einem Gespräch mit dem CIA Station Chief Yatsevitch deutete der Schah zudem Besorgnis erregende Ambitionen an - im Falle eines Scheiterns auch der 'AlamRegierung müsse man ,[...] a drastically new approach to the method of governing Iran“ in Erwägung ziehen. ${ }^{97}$

Trotz der weiterhin bestehenden Abhängigkeit von den USA bezüglich Wirtschafts- und Militärhilfe war der Schah zudem in Reaktion auf das Verhalten Washingtons während des

\footnotetext{
${ }^{95}$ CIA Station Chief Yatsevitch wurde persönlich vom Schah informiert, angesichts der weit verbreiteten antiamerikanischen Stimmung im Land müssten einige vorwiegend symbolische Maßnahmen gegen den US-Einfluss unternommen werden. Bezeichnenderweise verstand Yatsevitch die Bemerkung als Seitenhieb auf die jungen Technokraten, die in der iranischen Presse fortwährend als „American chickens“ verspottet wurden. Der CIAMann wies daher auf, ,really important contributions“ unter anderem der Planorganisation hin und erhielt daraufhin von Mohammad Rezā die Zusage, dass es zu keiner Verfolgung dieses Personenkreises kommen werde. Vgl. Yatsevitch Memo, 13.8.62, ,I. The Shah \& Court [...]', Box 4, NEA, GTI: RIAD, 1958-63, Box 5.

${ }^{96}$ Siehe oben, S. 225f.; ,rapid and sustained“" in Bureau of the Budget, Staff Report: "Survey of the Alliance for Progress Program in Brazil, Argentina, Chile and Bolivia', 7.8.62, encl. to Belk Memo, 14.8.62, ,Bureau of the Budget General, Summary of a Survey...', NSF, D\&A, Box 270, JFKL, S. 3.

${ }_{97}$ CIA, SNIE 34-62, 7.9.62, FRUS 61-63, XVIII, S. 86; Yatsevitch Memo, 13.8.62, siehe Anm. 95; in einem weiteren Gespräch mit Yatsevitch hatte der Schah den früheren Ministerpräsidenten Šarif-Emāmi mehrfach lobend erwähnt. Yatsevitch und Holmes vermuteten daher eine baldige Ablösung 'Alams. Vgl. Yatsevitch Memo, 18.9.62, ,Iran, 1961-1962 [Folder 1 of 2]', NSF, RWK, Box 424; Tehran \#451, ,Iran General 9/24/62-9/30/62‘, NSF, Countries, Box 116, JFKL.
} 
„Amini-Experiments“ nunmehr entschlossen, sich von der Vormacht wenigstens symbolisch zu emanzipieren. Wirkungsmächtig war ohne Frage die bereits beschriebene Beschränkung der Handlungsfreiheit des pro-amerikanischen Netzwerks. In einer symbolischen Distanzierung vom Patron setzte der Mohammad Rezā zudem ein begrenztes Rapprochement gegenüber der Sowjetunion durch. Die Anfang des Jahres aufgenommenen Gespräche mit Moskau kamen am 15. September 1962 zum Abschluss: Iran verpflichtete sich in einer unilateralen Deklaration, die Stationierung von Raketen fremder Staaten auf dem eigenen Territorium nicht zuzulassen. In der späteren Forschung bisweilen als „crucial event“ gewertet, war die zeitgenössische Reaktion in Washington allerdings eher gelassen. ${ }^{98}$ Militärstrategisch war die Entwicklung unbedeutend - überrascht war man eher über die unerwartete, wohl im Zusammenhang mit Kuba stehende sowjetische Kompromissbereitschaft. Leichte Beunruhigung entstand durch die offenkundige Popularität des Schrittes bei der iranischen Öffentlichkeit. Eine mögliche neutralistische Wende des Schahs aber befürchtete niemand - zu offenkundig und im Grunde unumkehrbar war die Westorientierung Irans. ${ }^{99}$

Das Verhalten des Schahs während der Budgetkrise im Sommer, seine fortgesetzte, fast ausschließliche Aufmerksamkeit für militärische Belange und seine Ablehnung weiterer Experimente mit „starken“ Ministerpräsidenten verhießen für die Zukunft wenig Gutes. ${ }^{100}$ Angesichts der nahezu unveränderten Gesamtlage in Iran und der Rückkehr, de facto, zur persönlichen Herrschaft Mohammad Rezās war die (endgültige) Aufgabe der Task Force-

\footnotetext{
${ }_{98}$ Siehe oben, S. 291; „,crucial event“ in BILL, Eagle and Lion, S. 150; die Zusage lautete konkret, Iran ,„[...] will give to no foreign power the right of having missile bases of any type on the territory of Iran."; die Sowjets hatten nicht nur ihre vorherige Forderung einer bilateralen Vereinbarung fallengelassen, sondern verzichteten auch auf eine mit der Deklaration einhergehende Klarstellung. Teil der Klarstellung wäre eine Zusage Teherans gewesen, wonach sich Iran nie als Mittel einer Aggression gegen die UdSSR instrumentalisieren lasse, wohl angelegt an die entsprechenden Klauseln des Vertrages von 1921. Außenminister Ārām gab stattdessen eine öffentliche Erklärung in diesem Sinne ab. Eine weitere Klarstellung hinsichtlich des zukünftigen Erwerbs von Raketen durch Iran wurde durch Intervention von Holmes gemeinsam mit dem britischen Botschafter verhindert, da sie offenkundig gegen eine mögliche nukleare Teilhabe Irans in der Zukunft gerichtet war. Vgl. FRUS 61-63, XVIII, S. 98 n3; vgl. Komer Memo, 14.9.62, ebd., S. 97f.; Tehran \#394, 13.9.62, CF 60-63, 788.56300/9-1362; Hart Memo, 4.1.60, 788.56300/1-460; Irwin II Letter, 9.12.55, CF 55-59, 788.5612/12-959; Hilsman Memo [INR Research Memorandum RSB-160], 21.9.62, ,Iran, 1961-1962 [Folder 1 of 2]“, NSF, RWK, Box 424, JFKL; sowohl INR wie auch Komer erwähnten einen möglichen Bezug zu Kuba, ohne eine mögliche sowjetische Stationierung von Nuklearwaffen auf der Karibikinsel in Erwägung zu ziehen. KuZICHKIN, Inside the KGB, S.215ff., behauptet ein von Chruschtschow persönlich angeordnetes Attentat gegen den Schah 1961. Die in einem Auto entlang der täglichen Route des Schahs deponierte Bombe habe aber nicht gezündet. Die Behauptung erscheint sehr zweifelhaft, zumal Kuzichkin als Überläufer wohl ein Interesse an derlei Übertreibungen der sowjetischen Aktivitäten hatte

${ }_{99}$ Vgl. Tehran \#428, 21.9.62; Tehran \#429, 21.9.62, ,Iran General 9/12/62-9/23/62‘, NSF, Countries, Box 116, JFKL; ein umfassendes Rapprochement mit Moskau würde einen „very heavy psychological blow to us“" sowie einen ,significant shift in the balance of power" bedeuten, wie ein Contingency Paper feststellte. Vgl. Bowling Memo, 8.10.62, ,Office Memoranda, 1962', Box 4; Iran Contingency Paper, 5.6.62, enclos. to Rockwell Memo, 5.6.62, ,3-A/1. Military Contingency Planning‘, Box 6; o.A., ,Iran Pledges to USSR', o.D., , 16. USSR-Iran Relations; 1962‘, NEA, GTI: RIAD, 1958-63, Box 8; für das Kalkül vgl. Bowling Hypothetical Memo, 27.4.61, ,20 Task Force on Iran“, Box 4, ebd.; gemäß westdeutschen Quellen waren die USA, nicht unzufrieden“ mit der Entwicklung. Siehe Botschaft Washington an AA, 6.12.62, B 12/1010, PAAA; ein US-Offizieller erinnerte sich später, dass die Versuche des Schahs, seine „Soviet card“" zu spielen, im Grunde nie wirklich ernst genommen wurden. Vgl. Theodore L. Eliot OHI, 29.7.86, OHIC, S. I-46; unkundig die Einschätzung von SuMMITT, White Revolution, S. 568; ähnlich POLLACK, Persian Puzzle, S. 81f.; Entstehungsgeschichte und Verhandlungen ausführlich in ALVANDI, Missile Base Pledge.

${ }^{100}$ Zumindest informierte Mohammad Rezā in einer Rede in Täbris im Oktober 1962 die iranische Öffentlichkeit über die geplante Truppenreduktion, was seitens der Botschaft als positives Signal gewertet wurde. Siehe Tehran \#A-187, 10.10.62, 788.5/10-1062, CF 60-63.
} 
Politik durch die Kennedy-Administration erstaunlich. Tatsächlich ist in diesem Zeitraum ein Wendepunkt im US-Ansatz gegenüber Iran zu verorten, der - bei aller vermeintlichen Ähnlichkeit zur Strategie der späten Eisenhower-Administration - im Kern eine grundsätzliche Re-Orientierung weg vom Leitbild der Permanenten Intervention signalisierte.

\section{2. Reformistischer Interventionismus und strategische Interessen der USA:}

Hintergründe und Ursachen des Strategiewechsels

Bevor in Kapitel VI auf die graduelle Abkehr von den die US-amerikanische Iranpolitik im Grunde seit den frühen 1950er Jahren prägenden Konzepten eingegangen wird, soll zuerst eine Einschätzung des gescheiterten Experiments reformistischer Intervention in der Kennedy-Ära unternommen werden. Was im Nachhinein als episodenhafte Marginalie oder nur kurzlebiges Intermezzo erscheint, stellt von der Warte der Gesamtheit der amerikanischen Iran- bzw. Nahostpolitik aus betrachtet, bzw. mit Blick auf die Herangehensweise gegenüber der sogenannten Dritten Welt im Allgemeinen, einen bemerkenswerten Kontrapunkt zu den - im Übrigen auch während dieser Phase durchgehend wahrnehmbaren - etablierten Kontinuitäten amerikanischer Grand Strategy dar. Sämtliche Schwächen und Widersprüche der bislang verfolgten Strategie konsequent wie rücksichtslos identifizierend und eine (trotz aller zugrunde liegenden Vorsicht und nicht $\mathrm{zu}$ vermeidenden Kompromissen in der spezifischen Formulierung) alternative Richtung für die zukünftige US-Politik gegenüber Iran vorgebend, lag hier die Chance einer fundamentalen Kehrtwende: In ihrer Gesamtheit umgesetzt, wäre die neue Strategie auf eine Transformation der inneren Verfasstheit des Klientelstaates wie auch auf eine qualitative Neugestaltung der iranisch-amerikanischen Allianz im Kontext des globalen Kalten Krieges hinausgelaufen. In der Retrospektive erscheint das „AminiExperiment" als Gelegenheit für eine historische Korrektur der langfristig so fatalen Entscheidung zugunsten des Sturzes Mosaddeġs. Ein von amerikanischer Seite ermöglichter Erfolg Aminis hätte durchaus die Richtung der bilateralen Beziehungen verändern können - es sei letztlich eine „missed opportunity of rather sizable proportions" gewesen, so die postrevolutionäre Einschätzung eines der besten Irankenner im State Department. Kontrafaktische Spekulation über den weiteren Verlauf der iranischen Geschichte und der Beziehung zur westlichen Vormacht sind ebenso verlockend wie gegenstandslos. ${ }^{101}$

Wenngleich über die historischen Folgen einer tatsächlich erfolgreich umgesetzten Kehrtwende

${ }_{101}$ Zitat aus William G. Miller OHI, 25.3.85, Transcript 5, S. 18, IOHP; ein Beispiel für solch kontrafaktische Spekulation findet sich u.a. in SMITH, America s Mission, S. 212: „But what of the missed opportunities of the early $1950 \mathrm{~s},[\ldots]$, or in the early 1960 s when, at Washington's urging, the shah initiated a series of reforms that might have transformed him into a constitutional monarch? By its support for the shah, the United States got twenty-five years of good relations with Iran. In the fifteen years since he fell, Washington has felt the full blast of outraged Iranian nationalism."; ähnlich RAMAZANI, Patterns of Influence, S. 77; für eine breiter angelegte Spekulation über den Einfluss der USA auf die politische Verfasstheit Irans vgl. GooDE, A Liberal Iran. 
in der US-Politik nur gemutmaßt werden kann, so bleibt doch wenigstens die Möglichkeit, die Gründe für das Scheitern des Versuchs wissenschaftlich zu ergründen. Daher sollen in diesem Unterkapitel die unterschiedlichen in der Forschungsliteratur zu findenden Erklärungsansätze für die Aufgabe der Politik des reformistischen Interventionismus systematisch untersucht werden: (1) die bürokratische These einer bewussten Manipulation der reformistischen Ansätze durch Teile der Regierungsbürokratie; (2) die These einer erfolgreichen „Ko-optation“ der New Frontier durch einen nun Reformen gegenüber aufgeschlossenen Mohammad Rezā; (3) die verwandte, aber breiter angelegte peri-zentrische These einer Selbstüberschätzung der Einflussmöglichkeiten durch die Administration und des Scheiterns des reformistischen Programms am Widerstand der lokalen Akteure und ihrer erfolgreichen Manipulation metropolitaner Absichten mittels der Drohung außenpolitischer Neuorientierung; (4) die Vorstellung, eine gravierende Verschlechterung der regionalen Position der USA aufgrund des Scheiterns ihrer Disengagement-Strategie habe eine Rückbesinnung auf die bewährten Verbündeten wie den Schah induziert und, in eine ähnliche Richtung zielend, die (5) alternative regionale These, wonach nicht unmittelbare Krisenerscheinungen, sondern eine langfristig angelegte Anpassung der Grand Strategy an die erwartete Transformation der Golfregion infolge der Auflösung des anglo-amerikanischen Kondominiums eine strategische Neubewertung und Aufwertung Irans impliziert habe, was letztlich eine Aufgabe innenpolitischer Experimente vonnöten gemacht habe, sowie zuletzt (6) die These einer global induzierten Neubewertung Irans im Kontext der globalen militärstrategischen Wende hin zur Flexible Response mit demselben Effekt einer Abnahme der Risikobereitschaft bezüglich innerer Machtverschiebungen.

Tatsächlich lassen sich sämtliche sechs Thesen empirisch belegen, doch können gleichzeitig keine der Thesen und auch keine Kombination einiger dieser Thesen eine hinreichende Erklärung für die Aufgabe der reformistischen Intervention bieten. Ich vertrete daher die Auffassung, dass sich diese strategische Wende letztlich nicht allein im Gesamtzusammenhang der bilateralen Beziehungen zu Iran erklären lässt. Vielmehr bestimme ich als den eigentlichen Auslöser für die politische Kehrtwende in Iran eine - wenngleich bislang nicht von der Kennedy-Forschung identifizierte - Anpassung der Grand Strategy gegenüber der globalen Peripherie, grob datiert auf die Jahreswende 1961/62 und öffentlich verkündet mit der Rede des Präsidenten am 16. November 1961 in Seattle. Im Kern wurde die US-Politik gegenüber der Peripherie neu kalibriert, weg von der Betonung innerer Reform und Entwicklung und zurück auf Stabilisierung und Repression. ${ }^{102}$ ${ }^{102}$ An dieser Stelle seien noch jüngere Interpretationen genannt, die in keine der hier vorgeschlagenen Kategorien
passen. Victor Nemchenok argumentiert, der reformistische Interventionismus ab 1961 sei im Kern nur eine
Fortsetzung der Eisenhower'schen Stabilisierungspolitik mit anderen Mitteln gewesen sei - keine der Fraktionen
innerhalb der Administration habe eine politische Transformation in irgendeine Form angestrebt. Es habe
demzufolge auch keinen bürokratischen Konflikt über die Iranpolitik innerhalb der Administration gegeben. Vgl.
NEMCHENOK, Search of Stability; die fehlerreiche Analyse und etwas willkürliche Interpretation Nemchenoks
erklärt sich in erste Linie aus der Quellenarmut der Untersuchung, ohne Rückgriff auf Dokumente aus der Kennedy
Library bzw. den National Archives. Noch zweifelhafter ist die idiosynkratische Interpretation von WARNE,
Psychoanalyzing Iran; Warne sieht die schnelle Aufgabe ernsthafter Reformgedanken als Folge eines in der 
Wie in den drei vorangegangenen Kapiteln detailliert geschildert, war die Task Force-Politik in der Frühphase der Kennedy-Administration eine ernstgemeinte und vonseiten des Weißen Hauses mit Nachdruck gegenüber State Department und anderen Behörden durchgesetzte strategische Neuausrichtung. Allerdings muss betont werden, dass es in der Kennedy-Ära nur wenige Beispiele gab, in denen die zwischenbehördlichen Auseinandersetzungen mit einer solchen Schärfe geführt wurden wie im Falle der reformistischen Intervention im Iran. Die wiederholten Versuche des State Department, und insbesondere der US-Botschaft in Teheran unter Holmes‘ Führung, die Vorgaben des Weißen Hauses zu ignorieren bzw. die Task ForcePolitik zu verwässern, haben fraglos zu dem Scheiten des Gesamtkonzepts beigetragen. Die (1) bürokratische Interpretation der Kennedy'schen Iranpolitik geht somit auch davon aus, dass der Widerstand seitens der Ausführungsorgane und insbesondere seitens State Department und Militär hauptverantwortlich für deren Scheitern waren. In seiner grundlegenden Untersuchung der Kennedy-Politik hat James Goode bereits wiederholt auf die Bedeutung des bürokratischen Widerstands hingewiesen. Mark Gasiorowski sieht die amerikanische Politik infolge der Opposition innerhalb der Administration ,nahezu paralysiert“. Roby Barrett zufolge hatte Botschafter Holmes sogar ,eigenhändig“ die Rückkehr zur tradierten Iranpolitik Eisenhowers durchgesetzt. ${ }^{103}$

Die bürokratische These im Sinne von Arthur Schlesinger Jr., also das Scheitern der interventionistischen Reform im Iran vorrangig auf einen Sieg der ,permanenten“ über die „politische Regierung“ zurückzuführen, ist verlockend und erscheint dem Historiker intuitiv als der überzeugendste Erklärungsversuch, nicht zuletzt da bei intensiver Beschäftigung mit dem für die Iranpolitik bedeutsamen Archivmaterial das Fortdauern und die Intensität der

\footnotetext{
Administration dominierenden negativ konnotierten Iranbilds. Ältere orientalistische und rassistische Vorstellungen hätten sich Mitte des 20. Jahrhunderts in pseudowissenschaftlichen Psychologisierungsversuchen erhalten, die sich in der Darstellung des iranischen Nationalcharakters und der Iraner als defizitär, deviant bzw. psychisch krank widerspiegelten. Dies habe letztlich in der fortgesetzten Unterstützung einer Diktatur resultiert. Abgesehen von der fehlerhaften Darstellung der Kennedy'schen Iranpolitik und dem Fehlen jeglicher Archivquellen ist an dieser Interpretation zu kritisieren, dass einige wenige selektive Quellenzitate essentialisiert und generalisiert werden und Warne ein ebenso einseitig monolithisches Bild der zeitgenössischen US-Offiziellen zeichnet, wie er es zugleich deren Wahrnehmung Irans unterstellt. Ohne Frage existierten ethnozentrische und auch rassistische Vorstellungen weiter, wenngleich diese in internen Debatten nicht mehr als seriöse Argumente taugten und daher kaum in den Quellen auszumachen sind. Neben dem unreflektierten Textualismus' Warnes fällt aber zudem dessen eigene stark ethnozentrische Sichtweise auf, die in den nicht-westlichen Akteuren letztlich nur Objekte einer quasi omnipotenten westlich-amerikanischen Politik sieht. Entgegen der Behauptungen Warnes sah Washington Iran durchaus auf einem Weg der Transformation nach westlichem Vorbild, mündend in einer konvergenten Moderne nach amerikanischem Modell. Die Unterstützung für den sozialen, langfristig implizit auch für den politischen Wandel in Iran demonstriert aber, dass die rassistischen Stereotypen, die Warne ausgemacht haben will, nicht in dieser Schärfe vorhanden und schon gar nicht handlungsleitend waren. Auch die Folgezeit mit der Wahrnehmung Irans als modernistischem Vorbild widerspricht der Permanenz solcher ideellen Determinanten. COLliER, Prevent a Revolution, datiert die Aufgabe des reformistischen Interventionismus korrekt, sieht diese aber mit der Sorge vor Instabilität und einer Hinwendung des Schahs zu Moskau begründet und stellt darüber hinaus eine Reihe von empirisch nicht belegbaren Thesen auf, z.B. den bewussten Sturz Aminis durch die USA.

${ }_{103}$ Goode, Reforming Iran, S. 17, 28; Mark Gasiorowski in einer Email an den Autor vom 11.6.2007; BARRETT, Greater Middle East, S. 233f., 241; seit dem Erscheinen des Goode-Artikels ist die Betonung der bürokratischen Dimension der locus classicus der geschichtswissenschaftlichen Interpretation der Vorgänge. Geradezu absurd ist die Behauptung von SuMMITT über das Fehlen dieser Perspektive in der bisherigen Forschung. Siehe dies., White Revolution, S. $561 n 1$.
} 
Auseinandersetzungen innerhalb der Administration geradezu augenfällig sind. ${ }^{104}$ Bei genauerer Betrachtung aber zeigt sich, dass die Abfolge der Ereignisse und der graduelle Wandel im Ansatz der Kennedy-Administration nur bedingt auf den bürokratischen Widerstand zurückgeführt werden können. Die Sprache des Task Force Report vom Mai 1961 widerspiegelte weitgehend unverwässert die reformistisch intendierte Interventionsbereitschaft in der Frühphase der New Frontier. NSAM N 67 vom August 1961 hatte den Druck auf State Department und andere Behörden weiterhin aufrechterhalten. Bereits kurz danach aber erlahmte der Reformeifer der Kennedy-Administration spürbar, trotz der zu dieser Zeit eher einsichtigen Haltung NEAs und der (erfolgreichen) anglo-amerikanischen Intervention zugunsten Aminis im Oktober. Im Grunde verlor der reformistische Impuls genau in dem Zeitraum an Kraft, in welchem der bürokratische Widerstand weitestgehend gebrochen schien, abgesehen von der grundsätzlichen Haltung der allermeisten hohen Militärs. ${ }^{105}$ Somit entsteht eher der Eindruck, dass sich bereits ab dem Herbst 1961 schrittweise auch innerhalb des Weißen Hauses Zweifel über die reformistischen Interventionen im Allgemeinen mehrten. Angesichts des fortdauernden Reformeifers Robert Komers im NSC-Stab und seiner Verbündeten in anderen Behörden dauert zwar in den Akten der scharfe Konflikt mit State Department und vor allem mit der Botschaft in Teheran fort. Nach dem Herbst 1961 drehen sich die Streitigkeiten allerdings vorwiegend um kleinere Details, meist im MAP-Programm, kaum noch um die großen reformistischen Ideen des Frühlings wie z.B. den (klandestinen) Aufbau einer „politischen Synthese“ alternativ zur Schah-Diktatur - bezeichnenderweise wurde Anfang 1962 die Antwort des State Department auf NSAM N67 weitgehend kritiklos hingenommen, obwohl diese im Kern eine Aufgabe der Task Force-Politik implizierte. Hätte die präsidentielle Rückendeckung in gleichem Maße wie im Frühling 1961 bestanden, ist davon auszugehen, dass sich die Reformisten gegen die Haltung von Foggy Bottom entschlossener und auch erfolgreich zur Wehr gesetzt hätten.

Zudem muss darauf verwiesen werden, dass die Positionen nicht so gegensätzlich waren, wie es die Akten ex post facto suggerieren. Die Extrempositionen eines Kenneth Hansen und eines Julius Holmes waren in der Tat unvereinbar - das Gros der Einstellungen hinsichtlich der wünschenswerten US-Haltung gegenüber Iran innerhalb der Administration und den relevanten Behörden aber befand sich irgendwo zwischen diesen beiden Polen. ${ }^{106}$ Den ,extremen“ (wenn auch in diesem Fall eher altruistisch motivierten) Interventionismus eines Hansen teilte kaum jemand im Weißen Haus. Anders als Holmes waren aber die meisten Offiziellen innerhalb des State Department durchaus vom dringlichen Reformbedarf in Iran überzeugt. Die Iranexperten

\footnotetext{
${ }^{104}$ Siehe Schlesinger Jr., oben S. 292.

${ }^{105}$ Allerdings muss bezüglich des militärischen Widerstands gerade seitens der JCS auf den schwindenden Einfluss der militärischen Führung auf vorwiegend politische Entscheidungen verwiesen werden. Siehe KAPLAN/LANDA/DREA, History of the Office of the Secretary of Defense: V, S. 297; im Kern hatten die Umstände, die zur gemeinsamen anglo-amerikanischen Intervention vom Oktober 1961 und der vorübergehenden ,Rettung“" Aminis geführt hatten, eigentlich exakt jene Problematiken exemplifiziert, die im Task Force-Report als Hauptargument für eine Neuorientierung der US-Politik genannt worden waren.

${ }^{106}$ Dem Autor der vorliegenden Studie ist bewusst, dass Vertreter des „,klassischen“ Ansatzes der bureaucratic politics in dieser Feststellung keine Falsifizierung des eigenen theoretischen Zugangs sehen würden.
} 
in NEA stimmten mit Bob Komer zudem überein in der Analyse des Problems. Die bisweilen während der bürokratischen „Infights“ zur Schau gestellte Gelassenheit bezüglich der weiteren Entwicklung in Iran entsprach häufig nicht den eigenen Überzeugungen. Exemplarisch steht dafür ein internes Memorandum des Iran Desk Officer in GTI, John Bowling, ein durchgehend entschlossener Gegner des zeitweilig vom Weißen Haus präferierten reformistischen Interventionismus. Verfasst im November 1961, also gerade zu der Zeit, als das State Department sich der verbliebenen Reste der Task Force-Politik entledigte, setzte sich Bowling mit der ,Real Policy Question on Iran“, nämlich der grundsätzlichen US-Haltung gegenüber dem Schah-Regime, auseinander. ${ }^{107}$ Für Bowling ging es hier um keine einmalige Entscheidung, sondern um die fortgesetzte und immer wieder von Neuem zu entscheidende Grundausrichtung der US-Politik in Iran. Dabei konstatierte er in entwaffnender Offenheit, in Iran wirkten ,deep and long-term sociological and psychological trends“, die ,[...] on balance inimical to our national security interests" seien. Diese Trends, so die Analyse, würden zwangsläufig auf einen Machtwechsel in Iran hinauslaufen, von der gegenwärtigen Dominanz des Hofs hin zur Übernahme der Kontrolle durch die nationalistische Opposition, den „Mosadeqists“. Die Idee, mit einer gemäßigten und gestärkten Regierung unter einem unabhängigen Premierminister, das „Amini-Experiment“, diese Opposition mittelfristig mit der herrschenden Elite zu versöhnen bzw. diese ins politische System zu re-integrieren, sei gescheitert. ${ }^{108}$

Neben der Unversöhnlichkeit der nationalistischen Opposition läge dies allerdings in erster Line an der Persönlichkeit Mohammad Rezās. Der Schah sei über die Jahre nicht gereift, sei trotz seiner Intelligenz zu misstrauisch, eitel, und letztlich zu anfällig für Schmeicheleien, um in der Lage zu „,moderner politischer Führung“ zu sein: „On the day before he is overthrown, the Shah will undoubtedly be primarily concerned over the absence of esoteric military equipment in his Army or over what some obscure French newspaper has to say about him." ${ }^{109}$ Somit unterstütze man ein „,doomed regime“ - Iran sei „politically and psychologically sick unto death". Früher oder später würde eine Regierung der Nationalen Front die Macht übernehmen, mit den bekannten Konsequenzen des Zusammenbruchs der CENTO-Allianz, der Aufkündigung des Ölkonsortiums, dem Abzug der Militärberater, dem Verlust Irans als

\footnotetext{
${ }^{107}$ Bowling definierte die Kernfrage wie folgt: „, The question is whether or not the United States should continue to identify itself with and support the monarchical regime in Iran or whether it should allow or even assist in the transfer of political power to the group loosely known as Nationalists.“; Bowling Memo, ,The Real Policy Question on Iran', 2.11.61, ,16. U.S.-Iran Relations‘, NEA, GTI: RIAD, 1958-63, Box 3.

${ }_{108}$ Bowling zufolge gab es auch keine politische Alternative ,,unless and until the political factors in Iran change radically.“; Amini sei die perfekte Wahl für diese Rolle gewesen: „Amini was uniquely situated equidistant between the conservatives, the Mosadeqists, and the Shah; still he was unable to form the centrist coalition which is the only alternative solution other than some now unseen totalitarian demagogic force."; die ,centrist coalition“" ist wohl eine Referenz auf die „broad political synthesis“ des Task Force Report. Alternative Führungspersönlichkeiten sah Bowling nicht: Die konservative Elite sei ,,hopelessly short-sighted“, im Militär fänden sich keine Leute von Format: ,[...] its would-be politicians are timeservers, fools, or brutes.“; zudem müsste auch ein militärischer Führer nach einer Machtübernahme entweder die politische Linie des Schahs (ohne das an die monarchische Institution weiterhin gebundene Vertrauen) fortführen oder auf die Forderungen der städtischen Opposition eingehen (,which would make him a Mosadeqist in uniform“).

109 Man beachte die Unvereinbarkeit dieser Einschätzung des Herrschers mit der ,offiziellen“ GTI-NEA-Position in den wichtigen Bowling-Memoranden vom März 1961.
} 
„,intelligence base“, sowie allgemein wachsendem sowjetischen Einfluss. Je länger die USA mit dem Schah-Regime identifiziert würden, desto wahrscheinlicher sei in der Folge die antiwestliche bzw. anti-amerikanische Einstellung der Nachfolgeregierung. ${ }^{110}$ Anschließend offerierte Bowling noch eine Einschätzung der wahrscheinlichen zukünftigen Entwicklung:

(A) Chances of overthrow of the Shah within 5 years $\quad 70 \%$

(B) Chances of overthrow of the Shah within 10 years $\quad 90 \%$

(C) Chances of Shah going into arms of USSR within $\quad 10 \%$ 5 years if he remains in power

(D) Chances of communist control of Iran within 5 years $\quad 40 \%$ in case of revolution now without U.S. participation
Chances of communist control of Iran within 5 years

(E) Chances of communist control of Iran within 5 years
in case of revolution now with U.S. participation

(F) Yearly increase in chances of communist control of 2,5\% Iran within 5 years under (D) or (E) above as long as the Shah remains in power ${ }^{111}$

Bowlings Memorandum vom November 1961 ist zweifellos eines der erstaunlichsten Dokumente im Zusammenhang mit US-amerikanischer Iranpolitik. Zwar unterschätzte er die Überlebensfähigkeit des Regimes und übersah die (zu dieser Zeit allerdings noch von keinem Beobachter vorhergesehene) Entstehung einer neuen politischen Strömung, die auf einer revolutionären Re-interpretation der schiitischen Glaubensvorstellungen basierte. ${ }^{112}$ Dennoch sagte er die langfristige Entwicklung vorher, insbesondere die letztendliche Bedeutungslosigkeit erfolgreicher wirtschaftlicher Modernisierung bei gleichzeitigem Fortdauern politischer Repression. Trotz seiner pessimistischen Einschätzung für die Überlebenschancen der Pahlawi-Monarchie plädierte Bowling dennoch für eine Fortsetzung der bisherigen Politik, wenn auch die Notwendigkeit betonend, jederzeit für einen Politikwechsel bereit sein zu müssen. ${ }^{113}$ Interessant ist darüber hinaus die Feststellung Bowlings, dass man eigentlich eine solche Einschätzung der Lage dem NSC vorlegen müsse, aufgrund von früheren Erfahrungen aber davon Abstand nehmen solle. Dies würde zwangsläufig in „desperate and ill-advised measures“ resultieren, welche die

\footnotetext{
${ }^{110}$ Angesichts der jüngeren Geschichte Irans müsste selbst eine mit US-Unterstützung an die Macht gelangte nationalistische Regierung anti-amerikanische Maßnahmen durchsetzen, um Glaubwürdigkeit zu erlangen - somit wäre eine effektive Schädigung von basalen US-Interessen unvermeidbar. Vgl Bowling Memo, 2.11.61, oben Anm. 107.

${ }^{111}$ Ebd.

${ }^{112}$ Bowlings Einschätzungen der kommunistischen Gefahr wurde im Übrigen von den meisten US-Offiziellen nicht geteilt. Zwar rechnete auch die CIA für den Fall eines Umsturzes mit einem erneuten Wachstum einer kommunistischen Partei, angesichts der bestehenden Schwäche der Organisation zu diesem Zeitpunkt aber nicht mit einer auch nur mittelfristigen Gefahr einer kommunistischen Machtübernahme. Hier spiegelte sich eher der vehemente Antikommunismus Bowlings.

${ }_{113}$ Angesichts seiner Vorhersagen war dies ein Widerspruch, der auch Bowling selbst bewusst war: ,On balance, the decision adds up to a question of whether one maximizes one's long-term possibilities while accepting immediate losses, or maximizes one's short-term possibilities at the expense of greater long-term dangers. My personal preference would be to continue the latter policy, not out of a feeling of 'apres moi le deluge $\{$ sic $\}$ ', but out of a belief that the future may contain new possibilities which we cannot foresee at present, and out of the belief that, strange as it seems, supporting the Shah is more ethical from a Western point of view than a Machiavellian hastening of a so-called 'democratic' revolutionary trend which will actually bring about a decline in the real liberty and well-being of the great masses of the Iranian people, no matter how much satisfaction it may bring to secondary school and college graduates there."; Letzteres ist im Kern eine fast schon klassische Zusammenfassung einer konservativen und anti-revolutionären Weltsicht.
} 
Wahrscheinlichkeit einer Verschlechterung der Gesamtlage nur erhöhen würden. Hier spielt der Widerwille gegen jede Einmischung der (aus Bowlings Sicht ohnehin unqualifizierten) Berater aus dem Weißen Haus in ureigene Kompetenzen des State Department durchaus eine Rolle.

Insgesamt aber illustriert das Bowling-Memorandum die Schwäche des bürokratischen Arguments. Seine Analyse der internen Lage in Iran korrespondierte durchaus mit der anfangs im Weißen Haus dominierenden Sichtweise. Gemeinsam mit dem reformistischen Interventionismus gegenüber aufgeschlossenen Offiziellen wie dem Assistant Secretary Phillips Talbot und einigen denkbaren Neubesetzungen, wie wiederholt von Komer und seinesgleichen gefordert, wäre eine konsequente Fortsetzung der Task Force-Politik auch gegen den Widerstand des State Department fraglos möglich gewesen. Bürokratische Gegenwehr ist somit keine hinreichende Erklärung für die Aufgabe des reformistischen Interventionismus seitens der Kennedy-Administration.

Eine alternative, ebenfalls häufig in der Literatur zu findende These besagt, dass es Schah Mohammad Rezā gelungen sei, die New Frontier im Besonderen und die USA im Allgemeinen von seiner herausgehobenen Eignung und Regierungskompetenz zu überzeugen. Dieser (2) Ko-Optationsthese zufolge stellten die USA ihre Reformbemühungen aufgrund der Überzeugungsarbeit des Schahs ein. So resümiert James Goode: „The shah subsequently convinced President Kennedy that he, too, favored reform, that he represented tomorrow rather than yesterday, and the Kennedy administration belatedly endorsed his regime."114 In eine ähnliche Richtung zielt die Interpretation von Tony Smith, der die Hinnahme der Beseitigung Aminis mit der amerikanischen Sichtweise begründet, ,[...] that viable reform was still under way because the monarch asserted his continued commitment to social and economic reform." $" 115$

Nicht von der Hand zu weisen ist die Akzeptanz Mohammad Rezās als weitsichtiger und prowestlicher Reformer, hier beginnend in den frühen 1960er Jahren, und die Marginalisierung jedweder Kritik an der Pahlawi-Diktatur in dieser Zeit, sowohl innerhalb der USAdministrationen wie auch zunehmend in der amerikanischen Öffentlichkeit. Zur Erklärung des Scheiterns des Amini-Experiments und der reformistischen Intervention taugt die These einer erfolgreichen Ko-Optation durch den Schah allerdings nicht. Ein Kausalzusammenhang ist bereits chronologisch ausgeschlossen, da die eigentliche ,persönliche“ Reformaktivität des Schahs im Rahmen der Weißen Revolution erst Ende 1962 mit Nachdruck einsetzt, und somit

\footnotetext{
${ }_{114}$ GoodE, Reforming Iran, S. 13; tatsächlich verbindet Goode in seiner Argumentation Bürokratie- und KoOptationsthese: „U.S. support for the shah's White Revolution was thus the by-product of a bureaucratic compromise between those who would have preferred a middle-class planner to rule Iran and those who preferred the traditional elite."; ebd., S. 24; ähnlich CASTIGLIONI, Start a Revolution, S. $111 \mathrm{ff}$.

115 Smith, America's Mission, S. 219f.; vgl. auch ebd., S. 227f., 255f.; ähnlich SuMmitT, White Revolution, S. 573.
} 
einige Monate nach dem Rücktritt Aminis und fast ein Jahr nach dem Erlahmen des amerikanischen Reformeifers. Der reformistische Interventionismus der New Frontier wurde somit nicht zugunsten eines indigenen Reformismus des Hofes aufgegeben. Tatsächlich finden sich die Lobpreisungen des Schahs, die gerade für die 1970er Jahre so kennzeichnend werden sollten, zu dieser Zeit noch nicht. Innerhalb der Bürokratie herrschte eine sehr kritische Einschätzung der Führungsfähigkeit wie auch der persönlichen Charaktereigenschaften des Schahs vor, wie sie sich auch im eben diskutierten Bowling-Memorandum wiederfand. ${ }^{116}$ Das Pahlavists-Netzwerk in den USA versuchte unentwegt, das Image des Schahs in den USA weiter zu verbessern, dabei gerade seine Reformbemühungen herausstreichend. Beispielhaft für diese Bemühungen ist die Veröffentlichung der englischsprachigen Ausgabe seines autobiographisch-programmatischen ersten Buches Mission for My Country (1960). Das Buch richtete sich mit seiner demonstrativen Bejahung westlicher Modernitätsvorstellungen und einzelnen Kapiteln zu Entwicklungszielen und Frauenemanzipation unzweifelhaft an eine westliche Leserschaft. Mitunter krude wirkte die Verschleierung der autoritären Regierungsweise. So schrieb Mohammad Rezā unter anderem über seine „[...] three-fold idea of democracy, and the steps we have taken to help make true democracy come alive here [...]“. ${ }^{117}$ Derlei Versuchen, den Schah als Reformer und Demokraten zu portraitieren, waren aber Anfang der 1960er Jahre nur geringer Erfolg beschienen. Der ehemalige Leiter von ARMISH-MAAG ab 1971 erinnerte sich später, wie ihm vor seiner Abreise nach Iran jeder Gesprächspartner in Washington versichert habe, ,[...] what a brilliant man the Shah of Iran was, just the smartest man that ever lived.“ " 118 Zehn Jahre zuvor wäre eine solche Einschätzung der Qualitäten des Monarchen in der Tat noch undenkbar gewesen.

Im Grunde verwandt mit der Ko-Optationsthese ist die (3) peri-zentrische Interpretation. Aus peri-zentrischer Perspektive erklären sich die vielen Rückschläge der Supermächte nicht aufgrund gelungener Manipulation der Metropole durch schwache, aber in spezifischen Fällen persuasive Akteure an der Peripherie, sondern aus der grundsätzlichen Autonomie und trotz aller Asymmetrien gegenüber dem Hegemon auch beträchtlichen Handlungsfreiheit der Junior Powers. ${ }^{119}$ Dieser Sichtweise zufolge erklärt sich das Scheitern des Kennedy-Reformismus aus den begrenzten Einflussmöglichkeiten der USA in Iran und den erfolgreichen Anstrengungen

\footnotetext{
${ }^{116}$ Negative Einschätzungen des Schahs sind zahlreich. So stellte eine grundlegende Studie des State Department fest, dass man , [...] have no reason to feel any confidence in the Shah's ability to tackle realistically the long-range problems of Iran“. Zit. aus. ,NEA Study of Possible U.S. Actions RE the Long-Term Political Situation in Iran“, 10.8.61, encl. to Kerr Memo, 11.6.61, ,20 Task Force on Iran', NEA, GTI: RIAD, 1958-63, Box 3; selbst der konsequenteste Unterstützer der monarchischen Ambitionen, Botschafter Holmes, äußerte sich zuweilen sehr negativ über dessen Qualitäten. Siehe z.B. Tehran \#186, 16.10.61, oben, S. 282, Anm. 179.

${ }^{117}$ Vgl. PahlaVi, Mission for My Country, S. 13; zum Hintergrund AFKHAMI, Life and Times, S. 220-6; zur sich ändernden Haltung der Presse vgl. DoRman/FArhang, U.S. Press and Iran, S. 98-115; absurd die Behauptung Summitts, dem Schah sei es gelungen, Washington zu überzeugen, die iranische Opposition sei ein Werkzeug der Sowjets. Siehe SuMMITT, White Revolution, S. 573.

${ }^{118}$ Ellis Williamson OHI, 13.4.88, S. I-2; Ellis gestand zudem ein, dass er nach einigen Treffen mit dem Monarchen in der Tat zu dem Schluss kam, ,[...] that he was probably one of the most brilliant men I had ever met.“

${ }_{119}$ Aufgewertet zu einer eigenständigen Interpretationsschule der Cold War History durch SMITH, Pericentric Framework; eine Erklärung US-amerikanischen Interventionen mit dem Einfluss „kleiner“ Akteure als causa causans bei KARABELL, Architects of Intervention, S. 4-15.
} 
des Hofs, die Reformbemühungen der Kennedy-Administration zu unterlaufen. Roby Barrett zufolge hatte die New Frontier die Widerstandsfähigkeit des Schahs und dessen Kontrolle über das politische System in Iran unter- und zugleich den eigenen Einfluss überschätzt: „In reality, the United States never had any real control over events in Iran." ${ }^{\text {120 }}$

Zweifelsohne sind die in frühen Untersuchungen häufig anzutreffenden holzschnittartigen Annahmen über Washingtons Quasi-Omnipotenz an der Peripherie abzulehnen. Zudem steht außer Frage, dass Mohammad Rezā das wichtigste Machtzentrum in Iran dieser Zeit darstellte und die zeitgenössischen Vorstellungen über die amerikanischen Einflussmöglichkeiten überzogen waren. Der Schah selbst stellte diesbezüglich gegenüber Dean Rusk fest: ,[...] we are not your stooges." ${ }^{121}$ Dennoch muss das entgegengesetzte Extrem, eine Überbetonung der Handlungsfreiheit peripherer Akteure ebenfalls vermieden werden. Nicht von ungefähr wurde seitens der iranischen Führung wie auch der Opposition im Land jede reale wie scheinbare Wendung in der amerikanischen Politik mit größter Aufmerksamkeit registriert und das Verhältnis zu den USA als die mit Abstand wichtigste auswärtige Beziehung angesehen. Vergleichbare Fälle wie z.B. Südvietnam zeigen zudem, dass die USA durchaus in der Lage waren, eine missliebige Führung zu beseitigen, wenn sie nur den politischen Willen dafür aufbrachten. So gab sich auch Bob Komer überzeugt, dass man in der Lage sei den Schah zu stürzen ,[...] simply by withdrawing aid and letting nature take its course“. ${ }^{122}$

Die ersten Monate des Amini-Experiments belegen zudem die Gestaltungsmöglichkeiten amerikanischer Politik in Iran auch gegen indigene Widerstände, solange solche Bemühungen auf starke präsidentielle Unterstützung bauen konnten. In verbündeten Staaten, anders als z.B. in Kuba, konnte man zudem immer auf den eigenen „strategischen Einfluss“ vertrauen, die weiter bestehenden Kontakte zu wichtigen pro-amerikanischen Netzwerken in Militär, Ministerien, Wirtschaft und zu bedeutenden gesellschaftlichen Akteuren. Bei entsprechendem Willen war es somit möglich, tragfähige Koalitionen zu schmieden und diese offen wie verdeckt zu unterstützen. Seit dem Sturz Mosaddeġs war ein solcher Einfluss der USA im Sinne der Permanenten Intervention in der Tat gegeben. Was die Episode des reformistischen Interventionismus angeht, so wurden Widerstand und Unbehagen des Schahs selbstverständlich wahrgenommen, an der Formulierung eines umfassenden und von Außen massiv gestützten Reformprojekts und seiner beginnenden Implementierung änderte dies jedoch anfangs nichts. Die Haltung des Hofs war fortwährend negativ und kontraproduktiv, konnte allerdings auch erst dann das Amini-Experiment unterlaufen, als die Unterstützung seitens Washingtons

\footnotetext{
${ }^{120}$ BARRETT, Greater Middle East, S. 432 n52; Barrett macht diese Einschätzung an der erfolgreichen Ko-optation des Ministerpräsidenten Amini durch den Schah fest, was seine Dominanz nachdrücklich illustriere. Siehe ebd., S. 223, 229-30; allerdings missversteht Barrett die taktische Unterstützung der militärischen Wünsche des Schahs durch Amini als Unterwerfung und ignoriert zudem sämtliche Belege für die lange Zeit bemerkenswerte Handlungsautonomie des Premiers. Plausibler erscheint, dass Aminis stärkere Anlehnung an den Schah ab dem Januar 1962 nicht zuletzt mit der Einstellung amerikanischer Bemühungen, ihn beim Aufbau einer alternativen Machtbasis zu unterstützen, erklärt werden kann.

${ }^{121}$ MemCon, 13.4.62, FRUS 61-63, XVII, S. 611.

122 Siehe oben S. 317, Anm. 82.
} 
aufgegeben worden war. Somit war dieser Widerstand nur ein beitragender Faktor, dient aber für sich allein kaum zur Erklärung der Wende in der amerikanischen Politik. ${ }^{123}$

Die - ohnehin sanften - Drohungen des Schahs, selbst eine neutralistische Wende in der iranischen Außenpolitik einzuleiten und die „sowjetische Karte“ zu spielen, werden ebenfalls des Öfteren als Beleg für die „Tyrannei der Schwachen“ im Kontext der bilateralen Beziehungen zwischen den USA und Iran angeführt. April Summitt sieht den Widerstand des State Department gegen den reformistischen Interventionismus in erster Linie motiviert durch die Angst, der Schah könne sich Moskau annähern. ${ }^{124}$ Ebenso sehen James Bill und Barry Rubin die iranische Deklaration vom September 1962 bezüglich des unilateralen Verzichts auf die Stationierung von Raketen fremder Staaten auf dem eigenen Territorium als Schlüsselereignis. ${ }^{125}$ Zwar erweiterte die Einleitung eines Rapprochements mit Moskau die außenpolitischen Optionen Irans erheblich und war wohl auch innenpolitisch nicht unpopulär - angesichts der weiterhin bestehenden finanziellen wie militärischen Abhängigkeit von den USA war eine äquidistante Positionierung Irans im Kalten Krieg aber nie eine glaubwürdige Option. Im Gegensatz zur späteren Historiographie zu dieser Episode zeichnete sich die zeitgenössische amerikanische Reaktion zu diesen Entwicklungen durch Gelassenheit aus, wohlwissend über den begrenzten Spielraum des Schahs in dieser Sphäre. ${ }^{126}$

Mehr Beachtung in Washington erfuhren eher die subtilen Drohungen des Schahs, die Zusammenarbeit auf der geheimdienstlichen Ebene einzuschränken oder gar einzustellen. Im Gespräch mit Secretary of Defense McNamara im April 1962 wies der Schah dezidiert auf die „valuable information“ hin, die die amerikanischen Abhörstationen und seismischen Messstationen entlang der iranisch-sowjetischen Grenze produzierten. Tatsächlich strebte man in eben dieser Zeit die Verdoppelung der bestehenden Stationen auf insgesamt vier an. Überzeugte reformistische Interventionisten wie z.B. Richter Douglas waren überzeugt, die Zurückhaltung des Weißen Hauses wäre nicht zuletzt auf den Druck der Intelligence Community und auf die Priorisierung der strategischen Interessen gegenüber dem normativen Projekt der USA zurückzuführen: „No one seems strong enough in the State Department to

\footnotetext{
${ }^{123}$ Die von Barrett aufgestellte Behauptung, Inkompetenz und mangelndes Wissen der New Frontier seien dafür verantwortlich, dass man den Widerstand des Schahs nicht vorausgesehen habe, ist irreführend. Tatsächlich war der erwartete Widerstands des Hofes konstitutiver Bestandteil der Task Force-Politik. Vgl. BARRETT, Greater Middle East, S. 223.

${ }^{124}$ Vgl. SuMMITT, White Revolution, S. 568, 574; die Beziehungen zu Moskau hatten sich noch unter Amini wieder erheblich verschlechtert. Vgl. Botschaft Teheran an AA, 9.8.61, B 12/1007, PAAA.

${ }^{125}$ Vgl. BILL, Eagle and Lion, S. 150; RuBIN, Good Intentions, S. 108; BLAKE, Confrontation in Iran, S. 137f., 167-70; BARRETT, Greater Middle East, S. 214, zufolge war es dem Schah sogar gelungen, die KennedyAdministration von der Ernsthaftigkeit seiner angedeuteten Schritte hin zur Nichtpaktgebundenheit zu überzeugen - es habe sich um eine „real option“ gehandelt. Botschafter Holmes benutzte derlei Drohungen des Schahs wiederholt zur Durchsetzung der militärischen Forderungen des Schahs. Vgl. ebd., S. 230; gelassener ALVANDI, Missile Base Pledge.

${ }^{126}$ Vgl. Theodore L. Eliot OHI, 29.7.86, OHIC, S. I-46; zuzustimmen ist GooDE, Reforming Iran, 23, der die Deklaration in erster Linie als innenpolitisch motiviert interpretiert; NEMCHENOK, Search of Stability, S. 346, 358, bei allerdings limitiertem Verständnis der strategischen Gesamtlage, vertritt ebenfalls die Auffassung, der amerikanische Handlungsspielraum wäre durch die neutralistischen Optionen des Schahs stark begrenzt gewesen.
} 
overrule the CIA and the Pentagon."127 Zweifellos war ein Verlust des Standorts Iran für nachrichtendienstliche Operationen nicht wünschenswert. Es darf jedoch nicht übersehen werden, dass ein solch einseitiges Kaprizieren auf den geheimdienstlichen Aspekt an der Grundkonzeption des reformistischen Interventionismus vorbeizielt, die den Totalverlust Irans als Partner bei Ausbleiben notwendiger Reformschritte voraussagte. In diesem Fall wäre die geheimdienstliche Kooperation ebenfalls in Mitleidenschaft gezogen und höchstwahrscheinlich auch beendet worden, wie es dann ja auch 20 Jahre später Realität wurde.

Insgesamt bleibt festzustellen, dass die perizentrische These als Erklärungsansatz nicht zu überzeugen weiß - die Eigenständigkeit des Hofs gegenüber der amerikanischen Vormacht ist in der Tat Folge und nicht Ursache der Aufgabe des reformistischen Interventionismus, wie das folgende Kapitel demonstrieren wird.

Eine über den engeren Beziehungszusammenhang USA-Iran hinausweisende Erklärung für die rasche Aufgabe der Kennedy'schen Reforminitiativen ist die These, die New Frontier sei zur etablierten Anbindung der eigenen Regionalpolitik an die „traditionellen“ Klientelstaaten im Nahen und Mittleren Osten zurückgekehrt, nachdem man sich das (4) Scheitern des eigenen Disengagement-Ansatzes eingestanden hatte. Demzufolge seien Experimente wie das Reformprojekt in Iran vorwiegend infolge regionaler Zwänge gescheitert. Am explizitesten findet sich diese Einschätzung bei Roby Barrett, der für das Ende des Jahres 1963 eine Verschlechterung der US-Position in Südwest- und Südasien verglichen mit 1960 konstatiert. Er führt diese auf die fehlgeleitete Politik der Kennedy-Administration zurück, die durch ihre politischen Initiativen gegenüber den nichtpaktgebundenen Schwergewichten VAR und Indien große Erwartungen geweckt und diese dann - im wachsenden Bewusstsein der politischen Realitäten - zu enttäuschen gezwungen war: „The planned great opening to the non-aligned world collapsed [...]. “128 Entgegen der ursprünglichen Absicht seien den USA am Ende nur die Allianzen mit den konservativen Regimen in Jordanien und Saudi-Arabien sowie den britischen Klientelstaaten auf der Arabischen Halbinsel geblieben. In eine ähnliche Richtung zielt April Summitt, die die Rückkehr zu ,familiar patterns“ in der amerikanischen Nahostpolitik in der Spätphase der Kennedy-Administration als richtigen Schritt angesichts des Fortbestehens der strategischen Interessen am nahöstlichen Öl und dem Ziel der Eindämmung der Sowjetunion

\footnotetext{
${ }^{127}$ Douglas Letter to Malek Mansur Ġašğà i, 19.7.62, , Ghashghai, Malek Mansour, 1953-1973‘, Box 332, Douglas Papers, LOC; vgl. MemCon, 12.4.62, FRUS 61-63, XVII, S. 599; zu den neuen Stationen, wenig überraschend von Holmes zur Bekräftigung seiner Militärhilfewünsche instrumentalisiert, siehe Tehran \#630, 19.2.62, 788.5/21862, CF 60-63; das State Department warnte allerdings auch vor einer weiteren Ausdehnung der amerikanischen Präsenz in Iran, nicht zuletzt aufgrund der ungeklärten Immunitätsfrage. Vgl. Hilsman Memo, 2.1.62, ,3-A Defense Affairs', NEA, GTI: RIAD, 1958-63, Box 6; Douglas zufolge hatte Allen Dulles ihm früher über die Erpressungsversuche des Schahs berichtet, bei Kürzung der Wirtschaftshilfe z.B. den Grenzübertritt amerikanischer Agenten zu unterbinden. Vgl. Komer Memo, 22.8.62, FRUS 61-63, XVII-XVIII-XX-XXI Suppl., Dok. 96.

${ }^{128}$ BARRETT, Greater Middle East, S. 312; zum Vergleich zu 1960 siehe ebd., S. 317; Barretts hauptsächliche Argumentation ist die Beweisführung zugunsten einer überlegenen Nahoststrategie der EisenhowerAdministration im Vergleich zur nachfolgenden Regierung. Tatsächlich ist der Nahe und Mittlere Osten immer einer der „wunden Punkte“ im Argumentationsgefüge der Eisenhower-Revisionisten gewesen. Vgl. MCMAHON, Eisenhower and Third World Nationalism.
} 
interpretiert. ${ }^{129}$

Diese Interpretationslinie orientiert sich an den frühen Bewertungen der Kennedy'schen Außenpolitik nach Freigabe der ersten Quellen, die - in Umkehrung der „klassischen“ Abfolge historiographischer Einschätzungen der Politik amerikanischer Präsidenten - mit umfassender Kritik an der Regierungsführung der New Frontier einsetzte. Insbesondere die frühen Untersuchungen von Douglas Little prägten die späteren Interpretationen der Nahostpolitik Kennedys als eine Serie von Fehlschlägen. ${ }^{130}$ Allerdings basiert diese Gesamteinschätzung nicht selten auf einem begrenzten Verständnis der eigentlichen Zielsetzung der neuen regionalen Politik Kennedys. Insbesondere das Rapprochement gegenüber dem Ägypten Nassers zielte eben nicht auf die Anbindung der Kräfte des arabischen Nationalismus an die globale westliche Strategie im Rahmen der Eindämmungsdoktrin. Das institutionelle Gedächtnis in Washington erinnerte sich durchaus an die gravierenden Folgen solcher Versuche in den 1950er Jahren und an die verlustreiche Konfrontation mit Nasser aus der Perspektive westlicher Interessen. Stattdessen war die konzeptionelle Zielsetzung begrenzter und entsprach der Abkehr von der interventionistisch umgesetzten Hegemonialstrategie der USA unter Eisenhower nach 1956 hin zu einer - wenn auch in vielem inkonsequenten - Offshore Balancing-Regionalstrategie, einer flexiblen wie anpassungsfähigen Ausgleichspolitik, die im Kern auf die Aufrechterhaltung einer regionalen Machtbalance setzte. Inkonsequent war die neue Strategie insofern, als man keineswegs auf ein für eine solche Gesamtstrategie gleichsam idealtypisches „Disengagement“ setzte, sondern ein Mikromanagement des Gleichgewichts der Mächte im Nahen Osten anstrebte. Limitierte Distanzierung von der regionalen Politik gemeinsam mit einer unvoreingenommenen Haltung gegenüber nationalistischen und neutralistischen Strömungen sowie Reformdruck gerichtet auf die konservativen Allianzpartner sollten gegenseitige Subversion und Instabilität sowie zwischenstaatliche Konflikte, insbesondere ein Wiederaufflammen des arabisch-israelischen Kernkonflikts, verhindern bzw. deren Auswirkungen auf vitale amerikanische Interessen begrenzen. Bob Komer hatte diese Gemengelage bereits im Dezember 1961 in einer weitsichtigen Analyse des angehenden „Nasser-Experiments“ ausformuliert, als er eine „strictly limited marriage of convenience“ als maximales Ergebnis ausgab, da „our interests run sharply athwart each other at too many points.":

But what we might hope to achieve through a more professedly forthcoming policy is to create a vested interest on his part in better US/UAR relations, which would in itself inhibit him from taking actions which would upset it. Let us remember that Nasser could cause us a lot more trouble if he were actively hostile - - in Libya, North Africa, Sudan, Syria, or on Suez transit and ME \{Middle East; R.P.\} oil, to mention but a few instances. Thus perhaps our greatest gains would be negative, we would not get a great deal from Nasser, but at least we might restrain him from doing a lot of things we don't like. Finally, let's not forget we also share certain

\footnotetext{
${ }^{129} \mathrm{Vgl}$. SuMmiTt, JFK and U.S.-Middle East, S. 221f., 241.

130 Vgl. insbesondere LiTTLE, Seeking Order; tatsächlich ist die Argumentation von Little trotz begrenztem Quellenzugang in der Regel ausgewogener als die seiner Nachfolger.
} 
common interests with Nasser -- he's as anxious as we are not to see undue Soviet penetration into the Middle East. A more satisfactory relationship would enable us to talk more frankly with him about where we agree as well as disagree. ${ }^{131}$

Nimmt man diese begrenzte Ausgangsposition als Maßstab, erscheint die Charakterisierung der Kennedy“schen Nahostpolitik durch Douglas Little als Reise von „even-handed“ zu „emptyhanded“ als überzogen. ${ }^{132}$ Im Rahmen ihrer Grenzen kann die begrenzte DisengagementStrategie der Kennedy-Administration, gerade hinsichtlich der Beziehungen zwischen den USA und dem arabischen Nationalismus, durchaus als Erfolg gewertet werden. Arabischisraelische Spannungen blieben in der „Icebox“, wie seitens der Ägypter zugesagt. ${ }^{133}$ Dominierend war dagegen der „Arabische Kalte Krieg“ zwischen radikalen arabischen Nationalismus und dessen Widersachern, mit dem ägyptisch-saudischen Konflikt im Mittelpunkt. ${ }^{134}$ Die flexible amerikanische Politik im Jemen-Konflikt ab 1962 vermied eine einseitige Parteinahme, begrenzte sowohl sowjetische Penetration in Südarabien wie auch eine Rückorientierung Ägyptens nach Moskau, hielt aber auch zugleich Nasser von außenpolitischen Abenteuern gegenüber Saudi-Arabien ab. Insgesamt trug die US-Haltung dazu bei, ein weiteres Ausgreifen des radikalen arabischen Nationalismus in der Nahostregion einzudämmen und den Konflikt in Südarabien zu begrenzen. Gleichzeitig verschloss man sich gegenüber den Wünschen der konservativen Bündnispartner wie Saudi-Arabien und Iran wie auch Großbritanniens, die direkte Konfrontation mit Nasser wiederaufzunehmen, was sich letzten Endes nur negativ auf die US-Interessen ausgewirkt hätte. Das letztendliche Scheitern des Disengagement-Eperiments erfolgte im Grunde erst Ende 1966, als sich innenpolitischer Druck, vor allem pro-israelische Interessen im US-Kongress, durchsetzte und die als proägyptisch diffamierte Ausgleichspolitik beendete - von hier führt eine direkte Kausalkette zum Ausbruch des dritten arabisch-israelischen Kriegs von $1967 .^{135}$

\footnotetext{
${ }^{131}$ Komer Memo, 8.12.61, FRUS 61-63, XVII, S. 359; vgl. auch State Paper, 24.5.62, ebd., S. 677-82.

${ }^{132}$ LITTLE, Seeking Order, S. 177; vgl. dazu die scharf kritischen Einschätzungen von PETERSEN, Decline of AngloAmerican, S. 21; BARRETT, Greater Middle East, S. $316 \mathrm{ff}$; d durchweg mit kritischem Unterton insbesondere bezüglich der Jemen-Politik der ehemalige Botschafter HART, Security Partnership; die fehlenden Erfolge betont SPIEGEL, Other Arab-Israeli Conflict, S. 94-117; positive Einschätzungen der Strategie Kennedys gegenüber Israel in BASS, Support Any Friend, S. 248; verfügbare Quellen demonstrieren allerdings die herausgehobene Bedeutung des innenpolitischen Faktors trotz des - verglichen mit späteren Jahrzehnten - begrenzten Organisationsgrades jüdisch-amerikanischer Lobbyorganisationen. NE-Direktor Robert C. Strong berichtete einem befreundeten Diplomaten im November 1961 die „Israelis have lost none of their drive and are still found in every crack and corner of the USG \{U.S. Government; R.P.\}; Strong Letter, 15.11.61, ,Middle East General, Jan.-Dec. 1961', Records of the Director (NE): 1958-1963, Box 5, RG 59, NACP; letzten Endes kann die innenpolitische Unterminierung der Kennedy'schen Grand Strategy als die Hauptursache für das spätere Scheitern der US-Politik 1966/67 herangeführt werden. Israel, die konservativen arabischen Staaten, die Peripheriemächte Türkei, Iran und Äthiopien wie auch der britische Verbündete versuchten allesamt, das Rapprochement mit Nasser zu unterminieren, was letzten Endes mit der vom Kongress erzwungenen Einstellung der Nahrungsmittelhilfe im Rahmen des PL-480-Programms gelang. Entscheidend für die neuerliche Eskalation des arabisch-israelischen Konfliktes nach der ,ruhigen Dekade“ war aber vor allem das amerikanische Unvermögen, das israelische Nuklearwaffenprogramm zu stoppen. Zu dem die US-Politik stark behindernden Gruening Amendment des USKongresses vgl. SuMMITT, JFK and U.S.-Middle East, S. 216-9.

133 „Icebox“ war die von den Ägyptern gewählte Umschreibung für das „Einfrieren“ des Konflikts mit Israel. Siehe z.B. Brubeck Memo, 21.6.62, FRUS 61-63, XVII, S. $744 \mathrm{ff}$.

${ }^{134}$ Vgl. KerR, Arab Cold War; KAufMAn, Arab Middle East; Gerges, Superpowers and the Middle East.

${ }^{135}$ Zum Ende des „Kennedy-Experiments“ vgl. Wriggins/Saunders Memo, 1.12.66, FRUS 64-68, XVIII, S. 694ff.; Cairo \#3278, 10.12.66, ,IRG/NEA‘, Lot 68 D 117, ASS/NEA: Subject Files 1965-66, Box 5.
} 
Der Autor der vorliegenden Studie vertritt somit die Auffassung, dass die Strategie der Kennedy-Administration gegenüber dem Nahen und Mittleren Osten, ungeachtet der ohnehin nicht vermeidbaren und zwangsläufigen Auf und Abs in der Implementierung solcherlei komplexer Politikentwürfe, letztlich von Kontinuität geprägt war. Was immer die Aufgabe des reformistischen Interventionismus in Iran verursacht hat - diese Kehrtwende war nicht Bestandteil einer grundsätzlichen Neuorientierung in der amerikanischen Nahostpolitik. ${ }^{136}$ Chronologisch lässt sich auch keine Kausalität mit - ohnehin begrenzten - Re-Justierungen im Laufe des Jahres 1963 konstruieren, lässt sich doch die (informelle) Aufgabe des Interventionismus um die Jahreswende 1961-1962 datieren: Sie fällt damit sogar in die Blütezeit des „Nasser-Experiments“ und kann somit unmöglich ein Ergebnis des Scheiterns desselben sein.

Andererseits zeigten sich zu dieser Zeit durchaus erste Tendenzen eines Rollenwandels Irans in der Wahrnehmung durch Washington, die eine Metamorphose des Landes im regionalen Kontext von der einer Sollbruchstelle der (verbliebenen) westlichen Stellung hin zu einem möglichen Pfeiler derselben vorzeichneten. Somit existiert eine zweite „regionale These“, derzufolge die Aufgabe des reformistischen Interventionismus nicht etwa auf das Scheitern der Disengagement-Strategie der Administration, sondern vielmehr auf eine (5) Neubewertung der strategischen Grundzusammenhänge und der zukünftigen Konstellationen in der Region zurückzuführen war, was in der Folge zu einer Neubewertung der Stellung Irans geführt habe. Ausgangspunkt solcher Überlegungen waren jedoch nicht Neueinschätzungen der internen Stabilität Irans. Angesichts der seit 1958 augenfällig gewordenen Begrenzungen westlichen und insbesondere amerikanischen Einflusses im Herzen der arabischen Welt und des gleichzeitigen Rückzugs des traditionellen nahöstlichen Hegemons Großbritannien in die Golfregion und die Peripherie der arabischen Halbinsel - ausgelöst durch den Verlust der letzten zentralen britischen Bastion in der arabischen Welt infolge der irakischen Revolution stellte sich nunmehr die Frage, wie in der Zukunft strategische amerikanische Interessen in der Region gewährleistet werden könnten. Zum Verständnis der Auswirkungen auf die amerikanische Grand Strategy ist eine eingehendere Analyse der wichtigsten strategischen Entwicklungen in der Golfregion und insbesondere der weiteren Transformation des latenten anglo-amerikanischen Kondominiums während der Kennedy-Ära vonnöten.

Auf die in Kapitel III geschilderte vorsichtige Distanzierung von Großbritannien im Nahen und Mittleren Osten und die weitere Aushöhlung des früheren latenten anglo-amerikanischen Kondominiums in der Region im Anschluss an die irakische Revolution folgte eine

\footnotetext{
${ }^{136}$ Ohne Frage war die iranische Führung über die amerikanische Nasser-Politik nicht glücklich. Eine Verbindung zwischen reformistischen Interventionismus in Iran und Rapprochement gegenüber dem Nasserismus lässt sich allerdings nicht feststellen. Vgl. Tehran \#954, 23.5.62, FRUS 61-63, XVII, S. 674f.; Komer kommentierte: „On the other hand the Shah, Saud, Hussein, and the Israelis are all vocally unhappy at the shift in our policy. They are not persuaded by our argument that if we don't help Nasser he'll become even more mortgaged to Moscow."; Komer Memo, 28.5.62, ebd., S. 686.
} 
vermeintliche Erneuerung der special relationship - personifiziert im persönlichen Verhältnis zwischen Kennedy und dem britischen Premier Harold Macmillan -, begleitet von einem Wiederaufleben britischer Hoffnungen auf neuerliche enge Kooperation im Nahen und Mittleren Osten. ${ }^{137}$ Das im Oktober 1957 eingeleitete Experiment einer institutionellen Umsetzung der ,Interdependence“-Doktrin mit der Schaffung anglo-amerikanischer Working Groups, darunter eine zu nahöstlichen Themen, war infolge mangelnden amerikanischen Interesses bald eingeschlafen. Formal hielten die USA an den post-Suez-Vereinbarungen vom Sommer 1957 fest, wonach beide Mächte ,[...] should cooperate to preserve beneficial U.K. relationships with the Persian Gulf and Arabian See principalities of special importance to the supply of oil to the free world, including especially Kuwait, Bahrain, Qatar, Sharja and Muscat."138 Wie bereits geschildert, hatte sich die im Anschluss an die Suez-Affäre noch konsequent verfolgte amerikanische Unterstützung für die Aufrechterhaltung der britischen Stellung als bedeutende nahöstliche Macht bereits vor der irakischen Revolution von $1958 \mathrm{zu}$ einer Unterstützung eines starken Großbritanniens als wichtiges Mitglied der westlichen Allianz gewandelt. Die britische Position in der Region ,an sich“ war im Rahmen der amerikanischen Globalstrategie somit nur noch von untergeordnetem Interesse. ${ }^{139}$ Die amerikanische strategische Umorientierung hin $\mathrm{zu}$ einer - wenn auch vorsichtigen Annäherung an die dominierende regionale Kraft des arabischen Nationalismus verstärkte diesen Effekt einer weiteren Aushöhlung des Kondominiums zusätzlich. Ein britisches Planungspapier aus dem Jahr 1960 zeigte sich zwar insgesamt zufrieden mit der Umsetzung der special relationship in der Nahostregion, kritisierte aber zugleich die grundsätzlich unterschiedlichen strategischen Präferenzen der beiden Mächte. US-Interessen, so das Papier, seien , ,[...] overwhelmingly absorbed in the Communist threat and that the Americans tend to regard everything else as of subordinate importance, whereas two other problems figure largely and assume co-ordinate importance in our thinking: radical nationalism, and the security of our oil supplies which is threatened both by Communist penetration and by radical nationalism. " 140

Trotz dieser Gegensätze - die sich gerade und vor allem in strategischen Differenzen gegenüber dem post-revolutionären Irak unter Qāsim bemerkbar machten ${ }^{141}$ - setzte sich die verbliebene Interessenkongruenz in der Kuwait-Krise von 1961 scheinbar eindrucksvoll durch. Ausgangspunkt der Krise war das Drängen des Emirs von Kuwait auf eine Modernisierung der anachronistischen Vertragsbeziehungen zu Großbritannien. Mit dem Austausch diplomatischer

\footnotetext{
${ }^{137}$ Zum Gesamtzusammenhang mit kenntnisreichen Einschätzungen bezüglich der Nahostregion siehe vor allem die umfassende Re-Interpretation von ASHTON, Golden Days.

${ }^{138}$ Agreed US-UK Paper, encl. to Henderson Memo, 20 Jun. 1957, FRUS 55-57, XII, S. 552; auch angehängt an Memo for Macmillan, 28.6.57, PREM 11/1937; Trevelyan Minute, 28 May 1957, FO 371/, TNA:PRO; vgl. OvENDALE, Transfer of Power, pp. 185ff.; zum Niedergang des Working Groups-Experiments vgl. JONES, Working Group Experiment.

${ }_{139}$ „usefulness per se of the maintenance of a British position“ aus der Staff Study zu NSC 5801 vom Januar 1958. Siehe oben, S. 180 .

${ }_{140}$ Planning Section Report, P.U.S.D., ZP 1/35 G, 5.1.60, CAB 21/5552, TNA:PRO; diese unterschiedlichen Sichtweise machten sich in erster Linie in der Politik gegenüber Nasser und dem neuen Irak bemerkbar.

${ }^{141}$ Vgl. POPP, Working Relationship, S. $411 \mathrm{ff}$; BLACKWELl, Desert Squall; WORRALl, Coping.
} 
Noten am 19. Juni 1961 zwischen dem Emir und dem Political Resident im Persischen Golf, Sir William Luce, wurde Kuwait formal in die Unabhängigkeit entlassen, von vielen Beobachtern als Probelauf für die Zukunft auch der anderen „Dependencies“ am Unteren Golf betrachtet. ${ }^{142}$ Nur eine Woche später protestierte Bagdad gegen diese Entwicklung und bekräftigte die alten Ansprüche auf Kuwait als untrennbaren Bestandteil des Iraks. Die irakischen Drohungen, Kuwait zu „befreien“, und die Zuspitzung der Krise verstärkten die Bedeutung der ebenfalls im kuwaitisch-britischen Notenaustausch enthaltenen fortgesetzten Beistandsgarantie Londons. ${ }^{143}$ Aus Sicht Londons war Kuwait trotz der nun gewährten Unabhängigkeit weiterhin von zentraler strategischer, aber auch wirtschaftlicher und finanzieller Bedeutung. British Petroleum hielt 50\% Anteil an der Kuwait Oil Company, zudem hatte der Herrscher 1960 die potentiell lukrative Offshore-Konzession Shell Oil überlassen für beide Konzerne waren ihre kuwaitischen Positionen von essentieller Bedeutung. Etwa 40\% des Gesamtbedarfs an Öl für die britische Wirtschaft wurde allein aus dem Emirat bezogen zudem akzeptierte die kuwaitische Führung Bezahlung in Sterling und re-investierte einen Großteil der Einnahmen, etwa $£ 300$ Millionen, in die britische Wirtschaft, ein kaum zu ersetzender Beitrag zugunsten der britischen Zahlungsbilanz. ${ }^{144}$ Kaum übertrieben war der Tagebucheintrag Macmillans, demzufolge „Kuwait, with its massive oil production, is the key to the economic life of Britain."145

Mit der Kuwait-Krise von 1961 war für Großbritannien der Ernstfall eingetreten, für den man seit der Irakischen Revolution von 1958 fortwährend Notfallpläne entworfen hatte, wobei sich der amerikanische Partner durchweg ernsthaften gemeinsamen militärischen Planungen entzogen hatte. ${ }^{146}$ Tatsächlich waren amerikanische Zweifel an der Zweckmäßigkeit militärischer Machtmittel zur Sicherstellung von Erdöllieferungen aus dem Persischen Golf einer der Hauptgründe für die Neuformulierung der amerikanischen Nahoststrategie (NSC 6011) vom Juli 1960 gewesen. Assistant Secretary of State Rountree hatte bereits im Juni 1959 generell vor einem Waffeneinsatz und gleichzeitig vor allzu großer Nähe zu London gewarnt

\footnotetext{
${ }^{142}$ Kuwait hatte bereits in den Jahren zuvor den Protektoratsstatus schrittweise abgelöst. Bereits 1958 regulierte Kuwait seine Beziehungen mit anderen arabischen Staaten selbstständig und begann, internationalen Organisationen beizutreten. Mit dem Notenaustausch vom Juni 1961 wurde der Schutzvertrag von 1899 aufgehoben. Zur graduellen Durchsetzung kuwaitischer Handlungsfreiheit gegenüber dem früheren Protektor vgl. SMITH, Neo-Colony.

${ }^{143}$ Vgl. Elwood Memo, 26.6.61, FRUS 61-63, XVII, S. 159-62; vgl. OvENDALE, Transfer of Power, S. $226 \mathrm{ff}$.

144 Vgl. Heath Memo, 2.11.61, C.(61)140, plus Annex Report by Officials, ,Kuwait‘, September 1961, CAB 129/106; die anderen 50\% gehörten der amerikanischen Gulf Oil Company, wobei das Gros des Produktionsanteils der letzteren zu äußerst günstigen Konditionen wiederum von Shell Oil aufgekauft wurde. 1960 bezog British Petroleum 98\% seiner Öllieferungen aus dem Nahen und Mittleren Osten und insgesamt 51\% allein aus Kuwait. Shell bezog 40\% seines Öls außerhalb der westlichen Hemisphäre aus Kuwait. Vgl. Report ,Future Policy in the Perrsian Gulf: Kuwait‘, 25.9.61, CAB 130/178, TNA:PRO.

${ }^{145}$ Zit. aus SMITH, Neo-Colony, S. 161; das Überleben Kuwaits als unabhängiger Produzent - zumal als drittgrößter Produzent überhaupt - war zudem eine Rückversicherung gegen eine möglicherweise drohende Marktkontrolle durch andere nahöstliche Staaten: „Because of its independence, affluence and friendship with us Kuwait stands in the way of a consolidation of control of Middle East oil by one or more of the remaining major Middle East producers (Iraq, Saudi Arabia and Iran) or transit States (the United Arab Republic), and thus provides a valuable insurance that oil will continue to flow from the Middle East in adequate quantities and on reasonable terms [...]."; Report by Officials, ,Kuwait', vorherig. Anm.

${ }_{146}$ Vgl. SMITH, Revival and Fall, S. $118 \mathrm{f}$;; BLACKWELL, Transfer of Power, S. $176 \mathrm{f}$.
} 
(,serious divergencies exist between the British and ourselves on the issues and situations which might justify the use of force in the Persian Gulf area, especially in respect of Kuwait.") und geradezu axiomatisch geschlussfolgert: , ,...] the adverse consequences of the use of force to maintain access to Near Eastern oil on reasonable terms would be such as to make military force impracticable in the long term." ${ }^{147}$ Eine interne Studie 1959 kam zu dem Schluss, es gebe kaum glaubwürdige Szenarien, in welchen der Einsatz militärischer Macht zur Sicherung der westlichen Ölversorgung effektiv und geboten erscheine. ${ }^{148}$

Trotz dieser Überzeugungen blieb der Kennedy-Administration im Kontext der Kuwait-Krise im Sommer 1961 nichts anderes übrig, als Großbritannien zu unterstützen, zumal die irakische Bedrohung zwischenzeitlich real erschien. ${ }^{149}$ Ende Juni 1961 entschied Präsident Kennedy zugunsten „full political and logistic support“ für Großbritannien im Falle einer notwendigen Intervention in Kuwait gegen irakische Versuche einer Übernahme. ${ }^{150}$ Auf britisches Drängen erbat der Emir schließlich militärischen Schutz und 8.000 britische Soldaten nahmen Anfang Juli in bzw. nahe Kuwait Stellung. Amerikanische Unterstützung für die britische Operation Vantage war begrenzt. Kennedy stimmte einem kurzzeitigen symbolischen Richtungswechsel nach Norden einer vor der Küste Ostafrikas operierenden US-Flottille namens Solant Amity zu, offenbar als Unterstützungsgeste gedacht und die USA gaben den Briten zusätzlich politischen Beistand in der UNO. ${ }^{151}$ Ähnlich wie in der Situation nach den Interventionen von 1958 stellte sich allerdings im Anschluss bald die Frage, wie sich ein umgehender Abzug der Truppen politisch angemessen arrangieren lasse. ${ }^{152}$

Die Schwierigkeiten im Anschluss an die Intervention, eine tragbare und dauerhafte Lösung für die Sicherheit Kuwaits zu finden, bestätigte im Kern die bereits seit Anfang 1958 etablierte amerikanische Sichtweise, dass es keine militärischen Lösungen für Probleme im

\footnotetext{
${ }^{147}$ Rountree/Smith Memo, 30.6.59, FRUS 58-60, XII, S. 788; ähnlich und in der politischen Analyse noch umfassender bereits die Behandlung der Frage in einem Memorandum des Planning Board vom Juli 1958: ,If armed force must be used to help retain this area (or even if there is a public indication of willingness to use force), the benefits of any actions in the direction of accommodation with radical Pan-Arab nationalism will be largely lost and U.S. relations with neutral countries elsewhere would be adversely affected. Such accommodation would better provide the basis for continued assurance of access to Kuwait and Persian Gulf oil."; NSC Planning Board Paper, 29.7.58, ebd., S. 119; Hintergründe zu den gemeinsamen anglo-amerikanischen militärischen Besprechungen (und deren Beschränkungen) in Dennison Memo, 29.6.59, ebd., S. 224ff.

${ }^{148}$ Vgl. Study, The Problem of the Use of Military Force to Maintain Access to Near Eastern Oil', 7.4.59, encl. to Reinhardt Memo, 31.3.\{sic $\} 1959$, , Near \& Middle East 1959-61‘, Lot 67 D 548, S/P Records: 1957-1961, Box 154; dies ist vermutlich eine frühere Fassung der Studie, auf die im Rountree/Smith-Memorandum Bezug genommen wurde. Vgl. FRUS 58-60, XII, S. 788 n2; siehe auch Mathews Memo, 21.7.59, ebd., S. 227; die Studie nannte den post-revolutionären Irak als ,[...] prime example of the thesis that a violent political upheaval need not lead to an interruption of the flow of oil or even to an immediate demand for nationalization or for a large increase in oil revenues."; selbst im Falle einer kommunistischen Machtübernahme würde eine irakische Regierung die Öllieferungen an den Westen wohl fortsetzen, so die Schlussfolgerung. Die Studie vom April 1959 demonstrierte im Übrigen wieder die strategische Bedeutung Irans als Erdöllieferant im Falle eines Boykotts.

149 Siehe Home Letter, 28.6.61, FRUS 61-63, XVII, S. 168; ebd., S. 168 n2; vgl. Cabinet Memo by Watkinson, 13.4.62, BDEE A, 4, I, S. 262.

${ }^{150}$ Vgl. ed. note, FRUS 61-63, XVII, S. 172; allerdings entstand rasch der Eindruck, London habe die eigentliche Bedrohung bewusst übertrieben, um eine militärische Demonstration zu legitimieren. Vgl. SMITH, Revival and Fall, S. 120f.

151 Vgl. Home Letter, 2.7.61; ed. notes, FRUS 61-63, XVII, S. 176f., 178, 179; vgl. Talbot Memo, 1.7.61, ,Memoranda to the Secretary and [...]', NEA/ARP: 1958-63, Box 3.

${ }^{152}$ Vgl. State \#165, 12.7.61, FRUS 61-63, XVII, S. 185.
} 
Zusammenhang mit der Ausbreitung des arabischen Nationalismus gab. Washington blieb zwar weiterhin oberflächlich solidarisch mit London, suchte aber bald nach multilateralen bzw. regionalen Lösungen. Angesichts des Dualismus zwischen den verschiedenen Spielarten des arabischen Nationalismus in Kairo und Bagdad bot sich eine Übernahme der Rolle der britischen Truppen durch eine multilaterale Friedenstruppe der Arabischen Liga an. ${ }^{153}$ Die letzten britischen Truppen verließen Kuwait somit im Oktober 1961. ${ }^{154}$ Allerdings brachte die Multilateralisierung der Frage keine dauerhafte Lösung. In Reaktion auf die britische Intervention und die amerikanische Haltung rückte Qāsim den Irak wieder näher an das sozialistische Lager, beendete die zuvor praktizierte Unterdrückung der Kommunistischen Partei und bekräftigte im Dezember 1961 erneut die Ansprüche auf Kuwait. ${ }^{155}$ Der Nahostveteran John Jernegan, inzwischen Botschafter in Irak, warnte vor den langfristigen Folgen der US-Haltung während der Kuwait-Krise. Qāsim sei offenbar entschlossen, den Druck aufrechtzuerhalten und spiele mit dem Gedanken die britische Sicherheitsgarantie für Kuwait zu zermürben, indem er weitere Interventionen der britischen Schutzmacht durch entsprechende militärische Vorbereitungen erzwinge. Eine solche Strategie würde sowohl die multilaterale Garantie der Arabischen Liga als Feigenblatt enttarnen, die „Schein-Unabhängigkeit“ Kuwaits entlarven und die neo-imperialistischen Ambitionen der anglo-amerikanischen Mächte bloßstellen. Die einzige Möglichkeit sei eine wirkliche Unabhängigkeit Kuwaits, eine ReInvestition der exorbitanten kuwaitischen Öleinnahmen in den ärmeren arabischen Staaten (,[...] nothing less than major and continuing fin \{financial; R.P.\} contribution to develop other Arab states [...] will in long run save Kuwait from total absorption by one of its neighbors") und eine Aufgabe der fehlgeleiteten Idee, Großbritannien könne mit seiner militärischen Macht die Lage dauerhaft stabilisieren: „The West can no longer afford \{the; R.P $\}$ present policy of reliance on British military protection, which seems to be $\{$ the $\}$ most attractive one to greedy, short-sighted Shaikhs. “156

Jernegans Argumente veränderten die amerikanische Position bezüglich Kuwaits, doch gingen die letztendlichen Anpassungen der US-Politik nicht so weit wie vom Bagdader Botschafter gewünscht. Zwar machte man seinen Vorschlag, die Kuwaiter zur großzügigen Verteilung der Öleinnahmen zu ermuntern, zur eigenen Politik, verließ sich aber für die Übergangszeit bis zur Akzeptanz der Eigenstaatlichkeit des Emirats weiterhin auf die britische Sicherheitsgarantie, vor allem da es ,would be undesirable to have oil resources of Kuwait and Iraq or Kuwait and

\footnotetext{
${ }_{153}^{159}$ Vgl. Strong Memo, 24.7.61, ebd., S. 197ff.

${ }^{154}$ Eine Arab League Security Force war vom September 1961 bis Februar 1963 an der kuwaitisch-irakischen Grenze stationiert.

${ }^{155}$ Explizit in Talbot Memo, 18.12.61, FRUS 61-63, XVII, S. 364ff.; Qāsim hatte bereits mit der Enteignung fast des gesamten Konzessionsgebiets der Iraq Petroleum Company und deren Tochtergesellschaften außerhalb der aktiven Fördergebiete (0,5\% der ursprünglichen Konzession) im Dezember 1961 reagiert. Vgl. ebd., S. 365 n3.

${ }^{156}$ Baghdad \#286, 28.12.61, FRUS 61-63, XVII, S. 375; Jernegan warnte vor der Reaktion der neuen Länder auf eine mögliche militärische Auseinandersetzung in Kuwait: ,Thought of British troops fighting Arabs on Arab soil with moral and political (if not military) support of US particularly disturbs me as I am sure Afro-Asian world will fail to appreciate distinction which we so clearly see between Kuwait and Goa."; ebd., S. 374.
} 
Saudi Arabia under sway of any single government [...]. “157 Allerdings hatte die Diskussion über Kuwait die Sensibilität über die Frage der zukünftigen Gestaltung der eigenen Golfpolitik und die bestmöglichen Maßnahmen zur Aufrechterhaltung der Stabilität in der Region zweifellos gestärkt. ${ }^{158}$

Im Februar 1962 ging Walt Rostows Policy Planning Council auf Anfrage von NEA eine umfassende Studie zum nahöstlichen Teil des eurasischen „Rimland“ an, aus der unter anderem erste Studien zur Funktion des Persischen Golfs im internationalen Staatensystem im Allgemeinen und im Kontext des Kalten Krieges im Besonderen resultierten. ${ }^{159}$ Autor war der Nahostexperte William Polk im Policy Planning Council, in der Iranpolitik ein Sympathisant der Komer-Hansen-Gruppe, und Verfasser einer Reihe von provokanten wie intern geschätzten Studien zu regionalen Problemstellungen. Polks erster Entwurf, eine 80-seitige Analyse mit dem Titel „The Persian Gulf in Middle Eastern Politics“ vom Sommer 1962, war ein Versuch, die strukturellen Konfliktkonstellationen einer langfristigen Lösung zuzuführen. Hauptaugenmerk legte Polk auf die von ihm so genannte Anomalie der „Fehlverteilung“ (,maldistribution“) von Bevölkerung, Bodenschätzen und Kapital innerhalb des Sub-Systems des Nahen und Mittleren Ostens. Das Gros der nahöstlichen Bevölkerung balle sich entlang der Mittelmeerküste, während sich das Investitions-Kapital generierende Erdöl zuvorderst in den ariden und spärlich bevölkerten Gebieten rund um den Persischen Golf fände. Abseits vom arabisch-israelischen Gegensatz sei diese Asymmetrie strukturell verantwortlich für die Konfliktträchtigkeit der Region und den Versuch der größeren Mächte, die regionale Vorherrschaft zu erringen. Die eigenständige Existenz der Golfstaaten sei bislang durch externe Mächte - in der Regel mittels „Kanonenboot-Politik“ sichergestellt worden, doch sei innerhalb der nächsten fünf Jahre mit einer Erosion dieser Taktik angesichts international fortschreitender Dekolonisation und der Ausbreitung des arabischen Nationalismus zu rechnen: „Until and unless the Persian Gulf area is able to win for itself a viable position in the Middle Eastern political structure, there can be little more stability than that of a potential and impending crisis. “160 Polk empfahl eine langfristige Umorientierung der Kapitalinvestitionen

\footnotetext{
${ }^{157}$ Circular Airgram \#CA-951, 26.1.62, FRUS 61-63, XVII, S. 439; die Besorgnis über die neue Politik führte auch zu einer Infragestellung der häufig als wait and see-Politik charakterisierten eigenen NichtInterventionspolitik gegenüber Irak. Vgl. Komer Memo, 4.6.62; Brubeck Memo, 20.6.62, ebd., S. 705, 740-4; vgl. State Policy Directive PD/NEA 3.1, , Preservation of Kuwait's Integrity“, 15.3.62, ,Department of State, Policy Directives, NEA [... $]^{\star}$, NSF, D\&A, Box 288, JFKL.

${ }^{158}$ Eine solche Reaktion entsprach auch dem Modus Operandi der Kennedy-Administration. Ein Planungspapier umriss diese Denkweise mit Bezug auf den Nahen und Mittleren Osten: „There was general agreement that we are too preoccupied with 'reacting' and with maintaining or restoring the status quo. The point was made that we are living in an age of revolution and upheaval when the longer term interests of the United States may not necessarily be advanced by the maintenance or restoration of the status quo."; Cortada Memo, 15.12.61, ,Middle East General, Jan-Dec 1961', Records of the Director (NE): 1958-1963, Box 5, RG 59, NACP \{Hervorheb. i. Orig.\}.

${ }^{159}$ Vgl. Rostow Memos, 18.1./28.2.62; Talbot Memo, 5.2.62, , Near \& Middle East 1962‘, Lot 69 D 121, S/P Records: 1962, Box 222; der Policy Planning Council hatte offenbar bereits auf eigene Initiative erste Vorstudien zur Golfregion anstellt.

${ }^{160}$ Report ,The Persian Gulf in Middle Eastern Politics', o.D., encl. to Polk Memo to Members of the Persian Gulf Oil Planning Group, 5.6.62, ebd.; für die Zeit nach dem Schwinden britischer Macht im Golf drohe ein Konflikt zwischen den Prätendenten VAR, Irak und Saudi-Arabien (aber auch Irans) um die Vorherrschaft im Golf: ,That Arab Leader who succeeded in doing this would destroy in his favor the present internal balance of power in the area and would be in a strong position to subject the entire Arab Middle East to his control.“
} 
der Golfstaaten, generiert durch die Erlöse aus der Ölförderung (1960 für alle inklusive Iran: $\$ 1,423$ Milliarden), weg vom Westen und zugunsten der Entwicklungsprogramme der bevölkerungsreichen arabischen Staaten. ${ }^{161}$ In einem späteren Entwurf vom September 1962, dieses Mal unter dem Titel „Oil and Interdependence in the Middle East“ als Teil von Walt Rostows BNSP, erweiterte Polk seine Analyse um sicherheitspolitische Aspekte und insbesondere um detaillierte Vorhersagen bezüglich der britischen Position im Golf. Der Status Quo im Persischen Golf sei langfristig nicht zu erhalten, was einen größeren regionalen Konflikt möglich erscheinen ließ: „Several of the rim states have conflicting territorial ambitions in the Gulf and are waiting to pounce upon the weaker Shaikhdoms in the event of a British disengagement.“ Mit der beginnenden Erosion der britischen Position sei nach etwa 5 Jahren zu rechnen, womit sich Polk durchaus vorausschauend zeigte. Somit war es im Interesse der USA, sowohl eine stärkere politische wie wirtschaftliche Interdependenz zwischen den Golfstaaten und der weiteren arabischen Welt zu fördern und auf die Lösung der verschiedenen territorialen Konflikte noch vor Verschwinden der britischen Macht hinzuarbeiten:

While there is probably little the US can or need do to change the course of events in the next 5 years, foresighted action on our part during this period may help shape the course of subsequent years, preserve stability in the area and prevent Soviet entry into the Persian Gulf proper. By 1970 the British process of gradual disengagement from the Gulf will probably have reached its final stages; Iran and Iraq may be imminently threatened by Soviet domination; and Saudi Arabia immersed in national turmoil. ${ }^{162}$

Zur Vorbereitung auf den britischen Abzug empfahl Polk übergangsweise die fortgesetzte Unterstützung der britischen Position parallel zu verstärkter amerikanischer Einflussnahme zugunsten der Etablierung einer tragfähigen Folgeordnung, insbesondere der Förderung der Interdependenzen mit der übrigen Region sowie die Vermittlung in Territorialkonflikten. ${ }^{163}$ Für Iran empfahl Polk trotz seines Szenarios für 1970 im Grunde die Umsetzung der oben geschilderten Guidelines-Politik: „Continue to buttress the non-Communist regime in Iran by economic, military and other appropriate assistance, while simultaneously fostering political and social reform. “164 Die innere Entwicklung Irans (und anderer regionaler Staaten) war nicht

\footnotetext{
${ }^{161}$ Ebd; Polks Handlungsempfehlungen fehlen im ersten Entwurf, finden sich aber in einer späteren gekürzten Fassung: „1) promote the integration of the Persian Gulf into the Middle Eastern state system in an orderly fashion and in a way phased with British plans over the coming five year period; 2) accelerate the provision of Middle Eastern capital for constructive development projects in the capital deficient areas to provide an alternative to U.S. and/or Soviet aid";Vgl. Report ,The Persian Gulf in Middle Eastern Politics", 25.6.62, encl. to Polk Memo to Members of Persian Gulf Oil Planning Group, 25.6.62, ebd.

${ }_{162}$ Report ,BNSP Planning Task Number III-H: Oil and Interdependence in the Middle East', o.D., encl. to Rostow Memo, 14.9.62, ebd.; die Policy Recommendations entsprachen weitgehend denen der vorherigen Versionen, mit einer nennenswerten Neuerung: „We review the possibility of the development of an alternative defense capacity for the Gulf, independent of Aden and Bahrain."

${ }^{163}$ In der Folge beschäftigte sich das State Department zum ersten Mals seit Längerem systematisch mit den vielfältigen Territorial- und Grenzkonflikten in der Region des Persischen Golfs. Vgl. Rostow Memo, 17.1.62; Chayes Memo, 4.5.62, ebd.

${ }_{164}$ Report ,Oil and Interdependence‘, ebd.; das Doomsday-Szenario im Jahr 1970 ist wieder repräsentativ für die allgemein pessimistischen langfristigen Erwartungen für Iran. Für Irak empfahl die Studie, den zu erwartenden Sturz Qāsims abzuwarten im Sinne der wait and see-Politik und anschließend ein arabisch-nationalistisches Nachfolgeregime durch direkte Unterstützung wieder pro-westlich auszurichten. Für Saudi-Arabien empfahl die Studie fortgesetzten Rapport mit ,educated, nationalist and young military elements in Saudi Arabia in preparation for the day when one or a combination of these groups comes into power", die damalige allgemeine
} 
eigentlicher Gegenstand von Polks Studie, doch illustriert seine Haltung - gerade als überzeugter reformistischer Interventionist - den inzwischen etablierten Konsens, dass bei aller Reformnotwendigkeit in Iran eine Ablösung der unzweifelhaft anti-kommunistischen Führung des Schahs angesichts der zentralen strategischen Bedeutung des Landes nicht riskiert werden durfte. ${ }^{165}$

Die Überlegungen für eine neue konzeptionelle Ausrichtung der amerikanischen Golfpolitik, wie sie im Policy Planning Council über das Jahr 1962 diskutiert wurde, bestätigten im Kern die auch zuvor praktizierte US-Politik einer vorwiegend deklaratorischen Unterstützung der britischen Position bei gleichzeitigem Vermeiden einer öffentlichen Identifikation mit derselben - im Grundsatz die Fortsetzung einer der zentralen Leitlinien amerikanischer Politik im Kontext des latenten anglo-amerikanischen Kondominiums im Nahen und Mittleren Osten seit dem Zweiten Weltkrieg. ${ }^{166}$ Ernsthafte Überlegungen, den eigenen militärischen „Footprint“ in der Region bzw. im Indischen Ozean zu verstärken, gab es zu diesem Zeitpunkt noch nicht, zumal man im Sommer 1962 sogar ein „stiffening of the British resolve concerning this area" wahrzunehmen glaubte. ${ }^{167}$ Auch zeigte man im Laufe des Jahre 1962 kein Interesse an britischen Vorschlägen, anglo-amerikanische Konsultationen über den Persischen Golf abzuhalten. Tatsächlich führte das Übersenden von Polks Studie über „Oil and Interdependence in the Middle East" nach London für erhebliche Verstimmung im angloamerikanischen Verhältnis. Die Briten reagierten äußerst empfindlich auf die Vorhersage des Policy Planning Council, dass mittelfristig mit einem Abzug der britischen Macht aus dem Golf zu rechnen sei. In Kombination mit der sich abzeichnenden Veränderung der amerikanischen

Erwartungshaltung widerspiegelnd, auch die Monarchien am Golf würden sich nicht langfristig gegen den allgemeinen Trend in der arabischen Welt halten können. Vgl. Brubeck Memo, 22.8.62, ,1962 Memoranda to the White House', Records of the Director (NE): 1958-1963, Box 6, RG 59, NACP.

${ }^{165}$ Polk erwähnte in der Studie auch die Konsequenzen einer Kontrolle Irans im Falle eines allgemeinen Krieges: "In the event of general war conducted on a conventional basis, the Western position in the Gulf would be untenable if the Soviets were to occupy Iran, which would give them direct land access to the Gulf and its principal oil-producing centers."

${ }^{166}$ Ein britischer Offizieller bewertete die amerikanische Haltung zutreffend: „I cannot see that any western power would want to take on tasks that from their point of view are now well performed for them by the British without embarrassment for themselves (this feeling is no doubt behind the American reluctance to make joint military plans with us).“; zit. aus SMITH, Revival and Fall, S. 124; FAIN, Managing, S. 105ff.; Blackwell ist in seiner Wertung zuzustimmen, dass die Konstellation nach der Kuwait-Krise amerikanische Dominanz innerhalb der special relationship dokumentierte: ,[...] the Kennedy Administration manipulated the alliance to serve the American national interest in a way that was far more successful than Macmillan's attempts to achieve the reverse outcome in 1957-58."; BLACKWELL, Transfer of Power, S. 178

167 Newsom Letter, 14.5.62, ,Jidda 1962', Records of the Director (NE): 1958-1963, Box 6; nach einigen unbedachten Äußerungen des britischen Verteidigungsministers in Gesprächen mit McNamara hatte letzterer eine gemeinsame State Department-DOD-Studie für den Fall eines britischen Rückzugs aus Asien angeregt. Die Lage hatte sich allerdings rasch wieder beruhigt. Vgl. Rusk Letter, 5.10.61; Johnson Memo, 3.10.61; Blitgen Memo, 30.11.61, ,Misc (1959-63) folder no. 1', Entry A1 3063, Office of the Deputy Under Secretary of State for Political Affairs, MDAP Memos, Box 2, RG 59, NACP; zu entsprechenden britischen Überlegungen siehe z.B. Cabinet Committee on Defence Meeting Minutes, 21.5.63; Bishop Minute, 8.6.59, BDEE A, 4, I, S. 267ff.; allerdings begannen nun erste Planungen für eine denkbare zukünftige US-Rolle. Noch ein Jahr früher hatte es starken Widerstand gegen jede Idee der Etablierung einer Indian Ocean Fleet gegeben. Nachdem sich Admiral Burke in einem Zeitungsinterview für die Schaffung einer solchen Flotte ausgesprochen hatte, entgegnete man im Policy Plannig Staff, dass der Vorschlag ,[...] run counter to anticipated developments in the world strategic picture over the next few years, notably a decrease in our reliance on overseas bases and facilities."; der Indische Ozean aus US-Sicht sei ,[...] a matter more of the defense of its entrances and exits than of its entire surface."; Wilson Memo, 29.9.60, ,Near and Middle East 1959-61', Lot 67 D 548, S/P Records: 1957-1961, Box 154; siehe auch Kapitel VI.3. unten. 
Kuwait-Politik wuchs in London der Verdacht, die USA ,[...] may not fully appreciate $\{$ the; R.P.\} extent of both HMG's \{Her Majesty's Government's $\}$ willingness and capability $\{$ to $\}$ preserve its Persian Gulf position and may even be prepared \{to $\}$ write off British interest in full in foreseeable future“, so eine Einschätzung der US-Botschaft. ${ }^{168}$ Sir William Luce, der britische Political Resident im Golf, kritisierte die Polk-Studie als Ausdruck einer angedachten „rather wishy-washy American policy in place of our special position“. Luce argumentierte stattdessen für ein Fortdauern der britischen Präsenz ,auf unbestimmte Zeit“ und eine gemeinsame anglo-amerikanische Anstrengung, die arabische Halbinsel (und Jordanien) zu einer ,bastion against the expansion of Nasserism“ aufzubauen. ${ }^{169}$ Tatsächlich fand diese Idee von Luce durchaus Unterstützung im Foreign Office. Ein britischer Offizieller betonte dabei die Notwendigkeit der Unterstützung durch Washington: „By all means let us educate the Americans and carry them with us in our policy for the Gulf and the Middle East. We shall not get anywhere, if we do not, because the major influence in this area has long ago passed definitively from our hands into those of the Americans." ${ }^{170}$ Insgesamt aber überwog die Skepsis angesichts der weiteren Ausbreitung der arabisch-nationalistischen Ideen und der Zurückhaltung der USA, sich allzu eng mit der britischen Position zu identifizieren. ${ }^{171} \mathrm{Im}$ Grundsatz wurde weiter an der Kuwait-zentrierten britischen Position festgehalten, wenngleich man das Argument der Polk-Studie, letztlich würden kommerzielle und wirtschaftliche Interessen der Ölförderstaaten unabhängig von deren innerer Verfasstheit die zukünftige Versorgung sicherstellen, durchaus auch in London anerkannte. ${ }^{172}$

${ }^{168}$ London \#3922, 8.4.63, POL 7 UK-US, CF 63; dieser Verdacht sei in der Tat durch den Polk-Report verstärkt worden. Aufgrund von Bill Polks Gesprächen mit der kuwaitischen Führung befürchtete man zudem, Washington ,[...] may have in mind encouraging, or perhaps even pressing, Kuwait to allocate an ever increasing share of its annual revenues to econ $\{$ economic $\}$ development of other Arab states, thereby not only loosening ties with UK but also ultimately reducing Kuwait's reserves in UK."; ebd.; vgl. auch Strong Letter, 13.3.63, ,Political Affairs \& relations, Iraq-Kuwait", NEA/ARP: 1958-63, Box 5; gegenüber Kermit Roosevelt beschwerte sich ein britischer Offizieller über den „,spate of uncoordinated American advice“ an „,relatively unsophisticated Kuwaiti minds“, der zu einer Abnahme kuwaitischer Investitionen in London führen könne. Vgl. London \#3940, 9.4.63, POL 7 UKUS, CF 63.

${ }_{169}$ Luce Letter, 17.1.63, FO 371/168632, TNA:PRO; Luce erteile alternativen Ideen zur dauerhaften Sicherung des Zugangs zu den Ölreserven des Golfs eine Absage: ,What we need is firm recognition, by ourselves and other Western powers, particularly America, of the need to maintain our position indefinitely [...] and a robust policy to enable us to do so."; Luce zufolge war es im ureigenen amerikanischen Interesse, die britische Position vorbehaltlos zu unterstützen. Die ,revolutionary disease“ sei nicht „incurable“. Im Falle einer nasseristischen Machtübernahme in Saudi-Arabien empfahl Luce eine anglo-amerikanische Politik zu ,[...] encourage what I believe would be the natural tendency of the country to split up, and take steps to ensure the integrity and stability of the eastern Province (and, if possible, the Najd) with its important sector of Gulf oil production."

${ }^{170}$ Beith Minute, 28.1.63, ebd.

171 Vgl. Hiller Minute, 30.1.63, ebd.; ein anderer Offizieller verteidigte sogar intern die Polk-Studie: „They may even be overestimating our determination to stay in certain circumstances of domestic British politics."; Given Minute, 30.1.63, ebd.; ,I do not think that there is any prospect of getting the present U.S. administration to subscribe to the theory that we must work together to exclude Nasserism from the Arabian Peninsula. Their answer would be that Nasserism is another name for the upsurge of Arab Nationalism and radicalism and that you can no more exclude it from evolving Arab communities than you can withstand a tidal wave."; Stevens Draft Letter, 8.2.63, ebd.

${ }_{172}$,If supply considerations were the only ones, there would be quite a strong case for relying on the fact that the producing countries cannot afford to cut off the flow of oil to the Western markets. After all, the Communist bloc are also driven to try to export their oil to the West. We do not, however, at present rely on this argument because commercial/financial considerations enter and the preservation of Kuwait's independence has been agreed to be a major U.K. interest. Kuwait's independence is in the present circumstances necessary if we are to preserve as favourable as possible a tactical position for the Western oil companies in their dealings with the Middle East governments and as great as possible a profitability in their operations."; Crawford Minute, 4.2.63, ebd.; zur LuceDebatte vgl. SMITH, Revival and Fall, S. 126ff.; zwar bildete die Kuwait-Intervention von 1961 im Kern die Referenz für den politisch-militärischen Posture Großbritanniens im Golf für die 1960er Jahre, doch gab es auch 
Diese innerbritische Debatte verstärkte in London die gefühlte Notwendigkeit, die eigene Position stärker mit der amerikanischen abzustimmen. ${ }^{173}$ Zusätzlich verstärkt wurde dieser Impuls durch die regionalen Entwicklungen im Herbst 1962. Ausgelöst durch den Sturz des zaiditischen Imamats im nördlichen Jemen durch einen Militärcoup im September 1962 und der anschließenden Intervention ägyptischer Truppen zugunsten der von royalistischen Rebellen gefährdeten Jemenitischen Arabischen Republik (JAR), verlagerte sich der intraregionale „Arab Cold War“ nun auf die arabische Halbinsel - die Royalisten wurden bald von Saudi-Arabien, Großbritannien, Jordanien, Iran und sogar Israel unterstützt. ${ }^{174}$ Die KennedyAdministration - im Einklang mit der begrenzten Disengagement-Strategie - favorisierte einen Entflechtungsplan, basierend auf einem gleichzeitigen graduellen Rückzug der ägyptischen Truppen im Gegenzug für die Beendigung der Unterstützung der Rebellen durch die anderen Staaten, mit dem Ziel eine weitere Eskalation des Konflikts um die Führungsrolle in der arabischen Welt zu verhindern. Obwohl diese Anstrengungen ohne Erfolg blieben, anerkannte Washington die neue arabische Republik im Nordjemen am 19. Dezember 1962, gegen den erklärten Willen Londons. ${ }^{175}$ Die noch während der Kuwait-Krise geschlossen erscheinende anglo-amerikanische Front im Nahen und Mittleren Osten war nun auch öffentlich auseinandergebrochen, eine aus britischer Sicht fraglos unerwünschte Entwicklung. Andererseits verstärkte Washington zugleich - und konsequent im Kontext der eigenen Offshore-Balancing-Position - die nun auch öffentliche Unterstützung für die britische Position in Aden. Der Sturz einer konservativen Monarchie auf der arabischen Halbinsel durch pronasseristische Offiziere war dennoch ein negatives Omen für die Zukunft der in erste Linie auf reaktionäre und feudale Scheichs gestützten britischen Position, nun vor allem konzentriert auf die Südarabische Föderation, der im Januar 1963 auch die Kronkolonie Aden beigetreten war. $^{176}$

Angesichts der Auswirkungen des Jemenkonflikts waren nun auch die USA zur

in London eine fortgesetzte Diskussion, inwieweit die Aufrechterhaltung des allein auf die Verteidigung Kuwaits ausgerichteten Verteidigungskomplexes Großbritannien-Ostafrika-Südarabien-Oman-,,Trucial States“-Bahrain wirklich sinnvoll war.

${ }^{173}$ Vgl. Killick Memo, 13.2.63, FO 371/168632, TNA:PRO.

174 Vgl. FAIN, Unfortunate Arabia; GERGES, Superpowers and the Middle East, S. 145-73; BASS, Support Any Friend, S. 98-143.

${ }^{175}$ Vgl. FAIN, Unfortunate Arabia, S. 142f.; zur US-Strategie vgl. Talbot Memo, 13.11.62, FRUS 61-63, XVIII, S. 220ff.; der First Secretary in der US-Botschaft in London, Hermann Eilts, eine zentrale Figur für die USNahostpolitik in den 1970er Jahren, kritisierte im Nachhinein den britischen Verbündeten: „In matters affecting Yemen, the British are incredibly myopic.“; Eilts Letter, 5.2.63, ,Middle East - Personnel 1963', Lot 66 D 5, NEA/NR: Subject Files, 1958-66, Box 3.

176 Die übrigen Protektoratsstaaten außerhalb der Föderation wurden im Protektorat von Südarabien zusammengefasst, das letztlich wie die Föderation im November 1967 in den unabhängigen Südjemen aufging. Die Insurgenz im Südjemen gegen die britische Kolonialherrschaft begann im Dezember 1963. Zu den Folgen vgl. BAlfour-PAul, End of Empire, S. 80ff.; SMITH, Revolution and Reaction, S. 196ff., 202-5; die britische Regierung sah die mittelfristige Gefährdung des Stützpunkts in Aden für etwa 1967 voraus, ging aber nun von der Möglichkeit aus, auch ohne Aden von Singapur aus die Verteidigungszusage für Kuwait einhalten zu können. Vgl. Walmsley Memo, 7.12.62, FO 371/168632; C.C. (62) 59, 9.10.62, CAB 128/36, TNA:PRO. 
Wiederaufnahme der Nahostkonsultationen bereit. ${ }^{177}$ Assistant Secretary of State Phillips Talbot traf sich für einen zweitägigen Gedankenaustausch über die gemeinsame Golfpolitik mit seinen britischen Kollegen in London im April 1963. Hier bestätigten beide Seiten eine ähnliche, in Teilen sogar identische Sichtweise über die zentralen Fragen in der Golfregion. Genauso klar wurde allerdings die Unvereinbarkeit der jeweiligen Positionen gegenüber der Frage, wie man in Zukunft mit der stärksten ideologischen Kraft, dem arabischen Nationalismus nasseristischer Prägung, umzugehen gedachte. Talbot betonte die zentrale Einsicht hinter der amerikanischen Rapprochement-Politik, derzufolge, zumindest zu einem gewissen Teil, „the stabilizing role of the West rests on our relations with the various indigenous forces of the area". Ein „Arab deterrent“" sei die effizienteste Abwehr gegen sowjetische Penetration. Somit war man amerikanischerseits nicht bereit, gemeinsam mit London eine gegen Nasser gerichtete regionale Politik zu verfolgen. Im Gegensatz zur britischen Position, die darauf baute, mit militärischer Präsenz den Status Quo und damit den Zugang zu den Ölreserven zu erhalten, ermahnte Talbot seine Kollegen, dass aus Sicht Washingtons , ,[...] the best way to save access to oil in the Middle East is to avoid adamantly resisting new trends in the area. "178 Die britische Seite betonte dagegen die Abgelegenheit der Golfregion, die noch sehr begrenzte Strahlkraft des arabischen Nationalismus dort, sowie die weiterhin herausragende Bedeutung des ,golden nugget of Kuwait " für die britischen (und westlichen) Ölinteressen. ${ }^{179}$ Im Kern fanden die Positionen also nicht zusammen. Obgleich die Kennedy-Administration an der Zweckmäßigkeit des britischen Ansatzes zweifelte und ein koordiniertes Vorgehen verweigerte, war sie sich dennoch der vitalen Bedeutung der Golfposition für die britische Wirtschaft bewusst und unterstützte daher deklaratorisch weiterhin die Aufrechterhaltung dieser Position, zumal sie vorerst noch vereinbar erschien mit der generellen Entwicklung in der Nahostregion. ${ }^{180}$

Was bedeutete somit die weitere Erosion des latenten anglo-amerikanischen Kondominiums und die nunmehr fast nur noch deklaratorische amerikanische Unterstützung für die britische Position in der Golfregion und auf der Arabischen Halbinsel für die Wahrnehmung Irans durch die Planer in Washington? Tatsächlich stieg das Bewusstsein über die mittelfristige Bedeutung

\footnotetext{
${ }^{177}$ Vgl. Davies Memo, 31.3.64, , [...] 2-b. Persian Gulf Petroleum 1964‘, Lot 67 D 120, NEA/ARP: 1964-66, Box 1.

${ }_{178}$ Zitate aus den Gesprächsprotokollen in London \#A-to State, 31.5.63, POL UK-US, CF 63; die äquivalente britische Version in Records of Meetings, B1052/32G, 23./24.4.63, FO 371/168634; die Briten gaben in den Gesprächen drei hauptsächliche Begründungen für ihre fortgesetzte Präsenz in der Golfregion: (1) Eindämmung kommunistischer Penetration, (2) Sicherstellung der Ölversorgung und (3) Beitrag zur generellen Stabilität und Entwicklung der Region. Allerdings verstanden die Amerikaner im Laufe der Gespräche, dass ,the continued availability of cheap sterling oil" das eigentliche zentrale Anliegen war. Zu den Gesprächen vgl. FAIN, Managing, S. $112 \mathrm{ff}$; SMITH, Revival and Fall, S. 127f.; VON BISMARCK, British Policy in the Persian Gulf, S. 79-84.

${ }_{179}$ Arabian Department Report, 22.4.63, T 312/550; die Rolle Kuwaits als pro-westlicher Swing Producer wirkte sich aus britischer Sicht auch positiv auf die Verhandlungsposition der Companies gegenüber Regierungen in anderen Förderländern aus. Zur herausragenden Bedeutung Kuwaits in der Aufrechterhaltung des „Leitwährungsstatus“ des Pfund Sterling vgl. die detaillierte Untersuchung bei GALPERN, Sterling, S. 198-267.

${ }^{180}$ Somit bestätigte sich hier ein weiteres Mal die Grundausrichtung der amerikanischen Position im Hinblick auf die britische Nahoststellung, die man im Sinne von NSC 5801 von Anfang 1958 unterstützte mit der Intention, die Stellung Großbritanniens als starkes Mitglied der westlichen Allianz an sich zu stützen, also nicht mehr zuvorderst im Kontext der eigentlichen Nahostpolitik.
} 
Irans als bevölkerungsreichster Anrainer des Golfs im Kontext des zunehmenden britischen Machtverlusts und des für die Zukunft absehbaren Rückzugs, selbst wenn der genaue Zeitpunkt eines solchen noch nicht vorauszusehen war. Dass Iran in Zukunft eine größere Rolle spielen würde, war offenkundig. Fraglich ist allerdings, ob diese Aussicht die amerikanische Politik im Kontext des reformistischen Interventionismus beeinflusste.

Wenig überraschen können, angesichts der Selbsteinschätzung sowohl seiner eigenen Bedeutung als Herrscher wie auch seiner Sichtweise auf die historisch legitimierte Führungsrolle seines Landes in der Region, die unablässigen Versuche Mohammad Rezās, Iran für eine Rolle als Stellvertreter westlicher Interessen ins Spiel zu bringen, selbstverständlich mit dem Hintergedanken der in diesem Fall unbestreitbar notwendig werdenden materiellen Transfers zur Stärkung einer derartigen Position, gerade und vor allem was militärische Güter anginge. Die seitens des Schahs vorgebrachte Bedrohung der Nahostregion durch die schleichende "Satellitisierung“ Iraks und Afghanistans war bereits ein etablierter Topos. Während seines Washington-Besuchs im April 1962 hatte Mohammad Rezā die Vorzüge eines unzweideutig pro-westlichen Irans, eingebettet in den Rahmen eines ,positiven Nationalismus“, für die regionale Gesamtlage deutlich herausgehoben. Abgesehen von den arabischen Monarchien hätten alle anderen arabischen Staaten ihre Armeen auf sowjetische Ausrüstung umgestellt und sich Moskau in der Folge auch politisch angenähert. Zugleich gab sich der iranische Monarch besorgt über den weiteren britischen Rückzug aus den Kerngebieten des Nahen und Mittleren Ostens und die Tendenz Londons, sich auf die Großregion südlich von Suez zu konzentrieren, also die Position in Aden und Südarabien und parallel dazu die Basen entlang der Küste Ostafrikas. ${ }^{181}$ Für den Schah drohte mit dem perzipierten britischen Rückzug auf Raten aus dem Golf ein Machtvakuum, das sich Nasser und die VAR zu füllen anschickten, selbst wenn zum damaligen Zeitpunkt nur eine sehr begrenzte Wirkung des radikalen arabischen Nationalismus in den Scheichtümern entlang der Golfküste zu spüren war. Für Mohammad Rezā war Iran der Schlüsselstaat für die Stabilität des Persischen Golfs und die Sicherung der Öllieferungen in der Zukunft.

Aber teilten die USA und Großbritannien diese Auffassung? Für London ergaben sich aus den verschiedenen Debatten über die eigene strategische Zukunft in der Nahostregion im Allgemeinen und die Golfregion im Besonderen durchaus neue Perspektiven auf die iranische Rolle. Allerdings führte der eigene Niedergang nach Suez im arabischen Zentrum der Region zuvorderst zu einer Aufwertung der früheren Peripherie im Golf und auf der arabischen Halbinsel. Die Versteifung auf die Restposition und das Festhalten an der eigenen militärischen Rolle im Golf verringerte in der Konsequenz die Bereitschaft zur Kooperation mit Iran. Gegenüber Talbot erwähnten die Briten das Interesse des Schahs an Gesprächen, gestanden aber zugleich ein, dass London nicht gedenke ,[...] to get into a consultative capacity with him

${ }^{181}$ Vgl. MemCon, 13.4.62, FRUS 61-63, XVII, S. 610-22. 
over Gulf problems. “182 Diese Auffassung blieb dominant bis zum Ende der 1960er Jahre und der Ankündigung des britischen Rückzugs „East of Suez“, die dann eine Verständigung mit Teheran über die zukünftige Ordnung des Golfs unumgänglich machte. Bis zu diesem Zeitpunkt allerdings blieb man in diesen Fragen auf Distanz zu Iran, trotz des gemeinsamen Bündnisses im Rahmen der CENTO-Allianz, aufgrund der letzten Endes vorrangigen und zuvorderst ökonomischen Interessen Londons entlang der Südküste des Golfs. So bezeichnete ein internes Papier noch 1966 jede Steigerung des iranischen Einflusses im Golf als „disastrous“. 183

Selbstverständlich war sich auch die Kennedy-Administration der iranischen Ambitionen im Persischen Golf bewusst. Die oben behandelte Polk-Studie ging explizit auf diese ein. Die Dominierung des Golfs durch eine mächtige und feindselig gesinnte Macht zu verhindern, sei ein Kardinalprinzip iranischen strategischen Denkens. Und hinter dieser eher defensiven Grundkonstante schlummerten eigene Ambitionen, die Region selbst zu dominieren und die Kontrolle über die Ölfördergebiete entlang der Südküste zu erlangen. Die Aufrechterhaltung zweifelhafter eigener territorialer Ansprüche auf Bahrain und einige Inseln im Golf - „based on vague historical and demographic grounds“ - diene letzten Endes der Durchsetzung solcher Ziele im Anschluss an den erwarteten Abzug der Briten. Während der Kuwaitkrise war Iran demonstrativ dem Emirat beigestanden und hatte sogar Marineeinheiten vor die kuwaitische Küste entsandt, offenbar die Entschlossenheit Mohammad Rezās dokumentierend, keinen Machtzuwachs des irakischen Rivalen um die regionale Vorherrschaft in der Zukunft zuzulassen. ${ }^{184}$ Die herausragende Bedeutung des Golfs für die iranische Grand Strategy in der Region zeige sich zudem an der Tatsache, dass der Schah die Formulierung der Golfpolitik dem SAWAK und nicht dem Außenministerium übertragen habe. Einer früheren Fassung von Polks Studie zufolge war das Hauptaugenmerk iranischer Golfpolitik auf die Zukunft der britischen Klientelstaaten der Vertragsküste, sowie auf Bahrain, Katar und den Oman gerichtet. Daher versuche Teheran nun, die Scheichs mit Blick auf die Zukunft zu umgarnen und sie von der Notwendigkeit zu überzeugen, dass allein Iran ihre Herrschaft angesichts der Ausbreitung panarabischer und nasseristischer Strömungen garantieren könne. Zudem versuche man Großbritannien von den Vorzügen einer ,transition from British to Iranian dominion as being preferable from the British viewpoint to turning over the area to anti-British powers“ $\mathrm{zu}$

\footnotetext{
${ }^{182}$ London \#A-2919, 31.5.63, POL UK-US, CF 63; vgl. Harrison Letters, 11.12.62/4.2.63, FO 371/168632, TNA:PRO.

${ }^{183}$,Anglo-American Talks on the Middle East, UK Brief No. 5: Iran‘, Februar 1966, FO 371/186422, TNA:PRO. 184 Report ,Oil and Interdependence‘, S. 342, Anm. 162, S. 14f.; im Falle eines Abzugs standen die iranischen Chancen auf eine Vorreiterrolle recht gut. Die Polk-Studie erwähnte die konventionelle Überlegenheit Irans mit einer Armee in Größe von 171.000 Mann im Vergleich zu der nur 70.000 Mann starken und durch die internen Auseinandersetzungen mit den Kurden zudem stark geschwächten irakischen Armee. Saudi-Arabien mit nur knapp 15.000 Mann starker regulärer Armee war im Grunde zu vernachlässigen. Die iranische Marine sei stärker als die irakischen und saudischen Seestreitkräfte zusammen. Ende 1961 bestätigte der Schah gegenüber dem CIA Station Chief die Inbetriebnahme eines starken Senders in Ahwāz für die Übertragung arabischsprachiger Propagandasendungen an die Bevölkerungen Iraks und der Golfstaaten. Zudem stände ein zweiter Sender für kurdischsprachige Sendungen kurz vor der Fertigstellung. Vgl. Yatsevitch Memo, 14.12.61, encl. to Tehran \#293, 20.12.61, 788.11/12-2061, CF 60-63.
} 
überzeugen. ${ }^{185}$ Allerdings müsse man sich auch in Teheran trotz der Größe der schiitischen und iranisch-stämmigen Minderheiten auf der arabischen Seite des Golfs bewusst sein, dass ein Anschluss der doch vorwiegend arabisch geprägten Scheichtümer an Iran nicht realistisch sei. Die Endfassung der Polk-Studie schlussfolgerte dementsprechend: „[...] the Iranians would aspire to hegemony but would be satisfied with some degree of influence." Zwar anerkannte man von amerikanischer Seite die grundsätzliche Legitimität der iranischen Sicherheitsinteressen am Golf, hatte aber bislang jede Umorientierung der Militärplanungen für die iranischen Streitkräfte auf zukünftige Machtprojektion im Golf trotz Drängen Teherans konsequent abgelehnt. ${ }^{186}$ Die endgültige Fassung der Polk-Studie präferierte denn auch die graduelle Evolution der Golfscheichtümer hin zu einer Föderation und anschließender Unabhängigkeit nach Vorbild Kuwaits. Allenfalls sei ein Anschluss an Saudi-Arabien vorstellbar. $^{187}$

Anfang der 1960er Jahre waren somit die langfristigeren amerikanischen Planungen zur zukünftigen Sicherung der Stabilität in der Golfregion rudimentär. Obgleich man bis Ende der Dekade eine Auflösung des britischen Primats bzw. einen Abzug Großbritanniens erwartete, stellte man keine realistischen Überlegungen zu alternativen Sicherheitsarchitekturen oder zur Ausweitung der eigenen Rolle an. Es gab keine behördenübergreifende Anstrengung, neue Strategien für die Zukunft zu formulieren. Die spärlichen Überlegungen, vorwiegend die verschiedenen Studien Polks, sahen zwar eine bedeutsamere Rolle Irans in zukünftigen Sicherheitsstrukturen voraus, waren aber dennoch weiterhin eher geprägt von der in dieser Zeit dominierenden Sichtweise, derzufolge das Land in erster Linie mit seinen internen Problemen beschäftigt sei. Angesichts der weit verbreiteten Vorstellungen über den Mangel an Stabilität dachte kaum ein amerikanischer Offizieller ernsthaft über eine zukünftige stabilisierende Rolle Irans in der Sub-Region des Golfs nach. Somit kann auch die zweite Spielart des ,regionalen Arguments“ zur Erklärung der Aufgabe des reformistischen Interventionismus nicht überzeugen: Eine Korrelation zwischen dem Wandel der amerikanischen Regionalpolitik und der weitgehenden Einstellung reformistischer Bemühungen gegenüber Iran lässt sich empirisch

\footnotetext{
${ }^{185}$ Report ,The Persian Gulf in Middle Eastern Politics‘, o.D., encl. to Polk Memo to Persian Gulf Oil Planning Group, 5.6.62, Lot 69 D 121, S/P Records: 1962, Box 222; Polk zufolge war aus US-Sicht die Akzeptanz einer Föderation der Scheichtümer mit anschließender Unabhängigkeit die bevorzugte Lösung zu einem Anschluss an Irak, Saudi-Arabien und/oder einem pan-arabischen Großreich.

${ }^{186}$ Die Zusage der beiden Fregatten war ein erstes Vorzeichen für die Aufgabe dieser amerikanischen Position im Laufe der 1960er Jahre. In der ursprünglichen Version des MAP-Programms war bereits die Gewährung von \$20 Millionen für den Ausbau des Hafens von Bandar 'Abbās vorgesehen, ein lange vom Schah erwünschter Schritt als zukünftiges navales Zentrum für die Machtprojektion im Golf und für die Kontrolle der Straße von Hormus. Allerdings waren die amerikanischen Motive hier in erster Linie in dem Bestreben zu suchen, eine alternative Handelsroute für Afghanistan zu öffnen, um eine weitere Orientierung Kabuls nach Moskau zu verhindern. Vgl. Hamilton Memo, o.D. [8. bzw. 9.3.62], FRUS 61-63, XVII, S. 512-6; seit 1956 fanden jährlich kleinere britischamerikanisch-iranische Seemanöver statt. Vgl. Komer Memo, 2.2.62, ,Staff Memoranda, Robert Komer 2/62', NSF, M\&M, Box 322, JFKL.

${ }_{187}$ Anders als im Persischen Golf gab es allerdings in Washington eine begrenzte Bereitschaft, Iran eine regionale Rolle im Sinne westlicher Interessen im Hinblick auf die CENTO-Staaten und bezüglich Afghanistans zuzugestehen, ein Trend, der sich in den Folgejahren angesichts der internen Krisen dort sowie in der Türkei und in Pakistan noch verstärken sollte. Vgl. Halla Memo, 6.2.61, ,Iran (1)‘, WHO, NSCS, Special Staff File Ser., Box 4, DDEL; Position Paper, ,Afghan-Iran Relations', 14.4.61, ,Ninth CENTO Ministerial Session', Records of the Director (NE): 1958-1963, Box 5, RG 59, NACP.
} 
kaum belegen.

Wenn sich schon im Kontext der Formulierung der amerikanischen Regionalstrategie keine Anhaltspunkte finden lassen, welche den Abbruch des Reformprojekts in Iran erklären könnten, so ergibt sich vielleicht ein (6) globaler Konnex zur Implementierung des seitens der KennedyAdministration bereits frühzeitig propagierten Kurswechsels im militärischen Bereich im Sinne einer ,flexible response“ auf die sowjetische Bedrohung. Wie in den vorhergehenden Kapiteln ausführlich dokumentiert, fanden auch bezüglich des iranischen Falls eine Reihe von internen Debatten über den Stellenwert des Landes im Gesamtrahmen der westlichen Verteidigungsposition entlang der sowjetischen Peripherie statt. In den Diskussionen über die Reform der Militärhilfe im Sommer 1961, im Kontext der angespannten Beziehungen zu Moskau im Oktober 1961, und dann wieder während der Vorbereitung des Schahbesuchs im Frühling 1962 wurden immer wieder der Zusammenhang zwischen interner Reformnotwendigkeit und Aufrechterhaltung einer glaubwürdigen militärischen Abschreckung gegenüber dem nördlichen Nachbarn betont. ${ }^{188}$ Nahezu alle Offizielle, die maßgeblich an der Politikformulierung gegenüber Iran beteiligt waren, befassten sich in diesen Kontexten intensiv mit Verteidigungsfragen im globalen Kontext. Erst nach und nach wurde man sich auch der militärischen Dimension bewusst, die im Kontext der frühen Task Force-Beratungen noch ausgeklammert worden war. Detaillierte militärische Studien ließen im Laufe des Jahres 1961 bald keinen Zweifel mehr, dass die anfangs angedachte größere US-Rolle in der Verteidigung der Peripherie - eine zentrale Voraussetzung dafür, eine Neuorientierung zugunsten innerer Reformen weg von den militärischer Aufrüstungsbestrebungen zu ermöglichen - nicht umsetzbar war, bzw. die Globalstrategie der USA im Ganzen in Frage gestellt hätte. ${ }^{189}$ Alternativen $\mathrm{zu}$ einer solchen aufgewerteten US-Rolle wie z.B. eine neuerliche Schwerpunktsetzung auf regionale kollektive Verteidigung, also auf das CENTO-Bündnis, wurden angesichts der bekannten strukturellen Schwächen dieses Ansatzes nicht ernsthaft in Erwägung gezogen. ${ }^{190}$ Der Policy Planning Council beteuerte im August 1962 ein weiteres Mal, dass „the reasons for the U.S. not joining CENTO still remain overriding.“191 Ebenso

\footnotetext{
${ }^{188}$ Vgl. oben S. 280f., 284-8, 295-8.

189 Alle diese Debatten kamen allerdings zu keinem endgültigen Ergebnis bzw. zu keiner finalen Politikempfehlung. Das Pentagon widersetzte sich zudem Entscheidungen, die eine Neujustierung der Rolle Irans im globalen Verteidigungskonzeption notwendig gemacht hätten. Vgl. MemCon, 12.3.62; tatsächlich hatte sich inzwischen auch die empfohlene Reaktion auf die interne Bedrohung verschoben, von Reform auf Repression. Ein Strategiepapier des Policy Planning Council führte aus: „In sum, we think that the strategic threat to Iran is internal rather than external and that the principal task ahead is to structure, equip and train the Iranian forces to meet this threat."; Study ,A Strategy to Defend Iran', encl. to Ramsey Memo, 27.3.62, ,Iran“, Lot 69 D 121, S/P Records: 1962, Box 216; vgl. bereits früher Miner Memo, 9.11.61, ,16. U.S.-Iran Relations‘, NEA, GTI: RIAD, 1958-63, Box 3.

${ }^{190}$ Die noch im Kontext des Task Force Report eingerichtete State-Defense Working Group zur Einschätzung einer möglichen Aufwertung der US-Rolle innerhalb der CENTO-Allianz hatte im Januar 1962 einen Entwurf vorgelegt. Die Errungenschaften der Allianz selbst, gerade ihr militärischer Kern, wurden extrem negativ bewertet. Wie in den Jahren zuvor schon die JCS empfahl der Bericht den Beitritt zum Bündnis. Vgl. Report, ,Advancing U.S. Interests through CENTO‘, o.D. [Januar 1962], ,Near \& Middle East 1962‘, Lot 69 D 121, S/P Records: 1962, Box 222; die Einschätzung der Botschaft in Teheran zur iranischen Sicht auf CENTO in Rockwell Letter, 5.10.61, Lot 65 D 365, NEA/CENTO Records, 1956-65, Box 1.

${ }^{191}$ Rostow Memo, 2.8.62, ,Near \& Middle East 1962‘, Lot 69 D 121, S/P Records: 1962, Box 222; ein CENTOBeitritt allein, so die Einschätzung der Policy Planner, könne die Unklarheit bezüglich der US-
} 
wenig war man bereit, den exzentrischen Ideen Paul Nitzes zur Lösung des Dilemmas mit seinem Befürworten taktisch-nuklearer Verteidigung des Landes inklusive weitreichender nuklearer Teilhabe $\mathrm{zu}$ folgen. ${ }^{192}$ Die Kennedy-Administration verschob daher eine Grundsatzentscheidung über das CENTO-Bündnis und setzte im Kern die Eisenhower-Politik fort. $^{193}$

Nicht nur die zeitliche Parallelität zu den Debatten bezüglich fortgesetzter reformistischer Intervention legt hier einen engeren Zusammenhang nahe. Auch inhaltlich sind die militärstrategischen Debatten dieser Zeit mit den Fragen der Möglichkeit erzwungener Transformation Irans von außen eng verknüpft. In den Akten ist z.B. die Ernüchterung innerhalb der Administration zu jenem Zeitpunkt spürbar, als man sich der militärstrategischen Folgen einer Neutralisierung Irans im Kontext einer denkbaren Machtübernahme der Nationalen Front bewusst wurde - eine solche hätte die rechtzeitige Einführung amerikanischer Truppenkontingente bzw. der bislang als zentral erachteten taktischen nuklearen Kapazitäten in Iran wohl unmöglich gemacht. Somit gewährleistete nur eine pro-westliche Führung in Teheran die Möglichkeit, eine Verteidigung des Landes im Ernstfall überhaupt zu versuchen.

Allerdings waren diese Debatten überwiegend theoretischer Natur: Kaum jemand erwartete einen isolierten Angriff der Sowjetunion auf Iran. Kampfhandlungen in dieser Region wurden, wenn überhaupt, immer im Kontext einer globalen Konfrontation der Supermächte unmittelbar im Vorfeld eines Dritten Weltkriegs erwartet. In einem solchen Kontext waren militärische Detailfragen bezüglich peripherer Länder wie Iran eher zweitrangig. Zudem sahen die New Frontiersmen nicht direkte sowjetische Militäraktionen als zentrale Bedrohung der westlichen Position, sondern eben die Reorientierung Moskaus auf Subversion und Unterstützung nationaler Befreiungsbewegungen. Frühere Einbußen der westlichen Position in der Nahostregion waren zudem allesamt Folgen interner Verwerfungen und Machtwechsel in den jeweiligen Staaten und eben nicht Resultate direkten sowjetischen Eingreifens. Somit waren die dominanten Bedrohungsvorstellungen der Administration und das institutionelle Gedächtnis in der Tat eher mit einer Politik reformistischer Intervention vereinbar als mit einer primär konventionell-militärischen Sichtweise, die man ohnehin als Neuauflage des

Sicherheitsgarantien nicht beseitigen. Konkrete militärische Maßnahmen bzw. bilaterale Vereinbarungen seien weitaus besser geeignet. Ein Beitritt der USA könne zudem die Hinwendung Afghanistans und Indiens zu Moskau beschleunigen bzw. israelische Forderungen nach stärkerer US-Unterstützung auslösen, die zudem vom amerikanischen Kongress unterstützt würden.

${ }^{192}$ Vgl. oben S. 296ff.; auch Komer hatte sich im März 1962 des Problems angenommen und ein weiteres Mal im Grundsatz seine Position vom Herbst 1961 bekräftigt. Er sah ebenfalls die eigentliche Hauptbedrohung in „,indirekter Aggression“. Dagegen seien als Maßnahmen nicht allgemeine Kriegsplanungen oder der Ausbau der iranischen Streitkräfte effizient, sondern vielmehr Programme zur Stärkung der „Aufstandsbekämpfung“ sowie „Programs designed to promote local stability, development, reform in a manner which will prevent revolutionary situation from arising in the first place."; Komer Memo, 24.3.62, ,Iran General 3/1/62-3/26/62', NSF, Countries, Box 116, JFKL; zur weiterhin ungeklärten Frage der Nationalität eines Commander-in-Chief für CENTO vgl. MemCon, 13.4.62, FRUS 61-63, XVII, S. 610-22.

${ }^{193}$ Vgl. Policy Directive, PD/NEA-1, 23.2.62, ,Department of State, Policy Directives, NEA [...] $]^{‘}$ NSF, D\&A, Box 288, JFKL; INR im State Department stellte lapidar fest: „CENTO has not posed serious problems for us since Iraq left the alliance.“; Hughes Memo [INR-62], 6.3.62, FRUS 61-63, VII-VIII-IX Suppl., Dok. 263. 
gescheiterten Ansatzes der Eisenhower-Administration gegenüber der Dritten Welt betrachtet hätte. Die eingeforderte Flexibilität einer amerikanischen Antwort im Falle einer tatsächlichen militärischen Bedrohung durch die UdSSR wäre somit im Ernstfall durch die Möglichkeit horizontaler Eskalation realisiert worden, also durch Gegenmaßnahmen an geographisch günstigeren Positionen entlang der sowjetischen Peripherie. Auch der globale Konnex überzeugt nicht wirklich als determinierender Faktor für die Erklärung der Wende in der amerikanischen Iranpolitik.

Augenfällig ist, dass der reformistische Schwung der Kennedy-Administration zu eben jenem Zeitpunkt an Kraft verlor, als der bürokratische Widerstand gegen diesen Kurs vorerst gebrochen schien, und zudem bevor man überhaupt in der Lage war, die Wirkung der ersten reformerischen Maßnahmen der Amini-Regierung bzw. der eigenen offenen wie verdeckten Einflussnahme zu bewerten. Daher ist naheliegend, die Ursache für den Politikwechsel in einer Veränderung der breiteren Grand Strategy der Administration zu suchen, die dann auf die eigentliche Iranpolitik zurückwirkte. Angesichts des Fehlens zentraler Strategiefestlegungen aufgrund des vorherrschenden „Ad-hocismus“ der New Frontier ist die Nachverfolgung einer solchen Veränderung kein leichtes Unterfangen. Wie in Kapitel IV.1. beschrieben, verengte sich die frühe Herangehensweise der Kennedy-Administration gegenüber den Staaten der Peripherie rasch von einem breiten reformistischen Ansatz - gleichermaßen Entwicklung und Sicherheit betonend - hin zu einer einseitigen Konzentration auf sicherheitspolitische, militärische, polizeiliche und nachrichtendienstliche Aspekte der Aufstandsbekämpfung. Ambitionierte Zielsetzungen wie die Schaffung einer neuen politischen Synthese in Iran oder vergleichbare Projekte, z.B. in Südkorea oder gerade auch in den Staaten Südamerikas im Rahmen der „Allianz für den Fortschritt“, wurden offenbar im gleichen Zeitraum wie das „IranExperiment" abgeschwächt bzw. ihres reformistisch-interventionistischen Kerns beraubt. ${ }^{194}$ Der genaue Zeitpunkt der jeweiligen Wende in den verschiedenen reformistischen Projekten entlang der Peripherie ist dabei im Einzelnen aufgrund der Vielschichtigkeit der Ansätze und der jeweiligen spezifischen Gegebenheiten nur schwer auszumachen und ist auch nicht Gegenstand dieser Untersuchung. Interessant ist aber die große Ähnlichkeit der Herausforderungen, welche die Kennedy-Administration zu meistern hatte. Vergleichbar zur grundsätzlichen Frage der Vereinbarkeit von monarchisch-autokratischem System in Iran mit

\footnotetext{
${ }^{194}$ Eine ähnliche Kehrtwende in der US-Politik lässt sich ungefähr in diesem Zeitraum z.B. an der Frage der nicaraguanischen Wahlen und der Haltung gegenüber der Somoza-Diktatur illustrieren. Vgl. GAMBONE, Capturing the Revolution, S. 35ff., ähnlich wie in Iran auch hier die Rolle der Counterinsurgency-Konzepte. Siehe ebd., S. 55-62; parallele Entwicklungen zeigten sich u.a. in der Politik gegenüber Indonesien, Paraguay und Bolivien. Für die Entwicklungen in Lateinamerika allgemein vgl. RABE, Most Dangerous Area, S. 99-172; Südkorea nach dem Rücktritt von Syngman Rhee 1960 ist ein anderes eindrückliches Beispiel. Die US-Politik gegenüber Südkorea ähnelte in vielem derjenigen gegenüber Iran. Parallel zur Iran Task Force wurde auch eine Task Force für Südkorea eingerichtet. Die anfangs distanzierte Haltung gegenüber der mit einem Staatsstreich gegen die (demokratische) Zweite Republik im Mai 1961 an die Macht gekommenen Militärdiktatur unter Park Chung Hee wurde bereits Ende 1961 aufgegeben. Vgl. BRAZINSKY, Nation Building in South Korea, S. 118-25; dabei muss beachtet werden, dass der US-Einfluss in Ostasien und gerade in Südkorea (aber auch in Taiwan und den Philippinen) immer bestimmender war als derjenige in nahöstlichen Staaten. Die Entwicklungen der US-Politik gegenüber der autokratischen Führung unter Kaiser Haile Selassie in Äthiopien gleichen denen in Iran in verblüffender Weise. Vgl. MARCUS, Ethiopia, S. 170-83.
} 
der Notwendigkeit sozialer und wirtschaftlicher Reformen thematisierten US-Offizielle in anderen geographischen Abteilungen innerhalb des State Department die Problematik, dass ein sozio-ökonomischer Modernisierungsprozess und das Generieren von Wachstum mit dem langfristigen Ziel, wirtschaftlich erfolgreiche, gerechtere und pro-westliche Gesellschaften in der Peripherie zu kreieren, im Grunde unerreichbar blieb, solange man eine grundlegende Revision der politischen Systeme aussparte. Auch hier stellte sich die Frage, welches Ausmaß der reformistische Interventionismus annehmen musste, um erfolgversprechend $\mathrm{zu}$ sein. ${ }^{195}$ Eine Diskussion der wichtigsten Planer der Alianza der Kennedy-Administration mit Außenminister Rusk im Februar 1962 befasste sich mit eben dieser Grundsatzfrage bezüglich Lateinamerikas und ihren Auswirkungen auf das zentrale Ziel der Reformanstrengungen, ,[...] to encourage the growth of reasonably stable governments capable of absorbing reform and change, secure from both the extreme Left and the extreme Right."196

Der Bericht einer Survey Group des Bureau of the Budget vom August 1962 über den Zustand der Alianza bis dato illustriert eindrücklich die vielen Parallelen zu den Reformbemühungen in Iran. Es trägt die Handschrift von Kenneth Hansen, dem hochrangigsten Mitglied der Survey Group, die im Sommer 1962 für mehrere Wochen verschiedene südamerikanische Länder bereiste, der ja auch im iranischen Fall auf Seiten der reformistischen Interventionisten eine prononcierte Rolle gespielt hatte. Die Vielschichtigkeit der amerikanischen Strategie im Rahmen der Alianza widerspiegelnd, differenzierte der Bericht zwischen drei Stufen amerikanischer Einmischung in den Empfängerstaaten, die im Kern das Ausmaß der als notwendig empfundenen Intervention in die politischen, wirtschaftlichen und sozialen Realitäten für das Erreichen der angestrebten Transformation derselben beschrieben. Neben einem passiv ausgerichteten ,program of detachment“ und einem im Grunde der tradierten Eisenhower-Politik nachempfundenen ,holding the line“-Ansatz definierte der Bericht noch eine dritte Stufe, die als Politik der „massive intervention“ charakterisiert wurde. Die Stufen waren idealtypisch konstruiert und es wurde ausdrücklich mit hybriden Umsetzungen in der Realität gerechnet. „Massive Intervention“ zielte letztlich auf eine von Außen induzierte und unterstützte fundamentale Transformation der Zielgesellschaft. Ausgehend von der Annahme, dass die interne Blockierung rapider Modernisierungsanstrengungen aufgrund von historischen Pfadabhängigkeiten und der Trägheit infolge der tradierten Dominanz starker Interessengruppen nur durch Druck und Einfluss externer Macht überwunden werden könne, empfahl das Papier die Involvierung der USA in sämtliche wirtschaftliche Sektoren eines Landes, in die Finanz- und Währungspolitik und auch sonst in sämtliche Bereiche, die sich mit Ressourcenallokation im Allgemeinen befassten. Dabei ging es nicht alleine um die effiziente

${ }^{195}$ Die Grundsatzfrage ließ sich auf das Verhältnis zwischen political development und social and economic development zuspitzen. Dieses Grundproblem ähnelte dabei stark den Debatten im Kontext der Iran Task Force. ${ }^{196}$ MemCon, 13.2.62, FRUS 61-63, XII, S. 91; auf das Zitat folgt die Feststellung, die Zielsetzung in der westlichen Hemisphäre sei weitreichender als in Asien, wo man auf die Schaffung von ,neutral countries capable of maintaining their own stability“" abziele. Iran ähnelte, was die langfristigen Zielsetzungen anging, daher eher den lateinamerikanischen Staaten als anderen asiatischen, da eine Neutralität Irans in der Zukunft inakzeptabel erschien. 
Umsetzung der spezifisch von eigener Seite geförderten und priorisierten Reformmaßnahmen und Entwicklungsprojekte, sondern ausdrücklich um ,programs and projects which are not directly attributed to our assistance. “197 Für eine solche ,aktivistische Rolle“ mit dem Ziel ,[...] to bring about radical changes in political and economic processes" sei es notwendig, erhebliche Risiken einzugehen:

In the process, the U.S. agencies would need to develop the capability of carrying out this activity through direct involvement in the key processes of decision-making in the countries assisted, and develop means - independent of those of direct government-to-government contacts - to influence public opinion and create the essential public pressures for reform. Without such pressures it is not likely that the governments will move vigorously toward reform. ${ }^{198}$

Der Bericht gestand ein, dass eine solche Politik permanente Einmischung als ,indiscriminate and arbitrary interference or gross infringement of sovereignty" der Zielstaaten missverstanden werden könne, zumal man danach strebe ,[...] in strategic sectors and for various decisions we shall somehow obtain a strong voice in resolution of public issues." Allerdings müsse man sich eingestehen, dass die Alianza selbst mit ihren weitreichenden Zielsetzungen im Grunde ein ,interventionist device“ sei. Entschlossene reformistische Politik könne nicht in einer „atmosphere of hypersensitivity“ florieren. ${ }^{199}$

Der Survey Report zur Alianza gibt geradezu idealtypisch die Probleme und Hindernisse der reformistischen Intervention in Iran wieder und illustriert die Ähnlichkeit dieser parallelen Anstrengungen amerikanischer Strategen zur Immunisierung der Peripherie gegenüber revolutionärer Gefahren in verschiedenen geographischen Umgebungen. Der Report warnte, dass eine solch umfassende „Strategie der massiven Intervention“ nur in jenen Fällen tragfähig sei, in denen die politischen und wirtschaftlichen Voraussetzungen für ,rapid and sustained development“ gegeben seien. Falls eine derartige „politische Synthese“ - eine weitere sprachliche Parallele zu den Diskussionen über Iran - nicht bestehe, sei eine solche Strategie kontraproduktiv für die eigenen Interessen. ${ }^{200}$ Harold Saunders, Komers engster Mitarbeiter im Weißen Haus, lobte den Survey Report für die beispielhafte Darlegung für ,[...] our failure to marry economic and political strategy." ${ }^{201}$

Analog zu den Debatten über die Iranpolitik stand auch das Hilfsprogramm für Lateinamerika vor dem Dilemma, dass die als unabdingbar angesehenen sozialen und wirtschaftlichen Veränderungen nur bei parallel vonstatten gehender politischer Reform erfolgversprechend

\footnotetext{
${ }^{197}$ Bureau of the Budget Staff Report, 7.8.62, siehe oben S. 321, Anm. 96.

${ }_{198}$ Ebd., S. 3; allerdings gestand der Report auch ein, dass die entsprechenden personellen Kompetenzen in den eigenen Vertretungen fehlten. So sei der IMF das „major external wheelhorse striving for financial discipline“ und nicht etwa US-Berater. Ebd., S. 12.

${ }^{199}$ Ebd., S. 2 \{Hervorheb. v. Verf.\}.

200 Ebd., S. 3, 4.

${ }^{201}$ Saunders Memo, 25.9.62, ,National Security Problems, 1964 [White House Memoranda]', NSF, RWK, Box 438, JFKL.
} 
waren. Letztere aber - zumindest in einer für die amerikanischen strategischen Interessen akzeptablen Form - war letztlich nur unter Einsatz eigener verdeckter wie offener Mittel erreichbar. Es ging also auch im Kontext der Alianza um die Frage des Ausmaßes und der Zielrichtung des reformistischen Interventionismus. In Lateinamerika entsprachen die traditionellen Oligarchien und das meist mit diesen verbündete Militär der Rolle des Hofs und seiner politischen Unterstützungsbasis in Iran. Die Entwicklung der „Allianz für den Fortschritt" glich dabei augenfällig der parallelen reformistischen Task Force-Politik in Iran. Anfangs ambitionierte reformistische Zielsetzungen, politische Transformationen grundsätzlich einbeziehend, verwässerten rasch zu einer kraftlosen Weiterführung zunehmend bescheidener entwicklungspolitischer Zielsetzungen. Statt Reformkoalitionen als politische Basis für die Transformation der Gesellschaft anzustreben - das Äquivalent in Iran war die neue politische Synthese -, fiel man rasch in alte Muster der Eisenhower-Zeit zurück. Fatal für die Zukunft war aber die parallele Überbetonung der militärischen Aspekte der eigenen Strategie, der Dominanz der Subversionsängste und die Sorge vor weiteren „Kubas“, die eine Schwerpunktsetzung auf Aufstandsbekämpfung zur Folge hatten. Sicherheit wurde gegenüber Entwicklung bald priorisiert. Da diese Gruppe eine „extremely important strategic position“ einnähme, drängte Kennedy im September 1961 selbst auf eine Reihe von Maßnahmen ,,[...] to increase the intimacy between our Armed Forces and the military of Latin America." ${ }^{202}$ Die Rückbesinnung auf die herausgehobene Stellung des Militärs als eigentlichem Schutzwall gegen kommunistische Subversion brachte zugleich eine Aufgabe des breiteren Reformismus mit sich. Zwar wird in den Dokumenten mitunter die Hoffnung geäußert, jüngere Offiziere könnten in Zukunft zu Trägern sozialer und wirtschaftlicher Reformmaßnahmen werden. Doch gab die tradierte Haltung des lateinamerikanischen Militärs als Bewahrer des Status Quo kaum Anlass für derlei Erwartungen. Ähnlich wie im iranischen Fall bedeutete die Stärkung der traditionellen Machtzentren in den lateinamerikanischen Staaten, zuvorderst des Militärs, eine Aufgabe der breiten Reformprogramme, wenngleich keine vollständige Einstellung der Reformbemühungen an sich. Fast zeitgleich zur oben geschilderten Klage Robert Komers vom Oktober 1962 über die Aufgabe der Task Force-Politik in Iran, kritisierte Arthur Schlesinger Jr. die ,recrystallization of elements of the Eisenhower policy within the framework of the

\footnotetext{
${ }^{202}$ Kennedy Memo [NSAM N ${ }^{\circ 88}$, Training for Latin American Armed Forces'], 5.9.61, FRUS 61-63, XII, S. 180; fast zeitgleich warnte Under Secretary Bowles vor möglichen Folgen der bedingungsfreien Militärhilfe an lateinamerikanische Staaten. Man müsse Sorge tragen, dass , , [... this aid is not to be used to deter legitimate popular expressions of aspirations for greater social justice and political freedom."; Bowles Memo, ebd., S. 190.; ein weiterer von Kennedy selbst in Auftrag gegebener Bericht zur Unterstützung lateinamerikanischer Streitkräfte vom Februar 1962 betonte, die Stärkung der Streitkräfte gegen kommunistische Subversion à la Castro brächte zugleich „hazardous political consequences“" mit sich. Es sei aber unumgänglich, dass „the United States must be the paramount foreign military influence in Latin America.“; alle Zitate aus Battle Memo, 5.5.62, ebd., S. 214-7; LATHAM, Modernization as Ideology, S. 69-108, konzentriert sich in seiner Schilderung der Alianza auf die hochtrabende Rhetorik der New Frontiersmen und insbesondere Walt Rostows, schildert aber so gut wie nicht die Umsetzung der Politik durch die Administration, insbesondere die frühe „militärische Wende“. Auf diese Weise umgeht er die politischen Folgen dieser Wende, nämlich Beschränkungen des Reformwillens und politische Repression der Mittelschichten, der vorgeblich zentralen Träger des Modernisierungsprozesses. Auf diese Weise kann Latham den Widerspruch umgehen, dass die angeblich von ideologischem Modernisierungsdenken angeleitete US-Politik zentrale Bestandteile einer solchen modernistischen Transformation der Zielgesellschaften früh aufgab.
} 
Alliance for Progress“. 203

Wie im Fall Irans lässt sich auch hinsichtlich der Alianza tatsächlich ein früher Wendepunkt in der US-Politik im Herbst 1961 und zur Jahreswende 1961/62 identifizieren. Das fortgesetzte Drängen Kennedys auf eine Priorisierung der Beziehungen zu militärischen Kreisen in Lateinamerika ist dabei das Äquivalent zur Rückbesinnung auf die iranische Monarchie als wichtigster Stabilitätsanker in Iran. Beide bedeuten keine grundsätzliche Aufgabe der amerikanischen Reformwünsche, sondern vielmehr die Einzäunung angestrebter sozioökonomischer Transformationen innerhalb geerbter und aus anti-kommunistischem Blickwinkel bewährter politischer Konstellationen. Der Widerspruch war dabei auch den Zeitgenossen durchaus bewusst, negierte doch der implizite Verzicht auf politische Umgestaltung die vorgebliche Zielsetzung auf ein zukünftiges Konvergenzziel westlicher wirtschaftsliberaler und freiheitlich-demokratischer Systeme. Zudem bedeutete der Verzicht auf politische Transformation das Weiterbestehen der auch wirtschaftlich dominierenden Gesellschaftsgruppen in herausgehobenen politischen Positionen, was wiederum dem Reformeifer auf sozio-ökonomischer Ebene enge Grenzen setzte.

Im Falle der Alianza mit Lateinamerika ist die Intensivierung der Aufmerksamkeit für die herausgehobene (politische) Rolle des Militärs und den Themenbereich interne Sicherheit/Aufstandsbekämpfung im letzten Quartal 1961 augenfällig. Die JCS reagierten auf das Drängen des Präsidenten im November 1961 mit einem ausführlichen Bericht über den möglichen Beitrag des US-Militärs für eine erfolgreiche Umsetzung der ambitionierten Ziele der Allianz. Hauptziel war eine stark intensivierte Kooperation mit lateinamerikanischen Militärs, zuvorderst gerichtet auf eine Umorientierung der militärischen Ausrichtung von zwischenstaatlich-konventionellen Konflikten hin zu Bekämpfung von Subversion und Insurgenz im Inneren. Diese Neuausrichtung, einhergehend mit umfangreichen Trainingsmaßnahmen und der Lieferung von entsprechender Ausrüstung, hatte augenfällig weitreichende politische Konsequenzen. Der JCS-Bericht betonte daher vorauseilend, es ginge nicht zuletzt um die Vermittlung der eigenen Werte der zivilen Kontrolle der Streitkräfte, angesichts der historischen Erfahrungen Lateinamerikas eine kaum ernstzunehmende Ergänzung. ${ }^{204}$ Im Dezember weitete Kennedy die vorhergehende Instruktion in NSAM N${ }^{\circ} 118$

\footnotetext{
${ }^{203}$ Schlesinger Jr. Memo, 15.10.62, FRUS 61-63, XII, S. 110 \{Hervorheb. v. Verf.\}; unter anderem kritisierte Schlesinger die Akzeptanz der Militärcoups gegen demokratisch gewählte Regierungen durch die KennedyAdministration. Ein Jahr später wandte er sich explizit gegen die sogenannte Martin-Doktrin und den Eindruck, man habe die progressiven Kräfte aufgegeben ,[...] and are now looking to military rule to produce progressive regimes."; Schlesinger Jr. Memo, 8.10.63, ebd., S. 150ff., hier 151; Bowles hatte bereits im Oktober 1961 vor einer Aufgabe der ambitionierten Ziele gewarnt. Mexico City \#SECUN 26, 19.10.61, FRUS 61-63, XII, S. 67; zur weiteren Entwicklung hin zu Vertiefung verdeckter Operationen gegen links gerichtete Regierungen und zur Absicht einer öffentlichen Verkündigung einer Kennedy-Doktrin, derzufolge die USA die Entstehung weiterer „Kubas“ in der Hemisphäre nicht hinzunehmen bereit wären, also der offenen Drohung mit Intervention, vgl. RABE, Johnson Doctrine, S. $52 \mathrm{ff}$.

${ }^{204}$ Vgl. JCS Memo, JCSM 832-61, 30.11.61, FRUS 61-63, XII, S. 197-202; das wird auch aus den Formulierungen des Berichts selbst offensichtlich: „Departure from the previously purely military roles by US and Latin American armed forces in implementing these recommendations will result in some resentment and criticism from certain power groups in Latin America."; ebd., S. 201; im Bericht ist auch wiederholt unverhohlen die Notwendigkeit
} 
noch aus und beauftragte die Ausarbeitung eines „Action Program“ und die Ausformulierung eines begleitenden politischen Ausführungsprogramms durch das State Department. ${ }^{205}$ Die Policy Guidance des State Department vom Februar 1962 definierte als Zielsetzung der angestrebten engen Kooperation mit lateinamerikanischen Streitkräften neben externer Verteidigung und effizienter Aufstandsbekämpfung und Aufrechterhaltung der inneren Sicherheit auch die Unterstützung sozialer und wirtschaftlicher Entwicklung und explizit die Bewahrung der demokratisch-konstitutioneller Ordnung. Allerdings wurde diese Weisung offenbar bewusst generisch gehalten - eine frühere Version hatte noch explizit vor ,hazardous political consequences“ infolge der Stärkung lateinamerikanischer Streitkräfte gewarnt. ${ }^{206}$ Auch hier drängt sich die Parallele zur evasiven Antwort der Iran Task Force auf NSAM №67 im gleichen Zeitraum auf. ${ }^{207}$

Die Parallelen hinsichtlich der graduellen Aufgabe früher reformistischer Ambitionen in Iran und Lateinamerika sind ein Beleg für die Stichhaltigkeit der Annahme, dass der eigentliche Hintergrund für diese Kehrtwende tatsächlich in einem Wandel der Grand Strategy der Kennedy-Administration zu suchen ist. Die hier vertretene These sieht die Wende in der Politik gegenüber Staaten entlang der Peripherie in Lateinamerika oder auch in Iran als Folge der raschen Militarisierung der anfänglich verfolgten breiten Reformpolitik der KennedyAdministration, insbesondere der rasch einsetzenden Dominanz von Strategiekonzepten aus dem Bereich der Counterinsurgency. Wie in Kapitel IV.1. geschildert, überlagerte in der Politikformulierung der New Frontiersmen das Prinzip „Sicherheit“ rasch die Prinzipien „Entwicklung“ und „Modernisierung“. Der Vorkämpfer für eine Kombination dieser Prinzipien innerhalb der Administration, Walt Rostow, war im Dezember 1961 vom Weißen Haus in den Policy Planning Council verschoben worden, eine auch symbolische Verbannung

erwähnt Kontakt zu den „up-and-coming officers“, den potentiellen ,emerging leaders“, aufzunehmen. Vgl. ebd., S. 199; JCSM 832-61 in DDRS: CK2349437380, S. 14; im dritten Teil des JCS-Berichts, nicht abgedruckt in der FRUS-Version, wird der Widerspruch auf den Punkt gebracht: „The crux of the problem today, however, is the profoundly conservative orientation of the military and the political groups with whom they have generally made common cause in the past, and a willingness of both these groups to use military force to resist even those changes which are approved by a majority of an electorate which is increasingly free to choose."; ebd., S. 68; parallel zu den JCS-Planungen erfolgte auch eine eingehende Untersuchung der internen Sicherheitssituation in den Staaten Südamerikas. Vgl. Report and Recommendations of the Washington Assessment Team on the Internal Security Situation in South America, 10.1.62, FRUS 61-63, XII, S. 202-9.

205 ,NSAM Number 118‘, 5.12.61, NSF, M\&M, Box 333, JFKL; vgl. Walsh Memo, 30.9.61, ,16. U.S.-Iran Relations‘, NEA, GTI: RIAD, 1958-63, Box 3.

${ }^{206}$ Vgl. Battle Memo [,NSAM N ${ }^{\circ} 118$-Participation of U.S. and Latin American Armed Forces in the Attainment of Common Objectives in Latin America'], 28.2.62, FRUS 61-63, XII, S. 221ff; tatsächlich ist im Dokument selbst von einem ,revised report“ die Rede. Zur früheren Version vgl. Battle Memo, 5.2.62, ebd., S. 214-7; die frühere Version macht klar, dass das State Department angesichts der internen labilen Situation in vielen südamerikanischen Staaten für eine nur partielle und länderbezogene Umsetzung der Strategie und den Verzicht auf einige der seitens der JCS vorgeschlagenen Maßnahmen plädiert hatte: ,We cannot afford to be identified with any step backward either to repressive dictatorship or military intervention in political life."; allerdings war zugleich davon die Rede, dass , ,...] the United States must be the paramount foreign military influence in Latin America“, um eine „favorable political orientation“ des lateinamerikanischen Offizierskorps sicherzustellen. Ebd., S. $215,216$.

${ }^{207}$ Die hier ausgearbeitete These in ihrer Gesamtheit überzeugend zu belegen, bedürfte es einer hier nicht $\mathrm{zu}$ leistenden, groß angelegten vergleichenden Untersuchung der Kennedy“schen Reformpolitik gegenüber verschiedenen Staaten und Regionen inklusive einer eingehenden Analyse der Politikformulierung auf dem Level der Grand Strategy. 
des reformistischen Aktivismus aus der Zentrale der strategischen Politikformulierung. ${ }^{208}$ Die verschiedenen Studien über die zu verfolgende eigene Grand Strategy gegenüber der Peripherie dagegen verstärkten den allgemein spürbaren und vom Präsidenten persönlich bevorzugten Trend hin zur Militarisierung, zur Betonung innerer Sicherheit und (militärischer) Aufstandsbekämpfung im Allgemeinen. Sowohl die JCS-Studie und der Abschlussbericht der NSC Counter-Guerrilla Warfare Task Force vom Dezember 1961 betonten die militärischen und sicherheitspolitischen Aspekte stark und ignorierten weitgehend zentrale Fragen der politischen und sozialen Ursachen für Unruhe und Aufstände in peripheren Gesellschaften. ${ }^{209}$ All dies mündete in die Schaffung des zentralen Lenkungsorgans der Special Group (CI) im Januar 1962 für die eigene periphere Strategie, deren Bezeichnung allein sinnbildlich für die einseitige Priorisierung der militärischen, polizeilichen und geheimdienstlichen Aspekte in der Politik gegenüber den ,neuen“ Staaten stand.

Wie in Kapitel V.1. geschildert, machte sich die verstärkte Konzentration auf Counterinsurgency-Konzepte auch innerhalb Irans bemerkbar. Infolge der Unruhen vom Januar 1962 an der Universität von Teheran hatte sich das Interesse an der Frage der inneren Sicherheit in dem Land bereits früh gemeldet, noch vor der offiziellen Aufnahme Irans im Juni 1962 in die Liste der Staaten, die für die Special Group (CI) prioritären Charakter hatten. ${ }^{210}$ Botschafter Holmes empfahl der Special Group (CI) im April die Ausdehnung der Aktivitäten der Public Safety Division in Teheran und die Intensivierung der Zusammenarbeit mit den iranischen Polizeikräften. ${ }^{211}$ Die bestehende amerikanische Beratermission hatte bereits eine

\footnotetext{
${ }^{208}$ Auch von seiner neuen Position aus setzte Rostow seinen Kreuzzug fort. So schlug er im Februar 1962 der Special Group (CI) die Gründung eines Institute of Modernization Studies vor. Vgl. Rostow Memo, 14.2.62, ,Special Group (CI) 1/1/62 Thru 7/31/62‘, Lot 68 D 451, SG (CI) Records, Box 1.

209 Vgl. POOLE, History of the JCS: VIII, S. 23-7, 225-31; Komer nutzte den Bissell-Report und Rostows Entfernung aus dem Weißen Haus, um die Special Group als zentrales Leitungsorgan für die Umsetzung der breiteren Strategie zu etablieren. Dies ist ein weiterer Beleg für die intendierte Distanzierung von Rostow und seinen (über-)ambitionierten Ideen. Vgl. Komer Memo, 12.12.61, FRUS 61-63, VII-VIII-IX Suppl., Dok. 250; ein interner Rückblick auf die Entstehungsgeschichte der Counterinsurgency-Konzeptionen in den frühen 1960er Jahren von Komers NSC-Mitarbeiter Harold Saunders fasste die verschiedenen Strömungen im ersten Jahr der Kennedy-Administration zusammen und konstatierte den Abschluss der „explanatory phase“ im Dezember 1961/Januar 1962. Kennzeichnend für das letzte Quartal von 1961 seien eine Zuspitzung auf Zusammenarbeit mit ausländischen Militär- und Polizeikräften sowie Zivilhilfe gewesen. Vgl. Saunders Memo, 10.3.64, ,CounterInsurgency Special Group 1964-1965-1966‘, NSF, FRWK, Box 15, LBJL.

${ }^{210}$ Maxwell Taylor hatte bereits im April 1962 von der Special Group (CI) eine Einschätzung der Lage der inneren Sicherheit in Iran angefragt. Vgl. Memo for Taylor, 17.4.62, ,SPECIAL [...] 7/31/62', Lot 68 D 451, SG (CI) Records, Box 1; für NSAM N 165 siehe FRUS 61-63, VIII, S. 307 n3.

${ }^{211}$ Vgl. Tehran \#A-268, ,Anti-Guerrilla Training and Civic Action (CA-2108)', 19.4.62, 788.5/4-1962, CF 60-63; Minutes Special Group Meeting, 26.4.62, ,SPECIAL [...] 7/31/62‘, Lot 68 D 451, SG (CI) Records, Box 1; insbesondere Komer hatte bereits während der frühen Debatten im Laufe von 1961 auf eine stärkerer Berücksichtigung der bereits bestehenden Zusammenarbeit mit Polizeikräften gedrängt. Die Polizeikräfte in peripheren Gesellschaften seien häufig paramilitärischer Natur und in der Regel ,a far more effective and immediately useful counter-subversive instrument than the military.“, Komer Memo, 4.5.61, ,Staff Memoranda, Robert W. Komer, 4/17/61-5/15/61', NSF, M\&M, Box 321; als im Januar 1962 erneut Kürzungen der Polizeiprogramme (die Ausgaben fielen von \$14 Millionen im Finanzjahr 1958 auf \$11 in 1962) drohten, nahm Komer erneut den Kampf auf und setzte sich auch gegen eine Übernahme der Programme durch CIA oder das Department of Defense zur Wehr: ,In every country the police are almost invariably the first line of defense against subversion, demonstrations, riots, and even local insurrection.“; im Folgemonat plädierte er für die Einrichtung einer quasi-unabhängigen Organisation innerhalb AIDs, dem späteren Office of Public Safety, gegründet im November 1962. Siehe Komer Memo, 31.1.62, ,Staff [...] 1/62‘; Komer Memo, 7.2.62, ,Staff [...] 2/62‘, NSF, M\&M, Box 322, JFKL; vgl. auch Saunders Memo, 5.7.62; Taylor Memo, 7.12.62; Forrestal Memo, 10.12.62, FRUS 61-63, VII-VIII-IX Suppl., Doks. 411, 422, 423.
} 
umfassende Reorganisation und Modernisierung der iranischen Polizei federführend begleitet, u.a. mit der Aufstellung einer modernen ,riot control unit“ in Teheran selbst, einschließlich Mannschaftswagen und Wasserwerfern zur Kontrolle von Demonstrationen. Das Sicherheitsproblem in Iran sei ,primarily urban“, so eine lapidare Einschätzung in einer internen Analyse. ${ }^{212}$ Offenbar hatte während des Staatsbesuchs Mohammad Rezās im April 1962 auch eine Unterhaltung mit dem Präsidenten über einen weiteren Ausbau der Sicherheitskräfte mit amerikanischer Unterstützung stattgefunden. Einige Monate später gelang es dem Schah, auf Grundlage eines angeblich gegebenen Versprechens Kennedys materielle Unterstützung für den Aufbau einer 2.000 Mann starken Kommandobrigade für die verbesserte Kontrolle der Hauptstadt zu erlangen. ${ }^{213}$ Komer im NSC kämpfte unterdessen für die Umsetzung eines großzügigen Zivilhilfe-Programms in Iran. ${ }^{214}$

Die zunehmende Euphorie innerhalb der Kennedy-Administration für eine verstärkte Zusammenarbeit mit den iranischen Sicherheitsorganen im Kontext der entstehenden Counterinsurgency-Doktrin kontrastiert augenfällig mit dem ab dem Herbst 1961 erlahmenden Impetus zugunsten der gleichermaßen auf sozio-ökonomische Transformation und politische Liberalisierung gerichteten reformistischen Intervention. Die USA bleiben weiterhin als interventionistische Macht in Iran aktiv, doch kehrte die Zielsetzung der permanenten Intervention im Kern zurück zu den Leitprinzipien der Eisenhower-Administration: Innere Stabilität unter monarchischer Führung bei gleichzeitiger Beibehaltung der pro-westlichen außenpolitischen Orientierung. Zentral für das Verständnis des amerikanischen Politikwandels ist die wachsende Überzeugung, die Opposition in Iran in Schach halten zu können, und zwar nicht mittels Integration infolge der Umsetzung ihrer zentralen Forderungen hinsichtlich ökonomischer und politischer Reform, sondern durch Stärkung und Professionalisierung der iranischen Sicherheitskräfte. Ohne Frage spielte diese Wahrnehmung der tatsächlichen Stärke der iranischen Oppositionsgruppen eine Rolle in dieser Neueinschätzung.

Im NIE vom Februar 1961 hatte man noch apodiktisch festgestellt, dass ,,[...] profound political and social change in one form or another is virtually inevitable.“215 Angesichts der gegebenen Konstellation sei zudem revolutionärer Wandel die wahrscheinlichste Variante. Bemerkenswert ist der Wandel der Einschätzung im Juli 1962. Zwar konstatierte man auch im Sommer 1962 eine fortschreitende Schwächung der Monarchie als einigendem Faktor in Iran

\footnotetext{
${ }^{212}$ Vgl. Memo for Taylor, 17.4.62, vorherig. Anm.

${ }^{213}$ Vgl. oben S. 306, Anm. 43; Tehran \#3, 1.7.62; offenbar konnte sich Kennedy an keine Zusage von seiner Seite erinnern und vermutlich hatte es außer einer allgemeinen Unterhaltung über Sicherheitsfragen keine solche gegeben. Dennoch bewilligte die Administration \$500.000 für ein Iran Special Police Program und für die Aufstellung einer weiteren Spezialeinheit. Vgl. Johnson Memo, 25.7.62; Komer Memo, 26.7.62; Draft ICA Telegram, 25.7.62, ,Iran General 7/24/62-7/31/62‘, NSF, Countries, Box 116; Komer Memo, 7.7.62, ,Staff [...] Komer 7/62', NSF, M\&M, Box 322, JFKL.

${ }^{214}$ Vgl. Saunders Memo, 10.7.62, ,Counterinsurgency, Civic [...] Folder 2 of 2‘, NSF, RWK, Box 412; Saunders Memo, 11.7.62, ,Iran, 1961-1962 [Folder 2 of 2]', NSF, RWK, Box 424, JFKL; Coffin Letter, 15.12.62, ,Iran 1962 000.1---092', OASD/ISA Files: General and Country, 1962, Box 89, RG 330, NACP.

${ }^{215}$ CIA, NIE 34-61, 28.2.61, FRUS 61-63, XVII, S. 37.
} 
und in der Folge ein Anwachsen der ,long-range chances of a violent upheaval.“ Gleichzeitig aber gab man sich überzeugt, die Sicherheitsorgane seien in der Lage ,[...] of controlling any foreseeable disturbances short of a country-wide armed uprising." Ein solcher Aufstand aber sei angesichts des Zustands der Opposition so gut wie auszuschließen. Die nationalistische Opposition sei klein, schwach organisiert und ineffizient. Die große Masse der Landbevölkerung sei zudem politisch apathisch und ohne politische Führung. Gefahr von den weitgehend ohnmächtigen Stämmen drohe nur bei gleichzeitigen Unruhen in den urbanen Zentren. Das Fazit lautete: ,[...] active insurgency is unlikely in Iran in the near future.“216

Im Kontext der Beratungen über Iran im Rahmen der Special Group (CI) erfolgte denn auch die formale Abkehr von den Prinzipien des Task Force Report vom Mai 1961. Die oben analysierten Guidelines for Policy and Operations vom September 1962 hatten bereits den Widerstand des State Department, nunmehr geduldet vom Weißen Haus, gegen eine Fortsetzung der reformistischen Politik gegenüber Iran dokumentiert. Gemäß der bereits diskutierten OIDP vom August 1962 und infolge der Aufnahme Irans in die Liste der „kritischen Staaten“ im Juni wurde auch für Iran ein Country Internal Defense Plan (CIDP) formuliert, der die operative Umsetzung der unterschiedlichen Ideen und Konzepte der Aufstandsbekämpfung und inneren Sicherheit enthielt. Der CIDP für Iran, datiert vom 30. Oktober 1962 und erstmals deklassifiziert für die vorliegende Studie, wurde Anfang November von der Special Group (CI) diskutiert und verabschiedet. Obgleich mit der grundsätzlichen Ausrichtung des CIDP weitgehend zufrieden, leistete das State Department und insbesondere NEA Widerstand gegen die Behandlung der Strategie gegenüber Iran im Rahmen der Special Group (CI). Dabei ging es in erster Linie um die Verteidigung der eigenen Kompetenzen und Missfallen gegen das Eindringen des Weißen Hauses und anderer Ressorts in ureigene Kernbereiche des Außenpolitik. Der Versuch, Iran von der „Critical List“ der Special Group (CI) streichen zu lassen, scheiterte jedoch am Widerstand der anderen Behörden. ${ }^{217}$ Dennoch betonten Holmes und Bowling in der entscheidenden Sitzung der Special Group (CI), es gäbe in Iran keine klar definierbare Bedrohung mehr, auf die man die neuen CounterinsurgencyKonzepte anwenden könne. Sie betonten zudem den sich zu dieser Zeit abzeichnenden neuen

\footnotetext{
${ }^{216}$ CIA Memo, ,Insurgency in Iran‘ 25.7 .62 , ,Iran, 1961-1962 (Folder 1 of 2)`, NSF, RWK, Box 424, JFKL; eine Einschätzung der Lage in Iran durch die CIA im August 1962 enthielt einen Annex über innere Sicherheit, der die verschiedenen Sicherheitsorgane eingehend analysierte. Insbesondere der 2.000 bis 2.400 Mann starken SAWAK wurde Effizienz bescheinigt, der nationalen Polizei Durchschlagskraft in der Hauptstadt, allerdings weniger in den Provinzen. Vgl. Annex ,Internal Security in Iran', encl. to CIA Board of National Estimates Memo, 16.8.62, encl. to Komer Memo, 18.8.62, ,Iran, 1961-1962 (Folder 1 of 2), NSF, RWK, Box 424, JFKL; vgl. FRUS 61-63, XVIII, S. $87 n 3$.

${ }^{217}$ NEA hatte im September vergeblich die Entfernung Irans von der Liste angemahnt. Man bezog sich auf das jüngste SNIE vom September und die darin enthaltene Feststellung, es gebe derzeit keine Oppositionsgruppe in Iran, die glaubhaft in der Lage wäre, das Schah-Regime zu stürzen. DCI McCone kritisierte nach der Erörterung des ersten Progress Report für den IRAN-CIDP im März 1963, dass dieser „,covert action and political action“ enthalte, was formal eigentlich die Domäne der CIA und Teil der Entscheidungsbefugnis der Special Group (5412 Committee) sei. Ein Memo von Alexis Johnson, das den nicht freigegebenen Progress Report paraphrasiert, lässt als Hintergrund eine verstärkte Zusammenarbeit mit dem SAWAK vermuten. Vgl. Talbot Memo, 19.9.62, ,SPECIAL [...] 10/31/62‘, Lot 68 D 451, SG (CI) Records, Box 1; Johnson Memo, 27.2.63, ,SPECIAL [....]3/7/63', ebd., Box 2; Lee Memo, 21.9.62; Lee Memo, 20.12.62; Grant Memo, 5.11.62, ,3-A Counterinsurgency, 1962‘, NEA, GTI: RIAD, 1958-63, Box 6; vgl. FRUS 61-63, XVIII, S. $373 n 1$.
} 
Reformwillen Mohammad Rezās, der nun selbst die Landreform - „truly revolutionary“ laut dem Botschafter - für sich und seine Ziele entdeckt habe. ${ }^{218}$ Angesichts dieser durchsichtigen bürokratischen Strategie, mokierte sich Bob Komer später über die Behauptung der State Department-Vertreter, dass Iran das „,stabilste Land im Nahen Osten sei“ und titulierte Holmes” und Bowlings Behauptungen über die interne Stabilität in Iran als „one of the better snow jobs of recent memory“. ${ }^{219}$

Wenn auch für den zukünftigen Verlauf der amerikanischen Politik gegenüber Iran von Bedeutung, so interessiert an dieser Stelle weniger der anhaltende Kampf des State Department um möglichst unbeeinträchtigte Kontrolle der Iranpolitik, sondern vielmehr der Einfluss der nun konkretisierten Counterinsurgency-Konzeptionen auf eben diese. In dieser Hinsicht ist der CIDP für Iran in der Tat ein Schlüsseldokument. Der CIDP war weit mehr als eine Bestandsaufnahme der inneren Sicherheit und der verschiedenen Sicherheitsorgane in Iran, wie man vielleicht von einem Bericht im Rahmen der OIDP und im Kontext von „Aufstandsbekämpfung“ hätte erwarten können. Zwar behandelte der CIDP diese Fragen im Detail, doch befasste er sich zugleich mit der amerikanischen Politik gegenüber Iran im Ganzen, also auch politische, wirtschaftliche, militärische und andere Themenkomplexe miteinbeziehend. In gewisser Weise war die Breite der CIDP der versuchten Übernahme einer allgemeinen Steuerungsfunktion für die US-Strategie entlang der Peripherie durch die Special Group (CI) geschuldet. De facto löste der CIDP - gemeinsam mit den bereits erwähnten Guidelines - die Task Force-Politik vom Mai 1961 ab. ${ }^{220}$ In ihm wurden die nun wirkungsmächtigsten Komponenten der amerikanischen Iranpolitik zusammengefasst: die weitgehende Aufgabe des reformistischen Interventionismus und die weitere Militarisierung und „Versicherheitlichung“ der Beziehungen.

Zentral für den CIDP - auch für die von Holmes und Bowling in der Special Group (CI) vertretenen Linie - war die Einschätzung de Bedrohungslage in Iran: „At present, there is no clearly identifiable threat to internal stability. “221 Im Task Force Report hatte es noch ausdrücklich geheißen: „The continuing trend toward revolution and chaos in Iran has reached the point where the U.S. must take vigorous action.“222 Dieser Alarmismus war nun einer eher nüchternen Analyse gewichen, deren Kernaussage war, dass bei entsprechender Unterstützung

\footnotetext{
${ }^{218}$ Meeting of the Special Group (CI), 5.11.62, FRUS 61-63, XVIII, S. 201f.; Bowling beschrieb bündig die Kehrtwende des Schahs: ,Whereas in the past the Shah was allied and supported by the Army and the elite versus the middle class and students, he has now through his reform programs allied himself with the Army, the peasantry and the urban proletariat versus the traditional elite, students and middle class."

${ }_{219}$ Komer Memo, 5.11.62, ,Iran, 1961-1962, White House Memoranda', NSF, RWK, Box 424, JFKL; Komer wandte sich mit Nachdruck gegen die sich abzeichnende Position NEAs zugunsten einer Anpassung der eigenen Entwicklungsziele an die des Monarchen: „But if moving at the Shah's pace means just bottling up revolution, we're again postponing the inevitable. We need a 'controlled revolution' in Iran [...]."

${ }^{220}$ Formal gibt es allerdings zwischen Task Force-Bericht und der CIDP eine direkte Bezugnahme, aber nicht zwischen letzterem und den Guidelines. Formal wurde der Task Force Report erst im April 1963 durch die Antwort des State Department auf NSAM N²28 ad acta gelegt. Siehe unten Kapitel V.3.

${ }^{221}$ Report ,Outline of Country Internal Defense Plan-Iran' [im Folgenden: CIDP], 30.10.62, encl. to Maechling Memo, 1.11.62, ,Iran, 1961-1962 [Folder 1 of 2] ', NSF, RWK, Box 424, JFKL, S.1.

${ }^{222}$ Iran Task Force Report, Recommendations, S. 2.
} 
für die iranischen Sicherheitsorgane der zuvor noch als fast unabänderlich eingeschätzte revolutionäre Wandel kontrolliert bzw. unterdrückt werden könne. Bezüglich der politischen Verfasstheit Irans kehrte der CIDP unumwunden zur alten Eisenhower-Position zurück: Unterstützung der Monarchie als Garant für Stabilität und pro-westliche Orientierung. Die nationalistische Opposition, zugleich Vertreter der zuvor als Träger einer neuen politischen Synthese identifizierten urbanen Mittelschichten, war nunmehr herabgestuft zu einem reinen (und bewältigbaren) Sicherheitsproblem. ${ }^{223}$ Der Schwerpunkt wurde auf eine Stärkung der Sicherheitsorgane gesetzt, insbesondere die Schaffung von Polizeikräften, zuvorderst in der Hauptstadt, aber zukünftig auch in den Provinzmetropolen, mit einer ,,riot control capability which will obviate the necessity for the use of military units to quell demonstrations or riots. “224 Zugleich sollten sowohl Militär wie auch die paramilitärische Gendarmerie in ihren Kapazitäten zur Aufstandsbekämpfung gestärkt werden, sowie die Kooperation mit dem SAWAK und anderen geheimdienstlichen Stellen intensiviert werden. ${ }^{225}$ „Psychologische“ Maßnahmen gegenüber der iranischen Bevölkerung sollten unter anderem, ,[...] the need for disciplined order as a prerequisite to modernization, economic stability and personal freedom" betonen. Der CIDP listete zudem detailliert die laufenden sozio-ökonomischen Entwicklungs- und Modernisierungsvorhaben und den jeweiligen amerikanischen Beitrag auf, verzichtete aber auf jede Erwähnung politischer Transformation. Stattdessen wurde konstatiert: „It must be recognized, however, that within the framework of acknowledging Iran's sovereignty and independence the degree to which external influence or assistance can be applied effectively is severely limited.“226

Die Verabschiedung des CIDP kann als vorläufiger Höhepunkt der Anwendung der Counterinsurgency-Konzepte auf den iranischen Fall angesehen werden. Es ist wohl kein Zufall, dass der Abschluss dieses Trends hin zu einer konterrevolutionären Einstellung gemäß der OIDP vom August 1962, der zugleich eine Rückbesinnung auf militärische und polizeiliche

\footnotetext{
${ }^{223}$ Bezeichnenderweise war die Opposition nunmehr in der Diskussion der ,vulnerabilities“ im CIDP nicht mehr unter ,political“" sondern unter ,security“" gelistet. Vgl. CIDP, S. 2.

${ }^{224}$ Ebd., S. 4.

225 Die etwa 28.000 Mann starke Gendarmerie hatte bereits im Rahmen des MAP ein landesweites Kommunikationssystem erhalten, das ihre Effizienz enorm steigerte. Beschlossen war zudem die Lieferung von 16 Cessna-Leichtflugzeugen und fünf Helikoptern sowie 500 Lastwagen, um die Mobilität der Gendarmerie zu erhöhen. Allein im Finanzjahr 1963 wurden zudem 58 Offiziere in den USA ausgebildet, zusätzlich zu den seit 1952 trainierten 392 Gendarmen. Bezüglich der iranischen Polizei strebte man die Umformung von einem traditionellen Überwachungsdienst hin zu einer ,preventive law enforcement agency adequate for a changing society" an. Konkret bedeutete dies Unterstützung und Beratung durch die acht amerikanischen (CIA-)Berater in der Public Safety Division in Teheran und eine umfassende Ausrüstung der Polizei mit allen denkbaren Mitteln zur Kontrolle von Demonstrationen und urbanen Unruhen. 16 Polizeioffiziere sollten 1963 in den USA weitergebildet werden (121 seit 1952). Zudem hatte man nun alle Hemmungen zur Zusammenarbeit mit der zweifelhaften National Resistance Organization, einer ultranationalistischen paramilitärischen Miliz, abgelegt, die innerhalb von fünf Jahren auf eine Sollstärke von 50.000 Mann ausgebaut werden sollte, offenkundig als Kampftruppe gegen innere Aufstände konzipiert. Ein Pentagon-Dokument sprach von einem integrierten Plan ,[...] which will include coordination of the Special Forces and any indigenous paramilitary forces such as the Gendarmerie." Bei der Ausbildung der iranischen Armee machte sich eine Schwerpunktsetzung auf Konzepte der Aufstandsbekämpfung bemerkbar. Zu den seit 1950 in den USA ausgebildeten 3721 Offizieren kamen 1963489 weitere hinzu. ARMISH/MAAG hatte zu dieser Zeit 517 Berater in Iran. Vgl. ebd., S. 10f., 13; Bowen [US European Command] Report, 16.9.61, ,Iran 1962 000.1---092‘, OASD/ISA Files: General and Country, 1962, Box 89, RG 330, NACP.

${ }^{226}$ CIDP, S. 13.
} 
Kontrolle der Opposition und eine Abkehr von ambitionierten Projekten politischer wie sozioökonomischer Transformation bedeutete, zeitlich mit der bereits analysierten grundsätzlichen Beschwerde Bob Komers und anderer überzeugter reformistischer Interventionisten zusammenfiel. Zwar unterstützte auch Komer enthusiastisch die Reorientierung der Militärhilfe auf Aufstandsbekämpfung und ,internal defense“. Zugleich aber glaubten er und sein Mitstreiter an die Notwendigkeit einer umfassenden Transformation Irans, angeregt durch eine aktivistische und interventionistische Politik der amerikanischen Führungsmacht. Die verstärkte Repression und Kontrolle im Innern könne in einer Übergangsphase hilfreich sein, mittelfristig aber sei sie kein Ersatz für eine umfassende und vor allem politische Neuordnung der internen Machtstrukturen. Angesichts des generellen Wandels der Grand Strategy der Kennedy-Administration gegenüber den Staaten der Peripherie fanden diese kritischen Stimmen aber kein Gehör. Insgesamt entwickelte sich die Iranpolitik parallel zur allgemeinen Strategie, von einer Betonung interner Reform in der ersten Jahreshälfte hin zur einer graduell wahrnehmbaren, zunehmend einseitigen Betonung innerer Sicherheit zum Jahreswechsel $1961 / 62$.

Nach außen aber drang diese Umorientierung der Kennedy'schen Strategie nur begrenzt. Die berühmte Rede des Präsidenten zum 100-jährigen Jubiläum der University of Washington in Seattle am 16. November 1961 enthielt allerdings einige (häufig zitierte) Passagen, die eine Abkehr von der zu Beginn der Administration angestrebten umfassenden Transformationspolitik nahe legten:

In short, we must face problems which do not lend themselves to easy or quick or permanent solutions. And we must face the fact that the United States is neither omnipotent or omniscient-that we are only 6 percent of the world's population-that we cannot impose our will upon the other 94 percent of mankind-that we cannot right every wrong or reverse each adversity-and that therefore there cannot be an American solution to every world problem. ${ }^{227}$

In der Kennedy-Forschung wird die Seattle-Rede meist auf die Grundsatzentscheidung bezüglich Vietnam - die historiographische Debatte dreht sich in der Regel um die tatsächliche Tragweite der Entscheidung - vom Vortag bezogen. Kennedy verweigerte dabei eine massive amerikanische Militärintervention in Südostasien - eine Woche später autorisierte er allerdings eine stark intensivierte Anstrengung in Südvietnam auf Basis der fast zeitgleich ausformulierten und nun vorliegenden ersten Konzepte zur „Aufstandsbekämpfung“. Genau in diese Zeit fällt zudem die bereits erwähnte Entscheidung zu Personalverschiebungen in Weißem Haus und State Department, unter anderem die Entfernung von sowohl Bowles wie auch Rostows aus dem engsten Beraterkreis. ${ }^{228}$ Diese waren Widersacher bezüglich Südostasien - mit Rostow 
als größtem Scharfmacher innerhalb der Administration -, teilten andererseits aber ambitionierte Vorstellungen über die Möglichkeiten reformistischer Transformation nach amerikanischem Vorbild, gerichtet auf die neuen Staaten und Gesellschaften. Der in Kennedys Seattle-Rede zum Ausdruck gekommene ,foreign policy minimalism“, die Einsicht in die begrenzten Einflussmöglichkeiten auf andere Gesellschaften trotz Supermacht-Status, scheint in der Tat eher zum Zurückfahren der Transformationsprogramme gegenüber Lateinamerika, Iran und anderen Staaten zu passen als zur Frage des Ausmaßes amerikanischer Involvierung in Vietnam. ${ }^{229}$ Die Entstehungsgeschichte der Seattle-Rede ist anhand der Aktenlage allerdings nicht einfach nachzuzeichnen. Eine Reihe von Einflüssen auf die Rede- neben den Vorgaben Kennedys selbst - ist belegbar. Neben Arthur Schlesinger Jr. und Theodore Sorensen gab es zudem Eingaben und Ideen von Dritten, z.B. dem Stab eines der Senatoren aus dem Bundesstaat Washington, die in die letztendliche Fassung der Rede integriert wurden. ${ }^{230}$ Graduell wurden die zwei Hauptbotschaften der Rede herausgearbeitet, erstens die hier nur bedingt interessierende Dichotomie zwischen übertriebener Belligerenz und Kompromissbereitschaft angesichts der globalen Herausforderungen - sinnbildlich für die Positionen Rostows und Bowles` bezüglich Vietnam - sowie zweitens die Betonung des Widerspruchs zwischen eigenen Idealen (,We must work with certain countries lacking in freedom in order to strengthen the cause of freedom.") und außenpolitischen Notwendigkeiten, inklusive der Einsicht in die Begrenzungen der eigenen Macht. Die oben zitierte Passage findet sich, in leicht abgewandelter Form, bereits in den ersten Entwürfen der Rede, also noch vor dem NSC-Meeting zu Vietnam. Allerdings gingen dem NSC-Meeting am 15. November mehrtägige Beratungen über den einzuschlagenden Kurs voraus, sodass ein Bezug zur Vietnam-Entscheidung letztlich dennoch nicht auszuschließen ist. ${ }^{231}$ Für die vorliegende Untersuchung aber ist zu konstatieren, dass Präsident Kennedys Seattle-Rede grundsätzliche Elemente enthielt, die im Zusammenhang mit

22.11.61, ebd., S. 656f.; zu den Umbesetzungen und Rostows Entfernung aus dem Weißen Haus vgl. Bundy Memo, 15.11.61, ebd., S. 612ff.; ed. note in ebd., S. 670f.; vgl. DALleK, Kennedy, S. 455.

${ }_{229}$ „Foreign Policy Minimalism“ geprägt von j(J)anet Lang. Vgl. die anregenden Debatten über die Abfolge der Entscheidungen und den historischen Stellenwert der Seattle-Rede in BLIGHT/LANG/Welch, If Kennedy Had Lived, S. 53-95.

${ }^{230}$ Enge Berater des Senators von Washington State und geistigen Vaters des heutigen Neokonservatismus, Henry «Scoop» Jackson, Dorothy Fosdick und Robert Tufts, hatten einen eigenen Redevorschlag geschickt. Darin enthalten waren bereits spätere zentrale Bestandteile der Seattle-Rede, insbesondere die Notwendigkeit der Gelassenheit und Geduld in der langen Systemkonkurrenz mit der Sowjetunion. Man müsse alternative Entwicklungswege in der Dritten Welt tolerieren, solange diese nicht mit Unterwerfung gegenüber dem Kommunismus einhergingen. Allerdings verfüge man hinsichtlich der Freien Welt über große Gestaltungsmöglichkeiten. Vgl. Fosdick-Tufts Draft, o.D., ,Address [...] Seattle, Washington, 16 November 1961', POF, Speech Files, Box 036, JFKL.

${ }^{231}$ Schlesinger Jr. sandte dem Präsidenten und Sorensen sowie Goodwin am 15. November, also einen Tag vor der Rede, einen Entwurf ,based on themes thrown out the other day by the President for possible inclusion in the speech."; offenbar lag das Gespräch mit Kennedy ein bis zwei Tage zurück. In dieser Fassung ist die oben zitierte Passage bereits enthalten, handschriftlich ergänzt durch die Feststellung, dass man nur 6\% der Weltbevölkerung stelle. Vgl. Draft for Seattle, attached to Schlesinger Memo, 15.11.61; Sorensen Draft o.D., ,Address [...] Seattle, Washington, 16 November 1961', POF, Speech Files, Box 036; Schlesinger Memo, 15.11.61, ,Address [...] Seattle, 16 November 1961', Schlesinger Papers, Speech File, 1961-1964, Ser., Remarks for the President, 19611963, Subs., Box WH67; die Version in den POF ist an einer Stelle handschriftlich kommentiert. Die früheste Version der Rede ist offenbar eine handschriftliche Version im Sorensen-Nachlass, die bereits die Passage mit „omnipotent“ enthält, wenn auch an einer anderen Stelle der Rede. Eine abgetippte Version, bezeichnet als Draft \#1 ist datiert auf den 14.11. Im Folder befindet sich auch der so bezeichnete Draft \#2, datiert auf den 15.11. Vgl. Sorensen Draft, o.D., Sorensen Draft \#1, 14.11.61, Sorensen Draft \#2, 15.11.61, ,Western [...] Washington anniversary, 16 November 1961‘, Sorensen Papers, JFK Speech Files, 1961-1963, Ser., Box 65, JFKL. 
dem Wandel der Gesamtstrategie im Global Cold War zu sehen sind. Die Aufgabe des reformistisch-interventionistischen Projekts in Iran steht mit großer Wahrscheinlichkeit in direktem Bezug zu diesem Wandel. Beginnend im Herbst 1961, spätestens zum Jahreswechsel 1961/62, lässt sich die Priorisierung von Stabilität und innerer Sicherheit gegenüber den ambitionierten Transformationsideen der frühen Phase belegen. Augenfällig ist die Hintanstellung dieser Ideen im Kontext des Schahbesuchs im April 1962 und dem Scheitern der Amini-Regierung. Zum Zeitpunkt des letzten vehementen Protests der Komer-HansenGruppe im Herbst 1962 hat das reformistische Projekt bereits seit langem seine Dynamik eingebüßt. Zu eben dieser Zeit erfolgen denn auch die ersten formalen Schritte zur Ablösung der Task Force-Politik, im Grunde die vorhergehende politische Aufgabe des Projekts nunmehr nur nachvollziehend. Im Laufe des Jahres 1963 erfolgte dann die Aufgabe der Restbestände dessen, was einmal ein umfassendes politisches, soziales wie ökonomisches Transformationsprojekt in Iran darstellte.

Die hier ausformulierte These zum Wandel der Politik der Kennedy-Administration gegenüber den Staaten der Peripherie widerspricht im Kern der weitgehend akzeptierten These einer in erster Linie ideologisch geprägten und zumeist unkontroversen Verfolgung transformativer Konzepte durch die New Frontier. Das Gros der bestehenden Literatur registriert zwar die vertiefte Aufmerksamkeit für Sicherheitsfragen und Aufstandsbekämpfung, sieht diese aber in der Regel als militante Ergänzung zur vorwiegend sozio-ökonomisch ausgerichteten Modernisierungspolitik. Eben diesen militärischen und sicherheitspolitischen Trend als de facto-Aufgabe der ambitionierten Transformationsziele zu interpretieren und als Auslöser einer umfassenden Politikwende zu analysieren, ist demzufolge als origineller Beitrag für die Kennedy-Forschung im Allgemeinen und das Feld der amerikanisch-iranischen Beziehungen im Besonderen anzusehen. ${ }^{232}$

\section{3. «Weiße Revolution» und Aufgabe des reformistischen Interventionismus}

Mit dem Scheitern Komers im Oktober 1962, eine neue „Iran exercise“ zu initiieren, eröffnete sich für Schah Mohammad Rezā die Möglichkeit, den immer sichtbarerer werdenden Schwenk in der US-amerikanischen Iranpolitik in eine ihm genehme Richtung zu lenken. Der Widerstand der Komer-Hansen-Gruppe gegen die einseitige Unterstützung des Schahs bestand jedoch weiter, wenn auch seit Mitte 1962, da ohne spürbare Unterstützung durch Präsident Kennedy, zunehmend kraftloser. Die Priorisierung der internen Stabilität bei gleichzeitiger Unterstützung des Monarchen kritisierte Komer weiterhin scharf:

${ }^{232}$ Ein verwandtes Argument, wenngleich ohne Identifizierung des Zeitpunkts und des Wandels der Grand Strategy bei STREETER, Eternal Counter-Insurgency; Streeter sieht die militärischen Komponenten allerdings als Absicherung der Transformationsprogramme, nicht als deren Ersatz. 
But if moving at the Shah's pace means just bottling up revolution, we're again postponing the inevitable. We need a 'controlled revolution' in Iran, and [...] we must promote one. We don't know where we're going in Iran, and are inviting greater risks by refusing to take lesser ones. ${ }^{233}$

Tatsächlich war der Schah offenbar zu dem Schluss gekommen, dass weiterer USamerikanischer Druck zugunsten umgreifender Reformen nur zu verhindern sei, indem er nun selbst aktiv als Reformer tätig werde. Auf der rhetorischen Ebene hatte sich der Schah bereits seit den 1940er Jahren als Reformer stilisiert, konkrete Umsetzungen waren aber bis zur Gegenwart immer bruchstückhaft geblieben und die wenigen Schritte waren selten ambitioniert. Tatsächlich waren das Ausbleiben „echter“ Reformen und das Verharren der iranischen Gesellschaftsordnung in ihrer althergebrachten Form - insbesondere die fortgesetzte Besetzung der meisten Schlüsselpositionen mit Angehörigen der traditionell einflussreichen Familien - schon immer ein Hauptkritikpunkt der linken und auch der nationalistischen Opposition gewesen. Das Anwachsen professioneller Mittelschichten und die Ausweitung höherer Bildung machten stärkeren gesellschaftlichen Druck zugunsten der Einleitung umfassender Reformen für die Zukunft absehbar. Persönlich war Mohammad Rezā schon immer Reformvorstellungen, auch radikaleren, zugeneigt gewesen. Ihn hielt in erster Linie die Sorge zurück, infolge der mit einer weiteren Modernisierung einhergehenden Umwälzungen in Konflikt mit den traditionell einflussreichen Schichten, insbesondere den Landbesitzern, aber auch der schiitischen Geistlichkeit, zu geraten, die zudem, anders als die auf Reformen drängenden Mittelschichten, weitgehend loyale gesellschaftliche Träger der iranischen Monarchie in Iran waren. ${ }^{234}$ Andererseits eröffnete die Annahme einer reformistischen Leitrolle die Möglichkeit, der Pahlawi-Dynastie eine neue ideologische Legitimationsbasis als Trägerin nationalistischer Zukunftsvorstellungen einer starken, unabhängigen und technologisch mit dem Westen gleichrangigen Nation zu verleihen. Ende 1962 entschied sich Mohammad Rezā für diese Variante und die „Revolution des Schahs und des Volkes“, besser bekannt als „Weiße Revolution“ (engelāb-e sefid), wurde nun mit Nachdruck initiiert. Das Konzept einer solchen „Revolution von Oben“ geisterte offenbar schon länger durch die Korridore der Macht, wurde aber aufgrund der bisherigen Zurückhaltung Mohammad Rezās nie ernsthaft in Erwägung gezogen. Eine Schlüsselperson in diesem Kontext war wieder einmal sein Intimus Asadollah 'Alam, der bereits im August 1958 die Idee einer Weißen Revolution gegenüber britischen Diplomaten ausgeführt hatte:

To the same end [...] a progressive monarchy under a young ruler more popular than Colonel Nasser, Asadullah hoped to prevail upon the Shah to be rid of the present 'establishment' the existing ruling classes must give place to new and younger men. The old gang were not of course to be hurt; this was a white not a red revolution; but the Shah must sack them all. ${ }^{235}$

\footnotetext{
${ }^{233}$ Komer Memo, 5.11.62, ,Iran, 1961-1962, White House Memoranda‘, NSF, RWK, Box 424, JFKL.

${ }^{234} \mathrm{Vgl}$. ANSARI, Myth, S. 3f.

${ }^{235}$ Kellas Minute, 12.8.58, FO 371/133006, TNA:PRO; 'Alam erwähnte dabei, dass es ihm bisher nicht gelungen sei, den Schah von den Vorzügen seiner „Weißen Revolution“ zu überzeugen. Im Kern stand auch für "Alam bereits 1958 eine Landreform als zentrale Maßnahme. Ansonsten hielt er vor allem die psychologischen Effekte
} 
Die Iranforschung debattierte von Anbeginn an, wie diese Einleitung einer „Revolution von Oben“ zu bewerten sei. Vor der Revolution dominierten - wenig überraschend - die eher positiven Bewertungen der Weißen Revolution, die eine entscheidende Rolle in der nun einsetzenden rapiden Modernisierung und Transformation Irans nach westlichem Vorbild und dem Erlangen eines herausgehobenen Status in der internationalen Politik zu spielen schien. Im Nachgang der Revolution dagegen dominierten Stimmen, die der Weißen Revolution eher negative Gesamtwirkungen zuschrieben bzw. sie sogar als eine der Grundursachen des späteren Untergangs der Pahlawi-Monarchie interpretierten. In der Retrospektive ist sich die Forschung weitestgehend einig, dass die große Mehrheit der im Rahmen der „Weißen Revolution“ umgesetzten Reformschritte keine nennenswerten Effekte zeitigten. Die nicht zu bestreitende umgreifende Transformation der iranischen Gesellschaft während der 1960er Jahre ist in erster Linie auf den ohnehin stattfindenden und früher einsetzenden sozialen Wandel und insbesondere auf das rasante Wirtschaftswachstum zurückzuführen, wobei die Weiße Revolution auf Letzteres kaum positiven Effekt gehabt haben dürfte. Von dieser Einschätzung auszunehmen ist allerdings das zentrale Reformvorhaben: die Landreform. Zwar ließ auch hier der frühe Enthusiasmus bald spürbar nach, dennoch ist nicht zu bestreiten, dass die Landreform umgreifenden Wandel in den ländlichen Gebieten und bei den Besitzverhältnissen auslöste. Bis 1971 wurden nach verbreiteten Schätzungen etwa 40-50\% der Gesamtfläche neu verteilt somit im Effekt weitaus eingreifender als z.B. die Landreform im revolutionären Ägypten, die nur etwa $12 \%$ der agrarischen Gesamtfläche umfasste. ${ }^{236}$

Sowohl die Landreform als auch die übrigen Bestandteile der Weißen Revolution hatten sich schleichend als einigermaßen kohärentes sozio-ökonomisches Reformprogramm bereits seit etwa 1960 etabliert, ohne dass die Implementierung mit großem Nachdruck verfolgt worden wäre. Bereits ab November 1961 unter Amini waren die ersten sechs Reformpunkte - inklusive die zentrale Landreform - ausformuliert worden. Die ursprüngliche Gesetzgebung zur Landreform erfolgte bereits im Januar 1962. Entscheidender Auslöser für die nun einsetzende Dynamik auf diesem Feld war eine Erweiterung der bestehenden Gesetzgebung zur Umsetzung einer Landreform vom 17. Januar 1963, wodurch diese auch auf die Ländereien der etwa 200.000 mittleren und kleineren Landbesitzer ausgedehnt und somit eine grundlegende

\footnotetext{
für ausschlaggebend - der Schah müsse sich in einen populistisch-nationalistischen Reformer verwandeln, um endlich breite Unterstützung in der iranischen Bevölkerung zu erlangen. Ein britischer Offizieller kommentiert: „But it would remain to be seen whether a revolution (red, white or blue) can be fathered upon a not very popular monarch [...]."; vgl. auch ANSARI, Myth, S. 5 f.

${ }_{236}$ Vgl. LAMBTON, Land Reform, S. 216-40; NAJMABADI, Land Reform, S. 99-107; HoogLund, Land and Revolution, S. ix, 60-70, 138-52; Lambton ist allerdings eher den Pahlavists zuzuordnen. Ihre Bücher galten lange Zeit als Standardwerke, sind aber im Sinne britischer und amerikanischer Politik allzu wohlgesonnen gegenüber der Politik des Schahs. In einer Audienz mit Mohammad Rezā im Oktober 1961 sagte sie z.B. dem Monarchen zu „to make as favorable an article as possible“. Vgl. MemCon, Okt. 1961, ,Iran, Security 1961-1963“, POF, Countries, Box 119, JFKL; zu Lambtons Rolle im Coup von 1953 vgl. LouIS, Britain and the Overthrow, S. 1307, 163ff.; erschreckend servil DENMAN, King's Vista, S. 300; einseitig und autobiographisch geprägt ist MAJD, Resistance to the Shah; für eine interessante Langzeitstudie der Folgen der Landreform vgl. AJAMI, Peasant to Farmer, S. $331 \mathrm{ff}$.; vgl. FORAN, Fragile Resistance, S. 318-25.
} 
Transformation der Besitzverhältnisse eingeleitet wurde. ${ }^{237}$ Erst mit der Übernahme des Reformprogramms als monarchische Aufgabe durch Mohammad Rezā Ende 1962 erschien die Weiße Revolution in der öffentlichen Wahrnehmung als zentrales Politikziel, bekräftigt durch das anschließende nationale Referendum Anfang 1963. In der Folgezeit wurden die anfänglich sechs Reformschritte ausgeweitet auf zwölf und bis Ende der 1970er Jahre auf $17 .^{238}$ Letztlich gelang die Präsentation der Pahlawi-Monarchie als Triebkraft reformistischer Umgestaltung und Modernisierung Irans nach außen besser als nach innen. Zwar schien sich auch innerhalb der iranischen Bevölkerung in den Folgejahren die Wahrnehmung der anfangs eher zynisch betrachteten Reformschritte der Regierung zu verändern, wohl nicht zuletzt beeinflusst durch den augenfälligen Wandel im Land. Bedeutsamer waren im historischen Rückblick aber wohl die Akzeptanz des Schahs als großer Reformer durch westliche Öffentlichkeiten - übrigens durchaus auch auf Seiten der Regierungen des Ostblocks -, und die Veränderung des Images Mohammad Rezās. Tatsächlich kann die letztendliche Einleitung der Weißen Revolution nicht ohne Einbeziehen des komplexen Verhältnisses zum amerikanischen Patron und der Wechselwirkungen mit dem kurzlebigen reformistischen Interventionismus der KennedyAdministration verstanden werden. ${ }^{239}$

Botschafter Julius Holmes fasste Ende November 1962 die Neuerungen in den staatlichen Reformbemühungen zusammen. Anders als in vorherigen Initiativen habe nun der Schah selbst - nach der Rückgewinnung der Kontrolle über das Regierungshandeln - die Führung der Reformumsetzung übernommen. Als „revolutionary monarch“, so Holmes, habe der Schah die Überzeugung gewonnen, dass Iran so rasch wie möglich Anschluss an die westliche Moderne finden müsse. Zentral für das Reformprogramm des Schahs waren weiterhin die Landreform, die bereits vom Amini-Kabinett unter dem als radikal bekannten Landwirtschaftsminister Arsanğāni eingeleitet worden war, sowie die Bekämpfung der Korruption. Des Weiteren proklamierte der Schah nun Pläne für die Herstellung sozialer Gerechtigkeit, z.B. durch Gewinnbeteiligung der Arbeiterschaft, umfassende Verwaltungsreformen, Alphabetisierungskampagnen und die Einführung des Frauenwahlrechts. ${ }^{240}$

Zentral für die innere Machtbalance in Iran war aber die beschleunigte Umsetzung der

\footnotetext{
${ }^{237}$ Vgl. NAJMABADI, Land Reform, S. 92-7; LAMBTON, Land Reform, S. 60-86; die Gesetzgebung vom Januar 1962 war im Grundsatz nur eine Erweiterung des Gesetzes von 1960. Vgl. HooGLund, Land and Revolution, S. 53f.; zur entscheidenden Erweiterung der Landgesetzgebung vom 17. Januar 1963 vgl. ebd., S. 104ff.; angesichts des Ausmaßes der Umverteilung und der möglichen politischen Folgen wurde dieser Schritt allerdings sogleich wieder abgeschwächt. Vgl. Tehran \#A-518, 18.2.63, E 12 IRAN, CF 63.

${ }^{238}$ Vgl. Ansari, Myth, S. 12f.; Milani, The Shah, S. 292ff.; AfKhAmi, Life and Times, S. 208-33.

${ }^{239}$ Mohammad Rezā erwartete volle Unterstützung seitens der USA, ,now that it is clear that Iran is not a reactionary outmoded monarchy.“; CIA Information Report, 12.1.63, ,Iran General 1/63“, NSF, Countries, Box 116A, JFKL; Interessant die Interpretation Ramazanis, der die Weiße Revolution als in erster Linie durch Unabhängigkeitsbestrebungen induziert interpretiert. Amerikanischer Reformdruck auf der einen Seite und Entspannung mit der UdSSR auf der anderen Seite hätten den neuen Ansatz erst ermöglicht. Infolge der Détente mit dem nördlichen Nachbarn konnten Ressourcen nach innen umgelenkt werden - eine mit der Quellenlage allerdings nicht zu vereinbarende These. Vgl. RAMAZANI, Iran's White Revolution, S. 129ff.; vgl. auch SAIKAL, Rise and Fall, S. 79-92; eine interessante Analyse des parallel entstehenden ideologischen Gebäudes eines „Pahlawismus" bietet SHAKIBI, Pahlavissm, ders., Politics of Occidentalism, S.127-52.

${ }_{240}$ Vgl. Tehran \#A-324, 28.11.62, 788.00/11-2862, CF 60-63.
} 
Landreform. Arsanǧāni verblieb auch nach dem Rücktritt Aminis im Kabinett und konnte mit Unterstützung des Schahs seine umfassenden Reformen fortsetzen. Holmes sah in der „Bauernbefreiung“ die Grundlage für eine „,new political force“, was mit einer grundsätzlichen Schwächung der bisherigen landbesitzenden Eliten einherginge. Die neue Klasse der nun landbesitzenden Kleinbauern sollte den Grundstock für die persönliche politische Macht des Schahs bilden. Holmes aber zweifelte, dass, the people who have so long governed Iran as if the peasants who make up the majority of the population did not exist as human beings can successfully establish a new relationship with an emerging new peasant political force [...].“241 Für die nun anstehenden Wahlen zum 21. Mağles - Ministerpräsident 'Alam hatte Wahlen für den Sommer 1963 angekündigt - rechnete der Botschafter mit einer eher begrenzten Zahl Repräsentanten ländlicher Herkunft. Das Regime werde jedoch wenigstens eine symbolische Repräsentation mittels Wahlmanipulationen sicherstellen. Die Entscheidung zugunsten von Mağles-Wahlen war zumindest in Teilen eine Reaktion auf die zunehmende Kritik am konstitutionell fragwürdigen Regieren ohne Parlament seit dem Mai 1961, ein Vorwurf, den sich insbesondere eine sich neu formierende Allianz zwischen bedrohten Landbesitzen und reformfeindlicher schiitischer Geistlichkeit zu eigen gemacht hatte. ${ }^{242}$

Das State Department bestätigte einmal mehr die wiederhergestellte zentrale Rolle des Schahs in der amerikanischen Iranpolitik. Assistant Secretary Phillips Talbot pflichtete der Analyse des Botschafters bei und sagte für die Zukunft voraus:

The key seems to be the Shah's determination to exercise his power and influence and the continuance of his present mood of self-confidence. If he can keep his head and avoid being panicked into either too overtly rigged an election or an impulsive reaction to the criticisms and, perhaps, incidents that will inevitably follow any kind of an election, he should have much smoother sailing. ${ }^{243}$

In der Tat kündigte der Schah gegenüber Holmes seine Entschlossenheit an, Reformprogramm wie auch die Wahlen energisch durchzuführen - da Iran noch nicht die nötige Reife für wirklich freie Wahlen besäße, wolle er diese vorsichtig „lenken“, „[...] with guidance going especially to peasants who would be freed from landlord control.“ Zudem spielte er nun auch mit dem Gedanken, nach Vorbild de Gaulles ein nationales Referendum über das Reformprogramm abhalten zu lassen. ${ }^{244}$ Die frühen Errungenschaften der „Revolution von Oben“ wurden von der iranischen Führung von Anbeginn an auch öffentlich propagiert. Eine vorgeblich von Mohammad Rezā selbst verfasste Propagandaschrift „Die Weiße Revolution“ (enġelāb-e sefid)

\footnotetext{
${ }^{241}$ Ebd.; 'Alam hatte die Botschaft informiert, die Wahlen würden erst stattfinden, nachdem die erste Phase des Landverteilungsprogramms abgeschlossen sei. Aufgrund des Widerstands der schiitischen Geistlichkeit gegen das Frauenwahlrecht sollte dieses erst in der darauffolgenden Wahl zur Anwendung kommen. Vgl. Tehran \#A-295, 15.11.62, 788.00/11-1562, CF 60-63.

${ }^{242}$ Vgl. Tehran \#A-318, 26.11.62, 788.00/11-2662, CF 60-63.

${ }^{243}$ Talbot Letter, 7.12.62, ,I. The Shah \& Court (and Govt) 1962‘, NEA, GTI: RIAD, 1958-63, Box 4.

${ }^{244}$ Zitat aus Tehran \#A-319, 26.11.62, 788.00/11-2662, CF 60-63; gegenüber Harrison äußerte der Schah, dass ,[...] there was no constitutional provision for a referendum, but that, in fact, nearly every recent act of government had been outside the Constitution."; Harrison to FO, No. 1014, 29.11.62, PREM 11/3845, TNA:PRO.
} 
wurde 1965 in Teheran veröffentlicht und anschließend in eine Reihe anderer Sprachen übersetzt. ${ }^{245}$

Die Begeisterung des State Departments über die positive Übernahme der Reformer-Rolle durch den Schah wurde anfangs im Weißen Haus so nicht geteilt. Komer reagierte auf die Neuigkeiten mit dem Hinweis, die eigenen Analysten hätten bislang in der weitgehend als apathisch eingeschätzten Landbevölkerung kein bedeutsames Problem gesehen: „But the main problem is in the cities. Here not land reform, but economic growth and development is germane." Tatsächlich hatte Holmes selbst eingestanden, dass die 'Alam-Regierung das Interesse ihrer Vorgängerin an „long-range development“ zugunsten kurzfristiger Effekte und Budgetstabilität aufgegeben habe. ${ }^{246}$ Das Office of Greek, Turkish, and Iranian Affairs (GTI) im State Department bereitete die Antwort Talbots auf Komers Fragenkatalog minutiös vor. So sei wirtschaftliche Entwicklung inzwischen nur eines von drei ,high-priority U.S. objectives“ neben dem Erfolg der Landreform und, inzwischen völlig unverblümt im Sinne der Guidelines vom September 1962, dem Machterhalt des Schahs. Letzteren suchte man nicht mehr durch Schaffung neuer politischer Synthesen oder Einbeziehen der Opposition zu gewährleisten, sondern durch direkte Unterstützung für Militär, Polizei, Gendarmerie und durch Maßnahmen im Bereich der Zivilhilfe. Komer habe recht, ökonomische Entwicklung sei weiter von großer Bedeutung - allerdings müsse diese angesichts ihrer nur längerfristig spürbar werdenden Vorteile gegenüber dem mittelfristigen Gelingen der zentralen Landreform vorerst zurückstehen. ${ }^{247}$ Wie bereits in den Vormonaten zu registrieren, waren die internen Analysen der Situation aber weitaus pessimistischer, als es die optimistischen Berichte an das Weiße Haus glauben ließen. Noch skeptischer als Holmes sah man in GTI die neuen politischen Dynamiken, die das Landreform-Programm in Iran entfalteten. Mohammad Rezā werde es nicht gelingen, so befürchtete man, die Unterstützung der nationalistischen Opposition zu erlangen, sich aber gleichzeitig mit der alten Elite überwerfen - eine Koalition beider gegen den Thron könnte die Konstellation der frühen Mosaddeg-Zeit neu beleben. Andererseits habe sich der Schah aus dem bisherigen politischen Korsett befreit: „In essence, the Shah has, by his bold moves on land reform and his clean break with the old elite, increased the short-run dangers to his regime and has greatly improved his chances for breaking out of what had been a steadily worsening impasse. “248

\footnotetext{
${ }^{245}$ Vgl. PAHLAVI, White Revolution; in seiner eine (ersten) Autobiographie hatte der Schah bereits Kapitel über die Frauenemanzipation und die Landreform eingefügt. Vgl. PAHLAVI, Mission, S. 195-217; in gewisser Weise ging die Vermarktung der Pahlawi-Monarchie als zentrale Reforminstitution der eigentlichen Umsetzung der Reformprogramme voraus.

${ }^{246}$ Komer Memo, 4.12.62, ,Iran, 1961-1962, White House Memoranda', NSF, RWK, Box 424, JFKL; Holmes' Einschätzung in \#A-319. Siehe oben, Anm. 244.

${ }_{247}$ Bracken Memo, 10.12.62, ,Office Memoranda, 1962‘, NEA, GTI: RIAD, 1958-63, Box 4; GTI betonte, der Schah benötige die Unterstützung der Sicherheitsorgane, ,[...] until he has massive organized support from the rural and urban proletariat.“

${ }^{248}$ Ebd.; der Schah gebe im Grunde den lange Zeit erhobenen US-Forderungen nach einem Bruch mit der traditionellen Elite nun nach: „He has taken our advice literally, has thrown a wild forward pass, and the ball is in the air."; tatsächlich entsprach die neue Strategie des Schahs gerichtet auf Ko-Optation der Landbevölkerung und städtischer Unterschichten in wichtigen Teilen den Vorstellungen, die in den Task Force-Beratungen im Herbst
} 
Der Desk Officer für Iran in GTI, John W. Bowling, spekulierte in einem langen Memorandum Ende 1962 über „Future Problems in Iran“. Die Landreform sei nun ein „fact of life“ und werde die inneriranische Machtkonstellation nachhaltig ändern:

\begin{abstract}
Everything depends on the political success of land reform, which means that everything depends on whether or not the peasants' enthusiasm for their revolutionary new status can be maintained and that they will remain grateful to and confident in the Shah whom they identify with the change. Whether Iran's GNP rises or falls, whether the country has a good Plan \{gemeint ist der nächste Entwicklungsplan; R.P.\}or no plan at all, whether the economy goes statist or private, are all side issues now. Land reform, and the status of the peasantry thereafter, is everything. Ninety percent of the educated people in Iran, reactionary or Mosadeqist, National Front or communist, are bitterly opposed to the program and would like nothing better than to sabotage it, no matter what the consequences. The Shah can use the emancipated peasantry to counterbalance them, but only if the peasantry feels that land reform has helped them. The chips are on the table in plain sight. Side bets are irrelevant. ${ }^{249}$
\end{abstract}

Angesichts der zentralen politischen Bedeutung, die Bowling dem Erfolg oder Misserfolg der Landreform zumaß, mussten seine Vorhersagen beunruhigend wirken. Aufgrund der strukturellen Schwächen in Iran müsse innerhalb eines Jahres mit dem Kollaps der neu gegründeten Kooperativen gerechnet werden, da diese nicht in der Lage sein würden, die funktionalen und sozialen Aufgaben der traditionellen Landbesitzer und deren Agenten adäquat zu ersetzen. Im Anschluss könne nur staatliche Intervention den Zusammenbruch der ländlichen Wirtschaft mit nicht absehbaren politischen Auswirkungen verhindern - was eine Reorientierung der staatlichen Wirtschaftspolitik und, sehr wahrscheinlich, massiven Druck auf das Ölkonsortium zur Verbesserung der Einnahmesituation zur Folge haben dürfte: „What all this will come to is that some time within the next 18 months we are going to have to make a choice between finding about $\$ 75$ to $\$ 100$ million for these cooperatives in a way which will violate every AID criterion we have, or heading directly for a catastrophe in Iran. “ 250

William Polk vom Policy Planning Council, ein bürokratischer Verbündeter der KomerHansen-Linie, konstatierte in einer Analyse der neuen Schah-Strategie, dass diese - bei allen offenkundigen Gefahren - ,[...] offers the best hope we have of moderate and controlled evolution in Iran.“251 Polk empfahl eine Aufstockung der Nahrungsmittelhilfe im Rahmen des PL-480-Programms, um zu erwartende Produktionsausfälle aufzufangen, Unterstützung bei der

1961 entwickelt worden waren. Vgl. NEA Study Draft, o.D., ,20 Task Force on Iran`, NEA, GTI: RIAD, 195863, Box 3.

${ }^{249}$ Bowling Memo, 21.12.62, ebd.

${ }^{250}$ Ebd.; eine interne GTI-Bestandsaufnahme der eigenen AID-Strategie konstatierte eine ,reaction against comprehensive development planning and administrative reform" seit dem Amtsantritt der 'Alam-Regierung. Der 3. Plan sei bereist um 25\% reduziert worden. Sinnvoll sei die Multilateralisierung der Entwicklungshilfe. Die Weltbank könne besser Druck auf die iranische Zentralgewalt ausüben, ohne dass dies sofort als Intervention gewertet werde. Memo ,Discussion Paper on AID Policy Toward Iran', 10.1.63; Talbot Memo, 19.1.63, ,AID 1.General Policy. Plans. Coordination', NEA, GTI: RIAD, 1958-63, Box 8.

${ }^{251}$ Vgl. Polk Memo, 18.12.62, ,Iran', Lot 69 D 121, S/P Records: 1962, Box 216; dieses Polk-Memorandum wurde offenbar weit verbreitet. Es findet sich auch in den Central Files des State Department, in den Akten des Assistant Secretary for International Security Affairs im Department of Defense und in den National Security Files des Weißen Hauses. 
Ausbildung des Personals der neuen landwirtschaftlichen Kooperativen, Hilfe bei der Produktion von passenden Konsumgütern für die neue freie Bauernschaft und die Einrichtung einer zwischenbehördlichen Arbeitsgruppe zur Beobachtung und weiteren Analyse der Vorgänge im iranischen Agrarbereich. ${ }^{252}$ In der Tat schritten die Pläne des Schahs in der Folgezeit in Windeseile voran. Anfang Januar 1963 hatte sich der Schah nun für ein baldiges nationales Referendum über wichtige Teile des Reformprogramms, vor allem der Landreform selbst, entschlossen. Eine weitere Ausdehnung der Landreformgesetzgebung löste Proteste kleiner Landbesitzer, unterstützt von der Geistlichkeit, aus. Zugleich wuchs die Besorgnis über negative Reaktionen in den Stammesgebieten wie z.B. im traditionellen Herrschaftsgebiet der Gašğāei ${ }^{253}$

Trotz aller Warnzeichen verwandelte der Schah das Referendum in eine Demonstration seiner sozialrevolutionären Absichten. Landwirtschaftsminister Arsanğāni beschuldigte in einer Rede vor 25.000 Demonstranten die Nationale Front und die Mullahs, die Durchsetzung des Wahlrechts für Frauen zu sabotieren und kündigte - entgegen einer früheren Absprache mit der Geistlichkeit - die Zulassung von Frauen bereits für die kommende Abstimmung an. Versuche der Nationalen Front, das Referendum zur Abhaltung von Demonstrationen zu nutzen, wurden durch die Sicherheitskräfte mit repressiven Maßnahmen verhindert. Stattdessen organisierte die Regierung Großdemonstrationen von Unterstützern des Reformprogramms - Zehntausende Bauern wurden mit Bussen nach Teheran und in andere Städte transportiert. Gedungene Schlägerbanden (čāg்kešān) aus Südteheran und pro-Referendum-Demonstranten, mit Rückendeckung des erneut für solche Zwecke eingesetzten Fallschirmjägerbataillons, attackierten in der Hauptstadt Studenten und Aktivisten der Nationalen Front. ${ }^{254}$ Das durchorganisierte und von umfassenden Propaganda- und Mobilisierungsmaßnahmen geprägte Referendum vom 26. Januar 1963 brachte das gewünschte Ergebnis. Laut offizieller Verlautbarung stimmten 5.598.711 Stimmberechtigte für das Reformprogramm. Offiziell gab es nur 4.115 Gegenstimmen, was somit eine Zustimmungsrate von 99,9\% ergab. Angesichts der Umstände der Wahlabgabe - es gab unterschiedliche Wahlurnen für Ja- und Nein-Stimmen - war das einseitige Ergebnis wenig überraschend. Allerdings wies eine eingehende Analyse von INR im State Department darauf hin, die Begeisterung in Iran für die Landreform sei authentisch und die, wenn auch vorerst nur symbolische, Ausweitung des Wahlrechts auf Frauen könne durchaus im Sinne einer neue politischen Synthese in Unterstützung der Machtposition des Schahs verstanden werden. Bei allem Enthusiasmus stände die Umsetzung der Landreform vor gewaltigen administrativen, finanziellen und technischen Herausforderungen, die bislang kaum angegangen worden seien. Trotz der Richtungskämpfe

\footnotetext{
${ }^{252}$ Vgl. ebd.; im Sinne seiner Vorschläge unternahm Polk im März 1963 eine Erkundungsreise nach Iran und sammelte Informationen über die Umsetzung der Landreform. Siehe Polk Letter, 3.3.63, ,Iran 1/63-11/63', NSF, RWK, Box 424, JFKL; vgl. auch Talbot Memo, 7.1.63, FRUS 61-63, XVII-XVIII-XX-XXI Suppl., Dok. 106.

${ }^{253}$ Vgl. Tehran \#A-420, 5.1.63, 788.00/1-562; Meshed \#A-15, 24.1.63, 788.00/1-2463; CF 60-63; SANA Tehran \#89, 31.12.62, ,Iran General 12/62', NSF, Countries, Box 116A, JFKL.

${ }^{254}$ Vgl. Tehran \#629, 23.1.63, ,Iran General 1/63', NSF, Countries, Box 116A, JFKL; Tehran \#631, \#637, $24-$ 26.1.63, 788.00/1-24-2663, CF 60-63; zu Arsanǧāni vgl. auch ASHRAF, White to Islamic Revolution, S. 25f.
} 
und des mangelnden Zusammenhalts innerhalb der Nationalen Front sei die städtische Opposition weiterhin existent und würde bei absehbaren Schwierigkeiten in der Umsetzung des Reformprogramms sicherlich wieder aktiv werden. Weniger bedrohlich, so INR in fast schon teleologischem Selbstverständnis fortschreitender sozialer Modernisierung, sei die neu geschaffene Gegnerschaft konservativer und religiöser Kräfte, die grundsätzlich promonarchisch eingestellt und zudem eine „,dying force in Iran“ seien. ${ }^{255}$

Die positive Grundeinschätzung der Reformschritte Mohammad Rezās breitete sich auch in Washington aus. Bob Komer zeigte sich Mitte Januar von der Ernsthaftigkeit der monarchischen Reformschritte überzeugt: „He’s obviously bought Arsanjani’s idea of building a political base among the peasantry, and decided he'll have his own revolution without US advice.“256 Das State Department fasste die jüngsten Ereignisse in einem Memorandum an das Weiße Haus über „Recent Changes and Opportunities in Iran“ zusammen:

During recent months the Shah has launched and pushed with boldness and determination a reform program which is drastically and irrevocably altering the political situation and prospects of Iran. Much of the earlier context and background of Iranian politics has disappeared, and the political process has moved into a new background, with new forces operating within new parameters. Only the most general outline of the future can be predicted, for the Shah's politicoeconomic experimentation is without precedent. ${ }^{257}$

In einer erstaunlichen Re-Interpretation des im Kern gescheiterten reformistischen Interventionismus der USA in den vergangenen zwei Jahren führte man nun den Sinneswandel des iranischen Monarchen auf die eigenen Anstrengungen zurück. Zwar seien viele der von den USA angeregten politischen und wirtschaftlichen Reformanstrengungen versandet oder gescheitert, die grundsätzliche Idee der Notwendigkeit umfassender Reformen aber sei weiterhin lebendig geblieben. Vor allem die Gespräche während seines Staatsbesuchs in den USA im April 1962 und der Besuch des US-Vizepräsidenten hätten in Mohammad Rezā die Überzeugung reifen lassen ,[...] to set on his new and uncharted course.“ Die „Revolution von Oben“, so das State Department, sei bereits jetzt unumkehrbar. Entscheidend seien - hier spiegeln sich die früheren Ideen Holmes' und Polks wider - das Gelingen der eigentlichen Landreform und die Aufrechterhaltung von Ruhe und Ordnung auf dem Land. Amerikanische Einflussmöglichkeiten auf den angestoßenen Prozess seien begrenzt, abgesehen von

\footnotetext{
${ }^{255}$ Vgl. Tehran \#645, 28.1.63, 788.00/1-2863, CF 60-63; formal stand ein Gesamtpaket von sechs Reformschritten zur Abstimmung. Neben der (im Januar auf alle Ländereien von Großgrundbesitzern ausgedehnten) Landreform waren das die Regelung der Kompensation der Enteigneten (durch Erwerb von Anteilen einer zu gründenden Körperschaft für den gesamten im Staatsbesitz befindlichen Industriesektor), die Profitbeteiligung der Arbeiterschaft, eine Alphabetisierungskampagne, die Sozialisierung der Forste und eine Wahlrechtsreform, die die Einrichtung von Aufsichtsgremien in den Provinzen vorsah. Vgl. Hilsman Memo [RNA-6], 11.2.63, FRUS 61-63, XVII-XVIII-XX-XXI Suppl., Dok. 104; tatsächlich stand das Endergebnis vielerorts bereits im Vorfeld fest. An den Wahllokalen gab es keine Wahllisten und mehrfache Stimmabgabe „,was not only possible but encouraged.“; Isfahan \#A-29, 29.1.63, 788.00/1-2963, CF 60-63; bezeichnend für den Tenor des Gesamtwerks die Einschätzung bei KÜNTZEL, Die Deutschen und der Iran, S. 88, eine „klare Mehrheit der Iraner“ habe das Reformprogramm des Schahs unterstützt.

${ }^{256}$ Komer Memo, 15.1.63, ,Iran General 1/63', NSF, Countries, Box 116A, JFKL.

${ }^{257}$ Brubeck Memo, 21.1.63, FRUS 61-63, XVIII, S. 311.
} 
fortwährender Ermunterung und rhetorischer Unterstützung. ${ }^{258}$ Präsident Kennedy befolgte zumindest diesen Rat umgehend und gratulierte, trotz der offenkundigen Manipulationen, dem Schah zum erfolgreichen Ausgang des ,historischen“ Referendums: „It is [...] gratifying to learn that a vast majority has supported your leadership in a clear and open expression of their will.“ Auf iranischen Wunsch wurden der Inhalt des Telegramms und die Antwort des Schahs bald darauf veröffentlicht. ${ }^{259}$ Kennedys Nachricht - bezeichnenderweise vom ehedem überzeugten reformistischen Interventionisten Komer lanciert - nachvollzog nun auch sprachlich die seit einem Jahr vollzogene Abkehr vom Task Force-Programm und die mehr und mehr kritiklose Anbindung der US-Politik an die Pahlawi-Monarchie. Begeistert von der neuen Linie lud der Schah Kennedy sogleich zu einem Besuch in Iran ein. Komer selbst hatte sich inzwischen zum Befürworter einer Schah-geführten „Revolution von Oben“ gewandelt: „We may raise eyebrows at certain aspects of his current program but we're going to have to ride this tiger." 260

Ein solcher Aspekt war, dass die 'Alam-Regierung das Rapprochement mit der Nationalen Front nun aufgab und wieder zum ausschließlichen Mittel der Repression griff. 'Alam zeigte sich anfänglich zur Überraschung vieler weitaus liberaler gegenüber der nationalistischen Opposition als sein Vorgänger im Amt des Ministerpräsidenten. Nicht nur hatte er sämtliche Führungsfiguren der Nationalen Front aus dem Gefängnis entlassen. Er stattete sogar Allāhjār Sāleh einen persönlichen Besuch ab, erbat Zustimmung und Unterstützung für das eingeleitete Reformprogramm und bot der Front offenbar sogar einige Kabinettsposten in seiner Regierung an. Das Rapprochement scheiterte an den nationalistischen Forderungen einer Einhaltung der Verfassung, der Durchsetzung einer konstitutionellen Monarchie und sofortigen Wahlen. ${ }^{261}$ Wenige Tage vor dem Referendum wurden sodann die wichtigsten Führer der Zweiten Nationalen Front, Sāleh, Šāpur Baxtijār, Dārijuš Foruhar und viele andere, sowie die wichtigsten Studentenführer, erneut verhaftet. Die Verhaftungen und die Angriffe auf die nationalistische und religiöse Opposition während des Referendums weckten Besorgnis in Washington, dies könne „extremistische Reaktionen“ auslösen und man riet daher dem Schah zu Zurückhaltung. ${ }^{262}$ Mohammad Rezā jedoch signalisierte seine Entschlossenheit, den

\footnotetext{
${ }^{258}$ Ebd., Zitat auf S. 312; Komer versuchte erneut, die Gelegenheit für die Formulierung eines ,action program“ für Iran zu nutzen, doch Sicherheitsberater Bundy gab nur halbherzige Unterstützung. Vgl. Komer Memo, 23.1.63; Draft Bundy Memo, n.d., ,Iran, 1/63-11/63 (Folder 2 of 2)‘, NSF, RWK, Box 424, JFKL.

${ }_{259}$ State \#560, 29.1.63, FRUS 61-63, XVIII, S. 334; angesichts der gewählten Formulierung kann es nicht überraschen, dass der Schah höchst erfreut antwortete und zudem den Wunsch nach Veröffentlichung des Schriftwechsels aussprach - die Administration willigte ein. Siehe ebd., S. 334 n2; Tehran \#656, 31.1.63, 788.00/13163, CF 60-63; Office of the White House Press Secretary, Press Release, 13.2.63, ,Iran, Security 1961-1963', POF, Countries, Box 119, JFKL.

${ }^{260}$ Komer Memo, 29.1.63, ,Iran General 1/63‘; zur Einladung vgl. Holmes Letter, 7.2.63, ,Iran General 2/63‘, NSF, Countries, Box 116A, JFKL.

${ }^{261}$ Vgl. FlOOR, Revolutionary Character, S. 510f.; KedDIE, Roots of the Ulama's Power; Zonis, Political Elite, S. 73ff.; COTTAM, Nationalism in Iran, S. 305f.; KATOUZIAN, Struggle for Power, S. 242f.; Katouzian behauptet, Mosaddegs persönliche Intervention habe die Verhandlungen mit 'Alam beendet; vgl. Gratian Yatsevitch OHI, 5.11.88, OHIC; S. I-95.

${ }^{262}$ Vgl. CIA Information Report, 23.1.63, ,Iran General 1/63،, NSF, Countries, Box 116A, JFKL; das State Department riet zu einem ,,moderate course“" gegenüber der nichtkommunistischen Opposition und warnte, es sei ,possibly counter-productive, for him to repress opposition in ways suggestive of dictatorial approach."; State \#553, 28.1.63, FRUS 61-63, XVIII, S. 328; vgl. ebd., S. $328 n 4$.
} 
nationalistischen Führer dieses Mal den Prozess zu machen, und ließ sich auch von halbherzigen Interventionen Botschafter Holmes' nicht von diesen Plänen abbringen. ${ }^{263}$ Parallel setzten die Sicherheitsorgane die Unterdrückung der immer aktiver werdenden Geistlichkeit fort. Der Schah reiste demonstrativ in das geistliche Zentrum von Ġom - 'Alam erklärte gegenüber dem Botschafter , [...] that the political power of the Mullahs in any national terms had been destroyed.“264 Des Weiteren ging der Schah gegen die verbliebene Autonomie der Stämme vor und versuchte deren allgemeine Entwaffnung und Zwangsansiedlung im Kontext der Landreform durchzusetzen. Im März kam es daraufhin zu ersten Gefechten zwischen kleineren Stammesföderationen und der Gendarmerie in der Provinz Fārs - die größte der Ġašğāe i verhielt sich jedoch noch ruhig. ${ }^{265}$

Die CIA beschwichtigte in einem Bericht zum Zustand der Aufstandsbekämpfung im April 1963. Zwar erweise sich die Niederschlagung der Stammesunruhen als schwieriger als zuerst angenommen, doch handele es sich nicht um einen koordinierten oder organisierten Aufstand aller Stämme. Mittelfristig würden die iranischen Sicherheitskräfte wieder Herr der Lage werden. Als beunruhigender bewertete man die fortschreitende Mobilisierung religiöser Kräfte im Widerstand gegen das Reformprogramm. Zwar fänden solche „Elemente“ kaum Unterstützung in der breiteren Öffentlichkeit, ,[...] but they have the capability for isolated, well-organized acts of terrorism which would be carried out by fanatic followers."266 Beratungen in der Special Group (CI) illustrierten eine gewisse Besorgnis über die fortwährenden Unruhen im Süden und über eine mögliche Eskalation der Lage im Falle eines Übergreifens auf die kurdischen Gebiete. ${ }^{267}$ Gleichzeitig sah man den Aufstand in Fārs als ideale Gelegenheit, die neu erlernten Kenntnisse im Bereich der Counterinsurgency umzusetzen - ein weiterer Beleg für die fortschreitende einseitige Konzentration auf das Militärische im Rahmen der OIDP.

Der erste Progress Report zum CIDP für Iran, formuliert vom Country Team in Teheran im

\footnotetext{
${ }^{263}$ Vgl. Tehran \#670, 5.2.63, FRUS 61-63, XVII-XVIII-XX-XXI Suppl., Dok. 102; ,Insgesamt muß eingeschätzt werden, daß die Nationale Front von der Massenbewegung für die Reform überfahren wurde."; so eine interessante ostdeutsche Einschätzung. Siehe Bericht über die innenpolitische Entwicklung im Iran von 1961 bis Januar 1963, o.D. [1963], MfAA C 541/75, PAAA.

${ }_{264}$ Tehran \#A-486, 31.1.63, 788.00/1-3163, CF 60-63; offenbar hatte Arsanğāni im Falle der Fortsetzung der Proteste mit der Enteignung aller waqf-Ländereien gedroht. Siehe Lorenz Memo, 29.11.62, ,14. Opposition Movements‘, NEA, GTI: RIAD, 1958-63, Box 7.

${ }^{265}$ Vgl. Hilsman Memo [RNA-15], 26.3.63, ,Iran, 1/63-11/63 (Folder 2 of 2) ${ }^{‘}$, NSF, RWK, Box 424, JFKL; CIA, Office of Current Intelligence Special Report, 17.5.63, ,Iran General 5/63', NSF, Countries, Box 116A, JFKL; im kurz darauf veröffentlichten SNIE kamen die Geheimdienste zum dem Schluss, dass obgleich ,the modernization of the Iranian tribes is likely to be a long, sad, and brutal business, it is unlikely to pose any serious threat to national stability.“; CIA, SNIE 34-63, 10.4.63, FRUS 61-63, XVIII, S. 463; vgl. Malek Gašğā'i Letter, 2.4.63, ,Ghashghai, Malek Mansour, 1953-1973،, Box 331, Douglas Papers, LOC.

${ }^{266}$ CIA; Office of Current Intelligence, , Counterinsurgency in Iran', 23.4.63, Iran General 4/21/63-4/30/63', NSF, Countries, Box 116A; vgl. CIA, Office of Current Intelligence, 24.4.63, ,Iran 1/63-11/63', NSF, RWK, Box 424, JFKL.

${ }^{267}$ CIA Veteran George Carroll warnte im selben Meeting, dass ,all the elements are present in Iran which would provide for a major explosion should they be brought together at the same time."; die Gruppe beschloss, dass man Kontakt zu Richter Douglas aufnehmen solle aufgrund dessen enger Beziehungen zu den Ġašğā $\mathrm{i}$, denen zudem Kontakte nach Moskau nachgesagt wurden. Vgl. Meeting Special Group (CI), 29.3.63; ,SPECIAL [...] 4/18/63', Lot 68 D 451, SG (CI) Records, Box 2.
} 
Februar 1963, hatte bereits die ,new and pressing internal security problems“ betont, die aus dem angestoßenen Reformprogramm resultierten. Umstandslos wurde über eine Vertiefung der Zusammenarbeit mit den Sicherheits- und Repressionsorganen in Iran berichtet, offenkundig auf eine möglichst effiziente Kontrolle und Unterdrückung der Opposition gerichtet. So habe man die Befähigung der städtischen Polizei zur Kontrolle von Demonstrationen und Bekämpfung von Krawallen durch umfassendes Training und weitere Motorisierung gestärkt. Moral und Mobilität der Gendarmerie seien stark verbessert. Die Lieferung von Ausrüstung und die Ausbildungshilfe hätten zudem SAWAKs Möglichkeiten zur Kontrolle „subversiver Tätigkeiten“ professionalisiert. Auch von gemeinsamer amerikanisch-iranischer Notfallplanung für Sicherheitsmaßnahmen in Iranisch-Kurdistan war die Rede. Für die Zukunft ergäben sich neue Herausforderungen auf dem Land: Angesichts nicht auszuschließender Unruhen auf dem Land empfahl man die Entsendung von fünf zusätzlichen Experten für ,riot control“ an die amerikanische Gendarmeriemission: „It is particularly important for the stability of the central Government that it improve $\{$ sic $\}$ its capacity to handle possible village disturbances at the lowest possible level of violence and that it keep such disturbances localized to the maximum extent possible." Zeitgleich waren vor Ort ein 51 Mann starkes amerikanisches Special Forces Mobile Training Team und weitere Berater mit der fortschreitenden Ausbildung der iranischen Armee in der Aufstandsbekämpfung beschäftigt. ${ }^{268}$ Die Special Group (CI) reagierte auf das persönliche Interesse des Präsidenten am Zustand des Hilfsprogramms für die nationale Polizei Irans mit einen Informationsmemorandum. Man habe im Anschluss an den Schahbesuch vom April 1962 das Hilfsprogramm erweitert mit Schwerpunkt auf die Befähigung der Polizei zur Bekämpfung von städtischen Krawallmachern und Demonstranten: ,With the delivery of this equipment, and an increased emphasis on training, the police in Teheran will be brought significantly closer to the stage where it can cope with any foreseeable urban civil disturbance short of a national revolution.“269

Somit verstärkte die Initiierung des Reformprogramms durch den Schah die bereits zuvor wirksam gewordene fortschreitende Abwendung von der ursprünglichen Task Force-Politik. Geblieben war das nun wieder größer gewordene Interesse an Reform, vor allem an der Landreform. Allerdings wurden zentrale politische Reformen hintangestellt bzw. überhaupt nicht mehr erörtert. Für Fragen der politischen Ordnung war nun einzig und allein der

\footnotetext{
268 Vgl. Johnson Memo, 27.2.63, ,SPECIAL [...] -3/7/63', Lot 68 D 451, SG (CI) Records, Box 2; das Memorandum von U. Alexis Johnson fasst allerdings nur den weiterhin klassifizierten Progress Report zusammen. Meeting Special Group (CI), 28.2.63, FRUS 61-63, XVIII, S. 439f.; Zitat aus Tehran \#A-701, 29.5.63, DEF 19-2 US-IRAN, CF 63; im Bereich der Zivilhilfe unterstützte das DOD Ausbildungsprogramme und medizinische Hilfe.

${ }^{269}$ Vgl. Johnson Memo, 7.3.63, Iran General 3/63', NSF, Countries, Box 116A, JFKL; tatsächlich erhöhte man die jährliche Subvention von $\$ 150.000$ um eine weitere halbe Million US-Dollar für die kommenden zwei Jahre. Johnson pries die neuen Fähigkeiten der Polizei an und erklärte stolz, dass die Polizei die Auseinandersetzungen am Rande des Referendums problemlos und ohne Todesfälle habe kontrollieren können. Vgl. Komer Memo, 8.3.63, ebd.; die Botschaft gemeinsam mit den Public Safety-Beratern drängte über die zugesagten Erhöhungen hinaus auf weitere Hilfen, z.B. für den Aufbau einer berittenen Polizei zur effektiveren Straßenkontrolle. Vgl. Tehran \#898, 18.4.63, POL 23-1 IRAN, CF 63.
} 
Counterinsurgency-Rahmen entscheidend. Die fundamental geänderten Umstände ließen aber aus Sicht vieler Offizieller eine Neuformulierung der eigenen Strategie notwendig erscheinen. Am 14. März 1963 ordnete Präsident Kennedy mit NSAM №228 eine Überprüfung der „Iranian situation“ an. Das State Department sollte klären, ob der bestehende Ansatz in Iran weiterhin gültig und umsetzbar sei und wie die USA auf das vom Schah initiierte Reformprogramm reagieren sollten. Die Schwerpunktsetzung auf das brach liegende wirtschaftliche Entwicklungsprogramm in NSAM N`228 deutet auf einen weiteren, wenn auch bescheidenen Versuch Komers hin, den Einfluss des Weißen Hauses auf die Iranpolitik wiederherzustellen - die eigentliche Initiative zum neuen NSAM ging aber offenbar vom Bureau of the Budget und Kenneth Hansen aus. ${ }^{270}$ Im April lag zudem ein neues NIE zu Iran vor. Die Geheimdienste sagten weitere wirtschaftliche Schwierigkeiten infolge der abrupten Kehrtwende in der iranischen Wirtschaftspolitik weg von langfristigen Planungsvorstellungen voraus, zudem eine Reduktion der landwirtschaftlichen Produktion infolge der Landreform, und warnten vor weiteren politischen Verwerfungen. Dennoch schätzte man die politischen Überlebenschancen des Schahs nun als recht gut ein. ${ }^{271}$

Die offizielle Antwort des Secretary of State auf das NSAM in einem dreiseitigen Memorandum vom 20. April 1963 skizzierte ähnlich wie das NIE die folgenschwere Transformation der Machtbasis für die Monarchie im Rahmen eines beschleunigten und direkt vom Hof gesteuerten Reformprogramms als den wichtigsten Wandel innerhalb Irans: „The Shah abandoned his traditional power base in favor of dependence on a combination of the military, peasantry, and urban lower middle class.“ Der revolutionäre Wagemut von Oben gehe aber zugleich mit einer schwerwiegenden wirtschaftlichen Rezession und der weitreichenden Aufgabe des zuvor verfolgten Wegs einer durch staatliche Institutionen gesteuerten rationalen und zentralen Wirtschaftsplanung und Finanzkontrolle einher. Letzteres bedeutete im Kern die Ablehnung der seit dem Zweiten Weltkrieg kontinuierlich von amerikanischer Seite gegebenen Ratschläge. Für die nähere Zukunft erwartete das State Department die erfolgreiche Umsetzung der Modernisierung der Streitkräfte bei gleichzeitiger Reduktion der Sollstärke im Rahmen des vom Schah im September akzeptierten Fünfjahres-MAP-Programms über \$300 Millionen. Das Militär werde aller Voraussicht nach loyal zum Schah bleiben, zumal ein Zusammenschluss (,in a way which could cause the Shah to fall“) der disparaten Opposition bestehend aus Geistlichen, den gebildeten städtischen Schichten und den enteigneten Großgrundbesitzern nicht zu erwarten sei - somit bleibe der Schah auf absehbare Zeit ,the ultimate repository of

\footnotetext{
${ }^{270}$ Vgl. NSAM N ${ }^{\circ} 228$, ,Review of Iranian Situation‘, 14.3.63, FRUS 61-63, XVIII, S. 424f.; die Nichterwähnung der Landreform und die herausgehobene Betonung des 3. Entwicklungsplans lassen eine Initiative Komers vermuten - bestätigende Dokumente für die Annahme finden sich jedoch nicht. Polk vom Policy Planning Council war bereits im März zur Erkundung nach Iran gereist und hatte offenbar persönlich eine Nachricht Rostows dem Schah und anderen iranischen Offiziellen überbracht. Offenbar ging es um eine Idee Rostows zur Unterstützung der Landreform durch den Aufbau eines nationalen Netzes von Kaufhäusern, um den steigenden Konsum der „befreiten“ Bauern zu befriedigen. Polk sprach ebenfalls von der Gelegenheit, ,,in pushing the development of an action program for Iran"; zur Initiative des Bureau of the Budget siehe Komer Memo, 16.5.63, ,Iran, 1/63-11/63, White House Memoranda'; siehe auch Polk Letter, 3.3.63, ,Iran 1/63-11/63', NSF, RWK, Box 424, JFKL.

${ }^{271}$ Vgl. CIA, SNIE 34-63, 10.4.63, FRUS 61-63, XVIII, S. 459-64.
} 
power in Iran“" und der Garant für die pro-westliche Orientierung des Landes und die enge Allianz mit den USA. Im Kern bestätigte die Antwort des State Department die bestehende Iranpolitik, empfahl sogleich für die Zukunft eine zielgesteuerte Unterstützung des monarchischen Reformprogramms, insbesondere die Ermunterung weiterer Reformschritte in der Fiskalpolitik und die Verbesserung des Umfelds für Investitionen und - vor allem punktuelle Hilfe bei den wahrscheinlich zu erwartenden Schwierigkeiten in der Folge der überstürzten Landreform. Als eine mögliche Schwerpunktsetzung wurde ein Ausbau der Nahrungsmittelhilfe im Rahmen des Food-for-Peace-Programms (PL-480) genannt. ${ }^{272}$

Ein beigefügtes umfassenderes Strategiepapier, betitelt „U.S. Strategy for Iran“, zeichnete ein detailliertes Bild der geänderten Lage in Iran und machte weitere Vorschläge für eine Adaption der US-amerikanischen Politik. In der Kernaussage ging die U.S Strategy mit ihrer nahezu bedingungslosen Akzeptanz der Führungsrolle des Schahs über die Guidelines vom September 1962 noch hinaus:

The U.S. has a large, shared interest with the Shah both in foreign and domestic political fields. While there are areas of divergence, e.g., about the initial speed of the land reform program and the role of the Third Plan in economic development, they are differences more of emphasis than of essence. The Shah's estimate of internal requirements for the political stability of his regime does not always coincide exactly with ours. However, the monarchy, which provides the stability not yet available through popular institutions or long popular experience in political affairs, is in fact the sole element in the country that can at present give continuity to public policy. The Shah, therefore, remains a linchpin for the safeguarding of our basic security interests in Iran. ${ }^{273}$

Das Strategiepapier gestand zwar Schwächen im bisherigen amerikanischen Ansatz ein. So habe man die Schwerpunktsetzung auf die Umsetzung des wirtschaftlichen Entwicklungsprogramms im Rahmen des 3. Plans mit dem Fall der Amini-Regierung aufgegeben. Und die Vereinbarung über das MAP-Programm sei eigentlich auf Basis einer zukünftigen Priorisierung des wirtschaftlichen und zuvorderst des industriellen Aufbaus erfolgt - stattdessen läge der Schwerpunkt nun unerwartet auf einer beschleunigten Landreform. Allerdings ergäben sich aus dem Reformprogramm Mohammad Rezās - dessen Ursprung man nicht zuletzt in den eigenen Reformratschlägen über die Jahre sah - längerfristig Chancen für größere Stabilität und Wohlfahrt in Iran. Für das State Department hatte sich die Bedeutung des vereinbarten MAP-Programms nun verschoben: Statt den Entwicklungsplan vor weiteren Militärausgaben zu schützen, diente es nun vorgeblich dazu, die finanziellen Mittel für eine

272 Vgl. Rusk Memo, 20.4.63, FRUS 61-63, XVIII, S. 477ff.; die Verabschiedung des endgültigen MAPProgramms durch den Präsidenten wurde formal zum Anlass für NSAM N 228 genommen. Siehe Chenery Memo, 25.1.63, FRUS 61-63, XVII-XVIII-XX-XXI Suppl., Dok. 101.

${ }^{273}$ Report ,U.S. Strategy for Iran‘, 20.4.63, ebd., Dok. 105 \{Hervorheb. v. Verf.\} [im Folgenden U.S. Strategy]; angehängt an das Strategiepapier sind unter anderem eine schablonenhafte Auflistung der gegenwärtigen Politik gegenüber Iran mit Schwerpunkt auf die außenpolitischen und militärischen Bereiche, die ursprüngliche Antwort AIDs auf NSC Action 2447 vom 8.3.62 (vgl. oben S. 236, Anm. 54, und S. 294, Anm. 5); sowie die eben verabschiedete Country Assistance Strategy for Iran von AID und eine Zusammenfassung der bisher geleisteten Militärhilfe. Die Anhänge befinden sich in ,Iran General 4/20/63‘, NSF, Countries, Box 116A, JFKL. 
gelungen Umsetzung der Reformen sicherzustellen. ${ }^{274}$ Trotz dieser Fehlannahmen in der bisherigen Politikformulierung seien kein Neuansatz - hier wieder die Sorge vor einer neuerlichen Iran Exercise, gelenkt vom Weißen Haus -, sondern nur Feinjustierungen am bisherigen Ansatz vonnöten. Grundlage seien die in der AID-Strategie für das Fiskaljahr 1964 vorgenommenen Anpassungen. Im Zentrum stände nun die amerikanische Unterstützung für das Reformprogramm: „U.S. support for the program is dictated by the fact that only by supporting it can we influence a broad and sweeping change which we could not effectively halt even if we wanted to. “275

Gleichzeitig warnte das Strategiepaper explizit vor den „Pitfalls of Direct Involvement“, eigentlich ein Frontalangriff auf die Grundannahmen hinter dem Ansatz des reformistischen Interventionismus. Eine direkte Einflussnahme auf die Grundsatzentscheidungen der Landverteilung und der Profitbeteiligungen für die Arbeiterschaft sei angesichts der innenpolitischen Brisanz dieser Fragen zu vermeiden. Dies sei eine „Iranian revolution which will evolve at a Persian tempo and produce Persian results“. Nur als ,indigenous effort“ könne die Reform Erfolg zeitigen - jeder Eindruck, der Prozess sei „American-dominated“ wäre in der öffentlichen Wahrnehmung fatal. ${ }^{276}$ Die Vorschläge des State Department und AIDs beschränkten sich daher auf begleitende Maßnahmen in der Umsetzung der Reformen wie eben den Ausbau des PL-480-Programms, um das Programm in ökonomisch möglichst sinnvolle und sozial gerechte Richtungen zu lenken und zugleich eigene politische Verantwortung für die wesentlichen Entscheidungen möglichst auszuschließen. ${ }^{277}$ Gleichzeitig empfahl man, die neue Prioritätensetzung der Iraner weg von zentraler Wirtschaftsplanung und Modernisierung weitgehend widerstandslos hinzunehmen, wobei man sich der zentralen Kehrtwende gegenüber der Task Force-Politik von 1961 durchaus bewusst war. ${ }^{278}$ In Revision der bisherigen Glaubenssätze betrachtete man nun retrospektiv die Reformen Aminis und seiner „Americantrained advisers“ als ,overtly ambitious and unrealistic in the political sense“. Die Idee einer beschleunigten wirtschaftlichen Entwicklung und Modernisierung, durchgesetzt über eine zentrale Steuerungsbehörde mit vollständiger Budgetkontrolle - zugleich eine ,excellent opportunity for the U.S. to exercise important influence“ - sei im Grunde mit der politischen Realität in Iran immer unvereinbar gewesen. ${ }^{279}$ Allerdings existiere der 3. Entwicklungsplan wenn auch in um ca. ein Viertel geminderter Größenordnung - weiterhin und sei bereits von

\footnotetext{
${ }^{274}$ Vgl. U.S. Strategy, S. 3: „Recent changes in Iran are linked, to some indeterminable extent, with U.S. programs and policies. They probably would have occurred in any case, though perhaps at a different time and in a different way."

275 Ebd., S. 5.

${ }^{276}$ Ebd., S. 6; dieser Teil des Strategiepapiers war direkt von einem Bericht der Botschaft übernommen worden, die sich noch expliziter gegen Interventionen ausgesprochen hatte. Vgl. Tehran \#A-618, 4.4.63, POL IRAN-US, CF 63.

${ }^{277}$ Neben der Ausweitung des PL-480-Programms zur Überbrückung möglicher Produktionsausfälle und zur Bereitstellung lokaler Währung zur Deckung der Kreditbedürfnisse der agrarischen Kooperativen schlug das Strategiepapier die Gewährung technischer Hilfe, Ausbildungsmaßnahmen sowie Darlehen für den Import landwirtschaftlicher Maschinen und Fahrzeuge aus den USA vor.

${ }^{278}$ U.S. Strategy, S. 8.

${ }^{279}$ Ebd., S. 10.
} 
der IBRD zustimmend geprüft worden. Zumal habe der Schah selbst eingesehen, so das Strategiepapier, dass von einer großen Steigerung des Militäretats abzusehen sei. Ausgaben in Höhe von etwa $25 \%$ des Haushalts für militärische Zwecke seien jedoch weiterhin notwendig „[...] in maintaining in power a progressive and pro-Western monarch over the next few years“. Für die nahe Zukunft sah man angesichts der verbesserten finanziellen Situation Irans keine Notwendigkeit für größere Wirtschaftshilfe oder Entwicklungsdarlehen - für das kommende Haushaltsjahr erwartete man allerdings einen Bedarf zwischen \$25 und 40 Millionen. ${ }^{280}$

Mit dieser U.S. Strategy for Iran warb das State Department für die Fortsetzung der bisherigen Politik gerichtet auf Abschreckung kommunistischer Aggression, Beibehaltung einer prowestlichen Orientierung Irans, Bewahrung der inneren Stabilität und Unterstützung der ökonomischen Entwicklung des Landes. Konkret beinhaltete die Zusammenstellung des State Department, bei leichten Anpassungen, die Fortsetzung der bisherigen Politik, vorwiegend orientiert an den Vorgaben des Schahs, bei weitgehender Vermeidung eigener Involvierung, also eine nahezu vollständige Aufgabe des reformistischen Interventionismus. Die kurz zuvor verabschiedete Country Assistance Strategy für Iran von AID sekundierte dieser Haltung. Moderate und punktuelle Unterstützung für das Reformprogramm, gerade für die Landreform, und begrenzte Hilfe für die Projekte im 3. Plan, wenn möglich unter Bedingung institutioneller Reformen, in einer Größenordnung bis maximal \$40 Millionen, waren beabsichtigt. ${ }^{281}$

Für die Komer-Hansen-Gruppe und die reformistischen Interventionisten war NSAM N²28 die letzte Gelegenheit, die von ihnen präferierte aktivistische Politik im Sinne ihres Action Program vom Oktober 1961 durchzusetzen. Bereits vor der offiziellen Antwort des State Department warnte Komer während einer Informationsreise in Iran, die Hauptgefahr für die Revolution des Schahs sei die weiterhin stagnierende wirtschaftliche Entwicklung. Angesichts der ökonomischen Schwäche im Zusammenspiel mit den $\mathrm{zu}$ erwartenden sozialen Verwerfungen infolge der schlagartigen Reformen drohe der Kollaps. Es gelte, so rasch als möglich die nationale Wirtschaft anzukurbeln. ${ }^{282}$ Die vorliegenden Dokumente legen allerdings die Vermutung nahe, dass es Bob Komer hier nicht in erster Linie um wirtschaftspolitische Fragen ging. Einer Anregung von Kenneth Hansen folgend wollte Komer das traditionelle Einflussmittel der zentralen Wirtschaftsplanung nutzen, um erneut für amerikanischen Aktivismus in Iran zu werben. Tatsächlich war die anhaltende Rezession in

\footnotetext{
${ }^{280}$ Ebd., S. 13f.; mit der Abschaffung direkter Budgetbezuschussung habe man einen wichtigen Hebel zur Einflussnahme auf iranische Politik eingebüßt, dafür aber Selbsthilfe und Eigenverantwortung auf Seiten der Verbündeten gestärkt; vorheriges Zitat auf S. 15.

${ }^{281}$ Vgl. Gaud Memo, 2.3.63, ,Iran General 4/20/63', NSF, Countries, Box 116A, JFKL; die AID Assistance Strategy stellte lapidar fest: ,the channels previously existing between the U.S. and GOI \{Government of Iran; R.P.\} for discussing economic development and reform issues have been severely limited."; laut einem Anhang hatte Iran in dem gerade zu Ende gegangenen Haushaltsjahr 1963 sogar 10 Millionen Budgetüberschuss erzielt. Siehe ,Iran's Central Government Finances, FY 1961-63‘, o.D., ebd.; zur US-Unterstützung für die Landreform vgl. Bowling Memo, 21.5.63, ,AID 1.[...] Coordination‘, NEA, GTI: RIAD, 1958-63, Box 8.

${ }^{282}$ Vgl. Beirut \#924, 10.4.63; in der State Department-Kopie des Telegramms hatte ein Offizieller den entsprechenden Absatz abfällig kommentiert: „,This is hardly the problem!“”; Beirut \#924, POL 1 IRAN, CF 63.
} 
Iran eher auf die unsichere Lage und ihre Auswirkungen auf die private Investitionsbereitschaft als auf eine allzu zurückhaltende Fiskalpolitik der Regierung zurückzuführen.

Kenneth Hansen hatte die Antwort auf NSAM N²28 scharf kritisiert als - wenn auch unausgesprochene - Abkehr von der vereinbarten US-Politik gegenüber Iran. Die in der Task Force vom Mai 1961 beschlossene Politik sei unzureichend umgesetzt worden - der einzige und bescheidene Erfolg sei die verabredete Reduktion der Sollstärke der iranischen Streitkräfte. Die vom Schah angestoßenen Reformen seien unüberlegt wie überhastet - es gäbe ernsthafte Bedenken, ob die Landreform überhaupt ein geeignetes Mittel sei, um der Monarchie eine neue massentaugliche Basis zu verschaffen. Die Ausweitung des politischen Bewusstseins durch die „Revolution von oben“ und die dadurch geweckten Erwartungen könnten wohl mittelfristig nur mit der Ausweitung der Repression durch die Sicherheitskräfte unter Kontrolle gebracht werden: ,If the land reform misfires, the result could be a new and powerful interaction between unrest in the countryside and in the cities." Angesichts dieser Gefahren sei eine tatkräftige und zielgerichtete Strategie mit Blick auf die Umsetzung der alten und weiterhin Gültigkeit beanspruchenden Ziele vom Mai 1961 geboten, mit dem Ziel eine „,base for more determined intervention“ in der Zukunft aufzubauen. ${ }^{283}$ Hieraus ergab sich für Hansen die fortgesetzte Notwendigkeit, wirtschaftliche Planung und die für ihn damit untrennbare Unterstützung der städtischen Mittelschichten zu betonen. ${ }^{284}$ Komer stimmte Hansens Einschätzung zwar zu, der Ansatz des State Department sei im Kern ,passiv und fatalistisch“, setzte sich aber zugleich entscheidend $\mathrm{ab}$ und sprach sich nunmehr gegen eine umfassende Revision der bestehenden Politik aus: „I see no other realistic alternative to backing the Shah's reforms (which have already proceeded too far to turn back), but attempting to guide it into more effective channels.“ Der wichtigste dieser Kanäle war aber auch für Komer eine erneute Schwerpunktsetzung auf Wirtschaftsplanung. Im Kern ging es ihm allerdings nicht wirklich um eine Rückkehr zur (über-)ambitionierten Task Force-Politik, sondern um die Rettung der Restbestände der reformistisch-interventionistischen Strategie der Vergangenheit. Komer selbst gestand ein, die Politikziele vom Mai 1961 seien seit langem überholt. Er plädierte für einen „taktischen“ Ansatz und argumentierte gegen die grundsätzliche Ablehnung direkter Involvierung, wie sie in der U.S. Strategy for Iran ausformuliert worden war:

We are directly involved in Iran; we will be blamed just as much if the Shah's revolution fails with us sitting on the sidelines as if it fails with us in there pitching. We just can't afford 19th Century diplomacy in Iran. ${ }^{285}$

Neben der altbewährten Reformisten-Gruppierung in Weißem Haus und dem Bureau of the Budget gab es weiterhin die - allerdings weitgehend ignorierten - „Douglasites“ sowie

\footnotetext{
${ }^{283}$ Hansen Memo, 7.5.63, ,NSAM 228, [...] 5/63‘, NSF, M\&M, Box 340, JFKL; Hansen schlussfolgerte: „It constitutes a change of policy although it is not so labeled." \{Hervorheb. i. Orig. $\}$.

${ }^{284}$ Ebd.

${ }^{285}$ Komer Memo, 16.5.63, FRUS 61-63, XVIII, S. 539f. \{Hervorheb. i. Orig.\}.
} 
„Minderheitenmeinungen“ im Defense Department und in der CIA, welche die Weiße Revolution für zu riskant hielten und amerikanische Interventionen befürworteten, um Schlimmeres zu verhindern. ${ }^{286}$ In der Special Group (CI) sprach sich Bob Komer folgerichtig dafür aus, dass , ,...] we must take a more active advisory role in the economic and fiscal policy planning of the Government of Iran." ${ }^{287}$ Die Gruppe beschloss, eine entsprechende Einschätzung der Botschaft in Teheran anzufordern. Holmes berichtete daraufhin über die Bemühungen der Botschaft, die iranische Regierung zu einer Abkehr des scharfen Austeritätskurses zu bewegen, und schlug auch einige zusätzliche Schritte zur amerikanischen Unterstützung der Landreform vor, blieb aber bei seiner prinzipiellen Ablehnung weiterreichender Einmischung: ,Revolutions can't be controlled by foreigners, and in trying to do so, we could endanger all we have gained in this country in the past ten years.“288

Bob Komer beabsichtigte, den erwarteten Widerstand der Berufsdiplomaten in einer Diskussion der offiziellen Antwort auf NSAM N ${ }^{\circ} 28$ in der NSC Standing Group zu brechen. ${ }^{289}$ Ein von ihm inspiriertes Memorandum des Sicherheitsberaters empfahl eine Beratung über die Implementierung der Ratschläge des State Department, also der Unterstützung des reformistischen Experiments des Schahs, zuvorderst die Frage eines möglichst konkreten Plan of Action. Das State Department beharrte dagegen bereits im Vorfeld auf die Vorteile „,normaler diplomatischer Prozeduren“ und die Einschaltung internationaler Organisationen wie z.B. der IBRD zur Unterstützung der iranischen Reformbemühungen. ${ }^{290}$ Während des Treffens der Standing Group beruhigten sich die Gemüter jedoch rasch wieder. Bob Komer und andere reformistische Interventionisten unterschieden sich in ihren Standpunkten in der Tat nur gering von denjenigen der Offiziellen des State Department. Grundsätzlich herrschte Einigkeit über die Notwendigkeit, zum Gelingen des monarchischen Reformprogramms beizutragen. In den verschiedenen Sachfragen konkreter Unterstützung wie der Landreform oder der zukünftigen Involvierung der Weltbank gab es ebenfalls kaum Gegensätze. Die Gruppe akzeptierte die Vorschläge des State Department weitgehend, das im Gegenzug die Ausarbeitung einer Umsetzungsstrategie durch das Country Team in Teheran in Aussicht stellte. Streit gab es tatsächlich nur in der Frage des anzustrebenden Ausmaßes amerikanischer Intervention im wirtschaftlichen Bereich. Assistant Secretary Talbot wiederholte einmal mehr

\footnotetext{
${ }^{286}$ Diese Charakterisierungen entstammen einem NEA-Memo für Governor Harriman. Dem Memo zufolge würde das Einschlagen der ..,Douglasite“-Richtung, also die erzwungene Machtübergabe an die Nationalisten zu einer zweiten Mosaddeg-Ära führen, „but with Communists having learned not to miss their cue.“; folge man den Empfehlungen der Minderheitenmeinungen in DOD und CIA, den Schah zur Einstellung der Reformen und zur Machtteilung mit Militär und traditioneller Elite zu zwingen, drohe eine Wiederholung der Zāhedi-Ära mit der mittelfristigen Drohung eines linksgerichteten Staatsstreichs. Die vom Bureau of the Budget geforderte Machtübergabe an die „Western-trained economists“ sei absurd, da diese keine politische Hausmacht besäßen. Memo for Harriman, 2.5.63, FRUS 61-63, XVII-XVIII-XX-XXI Suppl., Dok. 107.

${ }^{287}$ Meeting of the Special Group (CI), 2.5.63, FRUS 61-63, XVIII, S. 510.

${ }^{288}$ Tehran \#996, 15.5.63, ,Iran General 5/63', NSF, Countries, Box 116A, JFKL; die auslösende Anfrage in State \#811, 9.5.63, ebd.

${ }^{289}$ Die NSC Standing Group war eine Schöpfung McGeorge Bundys Ende 1961, um die Abläufe und die Arbeit des NSC effizienter zu gestalten. Ab April 1963 tagte die Gruppe unter seinem Vorsitz. Vgl. ed. note in FRUS 6163, XXV, S. 34f.

${ }^{290}$ Vgl. Bundy Memo, 17.5.63; ,NSAM 228 Iran: Comments on questions raised in Bundy Memorandum', 21.5.63, ,NSAM 228, [...] 10/63‘, NSF, M\&M, Box 340, JFKL.
} 
das Mantra des State Department: Es sei essentiell, dass ,,[... that the U.S. should not become involved in a revolution in Iran to the extent that our entire future relations with this country would be put in jeopardy if the revolution got out of control or if the Iranians turned against us because of our extensive intervention in their internal affairs. “291

Im Kern sah Talbot voraus, was mit der späteren iranischen Revolution von 1978/79 kennzeichnend für die weiteren iranisch-amerikanischen Beziehungen werden sollte. Allerdings war diese Bemerkung des Assistant Secretary in erster Linie taktisch intendiert, um weitere Task Force-Experimente im Ansatz zu unterbinden. Komers zentrales Argument, die USA würden aufgrund ihrer herausragenden Stellung in Iran und der historischen Rolle, die sie seit dem Zweiten Weltkrieg gespielt hatten, in jedem Fall für den Ausgang der Weißen Revolution verantwortlich gemacht werden, war fraglos plausibel. Zudem bezog sich Talbots Definition von „Intervention“ ausschließlich auf Versuche, zentrale politische Entscheide bzw. Personalentscheidungen zu beeinflussen. Er, aber auch Holmes und Andere, übersahen, dass die amerikanische Rolle beim Aufbau der Sicherheitskräfte und Repressionsorgane, materiell wie ideell, sowie die enge fortgesetzte Kooperation mit diesen, aus Sicht der breiten iranischen Bevölkerung ebenfalls und in hohem Maße interventionistisch war. Somit würden Talbots Befürchtungen über die Folgen einer zukünftigen Revolution auf das gemeinsame Verhältnis auch unabhängig von der Fortsetzung der von ihm kritisierten Form von Einmischung eintreffen.

Die Position von Kenneth Hansen und einiger weniger anderer Offizieller war inzwischen die von Außenseitern. Zwar bestanden einige der traditionellen Gegensätze zwischen Weißem Haus und State Department fort, doch war dies nicht mehr mit der Situation von 1961 oder 1962 zu vergleichen. Vom eigentlichen Task Force-Programm vom Mai 1961 war nur noch wenig verblieben - tatsächlich finden sich auch keine formellen Bezugnahmen mehr in den Akten. Zwei Jahre später sprach niemand mehr von einem starken Ministerpräsidenten unabhängig vom Hof, vom Zurückdrängen des Schahs in seine konstitutionelle Rolle oder der politischen Einbindung der nationalistischen Opposition. Die Weiße Revolution aber bewerteten nicht wenige als Umsetzung eines zentralen Bestandteils der ursprünglichen Kennedy-Politik - was Komer zwei Jahre zuvor als ,controlled [...] revolution as the only alternative to an uncontrolled“ skizziert hatte, wenn auch nun unter wesentlich geänderten Vorzeichen. ${ }^{292}$ Tatsächlich begann sich der Einfluss im bilateralen Verhältnis zwischen beiden Staaten langsam umzukehren. Über das bereits etablierte - und in Zukunft immer bedeutendere - pro-

${ }^{291}$ NSC Standing Group Meeting No. 6/63, 21.5.63, FRUS 61-63, XVIII, S. 553; siehe auch Gilpatric Memo, 22.5.63, ebd., S. 554; Talbot hatte im Vorfeld in einem weiteren Memorandum die Absegnung des NSAM N ${ }^{\circ} 228-$ Programms des State Department als ,a basic strategy guide“ erbeten. Vgl. Talbot Memo, 21.5.63, FRUS 61-63, XVII-XVIII-XX-XXI Suppl., Dok. 109; für eine wenig überzeugende Interpretation, die Landreform sei auf „perfide“ Politik der USA zurückzuführen, die den Schah zwangen „,into adopting measures against his better judgments“, vgl. MAJD, Resistance to the Shah, S. 130; GOODE, Reforming Iran, S. 25, interpretiert dieses Treffen als entscheidenden Wendepunkt in der Politik der Kennedy-Administration. Die vorliegende Studie datiert die Aufgabe des reformistischen Interventionismus aber bereits auf die Jahreswende 1961/62.

${ }^{292}$ Vgl. oben S. 260, Anm. 125. 
iranische Netzwerk der Pahlavists in Washington verstärkte bzw. lenkte Mohammad Rezā die amerikanische Unterstützung für sein Programm. Ein internes GTI-Memorandum vom Mai 1963 berichtet z.B. von einer Initiative Kermit Roosevelts zur Unterstützung für die Landreform in Iran durch private und staatliche Stiftungen und Organisationen. John Bowling - sichtlich irritiert durch das gleichzeitige und unverhüllte Verfolgen persönlicher wirtschaftlicher Interessen durch Roosevelt - warnte vor dessen Beziehungen $\mathrm{zu}$ hochgestellten Persönlichkeiten in Washington wie z.B. Averell Harriman. Man müsse daher jederzeit mit einem ,rush call“" rechnen. ${ }^{293}$

Die Debatte um NSAM N²28 bestätigte somit im Grundsatz den bestehenden Kurs, wie auch Komer eingestand: „In a nutshell, all of us agree that we are committed to the Shah's revolution and must do all we can to make it a success.“294 Die weiteren bürokratischen Auseinandersetzungen drehten sich in erster Linie um die Verbesserung der administrativen Fähigkeiten in Iran und die Unterstützung bei den anliegenden wirtschaftlichen und finanzpolitischen Folgeschritten. ${ }^{295}$ Komer versuchte weiterhin, seine Vorstellungen von angemessenen Interventionen durchzusetzen und kritisierte Holmes für seine Ablehnung entsprechender „Ratschläge“ an die iranische Adresse. ${ }^{296}$ Er drängte darauf, die notwendigen wirtschaftlichen Anpassungen und die amerikanische Rolle dabei zur Leitlinie des in der NSC Standing Group verabredeten, vom Country Team in Teheran zu formulierenden Action Program zu machen. ${ }^{297}$ Auf Betreiben von Komer und Averell Harriman wurde Botschafter Holmes zu Konsultationen nach Washington zurückbeordert. Ziel Komers war es, eine Rückkehr zum auflagenfreien „Schaufeln“ amerikanischer Hilfsleistungen an Iran zu verhindern und wenigstens den tradierten Einfluss auf die zentrale Wirtschaftslenkung zu bewahren sowie die iranischen administrativen und ökonomischen Schritte in die richtige Richtung zu lenken. Allerdings übersah Komer dabei den empfindlichen Einflussverlust, den die USA in den vorausgegangenen Monaten in Iran erlitten hatten. Die Abwertung der Planorganisation und die Ausschaltung des pro-amerikanischen Netzwerks in dieser und in anderen Behörden hatten einen der wichtigsten Hebel für amerikanische Einflussnahme beseitigt - US-Einfluss konzentrierte sich nun in erster Linie auf den Bereich der Sicherheitskräfte im Rahmen der Militär- und Gendarmeriemissionen und der geheimdienstlichen Verbindungen. Ausbildungshilfe, das MAP-Programm und die verschiedenen Programme zur Stärkung der inneren Sicherheit verschafften ebenfalls Zugang

\footnotetext{
${ }^{293}$ Roosevelt berichtete über Kontaktaufnahmen mit den Rockefeller und Ford Foundations und schlug als Liaison eine ,gemeinnützige“ Gesellschaft vor. Die Stiftungen beabsichtigten diese Gesellschaft für die LandreformUnterstützung zu beauftragen. Vgl. Bowling Memo, 24 May 1963, ,AID 1.General Policy. Plans. Coordination`, NEA, GTI: RIAD, 1958-63, Box 8.

${ }^{294}$ Komer Memo, 20.6.63, ,Iran', Box 473, Harriman Papers, LOC; bezeichnend für das nachlassende Interesse an Iran ist Harrimans handschriftlicher Vermerk neben NSAM N²28: „What is this?“.

${ }^{295}$ Komer drängte weiterhin auf ein ,concrete set of actions“, um die Iranpolitik weiter vom Weißen Haus beeinflussen zu können. Man entschied sich gegen eine exponierte Rolle der IBRD in der Landreform, unterstützte diese aber mit einem generösen PL-480-Programm. Vgl. Memo to Harriman, 13.6.63; Talbot Memo, 11.6.63, ,SPECIAL [...]6/13/63', Lot 68 D 451, SG (CI) Records, Box 2.

${ }_{296}$ Komer Memo, 21.5.63, ,Iran, 1/63[...] Memoranda', NSF, RWK, Box 424, JFKL.

${ }^{297}$ Vgl. Komer Memo, 20.6.63, ,Iran‘, Box 473, Harriman Papers, LOC.
} 
$\mathrm{zu}$ wichtigen Akteuren. Auf die eigentliche Regierungsebene und die wichtigsten Ministerien konnten US-Offizielle kaum spürbare Wirkung ausüben, zumal mit der Streichung der direkten Budgetbezuschussung ein weiteres, in der Vergangenheit wichtiges Mittel nun ebenfalls fehlte. Komer selbst begründete sein fortgesetztes Engagement mit der Notwendigkeit, die Errungenschaften der Weißen Revolution zu „konsolidieren“. Zu diesem Zweck, so Komer, seien auch weiterhin Interventionen gerade im personellen Bereich notwendig, zum Beispiel um die Ablösung des aus seiner Sicht schädlich handelnden Finanzministers Behnijā durchzusetzen. ${ }^{298}$

Abgesehen von der Frage der Form amerikanischer Unterstützung für die Weiße Revolution bzw. des Ausmaßes an wünschenswerter Intervention in die inneren Angelegenheiten und Entscheidungsprozesse Irans herrschte in den Sachfragen weiterhin Einigkeit. Die Botschaft bestätigte in ihrer Reaktion auf die Sitzung der NSC Standing Group den eingeschlagenen Weg und skizzierte ein sehr moderates Action Program in Reaktion auf NSAM N²28, inklusive der bereits etablierten Vorschläge für eine bedingungslose Unterstützung des Schahs, materielle und technische Hilfen für die Landreform, und die Förderung des 3. Entwicklungsplans. Geboten sei nun, den eigenen Einfluss auszuüben, ,largely through persuasion accompanied by sympathy and an occasional carrot". Die Botschaft betonte die Eigenständigkeit der iranischen Reformschritte und die nun allgegenwärtige Entschlossenheit „to help themselves“, ein indirekter Hinweis auf die nun nicht mehr gegebene Akzeptanz amerikanischer Intervention in zentralen politischen und ökonomischen Fragen und den amerikanischen Einflussverlust:

If we are expected to influence this Government, we cannot do it in an atmosphere where aid is judged on strictly banker criteria. Either we treat assistance as designed to influence policy here, with a recognized element of subsidy in the terms, or else we treat Iran as a friend in good financial shape, where we will make loans based on the cost of money to the United States Government on an arm's length business basis. We cannot have it both ways. ${ }^{299}$

Komers Bemühungen, die Botschaft wieder im Sinne aktivistischer Interventionen zu instruieren, blieben weitgehend ohne Wirkung. Holmes verteidigte seine Linie in Washington erfolgreich. ${ }^{300}$ Tatsächlich waren Komers Bemühungen, wenigstens die Instrumente der ehemaligen Task Force-Politik, wenn schon nicht deren Inhalte, zu erhalten, nicht mehr als ein Rückzugsgefecht. Allzu taktisch erschien sein Beharren auf wirtschaftspolitische Kursänderung und Personalwechsel sowie seine fortwährenden Begründungen, es gehe ihm nur darum die jüngsten Erfolge in der Iranpolitik zu konsolidieren: „We've done well in Iran recently, but it's sheer folly to risk letting Iran's hopeful 'white revolution' fade away because of a deficit of energy on our part.“"301 Angesichts der weitgehend kritiklosen, in Teilen eher

\footnotetext{
${ }^{298}$ Vgl. ebd.

${ }^{299}$ Tehran \#A-723, 13.6.63, POL, CF 63.

${ }^{300}$ Vgl. Kaysen Memo, 25.6.63, ,Iran, 1/63-11/63 (Folder 1 of 2), NSF, RWK, Box 424, JFKL; Komer und Bundy hatten Harriman wohl aufgrund seiner Seniorität als "hachetman \{sic!; hatchet man; R.P. $\}$ on Iran policy“ ausgewählt; Komer Memo, 20.6.63, ,Iran‘, Box 473, W. Averell Harriman Papers, LOC.

${ }^{301}$ Komer Memo, 20.6.63, ,Iran, 1/63[...] Memoranda‘, NSF, RWK, Box 424, JFKL.
} 
enthusiastischen Akzeptanz der „Revolution von Oben“ durch die Kennedy-Administration konnte es Komer nicht gelingen, eine zweite „Iran Exercise“ zu initiieren. Die seitens des State Department ausgearbeiteten Entwürfe für ein Action Program in Iran boten ein weiteres Mal nur moderate Unterstützungsschritte für einzelne Reformmaßnahmen, vermieden dafür, wie zu erwarten, jeden Hinweis auf eine Rückkehr zu aktivistischer US-Politik oder irgendwelche Vorschläge bezüglich politischer Reformen. Stattdessen betonte man „Überzeugungsarbeit“ anstelle von Versuchen, das Reformprogramm von außen zu „mikro-managen“. ${ }^{302}$ Komers Reaktion konnte nicht überraschen: „,[...] all objectives and excuses and mighty little «action» for an action program“, so seine Einschätzung über den „lousy“ Entwurf. ${ }^{303}$ Komers Kritik versandete jedoch wirkungslos angesichts der fehlenden Bereitschaft des Weißen Hauses, das State Department weiter unter Druck zu setzen.

Der Unwillen, sich weiter intensiv in Iran zu engagieren, ist umso erstaunlicher, wenn man die im Sommer 1963 immer offenkundiger werdenden Gefahren in Betracht zieht, die im Gefolge der Umsetzung des monarchischen Reformprogramms zu Tage traten. Seit dem Referendum im Januar hatte Mohammad Rezā weiter an Selbstvertrauen gewonnen und fühlte sich zunehmend in der Lage, die Weiße Revolution selbstständig voranzutreiben. Ein entscheidender Schritt hin zur weiteren Personalisierung des Reformprogramms war die Entlassung von Landwirtschaftsminister Arsanğāni im März 1963. Hauptsächlicher Grund für diesen Schritt war wohl die Argwohn des Schahs über die wachsende Popularität Arsanğānis auf dem Land, wo er - durchaus zu Recht - als die treibende Kraft hinter der Landreform angesehen wurde. Für die anstehenden Parlamentswahlen war zudem die Kontrolle der Wähler auf dem Land wie immer zentral, wohl ein weiterer Grund für den erzwungenen Rücktritt des Ministers. Mit Arsanğānis Abtritt war die erste „wilde“ Phase der Landreform abgeschlossen. ${ }^{304}$ Bereits vor seiner Entlassung war die Landreform etwas ins Stocken geraten. Bis Ende Mai 1963 waren weniger als die Hälfte der Großgrundbesitzern gehörenden Flächen an die Bauern verteilt worden - die Ausdehnung der Reform auf kleinere Flächen, wie noch im Januar beschlossen, wurde vorerst ausgesetzt. Zudem wurde nun auf die zuvor charakteristische sozialrevolutionäre Rhetorik verzichtet. ${ }^{305}$ Andere Teile des Reformprogramms wurden jedoch weiter vorangetrieben. Im Bewusstsein seiner nun noch

\footnotetext{
${ }^{302}$ Vgl. Report to the Standing Group, o.D., ,Iran, 1/63[... 2)‘, NSF, RWK, Box 424, JFKL; der Inhalt des Berichts folgte weitgehend, in Teilen wörtlich, dem oben genannten Airgram \#A-723 der Botschaft. Siehe Anm. 299.

${ }^{303}$ Komer Memo, 20.6.63; ein stark gekürzter zweiter Entwurf des State Departments für ein Action Program verzichtete gänzlich auf politische Stellungnahmen oder die Interventionsfrage und schilderte nur detailliert mögliche konkrete Hilfsmaßnahmen. Vgl. Action Program: Iran, Second Draft, 28.6.63, ,Iran, 1/63[...] 2)‘, NSF, RWK, Box 424, JFKL.

${ }^{304}$ Vgl. Tehran \#758, 14.3.63, , Iran General 3/63', NSF, Countries, Box 116A, JFKL; ein Hauptgrund war die Ernennung Mehdi Pirāstas zum neuen Innenminister - dieser würde über die Gendarmerie die anstehenden Wahlen kontrollieren. Die Botschaft glaubte an eine mögliche Rückkehr Arsanğānis, falls ,further brutal pushes" für das Programm notwendig werden sollten. Vgl. Tehran \#766, 14.3.63, POL 15-1 IRAN, CF 63.

${ }^{305}$ Vgl. Report to the Standing Group, o.D., ,Iran, 1/63 [... 2) $)^{6}$, NSF, RWK, Box 424; die Botschaft schätzte, dass die Verteilung der großen Ländereien den Zeitraum bis etwa Juni 1964, die darauf folgende Verteilung der kleineren Ländereien weitere 2-3 Jahre in Anspruch nehmen würde. In der ersten Stufe der Landreform waren etwa 10.-15.000 Dörfer betroffen. Bis Anfang Mai hatte der Staat ca. 4.400 Dörfer erworben. Das Land von ca. 2.400 Dörfern war an 140.000 Bauernfamilien verteilt worden. Vgl. Tehran \#A-518, 18.2.63, E 12 IRAN; Tehran \#A-696, 27.5.63, E 17 IRAN, CF 63.
} 
exponierteren öffentlichen Stellung untersagte Mohammad Rezā seiner Familie jedwede Einmischung in politische und finanzielle Fragen. ${ }^{306} \mathrm{Im}$ Anschluss an die noch symbolische Beteiligung von Frauen an der Referendumsabstimmung kündigte der Schah zudem die Einführung des aktiven und passiven Frauenwahlrechts für die kommenden Mağles-Wahlen an. ${ }^{307}$

Insbesondere die schiitische Hierarchie fühlte sich vom Frauenwahlrecht, aber auch von der Landreform herausgefordert, die die wirtschaftlichen Grundlagen ihrer gesellschaftlichen Stellung zu unterminieren drohte. Die anerkannte Führungspersönlichkeit - akzeptiert als einzige sogenannte „Quelle der Nachahmung“ (Marğa'-e Tağlid), der höchsten Stufe in der klerikalen Hierarchie - innerhalb der schiitischen Geistlichkeit, Ājatollāh Boruğerdi, bislang ein Vorreiter des quietistischen Verständnisses, hatte bereits das moderate Landreformgesetz Eg̉bāls scharf kritisiert, ein Vorzeichen der späteren direkten Konfrontation mit der Staatsmacht. $^{308}$ Der Widerstand gegen die Weiße Revolution vermengte sich zudem im Anschluss an den Tod Boruğerdis mit dem Wettbewerb um die Nachfolge als alleiniger Marğa', ein Rang, um den ab 1962 nicht weniger als acht Prätendenten konkurrierten. Es war vor allem der zuvor landesweit kaum in Erscheinung getretene Ājatollāh Ruhollah Musawi Khomeini (Xomejni) (1902-1989) - aufgrund seiner „Jugend“ von knapp 60 Jahren im Grunde chancenloser Kandidat angesichts der Seniorität der Konkurrenz -, der eine so noch nicht dagewesene Vermengung religiöser Autorität und aktueller politischer Fragen vollzog. ${ }^{309}$ Neben Landbesitzern und anderen klassisch konservativen Kreisen wie den Bāzāri sprach die religiös-oppositionelle Rhetorik auch nicht-säkulare Teile der nationalistischen Opposition wie die 1961 von Mehdi Bāzargān und anderen gegründete „Freiheitsbewegung Irans“ (Nehzat-e $\bar{A} z \bar{a} d i$-je Irān), eine Abspaltung von der Zweiten Nationalen Front, an. ${ }^{310}$ Im Gegensatz zu anderen führenden Klerikern gab sich Khomeini mit den begrenzten Konzessionen der 'AlamRegierung nicht zufrieden und begann, mit Unterstützung frommer Bāzāri, eine oppositionelle Organisation aufzubauen.

Noch im April 1963 hatte sich der Schah überzeugt gezeigt, dass er den Widerstand der

\footnotetext{
306 Vgl. Tehran \#A-505, 9.2.63, POL 15-1 IRAN, CF 63; dem Airgram zufolge hatte allein seine Zwillingsschwester Prinzessin Ašraf Schulden in Höhen von einer Million US-\$ angehäuft. Angeblich verweigerte der Schah von nun an, die Schulden der Familienmitglieder zu begleichen.

${ }^{307}$ Vgl. Tehran \#A-554, 6.3.63, POL 14 IRAN, CF 63.

308 Boruğerdis Protest übersetzt in FLOOR, Revolutionary Character, S. 504; zur Zusammenarbeit von Großgrundbesitzern mit der Geistlichkeit vgl. MAJD, Resistance to the Shah, S. 88-128; zur religiösen Opposition vgl. AKHAVI, Religion and Politics, S. 91-105.

${ }^{309}$ Vgl. MoIn, Khomeini, S. 69-81; MARTIn, Creating an Islamic State; ABrahamian, Khomeinism, S. 13-38; Hamid Algar, ,,Religious Forces in Twentieth Century Iran“, in: AVERY/HAMBLy/Melville (Hrsg.), Cambridge History of Iran, 7, S. 750-5; für die Hintergründe der klerikal-religiösen Konfrontation mit dem Pahlawi-Staat vgl. ARJOMAND, Turban for the Crown, S. 75-87; DABASHI, Theology of Discontent; für eine interessante Interpretation des theologischen Werdegangs Khomeinis und eine Identifikation der Ursprünge der späteren Theorie der „Statthalterschaft/Herrschaft der Rechtsgelehrten“, der ideologischen Grundlage des politischen Systems der späteren Islamischen Republik, in Khomeinis persönlicher Mystik vgl. MOAZAMI, Islamization; vgl. auch MAVANI Khomeini's Concept; MATIN, Making of Political Islam; ARJOMAND, Ideological Revolution.

${ }^{310}$ Zur Spaltung der Zweiten Nationalen Front vgl. KATOUZIAN, Struggle for Power, S. 228f.; vgl. JAHANBAKHSH, Religious Modernism in Iran, S. 91-112, CHEHABI, Liberation Movement, S. 156-60; TAGHAVI, Islamic Reformism in Iran, S. 59-111.
} 
schiitischen Geistlichkeit nun endgültig gebrochen habe. Zugleich habe er durch öffentliche Zurschaustellung seiner persönlichen Frömmigkeit Versuchen, die Führung als anti-religiös zu portraitieren, den Wind aus den Segeln genommen. Mohammad Rezā hoffte auf eine Isolierung „unerwünschter Elemente“ und die Schaffung einer aufgeklärten geistlichen Führung infolge des Konflikts, „[...] which would devote itself solely to religious matters.“311 Ähnliche Gelassenheit hinsichtlich der religiösen Opposition fand sich auch in Washington. Komer hatte bereits im April 1963 irrtümlich festgestellt, dass die bemerkenswerteste Tatsache im Zusammenhang mit der Weißen Revolution die ,comparative absence of effective opposition or violent response“ sei. Widerstand der Stämme und der Geistlichkeit, so Komer, seien nichts mehr als „,vestigial remnants of yesterday's business, not today's. “312

Tatsächlich organisierte sich der Widerstand schrittweise und fand in Khomeini einen charismatischen und zielstrebigen Organisator. Provokationen seitens der Regierung wie z.B. ein als Demütigung konzipierter Besuch des Monarchen im religiösen Zentrum Ġom im Januar, die Einberufung aller Theologiestudenten zum Militärdienst mit wenigen Ausnahmen führten schließlich Anfang Juni 1963 zum Ausbruch von Gewalt. ${ }^{313}$ Khomeini und die anderen religiösen Führer beabsichtigten, die Moharram-Riten und ihre besondere spirituelle wie emotionale Bedeutung für die schiitischen Gläubigen für neue Protestaktionen gegen die Regierung zu nutzen. Am 3. Juni 1963 hielt Khomeini in Ġom eine Rede, in welcher er den Schah öffentlich scharf attackierte. Das Referendum, so der Geistliche, sei ,anstößig “ gewesen, das Ergebnis erzwungen mit den Bajonetten der Sicherheitskräfte, und es widerspreche den Interessen der iranischen Nation. Zwei Tage später wurde Khomeini verhaftet - der Arrest wurde zum Aufbruchssignal für Großdemonstrationen in Ġom, Teheran und anderen Städten. Die Polizei wurde rasch überwältigt und erst durch den Einsatz der regulären Armee und die Anwendung massiver Gewalt gelang es, die Kontrolle wiederherzustellen. ${ }^{314}$ Die Botschaft bezeichnete die Ausschreitungen in Teheran und anderen Städten als die gewaltsamsten seit der Mosaddeg̈-Ära. In Teheran wurde das Kriegsrecht verhängt, es galt eine Ausgangssperre. Die Befürchtungen der Botschaft, Soldaten vom Land könnten sich weigern, während der AschuraRiten auf Geistliche zu schießen, bewahrheiteten sich jedoch nicht. Angesichts des Fanatismus einiger Oppositioneller befürchtete man zudem Anschläge auf das Leben des Schahs nach dem Muster der Fedā ijān-e Eslām in den 1950er Jahren.

\footnotetext{
311 Tehran \#892, 16.4.63, POL IRAN-US; der 27. Februar wurde zum wiederkehrenden Feiertag als „Tag der Frauenrechte“" erklärt. Vgl. Tehran \#A-636, 20.4.63, SOC 14-2 IRAN; tatsächlich gingen die Proteste der Geistlichkeit weiter, vor allem gegen das Frauenwahlrecht. Vgl. Meshed \#A-19, 22.3.63, POL 13-6 IRAN; Ausschreitungen in Aserbaidschan gegen das Frauenwahlrecht führten Ende März zu zwei Todesopfern. Vgl. Tabriz \#A-66, 27.3.63, SOC 14-2 IRAN, CF 63.

${ }^{312}$ Beirut \#924, 10.4.63, ,Iran General 4/1/63-4/19/63‘, NSF, Countries, Box 116A, JFKL \{Hervorheb. v. Verf.\}. ${ }^{313}$ Vgl. Tehran \#A-666, 14.5.63, POL 6-7 IRAN; Tehran \#A-77, 4.5.63, DEF 6-7 IRAN, CF 63; MoIN, Khomeini, S. $87 \mathrm{f}$.

${ }^{314}$ Zur Abfolge der Ereignisse während der Unruhen vgl. ebd., S. 102-112; diese Rede Khomeinis war zudem stark anti-israelisch geprägt. Siehe Khomeini Rede vom 3.6.63, in: AlgAR, Writings and Declarations, S. 177-80, hier 178 .
} 
Die schiitische Hierarchie ,[...] in essence launched a civil war against the regime“, so die Einschätzung NEAs im State Department. ${ }^{315}$ Zugleich glaubte man voraussagen zu können, dass - sollte sich das Schah-Regime halten können - ,[...] religion as an active political force in Iran will have been dealt a mortal wound. “316 Tatsächlich gelang die gewaltsame Niederschlagung der Proteste. Gerüchten zufolge musste der Schah allerdings erst von 'Alam von der Notwendigkeit überzeugt werden, die Tötung von Demonstranten in großer Zahl in Kauf zu nehmen. ${ }^{317}$ In Teheran demonstrierten nach Schätzungen 50.000 Menschen. Nach Ansicht "Alams waren darunter allerdings nur 2.000 religiöse „Fanatiker“ und der Rest der bei solchen Gelegenheiten immer anwesende Südteheraner Mob. Er sprach gegenüber dem Botschafter von 67 Getöteten allein in Teheran und 18 weiteren in anderen Städten. In der historischen Erinnerung war später von Tausenden von Toten im Juni 1963 die Rede, doch scheint dies der Aktenlage zufolge sehr unwahrscheinlich. Die Botschaft ging anfangs von etwa 125-200 Todesopfern aus, Opferzahlen von insgesamt etwa 300 bis 500 sind wohl realistisch - die in vielen Publikationen und in der offiziellen Erinnerung häufig zu findenden Opferzahlen von mehreren Tausend oder gar Zehntausenden Getöteter sind Ausdruck der späteren Revolutionsmythologie und deren unkritischer Übernahme in der Literatur. ${ }^{318}$

Nachdem wieder Ruhe in Teheran eingekehrt war, begann man mit der Analyse der Ereignisse. Die Botschaft sah den Widerstand einiger ,die hard Mullahs“ gegen das Reformprogramm des Schahs hinter den Unruhen, die Verhaftung Khomeinis als Auslöser derselben. Der im Vorfeld kaum bekannte Khomeini war nun aus Sicht der Botschaft die „bedeutendste politische Figur“, mit der sich die Regierung auseinanderzusetzen habe. Seine Popularität in konservativen Kreisen, insbesondere in den Basaren, sei enorm gestiegen und Gerüchten zufolge sei er nun ein ernsthafter Prätendent für die seit dem Tode von Ājatollāh Boruğerdi (1961) verwaiste Position der „Quelle der Nachahmung“ (Marğa'-e Tağlid), der höchsten Stufe in der schiitischen Hierarchie. Laut Botschaft werde von der breiten Bevölkerung in erster Linie die religiöse Opposition für die Intensität der Ausschreitungen verantwortlich gemacht. Letztlich

\footnotetext{
315 Talbot Memo, 6.6.63, FRUS 61-63, XVIII, S. 570.

${ }^{316}$ Ebd., S. 571.

${ }^{317}$ Der CIA Station Chief bestritt allerdings diese weit verbreitete Version der Zurückhaltung des Schahs: ,,So I think it's more a device to pin the blame on Alam, but Alam wouldn't have acted without the approval of his master."; Gratian Yatsevitch OHI, 5.11.88, OHIC; S. I-108; 'Alam selbst erinnerte sich Jahre später, er selbst habe die Niederschlagung der Proteste angeordnet und fast 90 Menschen hätten ihr Leben verloren. Siehe Eintrag vom 17./18.5.69, in: ALAM, Shah and I, S. 64f.

${ }^{318}$ Vgl. State \#1825, 14.6.63, POL 25-1 IRAN, CF 63; Tehran \#1076, 6.6.63, , Iran General 6/1/63-7/10/63‘, NSF, Countries, Box 116A JFKL; 'Alam brüstete sich in der Tat gegenüber Holmes mit seiner persönlichen Rolle bei der konsequenten Niederschlagung der Unruhen und verglich dies mit dem ,Throwing in the Sponge“ der ŠarifEmāmi-Regierung während der Unruhen vom Mai 1961; vgl. Tehran \#1150, 24.6.63, FRUS 61-63, XVIII, S. 601ff.; MoIN, Khomeini, S. 112, spricht basierend auf internen Polizeiberichten von 380 Toten; der iranische Menschenrechtsaktivist Emādaddin Bāgi berechnete auf Basis der Zahlen der Märtyrer- und Veteranenstiftung eine Gesamtzahl von 3.164 oppositionellen Todesopfern zwischen 1963 und dem Sieg der Revolution 1979, davon 32 während der Unruhen von 1963. Siehe http://www.emadbaghi.com/en/archives/000592.php\#more; selbst ehemalige amerikanische Diplomaten wie z.B. William Miller verbreiteten später die inkorrekte Angabe Tausender Todesopfer. Vgl. William G. Miller OHI, 7.3.85, IOHP, Transcript 2, S. 4; William Green Miller OHI, 10.2.2003, FAOH; ein Bericht des Konsulats in Isfahan bestätigte die „,extreme Popularität“" Khomeinis bei konservativen Muslimen. Während der Ausschreitungen seien vor allem Kinos und Spirituosen-Läden attackiert worden. Vgl. Isfahan \#A-56, 13.6.63, POL 25 IRAN, CF 63.
} 
könne diese ohnehin nur auf begrenzte Unterstützung in der Bevölkerung rechnen. Der Rückgriff auf die Sicherheitskräfte und die Armee angesichts des Aufstands sei indes nur notwendig geworden, da sich Iran gegenwärtig in einer Übergangsphase befinde. Die neue soziale Machtbasis der Bauern, drei Viertel der Gesamtbevölkerung, sei noch nicht politisch organisiert - sollte dies einmal der Fall sein, werde dieser neue veritable Machtfaktor auf Seiten der Monarchie wirksam werden, so das Wunschdenken. Die religiöse Opposition sei zwar „far from finished“, doch verfüge die Regierung über entsprechende Mittel, die Kontrolle zu bewahren. Ein Zusammengehen der überwiegend säkularen Nationalen Front mit der Geistlichkeit sei unwahrscheinlich und aufgrund der Schwäche der ersteren ohnehin kaum eine Bedrohung. ${ }^{319}$ INR im State Department gab sich dagegen skeptischer und zudem besorgt über die langfristigen Folgen der Zusammenstöße. Zum einen bestätigte die Analyse von INR den fortbestehenden Einfluss der Geistlichkeit:

Despite the persistent anti-clericalism of the Iranian Government during the past forty years, despite the general contempt in which educated Iranians hold mullahs, despite the fact that many uneducated Iranians consider mullahs to be parasites, there remains a deep stratum of religious convictions, sensibility, and emotionalism (not unmixed with xenophobia) which can be aroused by proper stimulation. ${ }^{320}$

Zwar fände das Vorgehen gegen die reaktionäre Rechte aus Geistlichkeit und Großgrundbesitzern durchaus Unterstützung bei gebildeten städtischen Schichten, doch ließe sich dieses nicht wirklich politisch verwerten. Zudem zeige sich gerade angesichts der angekündigten Mağles-Wahlen die Schwierigkeit, die befreiten Bauern oder auch die emanzipierten Frauen in schlagkräftige politische Kräfte zugunsten der Monarchie zu organisieren. Mit der gewaltsamen Niederschlagung der religiösen Opposition, der wirtschaftlichen Entmachtung derselben und der traditionell mit ihr verbündeten Schicht der Landbesitzer, und der Zerschlagung der nationalistischen Opposition in den Städten sei der Schah ,wahrscheinlich zur Zeit so nah an einer Monopolisierung politischer Macht wie noch nie in seiner Regentschaft“ “ ${ }^{321}$ Dennoch erwartete INR, dass Mohammad Rezā weiter auf die „klassische“ Methode der Machtausübung - ,persuasion, accommodation, compromise, intimidation, manipulation,, and intrigue as far as possible in preference to compulsion and dictation“ - vertrauen werde. Mit einer königlichen Diktatur basierend auf die militärische Gewalt sei nicht zu rechnen. Das Ausmaß der Gewalt sei allerdings ein „Rückschlag“ für das Schah-Regime nach innen wie außen, was sich vermutlich in einer weiteren Verlangsamung des Reformtempos niederschlagen werde. Positiv sei allerdings die zur Schau gestellte

319 Vgl. Tehran \#1098, 11.6.63, ,Iran General 6/1/63-7/10/63‘, NSF, Countries, Box 116A JFKL; spätere Untersuchungen der sozialen Zusammensetzung der Protestierenden zeigten, dass über die Hälfte der Beteiligten dem Kleinbürgertum zuzurechnen waren. Bauern machten nur etwa 4\% der Protestierenden aus. Vgl. MOADDEL, Ulama and the State, S. $542 \mathrm{ff}$.

${ }^{320}$ Denney Jr. Memo [RNA-30], 26.6.63, ,Iran, 1/63-11/63 (Folder 1 of 2), NSF, RWK, Box 424, JFKL; der Schah bemühte sich anschließend um eine Deeskalation im Verhältnis zur Geistlichkeit. Vgl. Tehran \#A-39, 18.7.63, POL 13-6, CF 63; zur komplexen Beziehung zwischen Basar und Geistlichkeit vgl. KESHAVARZIAN, Bazaar and State, S. 228-69.

${ }^{321}$ Siehe RNA-30, vorherig. Anm. 
Effizienz und Loyalität der Sicherheitsorgane. ${ }^{322}$

Tatsächlich kennzeichnet ein gewisser Unwillen die inneramerikanische Debatte, politische und soziale Ursachen der Unruhen genauer zu analysieren. Zwar hatte man zuvor die Lage in Iran als weitgehend stabil und die rechte Opposition als nicht fähig zu organisiertem und zudem gewaltsamem Widerstand eingeschätzt - dennoch einigte man sich rasch in der Bewertung der Ereignisse als wohl nicht zu vermeidende Reaktion auf die gewaltigen sozialen Umbrüche infolge der Weißen Revolution. Die Debatten im Anschluss drehten sich in erster Linie um die Leistung der verschiedenen, in der Regel durch die USA ausgebildeten Sicherheitsorgane während der Krise und die aktuelle Stabilität des Schahregimes. Eine alarmistische Einschätzung von militärischer Seite traf auf raschen Widerspruch seitens des State Department und der CIA ${ }^{323}$ Die Regierung, so konstatierte Holmes, habe bestimmt und besonnen reagiert and ,[...] has used force where it was required and, apparently, to the extent it was required.“ ${ }^{324}$ Sich distanzierend von der scharfen Kritik vonseiten des US-Militärs, verteidigte die CIA die rasche Überwältigung der städtischen Polizei durch die Demonstranten am ersten Tag der Unruhen angesichts des Missverhältnisses zwischen nur 500 Polizisten und Tausenden von Demonstranten. Die zur Verstärkung in die Stadt verlegten Truppen in Stärke von 13.000 Mann dagegen ,[...] performed well and showed no hesitancy in firing on the mobs when necessary. “325 Die Public Safety Division in Teheran verteidigte die Reaktion der Polizei als ,achtbar“. ${ }^{326}$ Auch in den Beratungen in der Special Group (CI) zeigte man sich zufrieden mit den iranischen Sicherheitskräften - letztlich habe man nun eine Demonstration der häufig in Frage gestellten Zuverlässigkeit der Armee erhalten, eine Einschätzung die der Schah in einem privaten Gespräch teilte: ,,a good lesson has been learned by all“‘327

\footnotetext{
${ }^{322}$ Ebd; zur „Islamisierung“ der iranischen Opposition vgl. MOAZAMI, Islamization; eine Einschätzung der Botschaft und ARMISH/MAAG vom August 1963 versicherte die grundsätzliche Loyalität der Streitkräfte zum Regime. Seit der Mosaddeg-Zeit seien die Streitkräfte „deeply penetrated and controlled“ durch die Sicherheitsorgane. Zudem seien die Luftwaffe, die Imperial Guards Division und die Special Forces unzweifelhaft loyal zum Schah und bereit, das Regime jederzeit zu verteidigen. Tehran \#A-128, 27.8.63, DEF 6 IRAN, CF 63. ${ }^{323}$ Die Defense Intelligence Agency gemeinsam mit dem Commander in Chief, U.S. Naval Forces, Eastern Atlantic and Mediterranean und dem U.S. Commander in Chief, Europe hatten ,kritische“ 3-4 Jahre für den Schah vorhergesagt, sollte ihm nicht der Aufbau einer neuen sozialen Unterstützungsbasis gelingen. Offenbar hatten die großen Schwierigkeiten der iranischen Armee in der Bekämpfung der Stammesaufstände in Fārs Zweifel an den Fähigkeiten der Sicherheitsorgane ausgelöst. Vgl. Krulak Memo, 13.6.63, FRUS 61-63, XVIII, S. 583-6; die CIA hatte bereits zuvor einen umfassenden und gemeinsamen Aufstand sämtlicher Minderheiten, insbesondere der etwa eine Million Kurden unter den 25 Millionen Iranern, als ,ominous security problem“ bezeichnet, hielt einen solchen aber für unwahrscheinlich. Anders als das US-Militär sah die CIA die Regierung im Juni 1963 im Konflikt mit den Stämmen im Süden infolge der gerade erfolgten Ermordung einer Reihe von Stammesführern als siegreich an. Vgl. CIA, Office of Current Intelligence Special Report, 17.5.63, ,Iran General 5/63،, NSF, Countries, Box 116A; CIA Report, Office of Current Intelligence, 13.6.63, ,Iran, 1/63-11/63 (Folder 1 of 2), NSF, RWK, Box 424, JFKL; Holmes beschwerte sich umgehend über die Einmischung des US-Militärs und kritisierte die militärischen Lageeinschätzungen als inakkurat und uninformiert: ,,it seems to me unreasonable to expect EUCOM \{das European Command; R.P.\} staff officers, working in Paris 3000 miles away, to produce accurate and reasoned over-all assessments of the complex and changing situation which prevails in Iran."; Tehran \#56, 19.7.63, POL 23 IRAN, CF 63.

${ }^{324}$ Tehran \#1098, 11.6.63, ,Iran General 6/1/63-7/10/63‘, NSF, Countries, Box 116A, JFKL.

${ }^{325}$ CIA Report, Office of Current Intelligence, 13.6.63, ,Iran, 1/63[...] 2), NSF, RWK, Box 424, JFKL.

${ }^{326}$ Vgl. Tehran \#A-44, 18.7.63, POL, CF 63, RG 59, NACP; in demselben Airgram distanzierte sich die Botschaft etwas von dieser Einschätzung und identifizierte das Eintreffen der Special Forces der Armee als Wendepunkt.

${ }^{227}$ Tehran \#1150, 24.6.63, FRUS 61-63, XVIII, S. 601; eine in der Kennedy Library verfügbare Version ohne Schwärzungen gibt zu erkennen, dass es sich um ein Gespräch des Schahs mit einem CAS handelt, also einer Controlled American Source, in diesem Fall wohl CIA Station Chief Yatsevitch. Das Dokument befindet sich in
} 
Tatsächlich bekam die eher zweifelhafte Behauptung der iranischen Behörden, die Unruhen seien von Nassers VAR finanziert und organisiert worden, mehr Aufmerksamkeit als deren eigentlichen Hintergründe. ${ }^{328}$ Ignoriert wurde z.B. die Tatsache, dass, anders als während früherer vorwiegend nationalistisch-säkularer Demonstrationen, in den jüngsten Unruhen aufgrund der xenophoben Stimmungen nun auch Amerikaner in Iran bedroht worden waren. Die sichtbare anti-israelische Symbolik in den Demonstrationen wurde letztlich auf die reaktionäre Weltsicht der „Mullahs“ zurückgeführt. Nur allzu gerne schenkte man den Beteuerungen des Schahs und 'Alams Glauben, jeder organisierte Widerstand sei nun gebrochen und man würde als nächsten Schritt die Eigenständigkeit der Geistlichkeit durch staatliche Reorganisation der religiösen Sphäre - unter anderem durch Schaffung eines Ministeriums für religiöse Angelegenheiten, wohl wieder nach kemalistischem Vorbild beenden. Der Widerstand gegen eine solche Maßnahme werde, so der Schah selbstbewusst, „ziemlich einfach zu brechen sein“. 329

Die Konsequenz der Entschlossenheit des Schahs, sein Reformprogramm energisch voranzutreiben, für die amerikanische Politik war, wenig überraschend, bedingungslose Unterstützung Mohammad Rezās. Im Juli 1963 teilte Präsident Kennedy dem Schah in einem Brief sein Mitgefühl über den Gewaltausbruch mit und versicherte fortgesetzte amerikanische

,Iran General 6/1/63-7/10/63‘, NSF, Countries, Box 116A, JFKL; vgl. Meeting of the Special Group (CI), 28.6.63, FRUS 61-63, XVIII, S. 607f.; angesichts der Unruhen stimmte nun das Pentagon der bereits seit Längerem gewünschten Entsendung von fünf zusätzlichen Experten für „riot control“ an die amerikanische Gendarmeriemission zu.

${ }^{328}$ Der Botschaft zufolge hatten der SAWAK-Direktor und auch der Schah eine ägyptische Beteiligung behauptet. Allerdings verfügte man über keine eigenständigen Informationen über Kontakte zwischen ägyptischen Agenten und schiitischen Geistlichen (im Gegensatz zu Kontakten mit der Nationalen Front und den Gašğā $i$ ). Überzeugendstes Indiz für ägyptische Beteiligung aus US-Sicht waren anti-israelische Slogans und Plakate, die vielleicht auf eine „Arab-inspired“ Kampagne verwiesen: „There has never been strong anti-Israel sentiment in Iran."; andererseits spricht einiges für israelische Versuche, den Verdacht auf die VAR zu lenken. Vgl. State \#A6, 18.7.63, POL, CF 63; TelAviv \#1025, 12.6.63, POL 24 IRAN-UAR, CF 63; die CIA bestätigte allerdings zu einem frühen Zeitpunkt eine mögliche Finanzierung auch von ägyptischer Seite. Vgl. CIA Report, 13.6.63, ,Iran, 1/63-11/63 (Folder 1 of 2), NSF, RWK, Box 424, JFKL; INR hingegen gab sich weitaus skeptischer bezüglich der vorgelegten Beweise für ägyptische Involvierung.

${ }^{329}$ Tehran \#1150, 24.6.63, FRUS 61-63, XVIII, S. 602; Tehran \#20, 6.7.63, ,6/1/63-7/10/63“, NSF, Countries, Box 116A, JFKL; der Schah unternahm im Anschluss Anstrengungen, die regierungsfeindlichen Geistlichen innerhalb der Hierarchie zu isolieren. Vgl. Tehran \#A-39, 18.7.63, POL 13-6 IRAN, CF 63; Überlegungen, mehr über die schiitische Geistlichkeit oder ihre Haltungen in Erfahrung zu bringen, wurden offenbar nicht angestellt. Selbst das immer aufgeschlossene INR im State Department sprach von „backward and obscurantist religious leaders“. Vgl. Denney Jr. Memo [RNA-30], 26.6.63, ,Iran, 1/63-11/63 (Folder 1 of 2), NSF, RWK, Box 424, JFKL; der Versuch einer Kontaktaufnahme durch schiitische Geistliche aus dem irakischen Nadschaf und eine öffentliche Aufforderung von Ājatollāh Šari`atmadāri zum Gespräch wurden auf Geheiß des Weißen Hauses ignoriert. Siehe Read Memo, 19.8.63, POL 25 IRAN; Meshed \#A-31, 18.6.63, POL 12 IRAN; bereits im Februar hatte es einen Bombenanschlag auf das Gebäude der Iran-America Society in Isfahan gegeben. Vgl. Isfahan \#A-35, 2.3.63, POL 25 IRAN, CF 63; Berichte des britischen Guardian im Juni 2016 über Kontakte zwischen Khomeini und den USA vor der Revolution erregten gerade in Iran einiges Aufsehen. Ein Rückblick der CIA aus dem Jahr 1980 erwähnte dabei offenbar einen Kontaktversuch Khomeinis im Jahr 1963, demzufolge der Geistliche die Botschaft rückversichert habe, er werde sich nicht gegen US-Interessen stellen und sehe vielmehr die US-Präsenz als wichtiges Gegengewicht gegen sowjetische und britische Einflussnahme. Siehe Saeed Kamali Dehghan/David Smith, „US had extensive contact with Ayatollah Khomeini before Iran revolution“, 10.6.2016, verfügbar auf https://www.theguardian.com/world/2016/jun/10/ayatollah-khomeini-jimmy-carter-administration-iran-

revolution; offenbar beruhte der CIA-Bericht auf einem vagen Gerücht, das die Botschaft im November 1963 nach Washington meldete, dem man aber kaum Glaubwürdigkeit beimaß. Vgl. Tehran \#A-74, 6.11.63; mein Dank für das Zusenden des Dokuments und für seine Einschätzung an den ehemaligen Assistant Secretary of State for Iran John Limbert im Februar 2018. 
Unterstützung : „I share the regret you must feel over the loss of life connected with the recent unfortunate attempts to block your reform programs. I am confident, however, that such manifestations will gradually disappear as your people realize the importance of the measures you are taking to establish social justice and equal opportunity for all Iranians. “330 Assistant Secretary Talbot interpretierte die Juni-Ereignisse als einen „Wendepunkt“ - geboten sei nun die Aufwertung der amerikanischen Unterstützung für die Weiße Revolution. ${ }^{331}$ Bezeichnend für die nun vollzogene und endgültige Kehrtwende der amerikanischen Iranpolitik weg von den Prinzipien reformistischer Intervention ist die Tatsache, dass der Ausbruch von Gewalt in Iran im Juni parallel zu den fortgesetzten Beratungen innerhalb der Administration über NSAM $\mathrm{N}^{\circ} 228$ stattfand, Auswirkungen auf dieselben allerdings kaum zu registrieren waren. In der verbliebenen Amtszeit der Kennedy-Administration schwelten die ungeklärten Fragen über exakte Zielsetzungen der US-Strategie gegenüber dem Reformprogramm und Ausmaß und Form der Einflussnahme zwar ungeklärt fort. Im Kern aber war mit der offiziellen Absegnung der Antwort des State Department auf die Anfrage des Weißen Hauses auch formell die Task Force-Politik, datierend vom Mai 1961, endgültig aufgehoben. Direkte Intervention in die Formulierung iranischer Innen- oder Außenpolitik mit dem Ziel, diese im Sinne eigener Vorstellungen $\mathrm{zu}$ verändern bzw. $\mathrm{zu}$ manipulieren, war nun kein akzeptiertes Mittel amerikanischer Iranpolitik mehr. Beratungen und Verhandlungen über zukünftige amerikanische Involvierung in Iran und US-Hilfsleistungen, insbesondere Militärhilfe, fanden nun noch stärker als zuvor auf bilateraler Ebene formell gleichgestellter, befreundeter und verbündeter Regierungen statt. Materielle und machtpolitische Asymmetrie zwischen den USA und Iran wirkten sich zwar auch weiterhin aus - allerdings verschwand der direkte amerikanische Einfluss auf die Innenpolitik Irans im Anschluss an das Amini-Experiment innerhalb weniger Jahre nahezu vollständig. Zugleich machte sich Mohammad Rezās Bestreben nach Unabhängigkeit und Selbstständigkeit im außenpolitischen Bereich und bald auch in der lange Zeit die gegenseitigen Beziehungen prägenden Militärpolitik zunehmend bemerkbar. Emanzipiert von der bisherigen Vormacht, entwickelte sich Iran schrittweise zu einem eigenständigen Akteur, dem es gelang, sich - vorwiegend in Gegensatz zu arabischen Nationalismus - in der nahöstlichen Region und in Umsetzung der sich bereits 1962 abzeichnenden Entspannung mit der Sowjetunion auch global neu zu positionieren. Zugleich schienen Weiße Revolution und monarchische Reform „Von Oben“ die Machtgrundlagen der Pahlawi-Dynastie innenpolitisch auf neue Säulen zu stellen. Der insbesondere 1961 befürchtete interne Zusammenbruch des Schah-Regimes blieb aus - tatsächlich erschien die Position Mohammad Rezās 1963 stärker als zu irgendeinem Zeitpunkt seiner Regentschaft. Wirtschaftliche Modernisierung wurde jedoch begleitet von immer schärfer werdender Repression und einer graduellen Ablösung des lange Zeit für Iran charakteristischen „milden Autoritarismus“. Aufgrund ihrer nun nahezu bedingungslosen Unterstützung für den Schah

\footnotetext{
${ }^{330}$ State \#32, 16.7.63, FRUS 61-63, XVIII, S. 646.

${ }^{331}$ Vgl. FRUS 61-63, XVIII, S. 571 n2.
} 
und die fortgesetzte exzessive Förderung des iranischen Militärs und anderer Sicherheitsorgane machten sich die USA in der Wahrnehmung breiter Teile der iranischen Bevölkerung zum Komplizen der Unterdrückung. Dieser längerfristige Effekt wurde allerdings erst offenbar mit der stark anti-amerikanisch akzentuierten iranischen Revolution von 1978/79.

Im Oktober 1963 versandte der Deputy Director (Intelligence) der CIA, Ray S. Cline, ein Memorandum an ausgewählte Offizielle der Administration, das er als „Homilie zu außenpolitischen Zielsetzungen“ titulierte. Das Memorandum fasste seine Überlegungen zu einer Grundproblematik amerikanischen außenpolitischen Handelns zusammen, den Wunsch nach „,besserer“ und gerechterer Regierungsführung in verbündeten Staaten und die häufige Realität von Repression und Missachtung der Menschrechte in diesen. Cline warnte vor Hoffnungen auf die Umsetzung von „Grand Designs“ - gemeint waren Versuche reformistischer Intervention - mit dem Ziel, humanere und gerechtere Ordnungen in diesen Ländern einzuführen. Sozioökonomische und politische Realitäten schränkten in der Realität amerikanische Einflussmöglichkeiten stark ein: „We must accept that our millions of dollars, our good intentions, and our political advice buy very little in the way of decisive influence.“ In der jüngeren Vergangenheit habe man zu viel gewollt - und dies zudem häufig öffentlich mit negativen Folgen für die eigenen strategischen Interessen. Es sei Zeit die ,politische und soziologische Augenwischerei“ zu beenden und die zentralen Interessen in den Mittelpunkt zu stellen:

[...] we want the governments of countries not committed against our existence (in other words, the non-Communist nations) at a minimum to preserve their political and strategic freedom of action from domination by any powerful, potentially hostile country. This is what we seek from 'neutral' nations. It ought always to be less well rewarded than our maximum requirement, which is a friendly government in a stable society. For nations which offer the latter, we should learn to work with their governing elites within the framework of their own value systems, which are often quite different from ours, in the direction of our compatible common interests. ${ }^{332}$ 